\title{
Scaphitoid cephalopods
}

of the

LIBRARY

BUREAO OF MINE

LIBRARY

SPUNANE. NASH

Colorado group

JUN 21971

PLEASE RETURTA

IO LIERARY

By W. A. COBBAN

GEOLOGICAL SURVEY PROFESSIONAL PAPER 239

Evolution of Scaphites and related genera, with

descriptions and illustrations of new species

and a new genus from $W$ estern

Interior United States

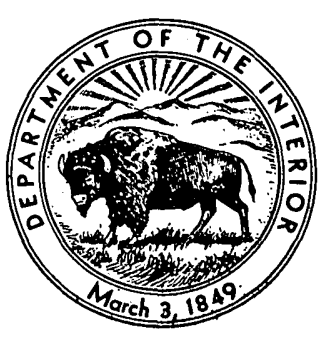

UNITED STATES GOVERNMENT PRINTING OFFICE, WASHINGTON : 1951 
UNITED STATES DEPARTMENT OF THE INTERIOR

Oscar L. Chapman, Secretary

GEOLOGICAL SURVEY

W. E. Wrather, Director

For sale by the Superintendent of Documents, U. S. Government Printing Office Washington $25, \mathrm{D}$. C. - Price $\$ 1.50$ (paper cover) 


\section{CONTENTS}

Abstract.

Introduction . . . . . Characteristics of the scaphites of the Colorado group.--

Scope of the group.

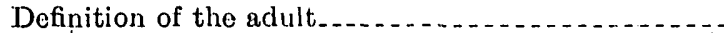

Size_._.

Form

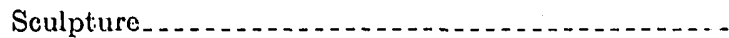

Suture...._...-.

Variation _. $\ldots \ldots \ldots \ldots \ldots \ldots \ldots$

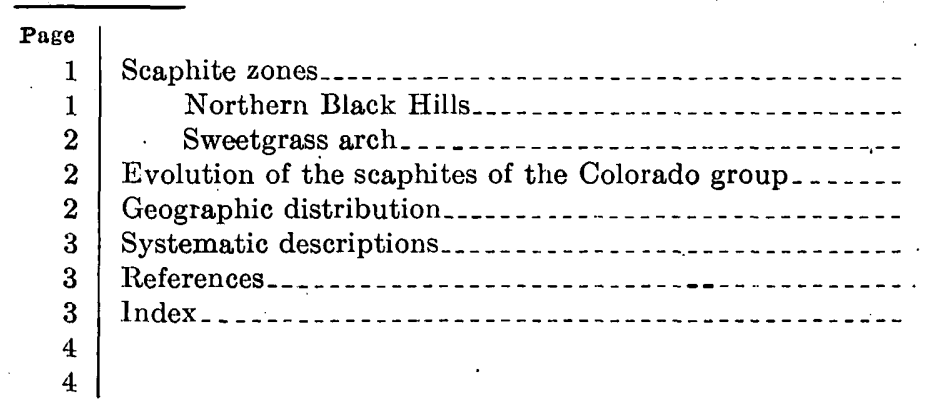

Page

4

4

5

6

11

18

39

\section{ILLUSTRATIONS}

Puates 1-21. Scaphites of the Colorado Group Page

Frgure 1. Lines of scaphite evolution

2. Sketches illustrating changes in size, form, sculpture, and suture of one lineage of scaphites of Niobrara age...- 8

3. Evolution of the trifid first lateral lobe.

4. Index map showing localities of collections from rocks of Colorado age

\section{INSERT}

Distribution of scaphitoid cephalopods of the Colorado group and equivalent rocks, by localities 


\title{
SGAPHITOID GEPHALOPODS OF THE COLORADO GROUP
}

\author{
By W. A. Cobban
}

\begin{abstract}
Scaphitoid ammonites are locally abundant in rocks of middle and late Colorado age in the Western Interior. These scaphites may be divided into two groups on the basis of evolute or involute septate coil in the adult. Evolute scaphites, which are known in the Western Interior only from the Greenhorn limestone, are not treated in this report. Involute scaphites are common throughout much of the Colorado rocks and fall within the genera Scaphites, Clioscaphites, and Desmoscaphites. Scaphites is represented by 39 species and varieties, of which 27 are described as new. The new genus Clioscaphites is represented by nine species and varieties, of which six are new. Desmoscaphites is known by only a single new species.

The adult scaphite is defined as an individual with partly unrolled living chamber, a distinctive sculpture, and a constricted aperture. The adults range in length from $7.7 \mathrm{~mm}$. to over 100 $\mathrm{mm}$. At most localities the smallest adult of a species is about one-half as long as the largest, but this ratio may be as much as $1: 3$ or even $1: 4$.

The scaphites have a considerable range in form. The adults of each species grade from small, slender individuals to large, stout ones. The large shell is more involute than the small one, and its living chamber is less extended. Several species have umbilical swellings, and one species has a lateral swelling at the base of the living chamber. The aperture, which is moderately constricted, has a small dorsal lappet. In addition, a few species have lateral and ventral lappets.
\end{abstract}

The sculpture consists of primary and secondary ribs, and, in many species, ventrolateral nodes. Generally, the primary ribs are strong and, at the ventrolateral margin, split into two or three weaker secondaries. One species has flat-crested and uncommonly high ribs that are curved back in cross section.

The external suture has four or five well-defined lobes and saddles that decrease in size away from the venter. The saddles are bifid, the first being asymmetric. The first lateral lobe is bifid in Scaphites, but asymmetrically bifid or trifid in Clioscaphites and Desmoscaphites. The internal suture consists of three or four lobes and saddles decreasing in size away from the dorsum.

The scaphites of the Colorado group fall into 15 faunal zones, 1 in the Greenhorn limestone, 7 in the Cartile shale, and 7 in rocks of Niobrara age. The most completely fossiliferous sections known are on the north flank of the Black Hills in South Dakota, and on the Sweetgrass arch of north-central Montana. The Black Hills section is best for the Greenhorn and Carlile formations, whereas the Sweetgrass arch is most fossiliferous for rocks of Niobrara age.

These species seem to have descended from some form like Scaphites aequalis Sowerby of the European Cenomanian. The earliest scaphites of the Colorado group, represented by $S$. delicatulus Warren, are part of a cosmopolitan fauna. Subsequent development in the Western Interior is distinctly provincial. Several lineages are indicated. The main line of scrphites shows, in the Greenhorn and lower part of the Carlile, trends toward increase in size, decrease in density of ribbing, and simplification of suture. At the beginning of middle Carlile time these scaphites suddenly decrease in size, and the maximum simplification of the suture is attained. The trend after that is toward increase in size of the adult and toward complexity in the suture. This line of scaphites reaches maximum size and complexity of suture by middle and late Niobrara time. By latest Niobrara time a definite trend toward decrease in size and simplification of suture is seen. By the beginning of Niobrara. time these scaphites show a tendency to become less unrolled, and by late Niobrara time, the dorsum of the adult living chamber is completely in contact with the outer septate coil. This tendency to become tightly enrolled is accompanied by a great reduction in the size of the umbilicus. The first lateral lobe of the suture is more or less symmetrically bifid in the Greenhorn, Carlile, and lower Niobrara species, but in the upper Niobrara species this lobe passes through an asymmetric phase and becomes trifid.

In the late Cariile a split from the main. scaphite line gave rise to a new line of species characterized by ventrolateral nodes. These scaphites closely pai allel the main line of species by becoming larger, less unrolled, and by developing a suture with trifid lobes.

A third lineage is represented by several tiny species. The oldest species, of earliest Carlile age, has a nearly normal aperture. Each younger species shows progressive forward extension of the lateral malgins of the aperture, and by early Niobrara time, prominent lateral lappets developed. By late Niobrara time the suture developed trifid lobes.

At the close of Colorado time the native Western Interior species were largely replaced by an entirely different type of scaphites which had migrated into the area from Europe by way of the Atlantic and Gulf Coasts of America.

\section{INTRODUCTION}

The scaphites are one of the most abundant and easily recognized groups of ammonites in the Upper Cretaceous of the Western Interior. They are especially abundant in marine rocks of Colorado age, and serve as the best guides for subdividing those beds into faunal zones. The scaphites are most abundant in dark-gray noncalcareous to somewhat calcareous shale, but their presence also in sandy and chalky beds makes them particularly useful in correlating diverse lithologic units. The earliest scaphites of Colorado age belong to an early Turonian cosmopolitan fauna, but their development thereafter is distinctly provincial. This makes it possible to study lineages without the disturbing influence of periodic migrations of foreign elements into the local populations. 
This study is based on a collection consisting of about 300 lots of fossils obtained largely by members of the United States Geological Survey from Montana, South Dakota, Wyoming, Utah, Colorado, Kansas, and New Mexico. Of the 3,300 specimens examined, about 1,500 were collected by the writer from measured stratigraphic sections.

The writer is deeply indebted to Dr. John B. Reeside, Jr., of the U. S. Geological Survey, whose broad experience in this field has been of great assistance and encouragement. Dr. Ralph W. Imlay and Dr. Roland W. Brown, of the U. S. Geological Survey, have offered much valuable advise. Thanks are also due Dr. Harold E. Vokes, of The Johns Hopkins University, for his guidance and many helpful suggestions in completing most of this work as part of a doctor's dissertation. Mr. Nelson W. Shupe, of the U. S. Geological Survey, photographed the fossils. .

\section{CHARACTERISTICS OF SCAPHITES OF THE COLORADO GROUP}

\section{SCOPE OF THE GROUP}

The genus Scaphites and closely related genera have been discussed fully by Reeside (1927a, pp. 5, 6; 1927b; $1927 \mathrm{c}$, pp. 20, 21). He summarizes the characters of the genus Scaphites as follows (1927a, pp. 5, 6):

* * * a normal coil of septate whorls and the last living chamber partly unrolled; whorls stout, umbilicus small, sculpture of straight ribs beginning in the umbilicus and passing with increasing height to the margin of the venter, where they split into two or more ventral ribs; there are also intercalated ventral ribs, and there may be definite nodes at the ventrolateral ends of the primary ribs; the suture consists of moderately incised elements, decreasing gradually in size from the median plane to the line of involution; lobes trifid in the earliest stages but usually bifid in the adult. Nowak called this group Holcoscaphites, but Parkinson's name [Scaphites] should be retained in a restricted sense.

The writer accepts this definition and is applying it in a broad sense. However, it is possible to subdivide the scaphites of the Colorado group into several groups of species as follows:

A. Adult septate coil subevolute to evolute. (Not treated in this work.)

B. Adult septate coil involute.

$a$. Small forms with living chamber moderately to considerably freed from the septate coil. Suture simple, symmetrically bifid first lateral lobe. Scaphites.

tetonensis

sagensis

frontierensis

uintensis

corvensis

nigricollensis

whitfieldi

ferronensis

warreni

veterinovus
carlilensis
morrowi
arcadiensis
larvaeformis
patulus
praecoquus
delicatulus

b. Large forms with living chamber moderately to slightly freed from the septate coil. Suture moderately complex, first lateral lobe either symmetrically or asymmetrically bifid. Scaphites.

binneyi.

interjectus

depressus

ventricosus

preventricosus

c. Large forms with living chamber not freed from the septate coil. Suture typically complex, first lateral lobe trifid or asymmetrically bifid. Clioscaphites.

novimexicanus

choteauensis

vermiformis

platygastrus

- montanensis

saxitonianus

d. Moderate sized forms with living chamber not freed from septate coil. Constrictions on early whorls. Suture typically complex, first lateral lobe trifid. Desmoscaphites.

erdmanni

e. Moderate sized forms with living chamber freed from septate coil. 'Ribs strongly recumbent and may have flat crests. Suture simple, symmetrically bifid first lateral lobe. Scaphites.

impendicostatus

mariasensis

$f$. Small forms with living chamber considerably fieed from septate coil. Aperture of adult with prominent lateral lappets. Suture simple, filst lateral lobe bifid or trifid. Scaphites.

coloradensis

auriculatus

\section{DEFINITION OF THE ADULT}

The most characteristic feature of the scaphites is that the last living chamber is partly unrolled. The sculpture on this last living chamber is entirely different from that of the septate whorls. It is this sculpture that readily distinguishes a species, whereas the sculptural features of the internal whorls of many species are almost identical. The last living chamber commonly has strong primary ribs that may end in ventrolateral tubercles, the ventral ribs may be uniformly spaced or widely spaced on one part and closely spaced on another, and the aperture is always constricted. Lateral lappets may be present. The adult scaphites are here defined as those individuals with partly unrolled living chamber bearing a distinctive sculpture and ending with a constricted aperture. Immature specimens that have the living chamber preserved do not differ 
in shape and sculpture from the internal septate whorls at comparable diameters of adult specimens.

\section{SIZE}

The scaphites of the Colorado group show a great range in size. The smallest known adult is a specimen of Scaphites coloradensis, $7.7 \mathrm{~mm}$. long, from rocks of late Niobrara'age. S. ventricosus, S. depressus, and Clioscaphites montanensis, of middle and late Niobrara age, attain lengths as great as $100 \mathrm{~mm}$. The average length of 550 adults from all the scaphite zones of the Colorado group is $39.2 \mathrm{~mm}$. (1.5 inches).

In any collection from any one locality a considerable range in size of the adults is noteworthy. The smallest individual is commonly about one half as long as the largest (pl. 18, figs. 7, 23, 24), and in some instances (compare pl. 18, fig. 23 with pl. 19, fig. 9), this ratio may be $1: 3$ or $1: 4$.

\section{FORM}

In all the scaphites of the Colorado group the umbilicus is wide in the first few whorls and narrow in the later ones. The umbilical shoulder rounds evenly into the narrow, steeply inclined umbilical wall and into the flattened or broadly rounded flanks. The whorl cross sections are normally wider than high, but the youngest whorls of the scaphites of late Carlile age may be as high as wide, or occasionally higher than wide (pl. 5, fig. 2). The venter of most species is well rounded to broadly rounded, but the last septate whorl of Clioscaphites vermiformis (Meek and Hayden) may have a flattened venter, and the new variety $C . v$. toolensis may even have a broadly depressed venter near the orad end of the septate coil. The venter rounds evenly into the flanks, and in many species it is difficult to determine where one passes into the other. Because of this difficulty and to maintain a uniform scheme of description, the point where the primary ribs fork is taken as the margin of the venter in most specimens. This is the point where the primaries commonly attain their greatest height or where tubercles may develop. Using this as a guide the scaphites have a much greater area in the venter than most other ammonites have.

On the third or fourth whorls succeeding the protoconch the ventrolateral margin is subangular to sharply rounded and, on many individuals, it is raised into a thickened ridge. On earlier and later whorls the ventrolateral margin is considerably more rounded.

The body chamber is large, making up one-half to three-fourths of a whorl. In Scaphites the adult living chamber is slightly to almost wholly freed from the septate coil. Where only slightly freed from the septate coil, it is the younger part of the living chamber that is not in contact with the coil. In Desmoscaphites and Clioscaphites, n. gen., the living chamber is wholly or nearly entirely in contact with the outer septate whorl. All specimens have a persistent dorsal furrow on the freed body chamber. The aperture is reniform in cross section and moderately constricted. A small, broad dorsal lappet is present in all adults. It is most conspicuous in Scaphites, especially in some of the species of Carlile age (pl. 5, fig. 25 ; pl. 6, fig. 5). Scaphites tetonensis and $S$. impendicostatus, $\mathrm{n}$. spp. have the ventral margin of the aperture bent away from the septate coil. In the latter species the shell is thickened along the ventral margin (pl. 11, fig. 8). Two tiny species of Niobrara age, $S$. auriculatus and $S$. coloradensis, n. spp., have dorsal, ventral, and lateral lappets; the last is narrow, pointed, and directed laterally.

An umbical swelling occurs at the base of the adult living chamber of $S$. delicatulus Warren and S. impendicostatus, and in a few specimens of S. larvaeformis Meek and Hayden, S. patulus, n. sp., Clioscaphites mon. tanensis, n. sp., and C. novimexicanus (Reeside). Many specimens of $S$. nigricollensis, $\mathrm{n}$. sp. have a lateral swelling at the base of the living chamber.

\section{SCULPTURE}

The sculpture consists of numerous primary (umbilical) and secondary (ventral) ribs, and in addition, many species have a row of ventrolateral tubercles which may be round or radially elongate. The primary ribs are inclined backward as they cross the umbilical wall, and on reaching the umbilical shoulder, bend forward and cross the flank with a forward inclination of $15^{\circ}$ to $45^{\circ}$. At the ventrolateral margin (about half way from the umbilical seam to the middle of the venter) the primaries attain their greatest height and then split into two or three weaker secondaries. These may (1) extend straight across the venter, as on the living chamber of Scaphites ventricosus Meek and Hayden (pl. 12), (2) cross the venter with a backward bending, as on the last septate whorl of $S$. patulus, n. sp. (pl. 1, fig. 27), (3) cross the venter with a forward arching, as on S. whitfeldi, n. sp. (pl. 4, fig. 37), or (4) curve back from the ventrolateral margin and then cross the middle of the venter with a forward arching, as on the immature stages of Clioscaphites vermiformis (Meek and Hayden) (pl. 18, figs. 12-18). Intercalated ribs are common between the paired secondaries and begin at the point of furcation or below it on the flanks. The ribs are either sharp or rounded, but one species, Scaphites impendicostatus, n. sp. (pl. 11), possesses flat-crested as well as unusually high ribs that are curved backward in cross section. Pointed, round tubercles are present in $S$. delicatulus; $S$. larvaeformis, S. patulus, S. praecoquus, n. sp., S. arcadiensis Moreman, S. uintensis, n. sp., S. frontierensis, n. sp., S. sagensis, n. sp., S. binneyi Reeside, Clioscaphites vermiformis, $C$. 
platygastrus, n. sp., C.? choteauensis, n. sp., and Desmoscaphites erdmanni, n. sp.

\section{SUTURE}

The suture ranges in complexity from the simple pseudoceratitic type characteristic of the middle Carlile species to the highly incised form of the late Niobrara species. The external suture has four or five clearly defined lobes and saddles progressively decreasing in height away from the venter. The saddles are bifid, and the first is asymmetric with the widest part on the ventral side. The first lateral saddle is always wider than the second, but the second may or may not be broader than the third. The first lateral lobe is bifid in Scaphites and trifid or asymmetrically bifid in Clioscaphites and Desmoscaphites. The second lateral lobe is typically bifid in the early Carlile species, but commonly trifid in those from the late Carlile and Niobrara. The rest of the external lobes are trifid, bifid, or undivided. The internal suture consists of three or four lobes and saddles decreasing in size away from the middle of the dorsum. The dorsal lobe is long, slender, and trifid. The first internal lobe is bifid or trifid and may be nearly as large as the dorsal lobe. The remaining lobes are small and commonly bifid. The internal saddles are small and bifid.

\section{VARIATION}

The scaphites are a highly varied group. The adults of each species grade from small, slender forms to large, stout forms. Because of the more slender whorls, the small adults are less involute and the living chamber is more freed from the septate coil (compare pl. 1, figs. $8,16 ; \mathrm{pl} .15$, figs. 1,7$)$. In many species the ribs tend to be denser and weaker on the living chambers of the larger adults (pl. 7, figs. 6, 12). In some species the large and small adults.differ considerably in shape and sculpture, and perhaps only the use of large collections that show the intergradations prevents mistaking the large and small forms for different species. For example, the internal molds of the living chambers of the large, stout form of Scaphites nigricollensis, $\mathrm{n}$. sp. tend to be smooth and commonly are inflated laterally near the base (pl. 6). In contrast the internal molds of the small, slender form are strongly ribbed and show no trace of lateral swellings (pl. 5, figs. 11-25). In dealing with such varied species, varietal names are useful.

\section{SCAPHITE ZONES}

The most completely fossiliferous sections containing scaphites are on the flanks of the Black Hills and on the Sweetgrass arch of north-central Montana. The Black Hills section includes nearly all the zones of the Greenhorn and Carlile formations, and the Colorado shale of the Sweetgrass arch demonstrates nearly all the zones of Niobrara age. Collections from other localities in the Western Interior indicate that the Black Hills and Sweetgrass arch sequences each lack a scaphite zone, which may indicate hiatuses or that the particular ages may be represented by poorly fossiliferous or unfossiliferous beds. Scaphites have not been found in the Western Interior in pre-Greenhorn strata.

\section{NORTHERN BLACK HILLS}

The Niobrara formation of the Black Hills contains few fossils other than Inoceramus fragments, Ostrea congesta Conrad, Foraminifera, and fish bones. Scaphites have not been found in the Niobrara formation but the Carlile shale contains a rich scaphite fauna, and the underlying Greenhorn formation has yielded some specimens.

The following generalized section shows the major lithologic units that compose the Greenhorn, Carlile, and Niobrara formations and the scaphite species found in each. This section was measured 4 to 9 miles north of Belle Fourche, S. Dak.

Niobrara formation (200 feet)

16. Chalk marl, weathers white; interbedded with many thin layers of bentonite................ Carlile shale (546 feet)

Sage Breaks member (194 feet)

15. Shale, dark-gray; weathers dark; contains abundant gray-weathering calcareous concretions that are septarian, with thick seams of brown, yellow, and white calcite. Sca-

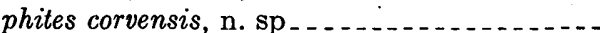

Turner sandy member (258 feet)

14. Shale, gray, finely sandy; weathers buff gray; contains tan- and yellow-weathering calcareous concretions. Scaphites corvensis, $\mathrm{n}$. sp., near top, S. nigricollensis, n. sp., in middle, and $S$. whitfieldi, n. sp., at base...-

13. Shale, dark-gray; weathers dark; contains some sandy beds and numerous rusty- and reddish-weathering ferruginous concretions. Scaphites whitfieldi, n. sp., and S. pisinnus, n. sp . . . . . . . . . . . . . . . . . . . . . . .

12. Shale, dark-gray; weathers medium gray; contains large yellow-weathering calcareous concretions and small gray-weathering calcareous concretions. Scaphites warreni Meek and Hayden in lower half . . . . . . . . . . . .

11. Shale, gray, very sandy; weathers mediumgray; contains large yellow-weathering sandy calcareous concretions at top and in middle; chert pebbles and coarse sandstone at base. Scaphites warreni Meek and Hayden and

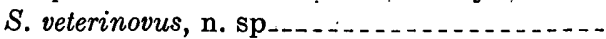

Unnamed shale member ( 94 feet)

10. Shale, dark-gray . ... . . . . .

9. Shale, dark-gray; weathers medium-gray; contains numerous ferruginous concretions weathering orange tan, rusty, and darkmaroon. Scaphites carlilensis Morrow and S. arcadiensis Moreman.................
Feet 200 
Carlile shale-Continued

Unnamed shale member-Continued

8. Shale, dark-gray, slightly sandy ...........

7. Shale, black-gray, hard . . . . ................

6. Shale, dark-gray, slightly calcareous, weathers bluish gray; contains light-gray limestone concretions at top. Scaphites larvaeformis Meek and Hayden, S. patuluis, n. sp., and $S$.

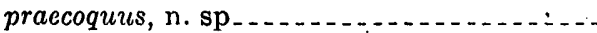

Greenhorn formation (312 feet)

5. Shale, gray, calcareous; weathers white; contains thin lenses and shaly layers of limestone. . . . . .

4. Shale, gray, calcareous; weathers white; contains white-weathering limestone concretions.

3. Shale, black-gray, noncalcareous; contains small yellow limonite nodules . . . . . . . . . . . . . . .

2. Shale, gray, calcareous; weathers gray; contains ferruginous concretions and ferruginous shaly. layers weathering rusty. Scaphites delicatulus

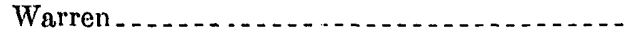

1. Shale, gray, slightly calcareous; weathers giay -..

In the Black Hills section the scaphites occur in the following sequence:

Carlile shale

Zone of Scaphites corvensis, n. sp.

Zone of Scaphites nigricollensis, n. sp.

Zone of Scaphites whitfieldi, n. sp.

Zone of Scaphites warreni Meek and Hayden

Zone of Scaphites carlilensis Morrow

Zone of Scaphites larvaeformis Meek and Hayden

Greenhorn formation

Zone of Scaphites delicatulus Warren

The beds from which the scaphites of the lower four \%ones of the Carlile shale were collected are separated by unfossiliferous shale. This may explain the fact that the ranges of the species do not overlap. In contrast, the Turner sandy member containing $S$. whitfieldi, S. nigricollensis, and $S$. corvensis is fossiliferous throughout and, apparently, there are no breaks in sedimentation. The result is that transitional forms occur between the species of each of these zones.

Collections from the top of the Frontier formation of the Laramie Basin of southeastern Wyoming and from the Mancos shale of central Utah and northwestern New Mexico contain a scaphite species.intermediate between S. warreni and S. whitfieldi that occurs between the zones of these species. This species, S.ferronensis, n. sp., may eventually be found in the upper part of unit 12 of the northern Black Hills section.

\section{SWEETGRASS ARCH}

The Colorado sbale of the Sweetgrass arch contains equivalents of the Colorado group of the Black Hills. The beds equivalent to the Greenhorn and Carlile formations are lithologically similar to those of the Black Hills but considerably thinner. In contrast the beds rect equivalent to the Niobrara formation are several times as thick and, unlike the Niobrara formation, are composed almost entirely of dark-gray noncalcareous shale. Scaphites have not been found in the Greenhorn equivalent, and only two zones can be recognized in the rocks of Carlile age. The overlying shale, however, contains the richest known scaphite faunas of Niobrara age.

The following generalized section shows the major lithologic units of that part of the Colorado shale equivalent to the Greenhorn, Carlile, and Niobrara formations of the Black Hills. The beds of Greenhorn and Carlile age were measured along Sun River valley west of Great Falls, and the Niobrara rocks were measured on the west and south flanks of the Kevin-Sunburst dome.

Colorado shale

Beds equivalent to the Niobrara formation (620 feet) Feet

12. Shale, dark-gray; contains g1ay-weathering calcareous concretions and about 10 thin layers of bentonite Clioscaphites novimexicanus (Reeside), Desmoscaphites erdmanni, n. sp., and Scaphites leei Reeside in upper part; Clioscaphites? choteauensis, n. sp., in middle part; Clioscaphites montanensis, $\mathrm{n}$. sp., C. vermiformis (Meek and Hayden), C. saxitonianus (McLearn), and Scaphites coloradensis, n. sp., in lower part..........

1. Shale, dark-gray; contains ferruginous concretions weathering rusty and reddish brown and calcareous concretions weathering gray, buff, and yellow. Very few bentonite beds. Scaphites ventricosus Meek and Hayden and S. tetonensis, n. sp. in lower half ..........

10. Shale, dark-gray, contains abundant grayweathering calcareous concretions and about 40 layers of bentonite. Scaphites preventricosus, n. sp., S. impendicostatus, n. sp., and $S$. auriculatus, n. sp., in upper two-thirds. S. mariasensis, n. sp., in lower third....... Beds equivalent to the Carlile shale (155 feet)

9. Shale, dark-glay, sandy, hard; weathers bluish gray; contains gray- to yellow-weathering calcareous concretions and rarely a chertpebble layer. Scaphites nigricollensis, n. sp.

8. Shale, dark-gray, finely sandy; contains numerous ferruginous concretions weathering rusty to reddish brown. Scaphites carlilensis Morrow

7. Shale, dark-gray, finely sandy, hard Beds equivalent to the Greenhorn limestone (55 feet)

6. Chalk marl, dark-gray; weathers buff; contains thin gray limestone lenses and thin

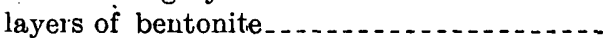

5. Shale, gray, calcareous.........

4. Chalk marl, dark-gray; weathers light blue...

3. Bentonite, creamy white .

2. Shale, brownish, soft, papery, noncalcareous.-

1. Chalk marl, gray; weathers buff; contains small ferruginous concretions and larger gray-weathering calcareous concretions...... 
The rarity and poor preservation of fossils in the beds of Carlile age may account for the recognition of only two of the six scaphite zones known in the Carlile shale of the Black Hills. However, Scaphites nigricollensis occurs so close above the beds containing $S$. carlilensis that probably the zones of $S$. warreni, $S$. ferronensis, and $S$. whitfieldi are absent or they are represented by very thin, unfossiliferous zones.

The shale of Niobrara age shows the following scaphite zones.

\section{Zone of Clioscaphites novimexicanus (Reeside) \\ Zone of Clioscaphites? choteauensis, n. sp. \\ Zone of Clioscaphites montanensis, $\mathbf{n}$. sp. \\ Zone of Scaphites ventricosus Meek and Hayden \\ Zone of Scaphites preventricosus, n. sp. \\ Zone of Scaphites mariasensis, n. sp.}

This is the most complete record known for beds of Niobrara age. However, collections from the Cody shale of the Bighorn Basin, Wyoming, reveal a species, Scaphites depressus Reeside, intermediate in form between S. ventricosus and Clioscaphites montanensis and occurring between those zones. On the Sweetgrass arch $S$. ventricosus and $C$. montanensis are found in such close stratigraphic proximity that probably the $S$. depressus zone is absent. Phosphatic nodule layers present in the shale just above the highest occurrence of $S$. ventricosus also suggest hiatuses in this part of the sequence.

\section{EVOLUTION OF THE SCAPHITES OF THE COLORADO GROUP}

Spath (1934, p. 498) has pointed out that the family Scaphitidae may have had its origin in the Ly toceratidae of the Mediterranean-Equatorial region, and that the earliest known scaphites occur in the upper Albian. These early scaphites are tiny and have either evolute or involute septate whorls in the adult stage. The sutures are moderately incised and consist of but few elements. The suture of Scaphites circularis Spath (1937, p. 501, text fig. 175d) from the English Gault has only six lobes and six saddles. These little scaphites are comparatively rare in the Albian, but in the Cenomanian they are more abundant, especially $S$. aequalis Sowerby. The sutures of the Cenomanian forms seem to be less incised than those of their Albian ancestors.

In America the oldest described scaphite is Worthoceras worthense (Adkins and Winton) (1920, p. 36, pl. 7, figs. $1,2)$, a slightly evolute form from the Duck Creek limestone of the basal upper Albian of Texas. Scaphites hilli Adkins and Winton (p. 37, pl. 7, figs. 3-6) of the Pawpaw formation of the uppermost Albian of Texas is an involute form. The lower Cenomanian Grayson shale of Texas has yielded a tiny involute scaphite, $S$. bosquensis Böse (1928, pl. 224, pl. 7, figs. 1-6), and a larger evolute form, S. subevolutus Böse (p. 225, pl. 7, figs: $7-30 ;$ pl. 18, fig. 8). Several small involute and evolute scaphites have been described from the Cenomanian of Oregon and California by Anderson (1902).

Scaphites have not been found in the Cenomanian Woodbine group of Texas (L. W. Stephenson, personal communication), or in the equivalent beds of the Western Interior.

The lower Turonian zone of Sciponoceras gracile (Shumard) marks the first widespread occurrence of scaphites in North America. Scaphites delicatulus Warren $(1930$, p. 66 , pl. 3, fig: 3 ; pl. 4, figs. 7,8$)$ is known from the Alaskan arctic slope, from basal Turonian rocks of the Mackenzie River valley of northwestern Canada, from the lower part of the Smoky River shale of the Grande Prairie district of north central Alberta, from the Greenhorn formation of the Black Hills, and from equivalent bed's in the Cody shale on the northeast flank of the Bighorn Mountains. S. brittonensis Moreman (1942, p. 215, pl. 34, figs. 1, 2; text fig. 2r), from the Eagle Ford group of Texas, very closely resembles $S$. delicatulus and is also associated with Sciponoceras gracile (Shumard). The resemblance of these scaphites to S. aequalis Sowerby of the European Cenomanian is striking. This close similarity, and the absence of scaphites in the post-Grayson Cenomanian rocks of the Gulf Coast and Western Interior, suggest that $S$. aequalis gave rise to $S$. delicatulus through some transitional form that migrated to America during the early Turonian.

Scaphites delicatulus var. greenhornensis, n. var., from the Greenhorn formation differs from the typical form of the species chiefly in possessing coarse ribbing on the curved part of the living chamber and in having proportionally more primary ribs and fewer secondaries on the living chamber. From this type of ancestor $S$. larvaeformis Meek and Hayden of the basal Carlile shale was probably derived. This species differs from $S$. $d$. greenhornensis in having fewer ribs and a smaller umbilical swelling. About one-half of the specimens of $S$. larvaeformis at hand have coarse ribbing on the last half of the living chamber although not as coarse as on the Greenhorn variety. S. praecoquus, n. sp., can be interpreted either as a split from $S$. larvaeformis or as a tiny variant that developed an uncoiled living chamber before other adult features were fully developed. It appears to mark the beginning of a line of small scaphites that by Niobrara time had developed prominent lateral lappets. S. patulus, n. sp., also of the basal Carlile, is either a split from $S$. larvaeformis or a descendent of $S$. delicatulus that developed a more curved and strongly sculptured living chamber considerably depressed in cross section. 


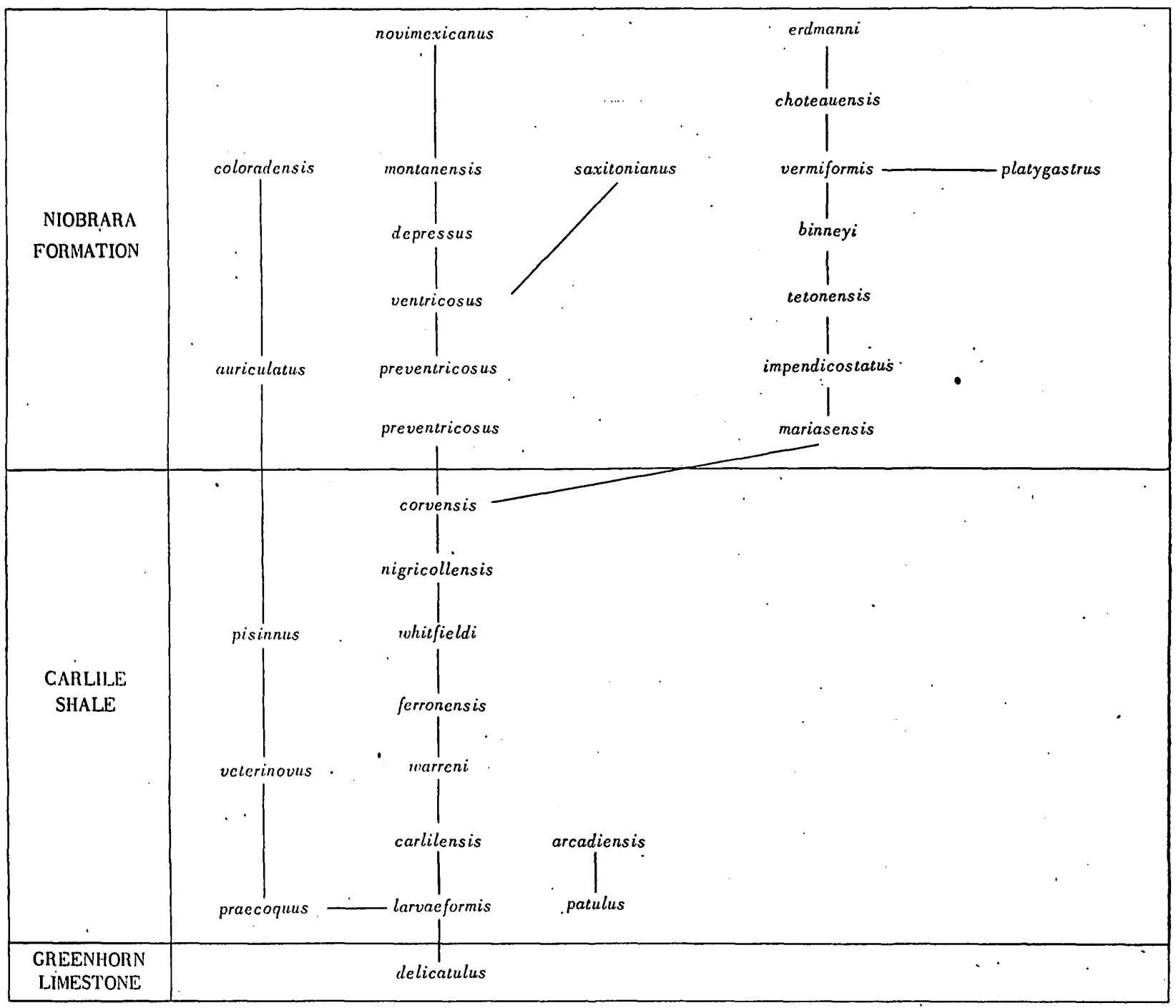

FIGURE 1.-Lines of scaphite evolution.

S. patulus and $S$. larvaeformis sbow backward bending of the ribs crossing the venter on the last septate whorl, a character found in many early scaphites throughout the world.

Scaphites carlilensis Morrow, which marks the zone next above that of $S$. larvaeformis, is most abundant in the Blue Hill shale member of the Carlile shale of Kansas, and in lithologically similar beds that form most of the unnamed shale member of the lower part of the Carlile shale of the northern Black Hills. S. carlilensis attains a larger size than $S$. larvaeformis and the suture is less incised. There is. further reduction in the number of ribs, and these are now uniformly spaced on the venter of the living chamber. Tubercles and umbilical swelling are lacking. The associated species, S. arcadiensis Moreman, still maintains ventrolateral tubercles and a depressed cross section of the living chamber, suggesting derivation from S. patulus. The tubercles, however, are much less conspicuous and tend to become high primary ribs. Both S. carlilensis and S. arcadiensis have lost the rursiradiate ribbing of the ventral ribs on the last septate whorl.

Scaphites warreni Meek and Hayden characterizes the lower third of the Turner sandy member of the Carlile shale of the Black Hills and equivalent sandy beds in eastern Wyoming, Colorado, and New Mexico. This species is smaller than its ancestor, S. carlilensis, possibly because of an unfavorable environment. The sediments containing $S$. warreni are sandy and, in central Utah, pass into cobble beds and variegated clays of nonmarine origin reflecting contemporary orogenic movements west of the region. The scaphite suture became progressively simpler after Cenomanian time and reached its simplest state attaining a.pseudoceratitic stage in this species (pl. 3 , figs. $8,20,22$ ). The trend for ribbing to become dense on the curved part 

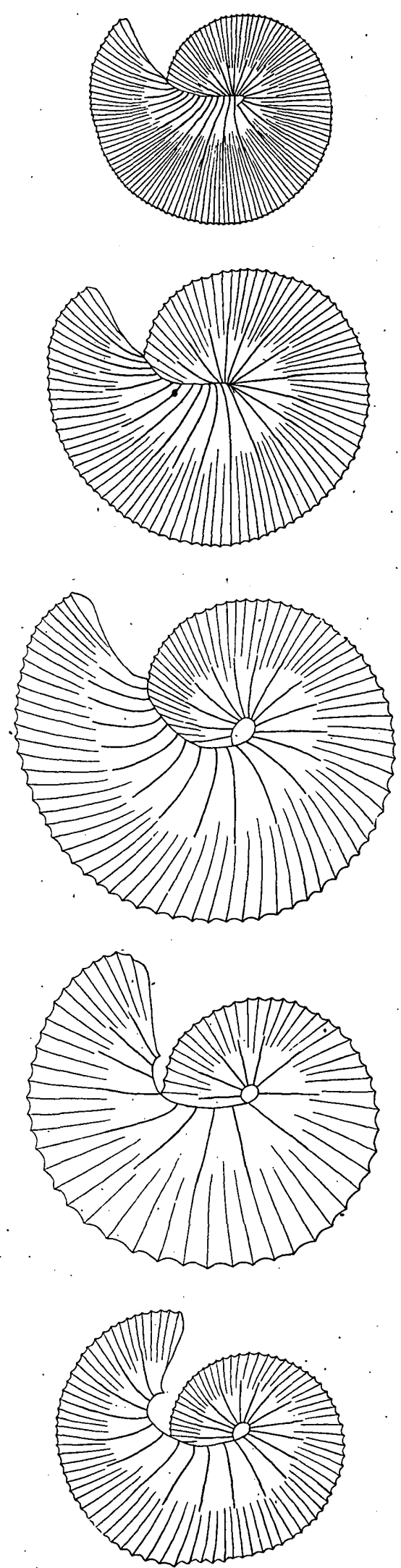

FIGURE 2.-Sketches illustrating changes in size, form, sculpture, and suture of one lineage of scaphites of Niobrara age.
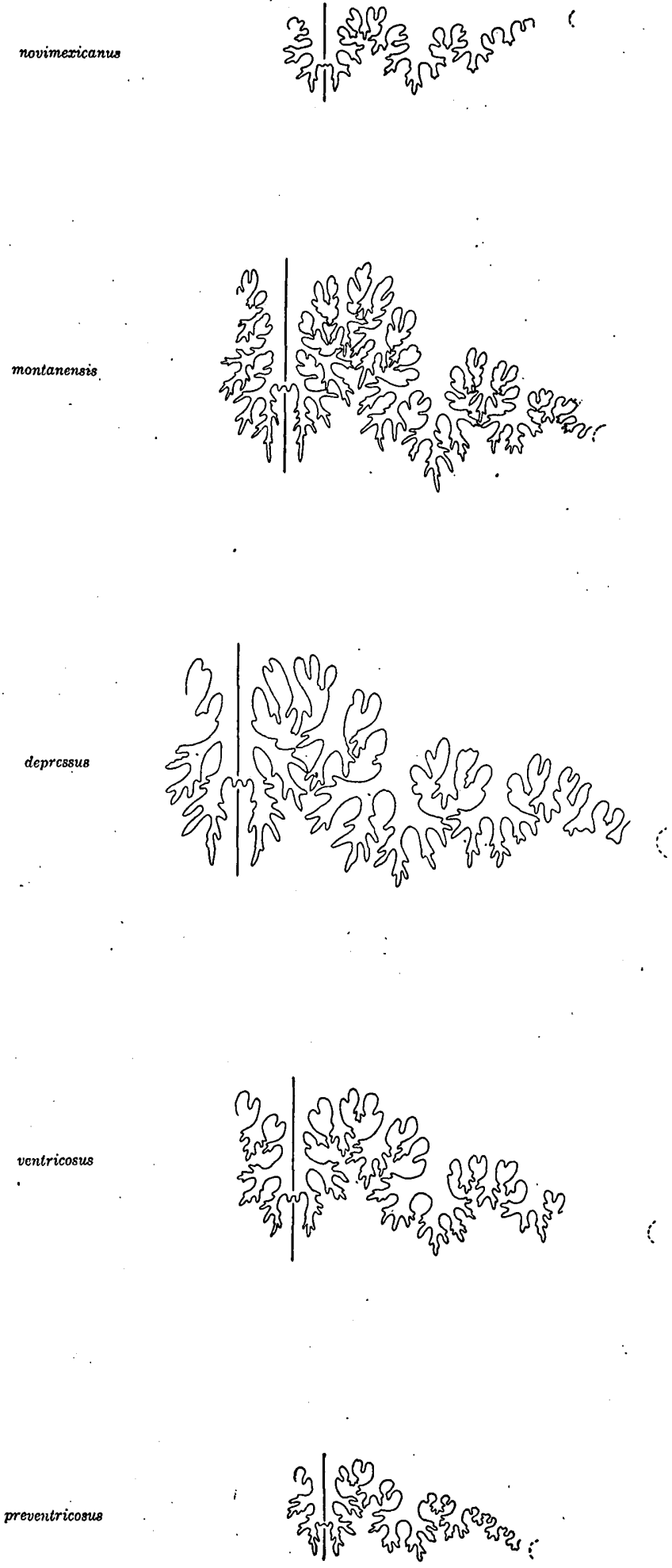
of the living chamber continued, and in this species, the ribbing is more closely spaced than on the straight part. The tiny species, S. veterinorus, n. sp., continued the line of small species initiated with S. praecoquus of the basal Carlile. The ventrolateral edges of the aperture are produced outward faintly, foreshadowing the development of lateral lappets by Niobrara time.

Scaphites ferronensis, n. sp., which developed out of S. warreni, continued the trend toward denser ribbing. Many individuals are fairly quadrangular in side view.

Scaphites whitfieldi, n. sp., was derived from $S$. ferronensis by assuming a more quadrangular shape, and the dense ribbing that characterizes the younger part of the older species spread over the entire living chamber and made $S$. whitfieldi the most densely ribbed scaphite of the Carlile. This ribbing is evenly spaced. The suture, which was simplest in S. warreni and $S$. ferronensis, developed slightly longer elements, initiating a trend toward complexity. S. pisinnus, n. sp., which occurs with $S$. whitfieldi, is similar in size to $S$. veterinovus but has a greater lateral extension of the aperture.

Scaphites nigricollensis, n. sp., of the upper part of the Turner sandy member of the Carlile shale descended from $S$. whitfieldi, but is larger and stouter. The density of ventral ribs decreased and the number of primaries increased. The sutures of the two species are similar.

Scaphites corvensis, n. sp., of the Sage Breaks member and uppermost part of the Turner sandy member, continued the trend toward stoutness and increase in size that was initiated with S. nigricollensis. The suture of the younger species has proportionally longer lobes and saddles.

The scaphites of Niobrara age show four lineages. The tiny forms with abnormal apertures produced the new species $S$. auriculatus and $S$. coloradensis. The main line of scaphite development ( $S$. delicatulus through $S$. corvensis) flourished to the end of Niobrara time. A split from this line probably in the uppermost part of the Carlile, gave rise to a very prominent group of scaphites that ranged through the Niobrara and into the basal Montana group (as Desmoscaphites bassleri Reeside). The new species, Scaphites uintensis, S. frontierensis, and $S$. sagensis, of early Niobrara age, represent a fourth line of scaphites, but these species are rare and their relationsip to the other scaphites is unknown.

Scaphites auriculatus is the earliest of the Niobrara species of the praecoquus-veterinovus-pisinnus line of scaphites. The lateral extensions of the aperture developed into prominent lappets directed outward and forward. The suture is simple and shows a bifid first lateral lobe. S. coloradensis of late Niobrara age has a trifid first lateral lobe.

Scaphites corvensis gave rise to $S$. preventricosus, n. sp., by further increase in size, stoutness, and complexity of suture. The living chamber of $S$. preventricosus is less extended from the septate coil. S. ventricosus Meek and Hayden, from the next younger zone, shows till further increase in size, stoutness, and suture complexity. The living chamber is less unrolled and, conversely, more curved. From $S$. ventricosus came $S$. depressus Reeside, which attains a very large size and great stoutness. The living chamber is so little unrolled that only in the more slender forms (S. depressus vars. stantoni Reeside and oregonensis Reeside) is the younger part freed from the septate coil (pl. 15, figs. 1, 7).

Clioscaphites montanensis, n. gen. and sp., descended from $S$. depressus and shows the following trends away from that species: (1) decreased size, (2) less unrolled living chamber, in contact with the septate coil, (3) reduction in size of umbilicus, (4) flattening of flanks, (5) increasing number of ventral ribs on the living chamber in comparison to the primaries, and (6) suture with first lateral lobe transitional from bifid to trifid. The scaphite suture attains its greatest degree of complexity in this species.

Clioscaphites novimexicanus (Reeside) of the youngest Colorado beds was derived from $C$. montanensis by further decrease in size, greater flattening of the flanks, increase in density of ribbing, and perfection of the trifid first lateral lobe. The suture became simplified, thus reversing the trend toward complexity that prevailed since middle Carlile time.

Clioscaphites saxitonianus (McLearn) may represent an evolutionary line that split off from Scaphites ventricosus.

Scaphites mariasensis, n. sp., from beds of early Niobrara age, is the oldest known species of a new line of scaphites that split off from the main line in the late Carlile. The characteristic features of this species are its high, sharp ribs and its fairly simple suture with broad first lateral lobe.

Scaphites impendicostatus, n. sp., descended from $S$. mariasensis by acquiring stronger sculpture with the ribs curving backward in cross section, and on the more stout individuals, becoming flat-crested (pl. 11, figs. $1,2,4,5,7)$. The ventral margin of the aperture is bent outward slightly. A prominent umbilical swelling is present on the large, stout specimens. The suture is as simple as that of its ancestor from the basal Niobrara. The two main branches that make up the first lateral lobe are deeply bifid, making the bifurcating saddles almost as high as the main saddle of the lobe. 
This initiates a new type of suture that persisted through several faunal zones.

Scaphites tetonensis, n. sp., which occurs in the zone next above that of $S$. impendicostatus; shows the following trends away from that species: (1) reduction in stoutness, with loss of the umbilical swelling, (2) reduction in dênsity and strength of ribbing, with the ventral ribs on the older or straight part of the living chamber sparser than on the younger or curved part, and (3) elevation of the ventral extremities of the primary ribs into elongate incipient nodes. Trends initiated with $S$. impendicostatus that persisted through $S$. tetonensis are the outward-bent ventral lip of the aperture and the form of the suture. The suture is a little more incised and continued to have high lateral saddles on the first lateral lobe. These are as high as, or even higher than, the main saddle of the lobe.

Scaphites binneyi Reeside occurs in the zone next above that of $S$. tetonensis. This younger species has ventrolateral tubercles that developed out of the elevated ventral extremities of the primary ribs of S. tetonensis. The suture became slightly more incised and continued to have high lateral saddles on the first lateral lobe. These may be considerable higher than the central saddle of the lobe (pl. 14, fig. 16). S. interjectus Reeside, which occurs with $S$. binneyi, is actually a more advanced form judging by the less unrolled living chamber. The ribbing is denser than on $S$. binneyi but the sutures are similar.

Clioscaphites vermiformis (Meek and Hayden) descended from Scaphites binneyi or S. interjectus. It became so little unrolled that the dorsum of the living chamber was completely in contact with the last septate whorl. The sculpture became considerably stronger, and the ribbing on the internal whorls developed a pronounced forward arching on crossing the venter. The suture is fairly simple and characterized by trifid lobes. The development of trifid lobes out of symmetrically bifid lobes is not clear in this group of scaphites for the change appears to have taken place suddenly. However, if transition faunas are found between the zones of Scaphites binneyi and Clioscaphites vermiformis, the sutures may have asymmetrically bifid first lateral lobes, such as those of Clioscaphites montanensis. An alternate possibility is that the trifid lobe developed directly out of a symmetrically bifid. lobe by the lateral saddles of the lobe having become so high and large as to reduce the inner or apicad prongs of the lobe to a single branch (pl. 14, fig. 16).

An interesting split from $C$. vermiformis is seen in $C$. platygastrus, n. sp., in which the ribs became high and sharp, the primaries lengthened, and the venter flattened. A sharp-ribbed variety of $C$. vermiformis (pl. 18, figs. 20,21 ) is transitional to this species. This new line of scaphites continued into the Telegraph Creek formation of earliest Montana age, where it is represented by an undescribed species with a more compressed form and finer sculpture.

Clioscaphites? choteauensis, n. sp., marks the zone next above that of $C$. vermiformis and shows the following trends away from that species: (1) more rounded venter, (2) denser ribbing, (3) smaller ventrolateral nodes, and (4) more complex suture.

Desmoscaphites erdmanni, n.'sp., occurs at the top of the Colorado shale. It was derived from Clioscaphites? choteauensis by further reduction in the size of the tubercles and by developing nearly uniform spacing of the ventral ribs on the living chamber. The internal whorls have the ribs crossing the venter bent forward as in Clioscaphites vermiformis, but about five constrictions are present on each complete whorl. Clioscaphites? choteauensis, which occurs in the zone between $C$. vermiformis and Desmoscaphites erdmanni, should be transitional between these genera. Unfortunately, the inner whorls have not been seen and the generic assignment of $C$.? choteauensis is only tentative. Desmoscaphites erdmanni gave rise to D. bassleri (Reeside, 1927 c, p. 16 , pl. 21 , figs. $17-21$; pl. 22, figs, 8-12) of the Telegraph Creek formation by further increasing the density of ribbing and developing uniform spacing of the ventral ribs.

Associated with Clioscaphites novimexicanus and Desmoscaphites erdmanni in the latest Colorado beds is a small species, Scaphites leei Reeside, which is completely foreign to the native. Western Interior fauna. This species is characterized by its stout whorls, swollen living chamber, smooth flanks, umbilical and ventrolateral nodes, and simple suture with symmetrically bifid first lateral lobe. Scaphites leei marks the first appearance in the Interior fauna of a prominent group of scaphites (Reeside, 1927c) characterized by $S$. hippocrepis (DeKay) and $S$. aquilaensis Reeside that migrated to America from Europe in Santonian time. These species, rare at first, rapidly dominated the fauna and replaced the Interior scaphites. Only Desmoscaphites bassleri and an undescribed descendent of Clioscaphites platygastrus are known of the native stock in Telegraph Creek time, and by Eagle time, all the scaphites known are of the $S$. hippocrepis-S. aguilaensis group.

The first lateral lobe of the external suture reveals an interesting line of development (fig. 3). In Scaphites warreni and $S$. whitfieldi this lobe is bifid, with each branch consisting of a lower, broadly pointed prong and a lateral bifid prong. This basic pattern is seen in pre-S. warreni species, but in general, it is not as perfectly developed and commonly lacks bilateral symmetry. The next step in the development of this 

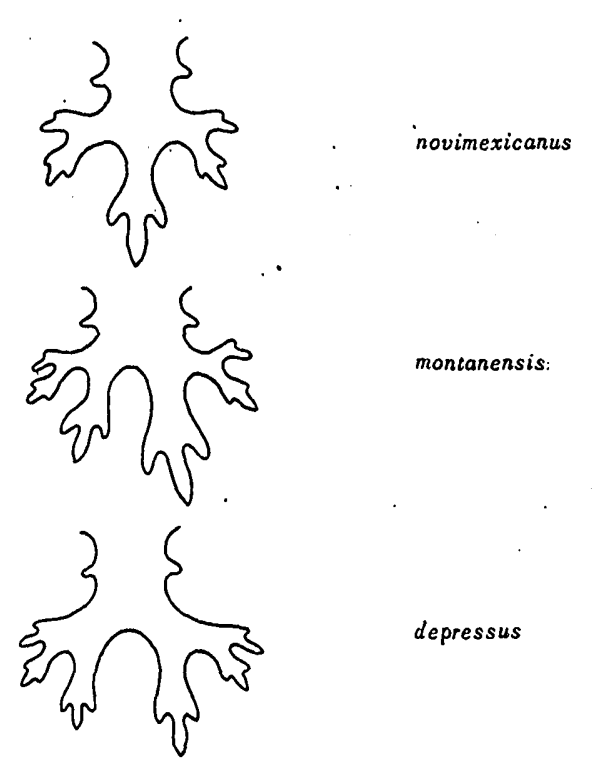

depressus

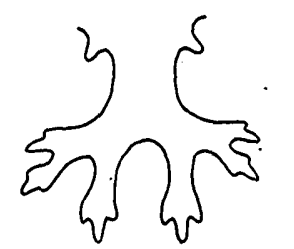

preventricosus

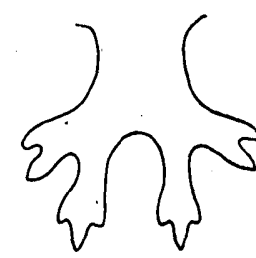

nigricollensis
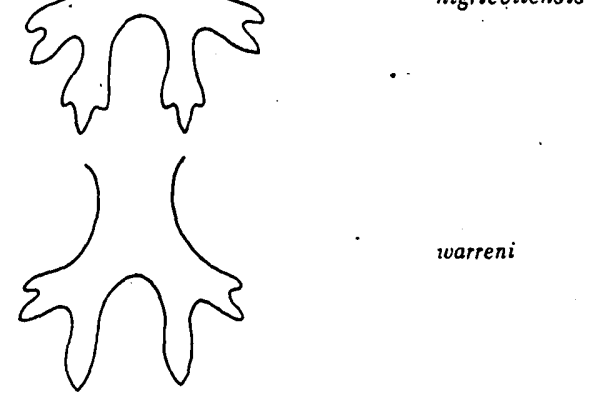

warreni

Finure 3.- Ev volution of the trifl first lateral lobe. The venter would be to the left.

lobe is shown by $S$. nigricollensis in which the apicad prongs became trifid. The next stage was perfected in $S$. preventricosus. The lateral prongs became asymmetrically bifid, with the lower part of each trifid and the upper part smaller and bifid. In general, the lobe was still symmetrical, with a large central saddle and - two smaller equidimensional lateral saddles. In $S$. depressus and varieties the symmetry was largely lost by a slight dwarfing of the ventrad branch of the lobe. This was carried to a great extreme in Clioscaphites montanensis, which had the dorsad branch of the lobe much longer than the ventrad branch. The dorsad saddle became almost as large as the central saddle, and the ventrad saddle was greatly reduced. In the overlying $C$. novimexicanus zone the trifid lobe was perfected by the migration of the trifid prong of the dorsad branch to a central position. The ventrad branch was reduced to the size of the dorsad prong and became symmetrical with it, having its prongs simplified to such an extent that the upper prong was reduced from five points to two.

In summary, the scaphites of the Colorado group reveal several trends in their development that persisted through many faunal zones. The main line of scaphites show, in the Greenhorn and early Carlile, trends toward increase in size, decrease in density of ribbing, and simplification of suture. Scaphites warreni of the basal part of the middle Carlile, marks the maximum simplification of the suture, and initiates a trend toward dense ribbing. This species shows a sudden decrease in size that possibly reflects adverse environmental conditions of that time. After that time, the trend was toward gradual increase in size and in complexity of suture. The maximum size was attained in the zones of Scaphites ventricosus, S. depressus, and Clioscaphites montanensis, of the middle and late Niobrara, and the greatest complexity of suture was reached in Clioscaphites montanensis. After the time of $C$. montanensis, the scaphites decreased in size and the suture became simpler. In the late Carlile the scaphites tended to become less unrolled in the adult stage, and by late Niobrara the whole dorsum of the living chamber was in contact with the outer septate. whorl. This accompanied a reduction in the size of the umbilicus and a tendency for the umbilical wall of the adult living chamber to. be extended over the umbilicus of the septate whorls.

In the late Carlile a split from the main scaphite line gave rise to a group of species at first characterized by peculiar, high, sharp ribs, and later by the development of a row of ventrolateral tubercles. These species paralleled the main line of scaphites in becoming larger, less unrolled, and in developing a suture with trifid lobes. However, the suture never reached the degree of complexity seen in Clioscaphites montanensis.

A group of tiny species represents another line of development beginning with Scaphites praecoquus of the basal Carlile. This species, which is very closely related to $S$. larvaeformis; has a nearly normal aperture, but the later species showed progressively greater forward extension of the lateral margins of the aperture. By early Niobrara . time, conspicuous pointed lateral lappets were developed and, by the late Niobrara, the suture developed trifid lobes.

\section{GEOGRAPHIC DISTRIBUTION}

The occurrence of the scaphites of the Colorado group by state and locality is indicated in the insert (facing p. 12), and the localities are indicated on figure 4. 


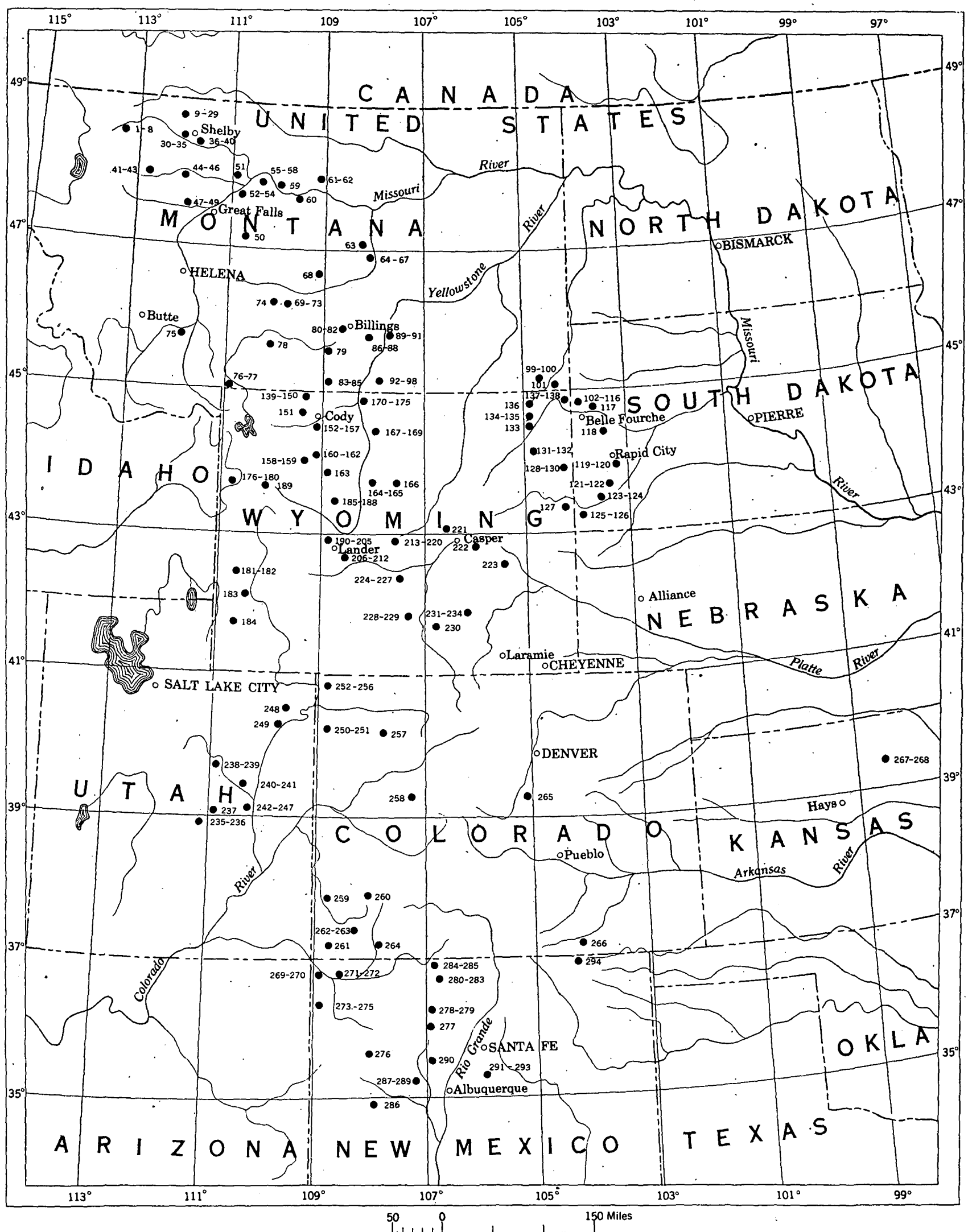

Figure 4.-Index map showing localities of collections from rocks of Colorado age. Numbers refer to the table of distribution and to the detailed description of localities on 

The individual localities are described in the following list.

Localities at which scaphite cephalopods were collected from rocks of the Colorado group

\begin{tabular}{|c|c|c|}
\hline $\begin{array}{c}\text { No. } \\
\text { on } \\
\text { fig. } \\
4\end{array}$ & $\begin{array}{l}\text { U.S.Q.S. } \\
\text { Miesozoic } \\
\text { locality } \\
\text { number }\end{array}$ & $\begin{array}{l}\text { Collector, year of collection, description of } \\
\text { locality, and stratigraphic assignment }\end{array}$ \\
\hline 1 & 7127 & $\begin{array}{l}\text { M. R. Campbell and 'J. W. Stanton, 1911. About } 2.5 \text { miles } \\
\text { west-northwost of Summit, Glacier National Park, Mont. } \\
\text { Colorado shale. }\end{array}$ \\
\hline 2 & 7128 & $\begin{array}{l}\text { M. R. Campbell, J. Hoats, and T. W. Stanton, 1911. On south- } \\
\text { wost slopo of Summit Mountain, Glacier National Park, Mont. } \\
\text { Colorado shalo. }\end{array}$ \\
\hline 3 & 7129 & $\begin{array}{l}\text { T. W. Stanton, 1911. Great Northern Railroad cut three-fourths } \\
\text { mile northeast of Summit, Glacier National Park, Mont. } \\
\text { Colorado shale. }\end{array}$ \\
\hline 4 & 6551 & $\begin{array}{l}\text { M. R. Campbell, 1910. Railroad cut } 2.5 \text { miles west of Lubec, } \\
\text { Glacier National Park, Mont. Colorado shale. }\end{array}$ \\
\hline 5 & 7131 & $\begin{array}{l}\text { T. W. Stanton, 1911. East slope of Squaw Mountain north of } \\
\text { Jubec, Clacier National Park, Mont. Colorado shale. }\end{array}$ \\
\hline 6 & 7162 & $\begin{array}{l}\text { M. R. Campbell and J. Hoats, 1911. Ridge south of North Fork } \\
\text { of Cut Bank Creek, Glacier National Park, Mont. Colorado } \\
\text { shale. }\end{array}$ \\
\hline 7 & 7137 & $\begin{array}{l}\text { M. R. Cumpbell, J. Hoats, and T. W. Stanton, } 1911 \text {. North side } \\
\text { of Two Medicine Rivor, } 9 \text { miles south of Browning, in the NW14 } \\
\text { sec. } 27, T .31 \text { N., R. } 11 \text { W., Glacier County, Mont. Colorado } \\
\text { shule. }\end{array}$ \\
\hline 8 & 7142 & $\begin{array}{l}\text { M. R. Campbell and T. W. Stanton, 1911. South side of Two } \\
\text { Medicine River, in the NW1/4 sec. } 26 . \text { T. } 31 \text { N., R. } 11 \text { W., } \\
\text { Glacier County, Mont. Colorado shale. }\end{array}$ \\
\hline 9 & 9303 & $\begin{array}{l}\text { L. S. Kompher, 1915. Four miles west of Sunburst, in sec. } 9 \text {, } \\
\text { T. } 36 \text { N., R. } 3 \text { W., Toole County, Mont. Colorado shale, } \\
\text { upper part. }\end{array}$ \\
\hline 10 & 9301 & $\begin{array}{l}\text { T. S. Kempher, } 1915 \text {.. Four miles west of Sunburst, in sec. } 16 \text {, } \\
\text { 'T. } 36 \text { N., R. } 3 \text { W., Toole County, Mont.' Colorado shale, } \\
\text { near top. }\end{array}$ \\
\hline 11 & 8048 & $\begin{array}{l}\text { T. W. Stanton, } 1912 \text {. Nine miles west of Kevin, in sec. } 29, \text { T. } 35 \\
\text { N., R. } 4 \text { W., Toole County, Mont. Colorado shale, near top. }\end{array}$ \\
\hline 12 & 20299 & $\begin{array}{l}\text { C. E. Erdmann and J. T. Gist, 1944. Five miles northwest of } \\
\text { Kevin, in the NE } 14 \text { NW } 34 \text { sec. } 24, T \text {. } 35 \text { N., R. } 4 \text { W., Toole } \\
\text { County, Mont. Colorado shale, } 101 \text { feet below top. }\end{array}$ \\
\hline 13 & 21.667 & $\begin{array}{l}\text { C. E. Erdmann, R. W. Imlay, and J. B. Reeside, Jr., 1944. Five } \\
\text { miles northwest of Kevin, in the NE. corner NW34 sec. } 24 \text {, } \\
\text { I. } 35 \text { N., R. } 4 \text { W., Toole County, Mont. Colorado shale, } 23 \\
\text { feet below top. }\end{array}$ \\
\hline 14 & 8605 & $\begin{array}{l}\text { Eugeno Stebinger, } 1913 \text {. Five miles northwest of Kevin, in the } \\
\text { NWW sec. } 24, \text { T. } 35 \text { N., R. } 4 \text { W., Toole County, Mont. Colorado } \\
\text { shale, upper part. }\end{array}$ \\
\hline 15 & 20291 & $\begin{array}{l}\text { C. W. Erdmant and J. T. Gist, } 1944 \text {. Four miles north of Kevin, } \\
\text { in the SW34 sec. } 3 \text {, T. } 35 \text { N., R. } 3 \text { W., Toole County, Mont. } \\
\text { Colorado shalo, } 379 \text { to } 381 \text { feet below top. }\end{array}$ \\
\hline 10 & 20282 & $\begin{array}{l}\text { O. E. Erdmann and J. T. Gist, 1944. Same locality as } 20291 \text {. } \\
\text { Colorado shale, } 381 \text { to } 388 \text { feet below top. }\end{array}$ \\
\hline 17 & 20295 & $\begin{array}{l}\text { C. W. Erdmann and J. T. Gist, 1944. Same locality as } 20291 \text {. } \\
\text { Colorado shalo, } 364 \text { fect below top. }\end{array}$ \\
\hline 18 & 21483 & $\begin{array}{l}\text { B. R. Alto, W. A. Cobban, and C. T. Moore, } 1949 \text {. Five miles } \\
\text { northwest of Kevin, in the SE1/4NE1/4 sec. } 4, T .35 \text { N., R. } 3 \text { W., } \\
\text { Toole County, Mont. Colorado sliale, in ferruginous concre- } \\
\text { tions } 306 \text { to } 392 \text { feet below top. }\end{array}$ \\
\hline 10 & 11995 & $\begin{array}{l}\text { A. J. Collier, } 1923 \text {. Three miles northwest of Kovin, in sec. } 17 \text {, } \\
\text { T. } 35 \text { N., R. ' } 3 \text { W., Toole County, Mont. Colorado shale, } 20 \\
\text { feet below top. }\end{array}$ \\
\hline 20 & 11974 & $\begin{array}{l}\text { A. J. Collier, 1923. Three miles north of Kevin, in the NE1/4 sec. } \\
15 \text {, 'T. } 35 \text { N., R. } 3 \text { W., Toole County, Mont. Colorado shale, } \\
500 \text { to } 580 \text { foet below top. }\end{array}$ \\
\hline 21 & 20290 & $\begin{array}{l}\text { C. E. Erdmann and J. T. Gist, } 1944 \text {. Three miles north of Kovin, } \\
\text { in the SW } 1 / 4 \text { NW } 1 / 4 \text { NE } 1 / 4 \text { sec. } 15, T .35 N \text {, , R. } 3 \text { W., Toole County, } \\
\text { Mont. Colorado shale, } 513 \text { to } 517 \text { feet below top. }\end{array}$ \\
\hline 22 & 20298 & $\begin{array}{l}\text { C. I. Erdmann and J. T. Gist, 1944. Same locality as } 20296 \text {. } \\
\text { Colorado shale, } 527 \text { to } 531 \text { feet below top. }\end{array}$ \\
\hline 23 & 20297 & $\begin{array}{l}\text { C. E. Erdmann and J. T. G ist, } 1944 \text {. Three miles north of Kevin, } \\
\text { in the NW1/4 sec. } 15, \text { T. } 35 \mathrm{~N} \text {., R. } 3 \text { W., Toole County, Mont. } \\
\text { Colorado shale, } 528 \text { to } 532 \text { feet below top. }\end{array}$ \\
\hline 24 & 20280 & $\begin{array}{l}\text { C. E. Erdmann and J. T. Gist, 1944. Three miles north of Kevin, } \\
\text { in the SW1/4NE1/4NW1/4 sec. } 15, \text { T. } 35 \text { N., R. } 3 \text { W., Toole County, } \\
\text { Mont. Colorado shale, about } 600 \text { feet below top. }\end{array}$ \\
\hline
\end{tabular}

\begin{tabular}{|c|c|c|}
\hline $\begin{array}{l}\text { No. } \\
\text { on } \\
\text { fig. } \\
4\end{array}$ & $\begin{array}{l}\text { U.S.G.S. } \\
\text { Mesozoic } \\
\text { locality } \\
\text { number }\end{array}$ & $\begin{array}{l}\text { Collector, year of collection, description of } \\
\text { locality, and stratigraphic assignment }\end{array}$ \\
\hline 25 & 20911 & $\begin{array}{l}\text { C. E. Erdinann, 1947. Five miles north-northwest of Oilmont, } \\
\text { in the NW1/4 NW1/4NW1/4 sec. } 12, \mathrm{~T} .35 \mathrm{~N} ., \mathrm{R} .2 \mathrm{~W} \text {., Toole } \\
\text { County, Mont. Colorado shale, pebbly sandstone } 651 \text { feet } \\
\text { below top. }\end{array}$ \\
\hline 26 & 21429 & $\begin{array}{l}\text { W. A. Cobban, } 1943 \text {. One mile west of Oilmo nt, In the NW1/4 } \\
\text { NW1/4 sec. } 3 \text {, T. } 34 \text { N., R. } 2 \text { W., Toole County, Mont. Colorado } \\
\text { shale, in ferruginous concretions about } 800 \text { feet below top of } \\
\text { Colorado. }\end{array}$ \\
\hline 27 & 21807 & $\begin{array}{l}\text { W. A. Cobban, } 1949 \text {. Ten miles east of Oilmont in the SE } 1 / 4 \text { SE } 1 / 4 \\
\text { SE } 1 / 4 \text { sec. } 32 \text {, T. } 35 \text { N., R. } 1 \text { E., Toole County, Mont. Colorado } \\
\text { shale, about } 675 \text { feet below top. }\end{array}$ \\
\hline 28 & 22130 & $\begin{array}{l}\text { M. E. Porter, 1948. A bout } 2 \text { miles north of Ethridge, Toole Coun- } \\
\text { ty, Mont. Colorado shale near top. }\end{array}$ \\
\hline 29 & 11987 & $\begin{array}{l}\text { A. J. Collier, 1923. Three miles northwest of Shelby, in sec. } 8 \text {, } \\
\text { T. } 32 \text { N., R. } 2 \text { W., Toole County, Mont. }\end{array}$ \\
\hline 30 & 21419 & $\begin{array}{l}\text { W. A. Cobban, 1948. Eight miles wes } \mathrm{t} \text { of Shelby, at head of } \\
\text { ravine } 3 \text { miles north of Marias River, } i_{\text {n the NE1/4 sec. } 31, T .32} \\
\text { N., R. } 3 \text { W., Toole County, Mont. Colorado shale, } 10 \text { feet } \\
\text { below top. }\end{array}$ \\
\hline 31 & 11989 & $\begin{array}{l}\text { A. J. Collier, 1923. North side o }- \text { Marias River, sec. 3, T. 31. N., } \\
\text { R. } 4 \text { W., Toole County, Mont. Colorado shale. }\end{array}$ \\
\hline 32 & 21412 & $\begin{array}{l}\text { B. R. Alto and K. Holmes, } 1948 \text {. A bout } 11.5 \text { miles southwest of } \\
\text { Shelby, in the SW1/4SW1/4 sec. } 14, T \text {. } 31 \text { N., R. } 4 \text { W., Pondera } \\
\text { County, Mont. Colorado shale, about } 250 \text { feet below top. }\end{array}$ \\
\hline 33 & 21425 & $\begin{array}{l}\text { W. A. Cobban, } 1940 \text {. East bank of Marias River, } 11 \text { miles south- } \\
\text { west of Shelby, in the W1/2NE } 1 / 4 \text { SE } 1 / 4 \text { sec. } 14, T .31 \text { N., R. } 4 \text { W., } \\
\text { Toole County, Mont. Colorado shale, } 234 \text { to } 252 \text { feet below top. }\end{array}$ \\
\hline 34 & 21421 & $\begin{array}{l}\text { W. A. Cobban, } 1935 \text {. North bank of Marias River, } 5.5 \text { miles south } \\
\text { of Shelby, in the NE1/4 sec. } 20, T .31 \text { N., R. } 2 \text { W., Toole County, } \\
\text { Mont. Colorado shale, } 617 \text { to } 634 \text { feet below top. }\end{array}$ \\
\hline 35 & 21422 & $\begin{array}{l}\text { W. A. Cobban, } 1935 \text {. Same locality as } 21421 \text {. Colorado shale, } \\
514 \text { to } 525 \text { feet below top. }\end{array}$ \\
\hline 36 & 11979 & $\begin{array}{l}\text { A. C. Collier, 1923. One mile below railroad bridge across Marias } \\
\text { River, in sec. 4, T. } 30 \text { N., R. } 1 \text { W., Toole County, Mont. Colo- } \\
\text { rado shale. }\end{array}$ \\
\hline 37 & 22166 & $\begin{array}{l}\text { K. H. Holmes, 1949. Nine miles southiest of Devon, in the } \\
\text { SE1/4SW1/4 sec. } 16 \text {, T. } 30 \text { N., R. I E., Toole County, Mont. } \\
\text { Colorado shale, } 468 \text { feet below top. }\end{array}$ \\
\hline 38 & 22149 & $\begin{array}{l}\text { K. H. Holmes, } 1949 \text {. Eleven miles south of Devon, in the SE1/4 } \\
\text { SW1/4 sec. } 34, T .30 \text { N., R. } 2 \text { E., Toole County, Mont. Colorado } \\
\text { shale, } 165 \text { feet below top. }\end{array}$ \\
\hline 39 & 22161 & $\begin{array}{l}\text { K. H. Holmes, } 1949 \text {. Marias River, } 9 \text { miles south of Devon, in } \\
\text { the NE1/4SW1/4 sec. } 22 \text {, T. } 30 \text { N., R. } 2 \text { E., Toole County, Mont. } \\
\text { Colorado shale, } 330 \text { feet below top. }\end{array}$ \\
\hline 40 & 22164 & $\begin{array}{l}\text { K. H. Holmes, } 1949 \text {. Eight miles south-southwest of Devon, in } \\
\text { the NW1/4SW1/4 sec. } 18 \text {, T. } 30 \text { N., R. } 2 \text { E., Toole County, Mont. } \\
\text { Colorado shale, } 484 \text { feet below top. }\end{array}$ \\
\hline 41 & 9853 & $\begin{array}{l}\text { M. I. Goldman, } 1916 \text {. Sheep Creek, in sec. } 34, \text { T. } 28 \text { N., R. } 8 \\
\text { W., Pondera County, Mont. Colorado shale. }\end{array}$ \\
\hline 42 & 9856 & $\begin{array}{l}\text { M. I. Goldman and E. Stebinger, } 1916 \text {. Southeast slope of Ante- } \\
\text { lope Butte, in sec. } 28 \text {, T. } 26 \text { N., R. } 8 \text { W., Teton County, Mont. } \\
\text { Colorado Shale. }\end{array}$ \\
\hline 43 & 9890 & $\begin{array}{l}\text { Eugene Stebinger, } 1916 . \text { On Willow Creek, in the NW1/4 sec. } 19 \text {, } \\
\text { 'T. } 24 \text { N., R. } 7 \text { W., Teton County, Mont. Cclorado shale, near } \\
\text { top. }\end{array}$ \\
\hline 44 & 4023 & $\begin{array}{l}\text { W. R. Calvert, } 1906 \text {. About half a mile northeast of Choteau, in } \\
\text { sec. } 19, \text { T. } 24 \text { N., R. } 4 \text { W., Teton County, Mont. Colorado } \\
\text { shale, upper part. }\end{array}$ \\
\hline 45 & 11971 & $\begin{array}{l}\text { A. J. Collier, } 1923 \text {. North side of Teton River above Dent bridge, } \\
\text { in sec. } 34, \text { T. } 25 \text { N., R. } 3 \text { W., Teton County, Mont. Colorado } \\
\text { shale, about } 500 \text { feet below top. }\end{array}$ \\
\hline 46 & 21806 & $\begin{array}{l}\text { W. A. Cobban and C. T. Moore, } 1949 \text {. Two miles southeast of } \\
\text { Power, in the W1 } 1 / 2 \text { sec. } 34, \text { T. } 23 \text { N., R. } 1 \text { W., Teton County, } \\
\text { Mont. Colorado shale, about } 900 \text { feet above base. }\end{array}$ \\
\hline 47 & · 21372 & $\begin{array}{l}\text { W. A. Cobban and R. W. Imlay, 1946. Two miles north of Fort } \\
\text { Shaw, in the S1/3 secs. } 35 \text { and } 36, T .21 \mathrm{~N} \text {., R. } 2 \text { W., Cascade } \\
\text { County, Mont. Colorado shale, } 184 \text { to } 208 \text { feet above top of } \\
\text { calcareous shale of Greenhorn age. }\end{array}$ \\
\hline
\end{tabular}

$939551-51-2$ 
Localities at which scaphite cephalopods were collected from rocks of the Colorado group-Continued

\begin{tabular}{|c|c|c|}
\hline $\begin{array}{l}\text { No. } \\
\text { on } \\
\text { fig. } \\
4\end{array}$ & $\begin{array}{l}\text { U.S.G.S. } \\
\text { Mesozoic } \\
\text { locality } \\
\text { number }\end{array}$ & $\begin{array}{l}\text { Collector, year of collection, description of } \\
\text { locality, and stratigraphic assignment }\end{array}$ \\
\hline 48 & 21373 & $\begin{array}{l}\text { W. A. Cobban, 1942. Same locality as } 21372 \text {. Colorado shale, } \\
202 \text { to } 207 \text { feet above calcareous shale of Greenhorn age. }\end{array}$ \\
\hline 49 & 21375 & $\begin{array}{l}\text { W. A. Cobban, } 1942 \text {. Same locality as } 21372 \text {. Colorado shale, } \\
238 \text { to } 255 \text { feet above calcareous shale of Greenhorn age. }\end{array}$ \\
\hline 50 & 3956 & $\begin{array}{l}\text { C. A. Fisher, 1906. Six miles northwest of Geyser, Judith Basin } \\
\text { County, Mont. Colorado shale. }\end{array}$ \\
\hline 51 & 16201 & $\begin{array}{l}\text { C. B. Hunt, 1932. T. } 26 \text { N., R. } 7 \text { E., Chouteau County, Mont. } \\
\text { Colorado shale, } 270 \text { feet below base of Virgelle sandstone. }\end{array}$ \\
\hline 52 & 1400 & T. W. Stanton, 1894. Fort. Benton, Mont. Colorado shale. \\
\hline 53 & 152 & J. B. Marcou, 1883. Fort Benton, Mont. Colorado shale. \\
\hline 54 & 1403 & $\begin{array}{l}\text { T. W. Stanton and W. H. Weed, } 1894 \text {. Missouri River, about } 10 \\
\text { miles below Fort Benton. Colorado shale. }\end{array}$ \\
\hline 55 & $\ldots$. & $\begin{array}{l}\text { John Pearsall, 1862. Chippewa Point, on the Missouri River } 20 \\
\text { miles [presumably river miles] below Fort Benton, Mont. } \\
\text { Colorado shale, upper part. }\end{array}$ \\
\hline 56 & 154 & $\begin{array}{l}\text { J. B. Marcou, 1883. Missouri River } 3 \text { miles below mouth of } \\
\text { Marias River. Colorado shale. }\end{array}$ \\
\hline 57 & 1404 & $\begin{array}{l}\text { T. W. Stanton and W. H. Weed, 1894. Missouri River } 4 \text { or } 5 \\
\text { miles below mouth of Marias River, Mont. Colorado shale, } \\
\text { upper part. }\end{array}$ \\
\hline 58 & 8968 & $\begin{array}{l}\text { E. R. Lloyd, W. T. Thom, Jr., and W. B. Wilson, } 1914 \text {. One } \\
\text { mile south of Virgelle, in the NE1/4 sec. } 24, \text { T. } 26 \text { N., R. } 11 \text { E. } \\
\text { Chouteau County, Mont. Colorado shale. }\end{array}$ \\
\hline 59 & 2852 & $\begin{array}{l}\text { J. B. Hatcher, and T. W. Stanton, } 1903 . \text { Missouri River near } \\
\text { Eagle Butte, Chouteau County, Mont. Colorado shale. }\end{array}$ \\
\hline 60 & 1407 & $\begin{array}{l}\text { T. W. Stanton and W. H. Weed, 1894. On Missouri River } 5 \text { or } 6 \\
\text { miles below mouth of Arrow Creek, Fergus County, Mont. } \\
\text { Colorado shale, upper part. }\end{array}$ \\
\hline 61 & 2839 & $\begin{array}{l}\text { J. B. Hatcher and T. W. Stanton, } 1903 \text {. Thirteen miles above } \\
\text { mouth of Cow Creek, Blaine County, Mont. Colorado shale, } \\
\text { upper part. }\end{array}$ \\
\hline 62 & $2847 \mathrm{a}$ & $\begin{array}{l}\text { J. B. Hatcher, and T. W. Stanton, 1903. Thirteen miles above } \\
\text { mouth of Cow Creek [probably the W1/2 sec. 4, T. } 25 \text { N., R. } 21 \text { E., } \\
\text { Blaine County], Mont. Colorado shale. }\end{array}$ \\
\hline 63 & 7904 & $\begin{array}{l}\text { C. F. Bowen, } 1912 \text {. Southwest flank of West dome of Cat Creek } \\
\text { anticline, in see. 17, T. } 15 \text { N., R. } 29 \text { E., Petroleum County, } \\
\text { Mont. Colorado shale, near top. }\end{array}$ \\
\hline 64 & 21399 & $\begin{array}{l}\text { W. A. Cobban, } 1948 \text {. Half a mile west of bridge over Musselshell } \\
\text { River near Mosby, in the SE1/4 sec. } 10 \text {, T. } 14 \text { N., R. } 30 \text { E., } \\
\text { Petroleum County, Mont. Colorado shale, in ferruginous } \\
\text { concretions, } 99 \text { to } 115 \text { feet above top of Mosby sandstone } \\
\text { member. }\end{array}$ \\
\hline 65 & 21401 & $\begin{array}{l}\text { W. A. Cobban, 1948. Three miles east of Mosby, in the SW1/4 } \\
\text { sec. } 8, \text { T. } 14 \text { N., R. } 31 \text { E., Garfleld County, Mont. Colorado } \\
\text { shale, } 5 \text { feet above so-called Sage Hen limestone member. }\end{array}$ \\
\hline 66 & 21402 & $\begin{array}{l}\text { W. A. Cobban, } 1948 \text {. Three miles east of Mosby, in the N1/2 } \\
\text { sec. } 8, \text { T. } 14 \text { N., R. } 31 \mathrm{E} \text {., Garfield County, Mont. Colorado } \\
\text { shale, } 300 \text { feet below top of calcareous shale of Niobrara age. }\end{array}$ \\
\hline 67 & 21403 & $\begin{array}{l}\text { W. A. Cobban, } 1948 \text {. Same locality as } 21402 \text {. Colorado shale, } \\
251 \text { to } 280 \text { feet below top of calcareous shale of Niobrara age. }\end{array}$ \\
\hline 68 & 8828 & $\begin{array}{l}\text { C. E. Lesher, } 1914 \text {. Sec. } 19 \text {, T. } 9 \text { N., R. } 22 \text { E., Golden Valley } \\
\text { County, Mont. Colorado shale. }\end{array}$ \\
\hline 69 & 2894 & $\begin{array}{l}\text { M. S. Farr, J. B. Hatcher, A. C. Silberling, and T. W. Stanton, } \\
\text { 1903. Near Crawford's Ranch, in the NW1/4 T. } 6 \text { N., R. } 16 \text { E., } \\
\text { Wheatland County, Mont. Colorado shale, upper part. }\end{array}$ \\
\hline 70 & 2895 & $\begin{array}{l}\text { M. S. Farr, J. B. Hatcher, A. C. Silberling, and T. W. Stanton, } \\
\text { 1903. Near Crawford's Rranch on Mud Creek, Wheatland } \\
\text { County, Mont. Colorado shale, near top. }\end{array}$ \\
\hline 71 & 2896 & $\begin{array}{l}\text { M. S. Farr, J. B. Hatcher, A. C. Silberling, and T. W. Stanton, } \\
\text { 1903. Mud Creek about one mile northeast of Crawford's } \\
\text { Ranch, Wheatland County, Mont. Colorado shale. }\end{array}$ \\
\hline 72 & 4655 & $\begin{array}{l}\text { R. W. Stone, 1907. East bank of American Fork, in sec. } 23, \text { T. } 7 \\
\text { N., 15 E., Wheatland County, Mont. Colorado shale. }\end{array}$ \\
\hline 73 & 4656 & $\begin{array}{l}\text { R. W. Stone, } 1907 \text {. Ten miles south of Harlowton, in sec. 12, } \\
\text { T. } 6 \text { N., R. } 15 \text { E., Wheatland County, Mont. Colorado shale. }\end{array}$ \\
\hline 74 & 4588 & $\begin{array}{l}\text { R. W. Stone, } 1907 . \text { Big Elk Creek near bridge, in the NE1/4 } \\
\text { sec. } 36, T \text {, } 7 \text { N., R. } 13 \text { E., Wheatland County, Mont. Colo- } \\
\text { rado shale. }\end{array}$ \\
\hline 75 & 1663 & $\begin{array}{l}\text { A. C. Peale, 1891. Opposite Jefferson Canyon. [Probably about } \\
5 \text { miles west of Sappington, Madison County, Mont.] Colorado } \\
\text { shale. }\end{array}$ \\
\hline 76 & - & $\begin{array}{l}\text { F. V. Hayden, i871. Cinnabar Mountain, near Gardiner, Park } \\
\text { County, Mont. Colorado shale, upper part. }\end{array}$ \\
\hline
\end{tabular}

\begin{tabular}{|c|c|c|}
\hline $\begin{array}{l}\text { No. } \\
\text { on } \\
\text { fig. } \\
4\end{array}$ & $\begin{array}{l}\text { U.S.G.S. } \\
\text { Mesozoic } \\
\text { locality } \\
\text { number }\end{array}$ & $\begin{array}{l}\text { Collector, year of collection, description of } \\
\text { locality, and stratigraphic assignment }\end{array}$ \\
\hline 77 & 22131 & $\begin{array}{l}\text { W. A. Cobban, 1937. Cinnabar Mountain, } 4.5 \text { miles west- } \\
\text { northwest of Gardiner, Park County, Mont. Colorado shale, } \\
\text { near top. }\end{array}$ \\
\hline 78 & 5608 & $\begin{array}{l}\text { W. R. Calvert and T. W. Stanton, 1908. North side of Boulder } \\
\text { Creek near old McLeod P. O., Sweet Grass County, Mont. } \\
\text { Colorado shale, near top. }\end{array}$ \\
\hline
\end{tabular}

$$
80
$$$$
8
$$

$$
\begin{array}{r|r}
85 & 5051 \\
86 & 10897 \\
\hline
\end{array}
$$$$
87
$$

\author{
90
}

92 
Localities at which scaphite cephalopods were collected from rocks of the Colorado group-Continued

\begin{tabular}{|c|c|c|}
\hline $\begin{array}{l}\text { No. } \\
\text { on } \\
\text { fig. } \\
4\end{array}$ & $\begin{array}{l}\text { U.S.G.S. } \\
\text { Miesozoic } \\
\text { locrlity } \\
\text { number }\end{array}$ & $\begin{array}{l}\text { Collector, year of collection, description of } \\
\text { locality, and stratigraphic assignment }\end{array}$ \\
\hline 103 & 21182 & $\begin{array}{l}\text { W. A. Cobban. 1947. Fivo miles north of Belle Fourche. in the } \\
\text { SW1/4SE1/4SW1/4 sec. } 11, T \text { T. } 9 \text { N., R. } 2 \text { E., Butte County, S. } \\
\text { Dak. Carlile shale, in ferruginous concretions } 57 \text { to } 81 \text { feet above } \\
\text { base. }\end{array}$ \\
\hline 104 & 21.183 & $\begin{array}{l}\text { W. A. Cobban, 1947. Same locality as } 21182 \text {. Carlile shale, } 58 \\
\text { to } 05 \text { fect above base. }\end{array}$ \\
\hline 105 & 21184 & $\begin{array}{l}\text { W. A. Cobban, 1947. Samo locality as } 21182 \text {. Carlile shale, } 65 \\
\text { fcot abovo base. }\end{array}$ \\
\hline$\cdot 106$ & 21185 & $\begin{array}{l}\text { W. A. Cobban, 1947. Same locality as } 21182 \text {. Carlile shale, } 66 \\
\text { to } 78 \text { fect abovo base. }\end{array}$ \\
\hline 107 & 21186 & $\begin{array}{l}\text { W. A. Cobban, } 1947 \text {. Same locality as } 21182 \text {. Carlile shale, } 81 \\
\text { feet above base. }\end{array}$ \\
\hline 108 & 21187 & $\begin{array}{l}\text { W. A. Cobban, } 1947 \text {. Fivo miles north of Belle Fourche, in the } \\
\text { SW1/4SE1/4SW1/4 sec. } 11 \text {, T. } 9 \text { N., R. } 2 \text { E., Butte County, S. } \\
\text { Duk. Carlile shale, from large calcareous concretions } 14.5 \text { feet } \\
\text { above base of Turner sandy member. }\end{array}$ \\
\hline 100 & 21188 & $\begin{array}{l}\text { W. A. Cobban, 1947. Samo locality as } 21187 \text {. Carlile shale, from } \\
\text { calcareous concrotions } 36 \text { feet above base of Turner sandy } \\
\text { nember. }\end{array}$ \\
\hline 110 & 21189 & $\begin{array}{l}\text { W. A. Cobban, 1947. Six miles north of Belle Fourche, in the } \\
\text { N1/2 sec. } 10 \text {, '1. } 9 \text { N., R. } 2 \text { E., Butte County, S. Dak. Carlile } \\
\text { shalc, } 164 \text { fect above base. }\end{array}$ \\
\hline 111 & 21190 & $\begin{array}{l}\text { W. A. Cobban, 1947. Same locality as } 21189 \text {. Carlile shale, } 194 \\
\text { to } 202 \text { feet abovo base. }\end{array}$ \\
\hline 112 & 21194 & $\begin{array}{l}\text { W. A. Cobban, } 1947 \text {. Same locality as } 21189 \text {. Carlile shale, } 251 \\
\text { to } 264 \text { feet above base. }\end{array}$ \\
\hline 113 & 21195 & $\begin{array}{l}\text { W. A. Cobban, 1947. Six miles north of Belle Fourche, in the } \\
\text { NE } 1 / 4 \text { NIE1 } 1 / 4 \text { NW1/4 sec. } 10, T .9 \text { N., R. } 2 \text { E., Butte County, S. } \\
\text { Dak. Carlile shale, } 272 \text { to } 274 \text { feet above base. }\end{array}$ \\
\hline 114 & 21108 & $\begin{array}{l}\text { W. A. Cobban, } 1947 \text {. Same locality as } 21195 \text {. Carlile shale, } 293 \\
\text { feet above base. }\end{array}$ \\
\hline 115 & 21100 & $\begin{array}{l}\text { W. A. Cobban, } 1947 \text {. Same locality as } 21195 \text {. Carlile shale, } 333 \\
\text { to } 336 \text { feet above base. }\end{array}$ \\
\hline 110 & 12734 & $\begin{array}{l}\text { W. W. Rubey, 1924. Five miles north of Belle Fourche, S. Dak. } \\
\text { Carlilo shale, lower part. }\end{array}$ \\
\hline 117 & 21428 & $\begin{array}{l}\text { W. A. Cobban, 1946. Three miles northeast of Fruitdale, in } \\
\text { center of sec. } 33, \text { T. } 9 \text { N., R. } 4 \text { E., Butte County, S. Dak. Carlile } \\
\text { shale, about } 60-70 \text { feet above base. }\end{array}$ \\
\hline 118 & 12176 & $\begin{array}{l}\text { Mrs. C. J. Haas, 1023. Near Whitewood, Lawrence County, } \\
\text { S. Dak. Carlile shale. }\end{array}$ \\
\hline 110 & 21765 & $\begin{array}{l}\text { W. A. Cobban, 1948. Nine miles south-southeast of Rapid City, } \\
\text { in the NE1/4 sec. } 22 \text {, T. } 1 \text { S., R. } 8 \text { W., Pennington County, S. } \\
\text { Dak. Sage Breaks member of Carlile shale, } 42 \text { to } 46 \text { feet above } \\
\text { bnse. }\end{array}$ \\
\hline 120 & 21053 & $\begin{array}{l}\text { W. A. Cobban, 1946. Nine miles south-southeast of Rapid City, } \\
\text { in the NW1/4 sec. } 21 \text {, T. } 1 \text { S., R. } 8 \text { E., Pennington County, S. } \\
\text { Dak. Carlilo shale, near base. }\end{array}$ \\
\hline 121 & 18872 & $\begin{array}{l}\text { N. II. Darton, 1888. Two miles southeast of Fairburn, Custer } \\
\text { County,'s. Dak. Carlile shale, lower part. }\end{array}$ \\
\hline 122 & 12042 & $\begin{array}{l}\text { J. B. Recside, Jr. and W. W. Rubey, } 1924 \text {. Two miles south of } \\
\text { Fairburn, in sce. } 31 \text {, T. } 4 \text { S., R. } 8 \text { E., Custer County, S. Dak. } \\
\text { Carlile shale, calcareous concretions } 90 \text { feet below top of lower } \\
\text { member. }\end{array}$ \\
\hline 123 & 10383 & $\begin{array}{l}\text { T. W. Stanton, 1920. Halt a mile southwest of Buffalo Gap, } \\
\text { Custer County, S. Dak. Carlile shale, Turner sandy member. }\end{array}$ \\
\hline 124 & 10382 & $\begin{array}{l}\text { T. W. Stanton, } 1920 \text {. About } 5 \text { miles southeast of Hot Springs } \\
\text { Fall Rlver County, S. Dak. Carlile shale, near base. }\end{array}$ \\
\hline 125 & $\ldots$ & $\begin{array}{l}\text { F. V. Hayden, 1857. Southern base of Black Hills, S. Dak. } \\
\text { Carlilo shale. }\end{array}$ \\
\hline 120 & .13610 & $\begin{array}{l}\text { W. W. Rubey, 1926. Five miles south of Edgemont, in the NW1/4 } \\
\text { sec. } 30 \text {, T. } 8 \text { S., R. } 3 \text { E., Full River County, S. Dak. Carlile } \\
\text { shale, sandstono } 43 \text { feet above shark-tooth conglomerate. }\end{array}$ \\
\hline 127 & 10281 & $\begin{array}{l}\text { E. T. Fincock, } 1919 . \text { Mulo Creek oil field, in sec. } 18, \text { T. } 39 \text { N., } \\
\text { R. } 60 \mathrm{~W} \text {., Niobrara County, Wyo. Carlile shale, in a sandstone } \\
200 \text { foct above base. }\end{array}$ \\
\hline 128 & 12691 & $\begin{array}{l}\text { W. W. Rubey, } 1924 \text {. One mile south of Newcastle, in sec. } 2, \mathrm{~T} \text {. } \\
44 \text { N., R. } 61 \text { W., Weston County, Wyo. Carlile shale, } 20 \text { feet } \\
\text { above shark-tooth conglomerate of Turner sandy member. }\end{array}$ \\
\hline 129 & 11100 & $\begin{array}{l}\text { C. R. Longwell and W. W. Rubey, } 1922 \text {. About } 2.5 \text { miles south } \\
\text { of Nowcastle, Weston County, Wyo. Carlile shale, near base. }\end{array}$ \\
\hline 130 & 21702 & $\begin{array}{l}\text { W. } \Lambda . \text { Cobban, 1948, One mile west of Newcastle, Weston } \\
\text { County, Wyo. Carlile shale, light-gray limestone concretions } \\
\text { 62. to } 70 \text { feet above base. }\end{array}$ \\
\hline
\end{tabular}

\begin{tabular}{|c|c|c|}
\hline $\begin{array}{l}\text { No. } \\
\text { on } \\
\text { fig. } \\
4\end{array}$ & $\begin{array}{l}\text { U.S.G.S. } \\
\text { Mesozoic } \\
\text { locality } \\
\text { number }\end{array}$ & $\begin{array}{l}\text { Collector, year of collection, description of } \\
\text { locality, and stratigraphic assignment }\end{array}$ \\
\hline 131 & 10334 & $\begin{array}{l}\text { Mrs. W. O. George, 1920. Near Pedro, Weston County, Wyo. } \\
\text { Carlile shale. }\end{array}$ \\
\hline 132 & 11200 & $\begin{array}{l}\text { W. W. Rubey, 1922. About a mile east of Pedro, Wyo. Carlile } \\
\text { shale. }\end{array}$ \\
\hline 133 & 10410 & $\begin{array}{l}\text { A. J.' Collier, K. C. Heald, and M. G. Gulley, 1920. Railroad cut } \\
\text { in sec. } 3 \text {, T. } 48 \text { N., R. } 66 \text { W., Weston County, Wyo. Carlile } \\
\text { shale. }\end{array}$ \\
\hline 134 & 8644 & $\begin{array}{l}\text { V. H. Barnett, } 1913 \text {. About } 15 \text { miles north of Moorcroft, in the } \\
\text { NE } 1 / 4 \mathrm{SE} 1 / 4 \text { sec. } 33 \text {, T. } 52 \text { N., R. } 67 \text { W., Crook County, Wyo. } \\
\text { Carlile shale. }\end{array}$ \\
\hline 135 & 12630 & $\begin{array}{l}\text { W. W. Rubey, 1924. Three miles 'north of Oshoto, in the NE1/4 } \\
\text { sec. } 32 \text {, T. } 54 \text { N., R. } 67 \text { W., Crook County, Wyo. Carlile shale, } \\
\text { middle of Turner sandy member. }\end{array}$ \\
\hline 136 & 21424 & $\begin{array}{l}\text { M. M. Knechtel, 1947. Eleven miles southeast of Rockypoint, } \\
\text { in sec. } 30 \text {, T. } 56 \text { N. R. } 67 \text { W., Crook County, Wyo. Carlile shale, } \\
\text { Turner sandy member. }\end{array}$ \\
\hline 137 & 21591 & $\begin{array}{l}\text { W. A. Cobban, 1948. Southwest flank of Bull Creek anticline, in } \\
\text { the NE1/4 sec. } 15 \text {, T. } 57 \text { N., R. } 62 \text { W., Crook County, Wyo. } \\
\text { Carlile shale, light-gray limestone concretions near base. }\end{array}$ \\
\hline 138 & 21798 & $\begin{array}{l}\text { W. A. Cobban, } 1941 \text {. Northeast side of Crow Creek valley, in the } \\
\text { E1/2 sec. } 9 \text {, T. } 57 \text { N., R. } 61 \text { W., Crook County, Wyo. Ferrugi- } \\
\text { nous concretions } 76 \text { to } 101 \text { feet below top of the Greenhorn } \\
\text { formation. }\end{array}$ \\
\hline 139 & 17933 & $\begin{array}{l}\text { R. P. Bryson and W. G. Pierce, 1938. Line Creek, in the } \\
\text { NW1/4NW1/4 sec. } 35 \text {, T. } 58 \text { N., R. } 103 \text { W., Park County, Wyo. } \\
\text { Cody shale. }\end{array}$ \\
\hline 140 & 17954 & $\begin{array}{l}\text { W. G. Pierce and J. B. Reeside, Jr., } 1938 \text {. Line Creek, in the } \\
\text { S.E } 1 / 4 \text { SW1 } 1 / 4 \text { sec. } 26 \text {, T. } 58 \text { N., R. } 103 \text { W., Park County, Wyo. } \\
\text { Cody shale, } 690 \text { to } 720 \text { feet above base. }\end{array}$ \\
\hline 141 & 17955 & $\begin{array}{l}\text { W. G. Pierce and J. B. Reeside, Jr., } 1938 \text {. In the SE1/4SW1/4 } \\
\text { sec. } 26, \text { T. } 58 \text { N., R. } 103 \text { W., Park County, Wyo. Cody shale, } \\
630 \text { feet above base. }\end{array}$ \\
\hline 142 & 17956 & $\begin{array}{l}\text { W. G. Pierce and J. B. Reeside, Jr., 1938. In the SW1/4SW1/4 } \\
\text { sec. } 26, \text { T. } 58 \text { N., R. } 103 \text { W., Park County, Wyo. Cody shale, } \\
590 \text { feet above base. }\end{array}$ \\
\hline 143 & 17957 & $\begin{array}{l}\text { W. G. Pierce and J. B. Reeside, Jr., 1938. Line Creek, in the } \\
\text { SE1/4SW1/4 sec. } 26, \text { T. } 58 \text { N., R. } 103 \text { W., Park County, Wyo. } \\
\text { Cody shale, } 760 \text { to } 810 \text { feet above base. }\end{array}$ \\
\hline 144 & 17958 & $\begin{array}{l}\text { W. G. Pierce, 1938. Line Creek, in the SE1/4SW1/4 sec. } 26, \text { T. } 58 \\
\text { N., T. } 103 \text { W., Park County, Wyo. Cody shale, } 1,110 \text { feet above } \\
\text { base. }\end{array}$ \\
\hline 145 & 17959 & $\begin{array}{l}\text { W. G. Pierce, } 1938 \text {. Line Creek, in the NW1/4NW1/4 sec. } 35, \mathrm{~T} \text {. } \\
58 \mathrm{~N} ., \mathrm{R} .103 \mathrm{~W} \text {., Prk County, Wyo. Cody shale, about } 700 \\
\text { feet above base. }\end{array}$ \\
\hline 146 & 18152 & $\begin{array}{l}\text { W. G. Pierce; } 1938 \text {. Line Creek, in the SE } 1 / 4 \mathrm{SW} 1 / 4 \text { sec. } 26 \text {, T. } \\
58 \text { N., R. } 103 \text { W., Park County, Wyo. Cody shale, } 1,215 \text { feet } \\
\text { above base. }\end{array}$ \\
\hline 147 & 18156 & $\begin{array}{l}\text { W. G. Pierce, 1938. In the SW1/4SW1/4 sec. } 26, \text { T. } 58 \text { N., R. } 103 \\
\text { W., Park County, Wyo. Cody shale, } 620 \text { reet above base. }\end{array}$ \\
\hline 148 & 18160 & $\begin{array}{l}\text { W. G. Pierce, } 1938 \text {. In the SW1/4SW1/4 sec. } 26 \text {, T. } 58 \text { N., R. } 103 \\
\text { W., Park County, Wyo. Cody shale, } 370 \text { feet above base. }\end{array}$ \\
\hline 149 & 17942 & $\begin{array}{l}\text { W. G. Pierce, } 1938 \text {. South of Bennett Creek, in the SW1/4SE1/4 } \\
\text { sec. } 10 \text {, T. } 57 \text { N., R. } 103 \text { W., Park County, Wyo. Cody shale, } \\
375 \text { feet above base. }\end{array}$ \\
\hline .150 & 17952 & $\begin{array}{l}\text { W. G. Pierce and J. B. Reeside, Jr., 1938. South of Bennett } \\
\text { Creek, in the SW1/4SE1/4 sec. } 10 \text {, T. } 57 \text { N., R. } 103 \text { W., Park } \\
\text { County, Wyo. Cody shale, } 375 \text { feet above base. }\end{array}$ \\
\hline 151 & 17928 & $\begin{array}{l}\text { W. G. Pierce, 1937. In the SW1/4NW1/4 sec. } 23 \text {, T. } 55 \text { N., R. } 103 \\
\text { W., Park County, Wyo. Cody shale about middle. }\end{array}$ \\
\hline 152 & 4991 & $\begin{array}{l}\text { T. E. Williard, 1907. South side of Shoshone River about } 2 \text { miles } \\
\text { east of Cody bridge, Park County, Wyo. Cody shale. }\end{array}$ \\
\hline 153 & 4960 & $\begin{array}{l}\text { C. A. Fisher and E. G. Woodruff, } 1907 \text {. Shoshone River, two } \\
\text { miles east of Cody, Wyo. Cody shale. }\end{array}$ \\
\hline 154 & 7369 & $\begin{array}{l}\text { D. F. Hewett, 1911. South side of Shoshone River } 3 \text { miles north- } \\
\text { east of Cody, Park County, Wyo. Cody shale. }\end{array}$ \\
\hline 155 & 3079 & C. A. Fisher, 1904. Near Cody, Wyoming. Cody shale. \\
\hline 156 & $\ldots$ & $\begin{array}{l}\text { Edwin Binney, Jr., } 1924 \text {. Oregon Basin, in sec. } 6, \text { T. } 51 \text { N., R. } \\
100 \text { W., Park County, Wyo. Cody shale, } 600 \text { feet above base. }\end{array}$ \\
\hline 157 & & $\begin{array}{l}\text { Edwin Binney, Jr., 1924. Oregon Basin, in sec. 6, T. } 51 \mathrm{~N} ., \mathrm{R} . \\
100 \text { W., Park County, Wyo. Cody shale, } 800 \text { feet above base. }\end{array}$ \\
\hline 158 & 17175 & $\begin{array}{l}\text { W. G. Pierce, } 1935 \text {. West flank of Fourbear anticline, half a mile } \\
\text { northwest of Palette Ranch No. 2, } 9.5 \text { miles west of Pitchfork, }\end{array}$ \\
\hline
\end{tabular}


Locaiities at which scaphite cephalopods were collected from rocks of the Colorado group-Continued

\begin{tabular}{|c|c|c|}
\hline $\begin{array}{l}\text { No. } \\
\text { on } \\
\text { fig. } \\
4\end{array}$ & $\begin{array}{l}\text { U.S.G.S. } \\
\text { Mesozoic } \\
\text { locality } \\
\text { number }\end{array}$ & $\begin{array}{l}\text { Collector, year of collection, description of } \\
\text { locality, and stratigraphic assignment }\end{array}$ \\
\hline 159 & 17176 & $\begin{array}{l}\text { D. A. Andrews and W. G. Pierce, } 1935 . \text { Eight miles west-north- } \\
\text { west of Pitchfork, in the NW1/4 sec. } 21, \text { T. } 48 \text { N., R. } 103 \text { W., } \\
\text { Park County, Wyo. Cody shale, } 200 \text { feet above base. }\end{array}$ \\
\hline 160 & 17168 & $\begin{array}{l}\text { D. A. Andrews, } 1935 \text {. South side of Spring Creek, near Meeteetse, } \\
\text { Wyo. Cody shale. }\end{array}$ \\
\hline 161 & 17164 & $\begin{array}{l}\text { W. G. Pierce, 1935. Pitchfork anticline, in the SE1/4SE1/4 sec. } \\
2 \text {, T. } 48 \text { N., R. } 102 \text { W., Park County, Wyo. Cody shale, } 1,400 \\
\text { feet above base. }\end{array}$ \\
\hline 162 & 17110 & $\begin{array}{l}\text { W. G. Pierce, 1935. In the NE1/4NW1/4 sec. } 24, \text { T. } 48 \text { N., R. } 102 \\
\text { W., Park County, Wyo. Cody shale. }\end{array}$ \\
\hline 163 & 17115 & $\begin{array}{l}\text { W. G. Pierce, } 1935 \text {. In the NE } 1 / 4 \text { sec. } 10, \text { T. } 46 \text { N., R. } 101 \text { W., Park } \\
\text { County, Wyo. Cody shale. }\end{array}$ \\
\hline 164 & 10422 & $\begin{array}{l}\text { M. G. Gulley and K. C. Heald, } 1920 \text {. South of Kirby Creek, } \\
\text { in the NE } 1 / 4 \text { sec. } 20 \text {, T. } 43 \text { N., R. } 92 \text { W., Hot Springs County, } \\
\text { Wyo. Cody shale. }\end{array}$ \\
\hline 165 & 10421 & $\begin{array}{l}\text { M. G. Gulley and K. C. Heald, } 1920 \text {. South Branch of Kirby } \\
\text { Creek, about } 15 \text { miles east of Lucerne, in sec. } 22, \text { T. } 43 \text { N., R. } \\
92 \text { W., Hot Springs County, Wyo. Cody shale. }\end{array}$ \\
\hline 166 & 8915 & $\begin{array}{l}\text { W. P. Woodring, 1914. Nine miles northwest of Nowood, in the } \\
\text { NW1/4NE1/4 sec. } 30, \text { T. } 43 \text { N., R. } 89 \text { W., Washakie County, Wyo. } \\
\text { Cody shale. }\end{array}$ \\
\hline 167 & 4538 & $\begin{array}{l}\text { M. A. Pishel, 1907. Four miles southeast of Greybull, in the } \\
\text { NW1/4SW1 } 1 / 4 \text { sec. } 25, \text { T. } 52 \text { N., R. } 93 \text { W., Big Horn County, Wyo. } \\
\text { Cody shale. }\end{array}$ \\
\hline 168 & 4958 & $\begin{array}{l}\text { M. A. Pishel, 1907. Four miles northeast of Basin, in the } \\
\text { SW } 1 / 4 \text { NE } 1 / 4 \text { sec. } 30 \text {, T. } 52 \text { N., R. } 92 \text { W., Big Horn County, Wyo. } \\
\text { Cody shale. }\end{array}$ \\
\hline 169 & 8278 & $\begin{array}{l}\text { J. Henderson, 1913. Twelve miles east of Basin, Big Horn County, } \\
\text { Wyo. Cody shale. }\end{array}$ \\
\hline 170 & 9615 & $\begin{array}{l}\text { C. J. Hares, } 1916 \text {. Garland anticline, one mile west of Byron, in } \\
\text { sec. } 33 \text {, T. } 56 \text { N., R. } 97 \text { W., Big Horn County, Wyo. Cody } \\
\text { shale. }\end{array}$ \\
\hline 171 & 4539 & $\begin{array}{l}\text { C. W. Washburne, 1907. Garland coal field, Park County, Wyo. } \\
\text { Cody shale. }\end{array}$ \\
\hline 172 & 9770 & $\begin{array}{l}\text { C. J. Hares, 1916. Eight miles southeast of Lovell, Big Horn } \\
\text { County, Wyo. Cody.shale, lower part. }\end{array}$ \\
\hline 173 & 4540 & $\begin{array}{l}\text { H. P. Little, 1907. Two miles east of Cowley, Big Horn County, } \\
\text { Wyo. Cody shale. }\end{array}$ \\
\hline 174 & 9675 & $\begin{array}{l}\text { C. J. Hares, } 1916 \text {. Southeast of Frannie, in sec. } 23, \text { T. } 57 \text { N., R. } \\
97 \text { W:, Big Horn County, Wyo. Cody shale. }\end{array}$ \\
\hline 175 & 9669 & $\begin{array}{l}\text { C. J. Hares, } 1916 . \text { About } 1.5 \text { miles south of Frannie, in sec. } 24 \text {, } \\
\text { T. } 58 \text { N., R. } 98 \text { W., Park County, Wyo. Cody shale. }\end{array}$ \\
\hline 176 & 20701 & $\begin{array}{l}\text { J. D. Love, 1945. Gros Ventre area. Teton County, Wyo. } \\
\text { Frontier formation. }\end{array}$ \\
\hline 177 & 21109 & $\begin{array}{l}\text { H. R. Bergquist, D. C. Duncan; R. K. Hose, and J. D. Love, } \\
\text { 1947. In the NE1/4NW1/4 sec. } 20, \text { T. } 42 \text { N., R. } 112 \text { W:, Teton } \\
\text { County, Wyo. Cody shale, } 500 \text { feet above base. }\end{array}$ \\
\hline 178 & 21173 & $\begin{array}{l}\text { D. C. Duncan, R. K. Hose, and J. D. Love, } 1947 \text {. Same locality } \\
\text { as } 21109 \text {. Cody shale, sandstone unit } 607 \text { to } 614 \text { feet above base. }\end{array}$ \\
\hline 179 & 21110 & $\begin{array}{l}\text { H. R. Bergquist, } 1917 \text {. One mile northwest of Upper Slide Lake, } \\
\text { in the NW1/4NE1/4 sec. } 19 \text {, T. } 42 \text { N., R. } 112 \text { W., Teton County, } \\
\text { Wyo. Cody shale, } 1,283 \text { feet above base. }\end{array}$ \\
\hline 180 & 21179 & $\begin{array}{l}\text { H. R. Berquist, D. C. Duncan, R. K. Hose, and J. D. Love, } 1947 . \\
\text { North side of bend on Bacon Creek north of Bar Y Creek, in } \\
\text { the NE1/4NE1/4 sec. 22, T. } 41 \text { N., T. 111. W., Teton County, } \\
\text { Wyo. Frontier formation, } 180 \text { feet below top. }\end{array}$ \\
\hline 181 & 17301 & $\begin{array}{l}\text { W. W. Rubey, 1936. South Piney Creek, in the NE1/4NW1/4 } \\
\text { sec. 15, T. } 29 \text { N., R. } 115 \text { W., Sublette County, Wyo. Frontier } \\
\text { formation. }\end{array}$ \\
\hline 182 & 17302 & $\begin{array}{l}\text { W. W. Rubey, 1936. In the NW1/4 sec. } 22, \mathrm{~T} .29 \mathrm{~N} ., \mathrm{R} .115 \mathrm{~W} . \text {, } \\
\text { Sublette County, Wyo. Frontier formation. }\end{array}$ \\
\hline 183 & 17315 & $\begin{array}{l}\text { W. W. Rubey, 1936. Afton quadrangle, in the NW1/4NW1/4 sec. } \\
9, \text { T. } 26 \text { N., R. } 113 \text { W., Lincoln County, Wyo. Hilliard shale. }\end{array}$ \\
\hline 184 & 6286 & $\begin{array}{l}\text { Robert Forrester, } 1909 . \text { Glencoe, Lincoln County, Wyo. Fron- } \\
\text { tier formation, just above Kemmerer coal bed. }\end{array}$ \\
\hline 185 & 19532 & $\begin{array}{l}\text { J. D. Love, 1944. East Fork of Sheep Creek, } 35 \text { miles north of } \\
\text { Riverton, in sec. } 15 \text {, T. } 6 \text { N., R. } 2 \text { E., Fremont County, Wyo. } \\
\text { Frontier formation, top sandstone unit. }\end{array}$ \\
\hline 186 & 20920 & $\begin{array}{l}\text { H. H. R. Sharkey, 1945. Bargee area, in the NE1/4NE1/4 sec. } 27 \text {, } \\
\text { T. } 7 \text { N., R. } 1 \text { W., Fremont County, Wyo. Cody shale, about } \\
1,000 \text { feet above base. }\end{array}$ \\
\hline
\end{tabular}

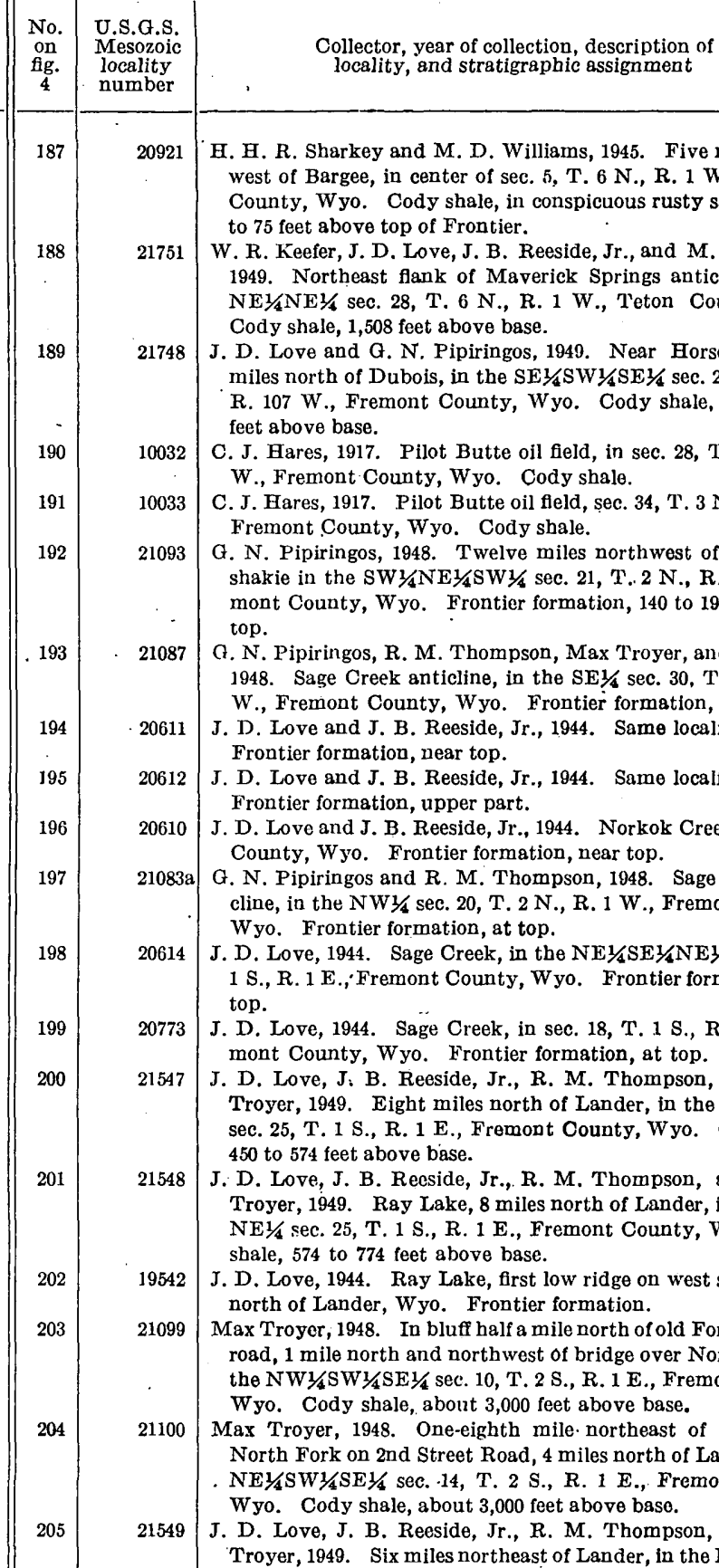
198

2

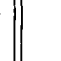
19

.

$$
\text { , }
$$

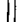

20 .

\section{1}

$$
2
$$

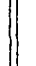

20 
Localities at which scaphite cephalopods were collected from rocks of the Colorado group-Continued

\begin{tabular}{|c|c|c|}
\hline $\begin{array}{l}\text { No. } \\
\text { on } \\
\text { fig. } \\
4\end{array}$ & $\begin{array}{l}\text { U.S.G.S. } \\
\text { Mesozoic } \\
\text { Incality } \\
\text { number }\end{array}$ & $\begin{array}{l}\text { Collector, yoar of collection, description of } \\
\text { locality, and stratigraphic assignment }\end{array}$ \\
\hline 211 & 18946 & $\begin{array}{l}\text { H. F. R. Sharkey, 1944. Dallas dome, in sec. } 16, \text { T. } 32 \text { N., R. } 98 \\
\text { W., Fremont County, Wyo. Frontier formation. }\end{array}$ \\
\hline 212 & 18941 & $\begin{array}{l}\text { F. A. Tourtelot, } 1944 \text {. Dorby dome, in sec. } 26, \text { T. } 32 \text { N., R. } 98 \\
\text { W., Fremont County, Wyo. Frontier formation. }\end{array}$ \\
\hline 213 & 8984 & $\begin{array}{l}\text { C. I. Fares, 1914. A bout } 30 \text { miles east of Lander, Fremont } \\
\text { County, Wyo. Frontier formation. }\end{array}$ \\
\hline 214 & 8985 & $\begin{array}{l}\text { C. J. Fares, 1914. A bout } 30 \text { miles east of Lander, Fremont } \\
\text { County, Wyo. Frontier formation, } 50 \text { feet lower than Loc. } 8984 \text {. }\end{array}$ \\
\hline 215 & 21538 & $\begin{array}{l}\text { C. N. Pipiringos and } \mathrm{K} \text {. Yenne, 1949. Conant Creek, } 35 \text { miles } \\
\text { orst of Lander, in the NW1/4SW1/4 sec. 13, T. } 33 \mathrm{~N} \text {., R. } 94 \mathrm{~W} \text {., } \\
\text { Fremont County, Wyo. Frontier formation, } 53 \text { feet below top. }\end{array}$ \\
\hline 216 & 21757 & $\begin{array}{l}\text { C. N. PIpiringos, } 1948 \text {. Same locality as } 21538 \text {. Frontier forma- } \\
\text { tion, near top. }\end{array}$ \\
\hline 217 & 20613 & $\begin{array}{l}\text { J. D. Love, 1944. Conant Creek, Fremont County, W yo. Frontier } \\
\text { formation. }\end{array}$ \\
\hline 218 & 9008 & $\begin{array}{l}\text { J. B. Reoside, Jr., 1914. Muskrat Creek, T. } 32 \text { N., R. } 91 \text { W., } \\
\text { Fromont County, Wyo. Cody shale. }\end{array}$ \\
\hline 210 & 21102 & $\begin{array}{l}\text { R. M. Thompson, } 1948 \text {. Ten miles southwest of Raderville, in } \\
\text { the NW1/s sec. } 12, T \text {. } 33 \text { N., R. } 90 \text { W., Fremont County, Wyo, } \\
\text { Cody shale, } 50 \text { to } 100 \text { feet above top sandstone of Frontier. }\end{array}$ \\
\hline 220 & 8524 & $\begin{array}{l}\text { C. J. Fares, 1913: Scc. 9, T. } 32 \text { N., R. } 86 \text { W., Natrona County, } \\
\text { Wyo. Cody shale. }\end{array}$ \\
\hline 221 & 8511 & $\begin{array}{l}\text { C. J. Hares, 1013. Twenty-seven miles northwest of Casper, in } \\
\text { sec. } 36, \text { T. } 37 \text { N., R. } 82 \text { W., Natrona County, Wyo. Frontier } \\
\text { formation. }\end{array}$ \\
\hline 222 & 4448 & $\begin{array}{l}\text { C. T. Tupton, } 1907 . \text { About } 4.5 \text { miles southeast of Big Muddy } \\
\text { station, in T. } 33 \text { N., R. } 76 \text { W., Converse County, wyo. }\end{array}$ \\
\hline 223 & 20917 & $\begin{array}{l}\text { J. D. Love, 1046. Eleven miles south of Douglas, in the NW1/4 } \\
\text { NWb/4 sec. } 5, \mathrm{~T} .30 \mathrm{~N} \text {., R. } 71 \text { W., Converse County, Wyo. } \\
\text { Frontier formation. }\end{array}$ \\
\hline 224 & 4872 & $\begin{array}{l}\text { E. B. Hopkins, } 1307 \text {. Six miles east of Crooks Crap oil ficld, in } \\
\text { the NWY sec. } 19, \text { T. } 28 \text { N., R. } 91 \text { W., Fremont County, Wyo. }\end{array}$ \\
\hline 225 & 9020 & $\begin{array}{l}\text { C. J. Hares and J. B. Reeside, Jr., 1914. One mile east of Jack } \\
\text { Crelvo's Ranch near Whiskey Peak, in sec. } 26 \text {, T. } 28 \text { N., R. } 90 \\
\text { W., Fromont County, Wyo. Cody shale. }\end{array}$ \\
\hline 226 & 10464 & $\begin{array}{l}\text { N. W. Baso, } 1920 \text {. Lost Soldier oil fleld, Sweetwater County, } \\
\text { Wyo. Frontier formation, shale bencath top sandstone. }\end{array}$ \\
\hline 227 & 10456 & $\begin{array}{l}\text { A. R. Fath and C. Y. Fisieh, } 1920 \text {. Thirty-two miles north- } \\
\text { northwest of lRawlins, in sec. 7, T. } 26 \text { N., R. } 88 \text { W., Carbon } \\
\text { County, Wyo. Stecle shale, 1,728 feet above base. }\end{array}$ \\
\hline 228 & 4060 & $\begin{array}{l}\text { A. C. Veatch, } 1906 \text {. Three miles southeast of Rawlins, in the } \\
\text { NE1/4 sec. } 27 \text {, T. } 21 \text { N., R. } 87 \text { W., Carbon County, Wyo. Fron- } \\
\text { tier formation, } 570 \text { fect above Dakota sandstone. }\end{array}$ \\
\hline 220 & 4060 & $\begin{array}{l}\text { A. C. Voatch, } 1906 \text {. Three milos southeast of Rawlins, in the } \\
\text { NW1/4 sec. } 26, T .21 \text { N., R. } 87 \text { W., Carbon County, Wyo. Fron- } \\
\text { tior formation. }\end{array}$ \\
\hline 230 & 3712 & $\begin{array}{l}\text { A. C. Voatch, } 1906 \text {. Fifteen miles south-southwest of Hanna, in } \\
\text { the SIE } 1 / 4 \text { NE1/4 sec. } 30 \text {, T. } 20 \text { N., R. } 82 \text { W., Carbon County, Wyo. } \\
\text { Frontier formation, } 680 \text { feet above Dakota sandstone. }\end{array}$ \\
\hline 231 & 22125 & $\begin{array}{l}\text { F. V. Flaydon, } 1870 \text {. Medicine Bow River, southeastern Wyo. } \\
\text { (Frontier formation.) }\end{array}$ \\
\hline 232 & 530 & $\begin{array}{l}\text { A. Hyatt, and Russell, 1888. Railroad cut half a mile east of } \\
\text { Aurora, Carbon County, Wyo. }\end{array}$ \\
\hline 233 & 533 & $\begin{array}{l}\text { A. Hyatt, and Russell, } 1888 \text {. Railroad cut } 1 \text { mile north of Aurora, } \\
\text { Carbon County, Wyo. }\end{array}$ \\
\hline 234 & 399 & $\begin{array}{l}\text { L. F. Ward. One mile north of Rock Creek, in sec. } 30 \text {, T. } 22 \text { N., } \\
\text { R. } 75 \text { W., Albany County, Wyo. Carlile shale. }\end{array}$ \\
\hline 235 & 6270 & $\begin{array}{l}\text { Robort Forrester, } 1909 . \text { Four miles east of Oak Springs, Sevier } \\
\text { County, Utah, Mancos shale. }\end{array}$ \\
\hline 236 & 12583 & $\begin{array}{l}\text { E. M. Spicker, 1924. Near Emery, Emery County, Utah. Man- } \\
\text { cos shale, Forron sandstone member. }\end{array}$ \\
\hline 237 & 22120 & $\begin{array}{l}\text { Philip Katich, 1849. Six mlles east of Clawson, in sec. } 26 \text {, T. } 19 \mathrm{~S} . \text {, } \\
\text { R. } 8 \text { F., Emcry County, Utah. Blue Gate shale, near base. }\end{array}$ \\
\hline 238 & 6945 & $\begin{array}{l}\text { Robert Forrester, 1910. Irrigated Lands Company tunnel be- } \\
\text { tween Price and Spring Glen, Carbon County, Utah. Mancos } \\
\text { shale. }\end{array}$ \\
\hline 239 & 11056 & $\begin{array}{l}\text { J. B. Reoside, Jr., and E. M. Spieker, 1923. } 300 \text { feet north of Car- } \\
\text { bon County High School, Price, Utah. Mancos shale. }\end{array}$ \\
\hline 240 & 13310 & H. F. Moses, 1925. Three miles north of Woodside, in the SE1/4 \\
\hline & . & $\begin{array}{l}\text { SW1/4 soc. } 28, \text { T. } 17 \text { S., R. } 14 \text { E., Emery County, Utah. Mancos } \\
\text { shale. }\end{array}$ \\
\hline 241 & 834 & $\begin{array}{l}\text { T. W. Stanton, 1802. Four miles northwest of Woodside, Emery } \\
\text { County, Utah. Mancos shale. }\end{array}$ \\
\hline
\end{tabular}

\begin{tabular}{|c|c|c|}
\hline $\begin{array}{c}\text { No. } \\
\text { on } \\
\text { flg. } \\
4\end{array}$ & $\begin{array}{l}\text { U.S.G.S. } \\
\text { Mesozoic } \\
\text { locality } \\
\text { number }\end{array}$ & $\begin{array}{l}\text { Collector, year of collection, description of } \\
\text { locality, and stratigraphic assignment }\end{array}$ \\
\hline
\end{tabular}

$24213240 \quad \begin{aligned} & \text { J. B. Reeside, Jr., and E. M. Spieker, 1925. One mile west of } \\ & \text { Desert, Emery County, Utah. Mancos shale, } 20 \text { feet above }\end{aligned}$
Desert, Emery County, Utah. Mancos shale, 20 feet above Ferron sandstone member.

13243 J. B. Reeside, Jr., and E. M. Spieker, 1925. One mile east of Desert, Emery County; Utah. Mancos shale, 970 feet above base.

13261 J. B. Reeside, Jr., 1925: Near Desert, Emery County, Utah. Mancos shale, 1,250 feet above base.

13262 J. B. Reeside, Jr., 1925. Near Desert, Emery County, Utah. Mancos shale, 1,340 feet above base.

13263 J. B. Reeside, Jr., 1925. Near Desert, Emery County, Utah. Mancos shale, 1,400 feet above base.

13331 J. D.' Fisher, 1925. Sec. 3, T. 20 S., R. 16 E., Emery County, Utah. Mancos shale.

21426 W. A. Cobban, 1944. Brush Creek, 9.5 miles northeast of Vernal, in center of sec. 11, T. $3 \mathrm{~S}$., R $22 \mathrm{E}$., Uintah County, Utah. Mancos shale, 180 fect above top of Frontier sandstone member.

21427 W. A. Cobban, 1945. Northeast side of Ashley Creek, 2.5 miles west of Jensen, in the SW $1 / 4 \mathrm{SE} 1 / 4$ sec. 24 , T. 5 S., R. 22 E., Uintah County, Utah. Mancos shale, about 800 feet above base.

4363 H. S. Gale, 1907. Eleven miles north of Rangely, in sec. 12, T. 3 N., R. 102 W., Moffat County, Colo. Mancos shale.

21764 W. A. Cobban, 1945. Three miles west of Skull Creek, in the SE $1 / 4$ SE $1 / 4$ sec. 4,-T. 3 N., R. 101 W., Moffat County, Colo. Mancos shale, top of Frontier sandstone member.

11670 W. H. Bradley, J. B. Reeside, Jr., and J. D. Sears, 1923. Vermilion Creek, in the SW1/4NW1/4 sec. 11, T. 10 N., R. $101 \mathrm{~W}$, Moffat County, Colo. Mancos shale, 20 feet above top of Frontier sandstone member.

11678 W. H. Bradley, J. B. Reeside, Jr., and J. D. Sears, 1923. Vermilion Creek, in the NE1/4NW1/4 sec. 11, T. 10 N., R. 101 W., Moffat County, Colo. Mancos shale, 1,002 feet above base.

11685 W. H. Bradley, J. B. Reeside, Jr., and J. D. Sears, 1923. Vermilion Creek, in the SE1/4SW1/4 sec. 2, T. 10 N., R. 101 W., Moffat County, Colo. Mancos shale, 1,222 feet above base.

11699 W. H. Bradley, J. B. Reeside, Jr., and J. D. Sears, 1923. Vermillion Creek, in the NW1/4 NE1/4 sec. 11, T. 10 N., R. 101 W., Moffat County, Colo. Mancos shale, 1,642 feet above base.

11705 W. H. Bradley, J. B. Reeside, Jr., and J. D. Sears, 1923. Same locality as 11699 . Mancos shale, 1,767 feet above base.

13699 J. B. Reeside, Jr., 1926. Fourteen miles northwest of Craig, in the SW1/4SE1/4SE1/4 sec. 27, T. 9 N., R: 29 W., Moffat County. Colo. Mancos shale.

6252 A. S. Beekly, 1909. Edgerton Creek Gap, 10 miles south of Glenwood Springs, in sec. 1, T. 8 S., R. 89 W., Garfeld County, Colo. Mancos shale.

6950 C. W. Washburne, 1910. At Cedar, San Miguel County, Colo Mancos shale.

2007 C. W. Purington, 1896. Near Rico, Colorado. Mancos shale within 100 feet of Dakota sandstone.

7389 M. A. Pishel and H. F. Wright, 1911, Twelve miles south west of Cortez, in sec. 3, 'T. 34 N., R. 17 W., Montezuma County; Colo.

17431 J. D. Northrop, 1934. Center of NW1/4 sec. 21, T. 36 N., R. 14 W., Montezuma County, Colo. Mancos shale, 475 feet above base.

4012 C. D. Smith, 1906. North side of Mancos-Thompson Park road, one mile southeast of Menefee ranch house, Durango coal fleld, Colo. Mancos shalc, in limestone ledges.

$10506 \mathrm{~J}$. B. Reeside, Jr., 1920. A mile and a half west of Durango, in the NW1/4SE1/4 sec. 18, T. 35 N., R. 9 W., La Plata County, Colo. Mancos shale, about 300 feet above Dakota sandstone.

7360 C. W. Cooke, 1911. At base of mountain a quarter of a mile north of head of Deadman's Creek, 10 miles southwest of Castle Rock, Douglas County, Colo. Benton shale.

14305 A. E. Brainerd and I. A. Keyte, 1928. Sixteen miles east of Trinidad, in sec. 1, T. 32 S., R. 62 W., Las Animas County, Colo. Apishapa shale, near top.

A. L. Morrow. Three miles south-southeast of Tipton, in the SE $1 / 4$ sec. 4, 'T. 9 S., R. 10 W., Mitchell County, Kans. Carlile shale, in upper part of Blue Hill shale member.

21838 W. A. Cobban, 1948. Three miles south-southeast of Tipton, in the SE $1 / 4$ sec. 4, T. 9 S., R. 10 W., Mitchell County, Kans. Carlile shale, in upper part of Blue Hill shale member. 
SCAPHITOID CEPHALOPODS OF THE COLORADO GROUP

Localities at which scaphite cephalopods were collected from rocks of the Colorado group-Continued

\begin{tabular}{|c|c|c|c|c|c|}
\hline $\begin{array}{l}\text { No. } \\
\text { on } \\
\text { fig. } \\
4\end{array}$ & $\begin{array}{l}\text { U.S.G.S. } \\
\text { Mesozoic } \\
\text { locality } \\
\text { number }\end{array}$ & $\begin{array}{l}\text { Collector, year of collection, description of } \\
\text { locality, and stratigraphic assignment }\end{array}$ & $\begin{array}{l}\text { No. } \\
\text { on } \\
\text { fig. } \\
4\end{array}$ & $\begin{array}{l}\text { U.S.G.S. } \\
\text { Mesozoic } \\
\text { locality } \\
\text { number }\end{array}$ & $\begin{array}{l}\text { Collector, year of collection, description of } \\
\text { locality, and stratigraphic assignment }\end{array}$ \\
\hline 269 & 11591 & $\begin{array}{l}\text { W. B. Emery, 1923. Top of the "Black Hill" on Shiprock- } \\
\text { Biltabito road about } 14 \text { miles west of Shiprock Agency, Navajo } \\
\text { Indian Reservation, San Juan County, N. Mex. Mancos shale, } \\
150 \text { feet below base of Tocito sandstone lentil. }\end{array}$ & 282 & $\begin{array}{r}17633 \\
17635\end{array}$ & $\begin{array}{l}\text { R. P. Bryson, 1937. About } 7.5 \text { miles north-northeast of El Vado } \\
\text { Reservoir, Rio Arriba County, N. Mex. Mancos shale. } \\
\text { R. P. Bryson, 1937. Near El Vado, Rio Arriba County, N. Mex. } \\
\text { Mancos shale. }\end{array}$ \\
\hline 270 & 18874 & $\begin{array}{l}\text { N. W. Bass, } 1943 \text {. On Biltabito road } 15 \text { miles west of Shiprock } \\
\text { bridge, San Juan County, N. Mex. Mancos shale, in sandstone }\end{array}$ & 283 & 17637 & $\begin{array}{l}\text { R. P. Bryson, 1937. Upper Lagunas Creek, Rio Arriba County, } \\
\text { N. Mex. Mancos shale. }\end{array}$ \\
\hline 271 & 12008 & $\begin{array}{l}\text { J. B. Reeside, Jr., } 1923 \text {. Center of the NW1/4SW1/4 sec. } 25, \text { T. } 30 \\
\text { N., R. } 17 \text { W., San Juan County, N. Mex. Mancos shale, } 698 \\
\text { feet below base of Mesaverde formation. }\end{array}$ & 284 & 16784 & $\begin{array}{l}\text { C. H. Dane, 1933. East of Monero and north of Denver Rio } \\
\text { Grande Western R. R., in northeast part of T. } 31 \mathrm{~N} \text {., R. } 1 \text { E., } \\
\text { Rio Arriba County, N. Mex. Mancos shale, 1,000. feet below } \\
\text { base of upper part of the Hosta sandstone member of the Mesa- }\end{array}$ \\
\hline & & $\begin{array}{l}\text { W., San Juan County, N. Mex. Mancos shale, } 115 \text { feet below } \\
\text { top. }\end{array}$ & 285 & 17638 & $\begin{array}{l}\text { C. H.Dane,1937. Near Chama-Monero road, Rio Arriba County, } \\
\text { N. Mex. Mancos shale. }\end{array}$ \\
\hline 273 & 10368 & $\begin{array}{l}\text { W. E. Brysant; C. E. Dobbin, and J. B. Reeside, Jr., 1920. Four- } \\
\text { teen miles southwest from Shiprock Mtn. (Wilson Peak), in } \\
\text { sec. } 5 \text { or } 8, \text { T. } 9 \text { N., R.4 W., San Juan County, N. Mex. Mancos }\end{array}$ & 286 & $\ldots$ & $\begin{array}{l}\text { W. S. Pike, Jr., 1931. West side of Cebolleta Mesa, in sec. 17, T. } 8 \\
\text { N., R. } 9 \text { W., Valencia County, N. Mex. Mancos shale, } 275 \\
\text { feet below top. }\end{array}$ \\
\hline 274 & 11593 & $\begin{array}{l}\text { shale. } \\
\text { W. B. Emery, 1923. Two miles west of Tsenosti Trading Post, } \\
\text { Navajo Indian Reservation, San Juan County, N. Mex. Mancos }\end{array}$ & 287 & 15590 & $\begin{array}{l}\text { C. B. Hunt, 1930. Six miles east of Seboyeta, Valencia County, } \\
\text { N. Mex. Mancos shale, } 679 \text { feet above base, and } 250 \text { feet below } \\
\text { base of Gallup sandstone member of Mesaverde formation. }\end{array}$ \\
\hline & & $\begin{array}{l}\text { shale, from thin sandstone about } 150 \text { feet below base of Tocito } \\
\text { sandstone lentil. }\end{array}$ & 288 & 15600 & $\begin{array}{l}\text { C. B. Hunt, 1930. About 5.5 miles north of Evan's Park, Cebolleta } \\
\text { Grant, Sandoval County, N. Mex. Mancos shale, } 200 \text { feet }\end{array}$ \\
\hline 275 & 11594 & $\begin{array}{l}\text { W. B. Emery, 1923. Two miles west of Tsenosti Trading Post, } \\
\text { Navajo Indian Reservation, San Juan County, N. Mex. Man- } \\
\text { cos shale, in banded calcareous concretions } 120 \text { feet below Tocito } \\
\text { sandstone lentil. }\end{array}$ & 289 & 15601 & $\begin{array}{l}\text { below Gallup sandstone member of the Mesaverde formation. } \\
\text { C. B. Hunt, 1930. About } 2 . \dot{5} \text { miles north-northeast of Evan's } \\
\text { Ranch, Nuestra Senora de la Luz de las Lagunitas Grant, } \\
\text { Sandoval County, N. Mex. Mancos shale, } 400 \text { feet below }\end{array}$ \\
\hline 276 & 15580 & $\begin{array}{l}\text { C. B. Hunt, } 1930 \text {. Sixteen miles northwest of San Mateo, in the } \\
\text { NE1/4 sec. } 2, T .14 \mathrm{~N} \text {., R. } 10 \mathrm{~W} \text {., McKinley County, N. Mex. } \\
\text { Mancos shale, } 200 \text { feet below base of Gallup sandstone member } \\
\text { of Mesaverde formation. }\end{array}$ & 290 & 16115 & $\begin{array}{l}\text { Gallup sandstone member of the Mesaverde formation. } \\
\text { C. B. Hunt, } 1931 \text {. Nineteen miles southeast of Dominguez, in } \\
\text { the SW1/4NW1/4 sec. 5, T. } 14 \text { N., R. } 1 \text { E., Sandoval County, } \\
\text { N. Mex. Mancos shale, top of Carlile shale member. }\end{array}$ \\
\hline 277 & 13283 & $\begin{array}{l}\text { B. C. Renick,1925. Senorita Canyon, Sandoval County, N. Mex. } \\
\text { Mancos shale. }\end{array}$ & 2 & 7180 & $\begin{array}{l}\text { W. T. Lee, 1911. Omera mine, east flank of Ortiz Mountains, } \\
\text { Santa Fe County, N. Mex. Mancos shale, uppermost.part. }\end{array}$ \\
\hline 278 & 16818 & $\begin{array}{l}\text { C. B. Hunt, 1933. Three miles west of Gallina, in the SW1/4NW1/4 } \\
\text { sec. } 13, \text { T. } 23 \text { N., R. } 1 \text { W., Rio Arriba County, N. Mex. Mancos }\end{array}$ & 29 & 716 & $\begin{array}{l}\text { W. T. Lee, 1911. One mile southwest of Waldo, Santa Fe County, } \\
\text { N. Mex. Mancos shale, upper } 200 \text { feet, and Mesaverde forma- } \\
\text { tion, basal part. }\end{array}$ \\
\hline 279 & 515 & $\begin{array}{l}\text { shale. } \\
\text { J. W. Powell, 1887. Near Lagoon, } 5 \text { miles from Gallina, Rio } \\
\text { Arriba County, N. Mex. Mancos shale. }\end{array}$ & 293 & 3532 & $\begin{array}{l}\text { W. T. Lee, } 1905 \text {. One mile north of Galisteo Creek and } 1 \text { mile } \\
\text { east of head of Canyon del Yeso, Santa Fe County, N. Mex. } \\
\text { Mancos shale, uppermost part. }\end{array}$ \\
\hline 280 & 17628 & $\begin{array}{l}\text { R. P. Bryson, 1937. About } 7.5 \text { miles north-northeast of north end } \\
\text { of El Vado Reservoir, Rio Arriba County, N. Mex. }\end{array}$ & 294 & 5596 & $\begin{array}{l}\text { W. T. Lee, 1908. Outlet of Johnson Park, Raton coal fleld, north- } \\
\text { eastern Colfax County. N. Mex. Niobrara formation. }\end{array}$ \\
\hline
\end{tabular}

\section{SYSTEMATIC DESCRIPTIONS}

Scaphites delicatulus Warren var. greenhornensis Cobban, n. var.

\section{Plate 1, figures $1-3$}

This variety is represented in the collections by only two individuals, the smaller of which has been selected as the type. It is $28 \mathrm{~mm}$. long, $23 \mathrm{~mm}$. high, and 14.2 $\mathrm{mm}$. wide, and has about 85 ventral ribs on the exposed whorls of which 40 are on the living chamber. Primary ribs number 15 on the living chamber and about 16 or 17 on the last septate whorl. Neither the internal whorls nor the suture were seen.

The variety differs from the holotype of Scaphites delicatulus Warren (1930, p. 66, pl. 3, fig. 3; pl. 4, figs. 7,8$)$ in its coarse ribbing near the aperture and greater number of primaries in proportion to the number of secondaries on the living chamber, the straight part having two or three secondaries to each primary whereas Warren's specimen shows four to six secondaries to each primary. The tubercles are not as conspicuous or pointed as on 'Warren's type. The variety more closely resembles a specimen of $S$. delicatulus more recently figured by Warren (1947, p. 123, pl. 29, fig. 5), differing from it chiefly in the presence of coarse ribbing near the aperture and lack of pointed tubercles.

Scaphites brittonensis Moreman (1942, p. 215, pl. 34, figs. 1, 2; text fig. 2) from the Britton formation of Texas is related to the variety greenhornensis but differs by its smaller size, pointed tubercles, and uniform spacing of the ventral ribs over the entire living chamber. Of the European species $S . d$.greenhornensis closely resembles $S$ : aequalis Sowerby although that species may lack terminal coarse ribbing. One of the specimens figured as $S$. geinitzi d'Orbigny by Geinitz (1874, p. 191 , pl. 35 , fig. 3 ) is coarsely ribbed at the end of the living chamber and appears to differ from the Greenhorn form only in its high, rounded tubercles.

Holotype, U.S.N.M. 106766.

The holotype is from a calcareous concretion about 10 feet below the top of the Greenhorn formation on the north flank of the Black Hills uplift, in the W $1 / 2$ sec. 14, T. 9 S., R. 60 E., Carter County, Mont. The 
external mold of part of a large specimen was found in the living chamber of a Vascoceras thomi Reeside in the Greenhorn calcareous member of the Cody shale on the Crow Indian Reservation in south-central Montana.

\section{Scaphites larvaeformis Meek and Hayden}

Plate 1, figures 4-15

1859. Scaphites larvaeformis Meek and Hayden, Acad. Nat. Sci. Philadelphia Proc., vol. 1.0, p. 58.

1876. Scaphites larvaeformis Meek and Hayden. Meek, U. S. Geol. Survey Terr. Rept., vol. 9, p. 418, pl. 6, figs. 6a-c. 1893. Scaphites larvaeformis Meek and Hayden. Stanton, U. S. Geol. Survey Bull. 106, p. 182, pl. 44, fig. 2.

1898. Scaphites larvaeformis Meek and Hayden. Logan, Kansas Univ. Geol. Survey, vol. 4, p. 473, pl. 104, fig. 2.

1915. Scaphites larvaeformis Meek [and Hayden]. Frech, Centralbl. Mineralogie, Jahrg. 1915, no. 21, p. 556, fig. 1.

1916. Holcoscaphites larvaeformis (Meek and Hayden). Nowak, K.-k. Geol. Reichsanstalt Verh., Jahrg. 1916, no. 3, p. 66 .

1927. Scaphites larvaeformis Meek and Hayden. Reeside, U. S. Geol. Survey Prof. Paper 150-B, p. 31.

The description by Meek (1876, p. 418)- is as follows:

Shell small, transversely. subovate, compressed, evenly rounded on the periphery; volutions slender, nearly round, the inner or coiled ones forming only a very small part of the entire shell, and so closely involuted as to leave only a very small umbilical pit; extended body portion rather long, slender, and straight to the recurvature, thence continued backward until it comes nearly in contact with coiled inner volutions; aperture apparently circular; surface ornamented by small costae, which pass from the inner side of the volutions to about half way across their lateral surfaces, where they swell into small, obscure, transversely elongated nodes, and then branch each into two or three smaller linear ribs, all of which pass straight over the periphery.

Length, $0.87 \mathrm{inch}$; height, $0.63 \mathrm{inch}$; convexity, 0.33 inch.

The septa of this species are comparatively rather simple, being each provided with but two principal lateral lobes on each side, none of which are deeply divided. The siphonal lobe is longer than wide, and has two very small, short, nearly parallel, obscurely bifid, terminal divisions, with a more oblique, somewhat similar branch on each of the sides above. The first lateral sinus is wider than the siphonal lobe, and nearly as long, with its extremity deeply divided by a slender, obscurely trifid, auxiliary lobe, into two very unequal, more or less sinuous, and obtusely digitate branches. First lateral lobe about half as wide as the siphonal, but somewhat shorter, and bearing two very small terminal divisions, similar to those of the siphonal lobe. Second lateral sinus not larger than the outer division of the first, and merely obscurely divided into two very short, simple, obtusely rounded, terminal subdivisions. Second lateral lobe very small, and obscurely trifid at the end. Whether this last is what is usually called a ventral lobe, or whether there is another still smaller one beyond it, the specimen is scarcely in a condition to show.

To this the writer adds the following observations of the type specimen: Ribs on youngest part of living chamber coarser and more widely spaced toward the aperture; ventral ribs on youngest part of last septate whorl faintly curved back forming a broad sinus on the venter; total number of ventral ribs on exposed whorls about 65 , of which 29 are on the living chamber; total number of primary ribs 24 , of which 10 are on the living chamber; length, $22.2 \mathrm{~mm}$; height, $16.1 \mathrm{~mm}$.; width, $8.4 \mathrm{~mm}$.

The writer has at hand 11 adult specimens of Scaphites larvaeformis and fragments of several times that number, all from a bed of limestone concretions in the basal part of the Carlile shale of the Black Hills. The adults range uniformly in length from 13 to $26.5 \mathrm{~mm}$., averaging $21.2 \mathrm{~mm}$. The living chambers have 6 to 9 primary ribs, averaging 7.5 , and 19 to 40 secondaries, averaging 26.9. Most are coarsely ribbed near the aperture and nearly all display backward bending of the ribs crossing the venter on the younger part of the last septate whorl. The young stages have a smooth subevolute shell for the first three or three and a half whorls. The whorl succeeding the protoconch is wide and depressed. In the next whorl the cross section is much more rounded. Beginning with the third whorl, the ventrolateral margin becomes subangular and is raised into a faint ridge which is broadly undulating, forming poorly defined blunt nodes. On the fourth whorl, at a diameter of between 4.5 and $5 \mathrm{~mm}$., broad weak ribs appear, trending straight across the venter. They are not of uniform strength or spacing for the next one-half whorl, but following that they become very high and uniform. Each pair of ribs unites in a ventrolateral node from which extends a single primary rib. At a diameter of about $6 \mathrm{~mm}$. a few intercalated secondaries appear on the venter. As the whorls enlarge the intercalated ribs increase in abundance, the primaries become stronger, but the ventrolateral nodes are reduced to radially elongate swellings. Four or five whorls comprise the septate part of an adult. Many specimens have a very small swelling on the umbilical shoulder at the base of the straight part of the shell.

Scaphites larvaeformis is characterized by its small size, quadrangular shape with markedly straight living chamber, row of small, pointed ventrolateral tubercles, and apicad bending of the ventral ribs on the younger part of the last septate whorl. In addition, many specimens show a small umbilical swelling and, near the aperture, coarser ribbing than on the older part of the living chamber.

A specimen figured as $S$. geinitzi D'Orbigny by Geinitz (1874, p. 191, pl..12, fig. 23) resembles $S$. larvaeformis in its form and in the arrangement of ribs and nodes, including the wide spacing of the ribs near the aperture, but apparently differs by possessing a large umbilical swelling. Schlüter $(1872$, p. 75 , pl. 
23, figs. $12-22$; pl. 27; fig. 5) figured a number of examples of $S$. geinitzi, all differing from $S$. larvaeformis by their larger size, more numerous nodes and primary ribs, and the tendency toward bigger umbilical swellings. A specimen figured by Schlüter (pl. 23, figs. 2325 ) as Scaphites sp. closely resembles $S$. larvaeformis in its sculpture and wide spacing of the ribs near the aperture, but differs in its more rounded outline and larger size. Yabe's S. yonekurai (1910, p. 165, pl. 15, figs. 4-7) shows a backward bending of the ribs over the venter as in S. larvaeformis, but the living chamber is much more depressed and does not have the row of nodes. . S. meriani Pictet and Campiche $(1861$, p. 16 , pl. 44) closely resembles $S$. larvaeformis in shape and general sculptural features including the apicad inclined ribs on the last septate whorl, but differs in the more depressed whorls and denser costation. The specimen figured by Collignon (1928, p. 54, pl. 5, figs. 15, 15a, 15b) as Scaphites cf: S. meriani and later referred tentatively to $S$. hugardianus D'Orbigny by Spath $(1937$, p. 503$)$ also shows rursiradiate ribbing on the sêptate whorl.

Holotype, U.S.N.M. 229; plesiotypes, U.S.N.M. 106743-106745.

The species occurs in limestone concretions in the lowest part of the Carlile shale of the Black Hills, associated with Collignoniceras woollgari (Mantell) and Inoceramus fragilis Hall and Meek.

Scaphites larvaeformis Meek and Hayden var. obesus Cobban, n. var.

Plate 1, figures 16-22

This variety differs from the typical form in its decidedly stouter shell, greater size, and tendency toward -denser costation. Because of the stoutness, the space between the curved part of the living chamber and the septate coil is proportionally smaller than in the typical form. The holotype is $31.5 \mathrm{~mm}$. long, $25.9 \mathrm{~mm}$. high, and $16.2 \mathrm{~mm}$. wide. It has 16 primary ąnd 60 ventral ribs on the exposed whorls, with 8 primaries and 29 secondaries on the living chamber. Thirteen adults from the Black Hills range in length from 21 to $37 \mathrm{~mm}$., averaging $29.8 \mathrm{~mm}$. These have on their living chambers 6 to 10 primary ribs, averaging 8 , and 24 to 45 secondaries, averaging 32.2 .

Holotype, U.S.N.M. 106767; paratype, U.S.N.M. 106768.

The holotype is from a limestone concretion in the lowest part of the Carlile shale, 5 miles southeast of Hot Springs, Fall River County, S. Dak.

$$
\begin{gathered}
\text { Scaphites patulus Cobban, n. sp. } \\
\text { Plate 1, figures 23-32 }
\end{gathered}
$$

This species is closely related to Scaphites larvaeformis from which it differs by its more rounded outline, fewer ribs, equal spacing of the ventral ribs on the living chamber, stronger tubercles, and much more depressed cross section of the living chamber. The general sculptural features, including rursiradiate ribbing on the last septate whorl, are similar to those of $S$. larvaeformis. The holotype is $31.5 \mathrm{~mm}$. long, $27.5 \mathrm{~mm}$. high, and $19.5 \mathrm{~mm}$. wide. It has 18 primary ribs and 55 secondaries on the exposed whorls, with 6 or 7 primaries and 27 secondaries on the living chamber. The 10 other adults at hand range in length from 23 to 43 $\mathrm{mm}$. and show 5 to 8 primaries and 21 to 27 secondaries on the living chambers.

The suture is similar to that of $S$. larvaeformis.

Holotype, U.S.N.M. 106769; paratypes, U.S.N.M. 106770, 106771.

The species occurs with $S$. larvaeformis, Collignoniceras woollgari (Mantell), and Inoceramus fragilis Hall and Meek, in the lowest part of the Carlile shale of the Black Hills. The holotype is from a limestone concretion found two miles southeast of Fairburn, Custer County, S. Dak.

\section{Șcaphites praecoquus Cobban, n. sp. Plate 1, figures 33-36}

The holotype of this tiny species is only $10.4 \mathrm{~mm}$. long, $8.2 \mathrm{~mm}$. high, and $4.8 \mathrm{~mm}$. wide. It is quadrangular in side view, and moderately stout. The septate coil is not ribbed, but the subangular ventrolateral margin is undulated into large, blunt nodes. The umbilicus is wider than in most scaphites from the Carlile shale. The living chamber, which comprises most of the specimen, is uniform in size and has a very small umbilical swelling at its base. The straight part is smooth except for a few faint irregularly spaced ventral ribs. A row of small ventrolateral tubercles appears on the curved part, and the ventral ribs become progressively larger toward the aperture, with those on the last half of the. whorl relatively coarse. Primary ribs are absent. The aperture is broadly ovate and slightly constricted.

The suture was not seen.

This species could be considered a variant of Scaphites larvaeformis that developed an uncoiled living chamber before other adult features were fully developed. The septate coil is comparable to the third inner whorl of $S$. larvaeformis, and the sculpture of the curved part of the living chamber suggests the larger species. However, in the younger Carlile beds, similar tiny adult scaphites occur that are related to contemporary larger species, but are distinctly different from the smallest adult forms of those species. By Niobrara time descendants of the small species are unlike associated species. It seems best to consider $S$. praiecoquus as a 
split from $S$. larvaeformis and the initial member of a new line of scaphite development.

Flolotype, U.S.N.M. 106758:

The holotype is from a limestone concretion near the base of the Carlile shale, in the $\mathrm{NE}^{1 / 4}$ sec. $15, \mathrm{~T} .57 \mathrm{~N}$., R. 62 W., Crook County, Wyo.

\section{Scaphites arcadiensis Moreman}

Plate 2, figures 1-8

1942. Scaphites arcadiensis Moreman, Jour. Paleontology, vol. 16 , no. 2 , p. 216 , pl. 34 , fig. 3 .

This species is characterized by its small size, simple suture, relatively few but coarse ribs, and living chamber with depressed cross section, with widely spaced ventral ribs on the older part, and with about five primaries raised into strong elongate nodes. The type specimen has 17 primary ribs on the exposed whorls and only 5 on the living chamber, and 48 ventral ribs on the exposed whorls and 18 on the living chamber. It is 26 $\mathrm{mm}$. long and $21 \mathrm{~mm}$. high.

Scaphites arcadiensis is distinguished from S. patulus by its fower ribs, wide spacing of the ventral ribs toward the base of the living chamber, lack of pointed tubercles, and lack of rursiradiate ribbing on the septate coil.

Plesiotypes, U.S.N.M. 106765a-c.

The holotype is from the Arcadia Park formation, 1 mile south of Arcadia Park, Texas. In the Western Interior the species has been collected from the ferruginous concretions in the lower part of the Carlile shale of the northern Black Hills associated with Scaphites carlilensis Morrow and Collignoniceras hyatti (Stanton).

\section{Scaphites carlilensis Morrow}

Plate 2, figures 9-23

1935. Scaphites carlilensis Morrow, Jour. Paleontology, vol. 9, no. 6, p. 466 , pl. 50 , figs. $4 a-d$.

Shell of average size for the genus, oval in outline, stout, and ornamented by equispaced, straight primary and secondary ribs. Morrow gives the following dimensions and rib counts on the holotype: Length, $55 \mathrm{~mm}$.; width, $27 \mathrm{~mm}$.; total number of ventral ribs on exposed whorls, 58 ; ventral ribs on living chamber, 27

In the northern Black Hills this species occurs abundantly in ferruginous concretions in the lower part of the Carlile shale above the zone of Scaphites larvaeformis. A collection consisting largely of the living chambers of 43 adults from one locality shows a range in height from 27 to $55 \mathrm{~mm}$., averaging $39 \mathrm{~mm}$. The living chambers have 8 to 16 primary ribs, averaging 10.3 , and 24 to 38 secondaries, averaging 27.7. These specimens show normal variation for a scaphite species with the larger individuals stouter and more densely costate than the smaller.
The suture is simple and has a broad, shallow first lateral saddle and fairly short bifid lobes.

Plesiotypes, U.S.N.M. 106740, 106741, 106742a-c.

The holotype is from the Blue Hill shale member of the Carlile shale three miles southeast of Tipton, in the SE $1 / 4$ sec. 4, T. 9 S., R. 10 W., Mitchell County, Kansas, associated with Collignoniceras hyatti (Stanton). The species is known also from a widespread ferruginous concretion unit in the lower part of the Carlile shale on the north flank of the Black Hills, on the Cat Creek anticline of central Montana, and on the Sweetgrass arch of north-central Montana.

\section{Scaphites morrowi Jeletzky}

1935. Scaphites pygmaeus Morrow, Jour. Paleontology, vol. 9, no. 6 , p. 465 , pl. 50 , figs. $2 \mathrm{a}-\mathrm{e}, 3$.

1942. Scaphites pygmaeus Morrow. Moreman, Jour. Paleontology, vol. 16, no. 2, p. 216, pl. 34, figs. 5, 6 .

1949. Scaphites morrowi Jeletzky, Jour. Paleontology, vol. 23, no. 3, p. 330 .

It is possible that this form is a small, slender variant of Scaphites carlilensis Morrow. Regarding a comparison of his species, Morrow (1935, p. 466) states:

Scaphites pygmaeus and $S$. carlilensis are very much alike in many details, the principal difference being in the size. The former is consistently smaller, all specimens being very nearly the size of the figured specimens. Another constant difference is in the number of ribs crossing the venter. $S$. carlilensis having from six to ten more.

A large collection of $S$. carlilensis from the Black Hills shows a fairly uniform gradation in size from specimens as small as Morrow's S. pygmaeus to those even larger than his holotype of $S$. carlilensis. The smaller specimens have fewer ribs. However, the Black Hills specimens have uniformly spaced ventral ribs whereas Morrow's $S$. pygmaeus has the ribs more widely spaced on the straight part of the living chamber than on the curved part. The importance and constancy of this feature has not been determined.

Scaphites warreni Meek and Hayden

Plate 3, figures 8-21

1860. Scaphites warreni Meek and Hayden, Acad. Nat. Sci. Philadelphia Proc., vol. 12, p. 177.

1876. Scaphites warreni Meek and Hayden. Meek, U. S. Geol. Survey Terr. Rept., vol. 9 , p. 420 , pl. 6, fig. 5 .

1876. Scaphites warreni Meek and Hayden var. wyomingensis Meek, U. S. Geol. Survey 'Terr. Rept., vol. 9, p. 421, text figs. 61-63.

1877. Scaphites warreni Meek and Hayden. White, U. S. Geog. and Geol. Surveys W. 100th Mer. Rept., vol. 4, pt. 1, p. 200 [not pl. 19, fig. 3a].

1893. Scaphites warreni Meek and Hayden. Stanton, U. S. Geol. Survey Bull. 106, p. 185, pl. 40, fig. 4 [not figures 5-7].

1898. Scaphites warreni Meek and Hayden. Logan, Kansas Univ. Geol. Survey, vol. 4, p. 475 , pl. 104, fig. 4 [not figs. 5-7]. 
1899. Scaphites warreni Meek and Hayden. Logan, Field Mus. Nat. History Pub., Geol. ser., vol. 1, no. 6, p. 210 [not pl. 22, fig. 1; pl. 23, fig. 5].

1916. Holcoscaphites warreni (Meek and Hayden). Nowak, K.-k. Geol. Reichsanstalt Verh., Jahrg. 1916, no. 3, p. 66.

1927. Scaphites warreni Meek and Hayden. Reeside, U. S. Geol. Survey Prof. Paper 150-B, p. 36.

Meek's description (1876, p. 420 ) is as follows:

Shell small, transversely subovate, moderately compressed; inner volutions nearly circular, closely involute, and composing a comparatively rather large part of the entire bulk; deflected body-portion short and (perhaps accidentally) rather more compressed proportionally than the inner turns; surface costate, and without proper nodes; costae small on the inner volutions, where they do not differ materially in size, but on the body part about every fourth or fifth one becomes more prominent than the others, and extends entirely across.from the inner side to and over the periphery, in passing upon which they bifurcate, or give off lateral branches, so that the whole, with some intercalated ones, assume there a uniform size; aperture and septa unknown.

Length, 1.45 inches; height, about 1.22 inches; convexity, about 0.57 inch.

The foregoing description is made out from the original type specimen of $S$. warreni, which is unfortunately not in an entirely satisfactory condition, either as to form or ornamentation, while it shows no traces of the septa.

* * * I should remark that the specimen from which our figure 5 , on plate 6 was drawn, seems to have had its body-part accidentally compressed late $e_{\perp}$ ally, while its mouth or lip probably does not end where it would seem to in the figure, but may pass under the rock, and curve farther back toward the involuted part. It is also a little doubtful whether the latter has been worked out correctly, the enveloping rock adhering so firmly to the shell, that in cutting it away, the inner part of the whorl is made to appear smooth instead of costate, and it is not quite clear that this part is so broad as represented. The same difficulty of having to cut away the enveloping rock has prevented the bifurcations of the costae, particularly the larger ones, from being clearly seen.

The holotype is from the southern edge of the Black Hills. Associated with it, and preserved in the same hard calcareous siltstone, is the greater part of the living chamber of a small, slender scaphite with ventral ribs more widely spaced on the straight part than on the curved part. The holotype is not sufficiently complete to demonstrate the presence of a similar type of ribspacing. Scaphites identical with the small specimen occur widespread in the Western Interior at one horizon in the Carlile shale. These grade into specimens as large or larger than the holotype. The larger individuals are stouter and more densely ribbed than the smaller, a condition normal for a scaphite species. The small, slender form is herein set aside as $S$. warreni var. ubiquitosus. The abundant material from the Black Hills now at hand permits a more detailed description of the species and its variations.

The form typical of the species is stout, about medium size, subovate in outline, with small umbilicus and living chamber considerably freed from the septate coil. The living chamber increases in size toward the bend, then tapers toward the aperture. Venter on last septate whorl well rounded and, on living chamber, broadly rounded. Living chamber slightly depressed in cross section; venter rounded in side view; umbilical wall nearly straight on older half. Aperture reniform and only very faintly constricted; dorsal lappet broad and slightly extended. Ten adults from one locality in the Black Hills range in length from 29 to $47 \mathrm{~mm}$., averaging $35: 2 \mathrm{~mm}$.

The early whorls are broad and depressed. Up to a diameter of 4 or $5 \mathrm{~mm}$. they have a subangular ventrolateral margin which, in some individuals, is raised into a faint ridge as in Scaphites larvaeformis. Later whorls are higher and more rounded.

The early whorls are smooth. At a diameter of about $3 \mathrm{~mm}$. faint ribs appear that pass straight across the venter. These increase in height as the whorls enlarge, and at a diameter of about $5 \mathrm{~mm}$., primary ribs appear. The latter are inclined slightly forward on crossing the flank and, at the ventrolateral margin, they swell slightly and then divide into two ribs that trend straight across the venter. Intercalated secondary ribs are rare at first but become more numerous as the whorls enlarge, and on the last septate whorl there are as many as 6 secondaries for each primary rib. The sculpture of the living chamber consists of strong primary ribs and numerous weaker secondary ribs, and all are more closely spaced on the curved part. The primary ribs range from straight to somewhat sigmoid, and extend from the umbilical shoulder half way to the middle of the venter where they split into two or three secondaries. Near the ventral extremity of the primaries, two or three intercalated secondaries appear and pass straight across the venter. In some. specimens, particularly those that have a greater than average depression of the living chamber, the ventral extremity of the primary ribs is raised into an incipient elongate node. Rib counts on the living chambers of 13 adults from one locality in the Black Hills show 6 to 13 primaries, averaging 8.4 , and 27 to 41 secondaries, averaging 34.2 .

The suture is simplified to a pseudoceratitic stage with broad, slightly incised saddles, and short lobes.

Meek (1876, p. 241, text figs. 61-63) gave the name, S. warreni var. wyomingensis, to a specimen from the Medicine Bow River area of southeastern Wyoming. This specimen has been lost, but Meek's figures suggest an individual of average form and sculpture for the species.

Scaphites warreni resembles $S$. arcadiensis and $S$. morrowi in the wider spacing of the ventral ribs on the straight part of the living chamber than on the curved part, but it is easily distinguished by its weaker and 
more numerous ribs and the more simplified suture. Frech's S. warreni var. silesiaca (191.5, p. 557, figs. 3a, $3 \mathrm{~b})$ is much more densely costate, shows uniform rib spacing, and has an umbilical swelling; it should be regarded as a distinct species.

Holotype, U.S.N.M. 225; plesiotypes, U.S.N.M: 1.06746-106750.

This species occurs in the lower third of the Turner sandy member of the Carlile shale of the Black Hills associated with Inoceramus dimidius White and Prionocyclus macombi Meek. It is especially abundant in the Mancos shale of the San Juan Basin of northwestern New Mexico and southwestern Colorado.

Scaphites warreni Meek and Hayden var. ubiquitosus Cobban, n. var.

Plate 3, figures 26, 27; plate 4, figures 1-15

1877. Scaphites warreni Meek and Hayden. White, U. S. Geog. and Geol. Surveys W. 100th Mer. vol. 4, pt. 1, pl. 19, fig. 3a.

The small size and slender shell characterize this variety. The holotype is $24 \mathrm{~mm}$. long, $18.7 \mathrm{~mm}$. high, and $10.5 \mathrm{~mm}$. wide. There are 21 primary ribs and 64 secondaries on the exposed whorls, with 7 primaries and 33 secondaries on the living chamber. The collection from the Mancos shale of New Mexico from which the holotype was selected consists of 12 adults ranging in length from 20 to $31 \mathrm{~mm}$., averaging $25.8 \mathrm{~mm}$., and having on their living chambers 5 to 9 primary ribs, averaging 7.4 , and 27 to 44 secondaries, averaging 35 . A collection of 26 individuals from one locality in the Black Hills shows a similar range in size and number of primaries, but the ventral ribs on the living chamber average only 32.2 .

Holotype, U.S.N.M. 106751; paratypes, U.S.N.M. 1.06752-106754.

The types are from a thin sandstone in the Mancos shale about 150 feet below the base of the Tocito sandstone lentil.

Scaphites warreni Meek and Hayden var. haydeni Cobban, n. var. Plate 3, figures 22-25

Relatively few but exceptionally strong primary ribs characterize this-variety. The holotype is of about average size and stoutness for the typical form of the species. It is $38.8 \mathrm{~mm}$. long, $33 \mathrm{~mm}$. high, and 21.7 $\mathrm{mm}$. wide. It shows 13 primary ribs and 64 secondaries on the exposed whorls, with 4 primaries and 30 secondaries on the living chamber.

The suture is simplified to a pseudoceratitic stage. Holotype, U.S.N:M. 106761.

The type was collected by F. V. Hayden from the Medicine Bow River area in the Laramie Basin, southeastern Wyoming. This is a rare form that is known elsewhere only from central Wyoming, the Black Hills, and the San Juan Basin of northwestern New Mexico.

Scaphites veterinovus Cobban, n. sp.

Plate 3, figures 1-7

Adults of this tiny species are only 10 to $15 \mathrm{~mm}$. in length. The holotype is $11.5 \mathrm{~mm}$. long, $10 \mathrm{~mm}$. high, and $6 \mathrm{~mm}$. wide. The coiled part has a fairly wide umbilicus, rounded umbilical shoulder, narrow and slightly flattened flanks, subangular ventrolateral margin raised into a ridge, and well rounded venter. The older half of the living chamber is markedly straight, and shows broadly rounded flanks grading evenly into the rounded venter. The aperture has a small dorsal lappet and incipient lateral lappets.

Whorls less than $5 \mathrm{~mm}$. in diameter are smooth. From that diameter to the base of the living chamber, the sculpture consists of straight ventral ribs. The living chamber is crossed by 30 to 45 evenly spaced ventral ribs that die out on the flanks. The holotype has about 7 faint primary ribs on the living chamber, but many specimens show none.

The suture is very simple.

In size and ornamentation this species resembles Scaphites minutus Moreman (1942, p. 216, pl. 34, figs. $9,10)$ from the Britton formation of Texas, but that species has a wider umbilicus and is more densely ribbed.

Holotype, U.S.N.M. 106763; paratype, U.S.N.M. 106764.

This species is known only from the $S$. warreni zone in the lower part of the Turner sandy member of the Carlile shale of the Black Hills. The types are from calcareous concretions 14.5 feet above the chert pebbleshark tooth conglomerate in the Turner, five miles north of Belle Fourche, in the SW $1 / 4 \mathrm{SE} / 4 \mathrm{SW} 1 / 4$ sec. $11, \mathrm{~T} .9$ N., R. 2 E., Butte County, South Dakota.

$$
\begin{gathered}
\text { Scaphites ferronensis Cobbän, n. sp. } \\
\text { Plate 4, figures 16-29 }
\end{gathered}
$$

This species is closely related to Scaphites warreni Meek and Hayden. In size and suture these species are almost identical, and the rib arrangement is similar in that the curved part of the living chamber has more closely spaced ribs that the straight part. However, there are important differences. The ventral ribs on the living chamber of $S$. ferronensis are much more dense than those of $S$. warreni, but the primaries are fewer. On internal molds the ribbing on the curved part of the living chamber is weak, and on many specimens, this part of the shell is. smooth. The smaller and more slender individuals are commonly more nearly quadrangular in outline than similar-sized specimens of S. warreni var. ubiquitosus. 
S. ferronensis is based largely on a collection (U.S.G.S. Mes. loc 12583) of 19 adults from the Ferron sandstone member of the Mancos shale near Emery, Utah. These range in leng th from 25 to $39.5 \mathrm{~mm}$., averaging $33.1 \mathrm{~mm}$., and in height from 19 to $35.5 \mathrm{~mm}$. averaging $24.6 \mathrm{~mm}$. The ventral ribs on the exposed whorls number 86 to 99 , and the primaries 13 to 25 . The living chambers have 35 to 56 ventral ribs, averaging 46 , and 4 to 7 primaries, averaging 5.1 . The holotype is $26 \mathrm{~mm}$. long, $24 \mathrm{~mm}$. high, and $13.6 \mathrm{~mm}$. wide. It has 92 ventral ribs and 18 primaries, with 47 secondaries and 5 primaries on the living chamber.

Holotypes; U.S.N.M. 106759; paratypes, U.S.N.M. 106760a-b.

The holotype is from the Ferron sandstone member near Emery, Utah. The species also occurs in the Mancos shale 275 feet below the top on the west side of the Cebolleta Mesa, Valencia County, central western New Mexico; in the top of the Frontier sandstone member of the Mancos shale of northwestern Colorado and northeastern Utah; and in the top of the Frontier formation of the Laramie Basin, southeastern Wyoming.

Scaphites whitfleldi Cobban, n. sp.

Plate 4, figures 30-40; plate 5, figures 1-4

1880. Scaphites warreni Meek and Hayden. Whitfield, U. S. Geog. and Geol. Survey Rocky Mtn. Region Rept. on Black Hills, p. 444, pl. 13, figs. 1-4.

1880. Scaphites wyomingensis Meek. Whitfield, U. S. Geog. and Geol. Survey Rocky Mtn. Region Rept. on Black Hills, p. 446, pl. 13, figs. 5-7.

1893. Scaphites warreni Meek and Hayden. Stanton, U. S. Geol. Survey Bull. 106, pl. 40, figs. 5-7.

1898. Scaphites warreni Meek and Hayden. Logan, Kansas Univ. Geol. Survey, vol. 4, pl. 104, figs. 5-7.

1899. Scaphites warreni Meek and Hayden. Logan, Field Mus. Nat. History Pub., Geol. ser., vol. 1, no. 6, pl. 23, fig. 5.

1910. Scaphites warreni Meek and Hayden. Grabau and Shimer, North American index fossils, p. 176, fig. $1427 a, b$.

1915. Scaphites warreni Meek [and Hayden]. Frech, Centralbl. Mineralogie, Jahrg. 1915, no. 21, p. 557, figs. 4a, 4 b.

1944. Scaphites warreni Meek and Hayden. Shimer and Shrock, Index fossils of North America, p. 591, pl. 244, fig. 6 .

Adult shell of average size for the genus, quadrangular in outline, and slender to moderately stout. Sculpture consists of dense, evenly spaced ribs.

The cross section of the whorl succeeding the protoconch is wide and depressed. The second whorl is much higher and more rounded, and the next two are almost circular or, in many individuals, distinctly compressed with slightly flattened flanks. The umbilicus is wide in the first whorl and narrow in the rest. Up to a diameter of $4 \mathrm{~mm}$. the ventrolateral margin is subangular and raised into a faint ridge. Between whorl diameters of 4 and $6 \mathrm{~mm}$. this ridge becomes fainter, gradually disappearing. The living chamber, which begins after the fifth whorl succeeding the protoconch, is of nearly uniform size, with only a very small and gradual expansion toward the aperture. The umbilical area of the living chamber is broad and gently sloping on the straight part but is steeply inclined on the curved part. The aperture is reniform, slightly constricted, and has a small dorsal lappet. A collection of 12 adults from the type locality on the north flank of the Black Hills shows lengths ranging from 28 to $47 \mathrm{~mm}$., averaging $37.2 \mathrm{~mm}$., although fragments of larger individuals are known (pl. 5, fig. 1). The holotype is $35 \mathrm{~mm}$. long, $.28 \mathrm{~mm}$. high, and $13.5 \mathrm{~mm}$. wide.

The inner whorls are smooth to a diameter of $4.5 \mathrm{~mm}$., where ventral ribs appear. Primary ribs appear between diameters of 7 and $8 \mathrm{~mm}$. On the first half of the last septate whorl the primaries trend straight from the umbilicus to a point a third of the distance to the middle of the venter. There they split into two ribs, with one or two intercalated secondaries. On the last half of the septate whorl and on the living chamber, the primaries are decidedly sigmoidal and strongly inclined forward. Each primary splits into 2 or 3 secondaries, and each group of secondaries is separated by 2 to 6 intercalaries. The ribs are equally strong and evenly spaced as they cross the venter. The holotype has 24 primary ribs and 115 secondary ribs on the exposed whorls; with 8 primaries and 63 secondaries on the living chamber. As many as 133 secondary ribs are visible on an adult shell. Twelve specimens from the type locality have living chambers with 6 to 9 primaries, averaging 7.6, and 37 to 75 secondaries, averaging 52 .

The suture is simple and resembles that of Scaphites warreni, but the elements tend to be more elongate.

Scaphites whitfeldi is easily distinguished by its slender, quadrangular shape and the dense, evenly spaced ribbing. Some specimens of $S$. ferronensis are as quadrangular and densely costate as $S$. whitfieldi but differ by their unequal spacing of ribs on the venter of the living chamber. The form described by Frech (1915, p. 557, figs. 3a, b) as $S$. warreni var. silesiaca has ribbing comparable to that of $S$. whitfieldi but is oval in shape and has an umbilical swelling.

The species is named for R: P. Whitfield.

Holotype, U.S.N.M. 106735; paratypes, U.S.N.M. 12258a, 106736, 106737, 106738a-b.

The types are from calcareous and ferruginous concretions in the Turner sandy member, 251 to 264 feet above the base of the Carlile shale, six miles north of Belle Fourche, in the N 12 sec. 10, T: 9 N., R. 2 E., Butte County, South Dakota, associated with Inoceramus perplexus Whitfield and Prionocyclus wyomingensis Meek. The species has been collected from many localities 
around the Black Hills in the middle third of the Turner sandy member of the Carlile shale. It is known also from the Carlile member of the Cody shale in the Crow Indian Reservation in south-central Montana, from the top of the Frontier formation of the Laramie Basin of southeastern Wyoming, and from the Mancos shale at many localities in Colorado, Utah, and New Mexico.

Scaphites pisinnus Cobban, n. sp.

Plate 5, figures 5-8

Adult shell tiny, oval in outline, with relatively large living chamber and small septate coil. The latter has a fairly wide umbilicus, narrow flanks, subangular ventrolateral margin raised into a faint ridge, and well-rounded venter. The living chamber increases uniformly in size to the aperture. The venter and dorsum are almost evenly curved in side view. The flanks and venter are well rounded. 'The aperture is incomplete, but weakly developed lateral lappets are indicated. The holotype is $8.6 \mathrm{~mm}$. long, $7 \mathrm{~mm}$. high, and $4 \mathrm{~mm}$. wide.

The septate coil is smooth. The sculpture of the living chamber consists of about 25 equispaced ventral ribs that are arched slightly forward. About 6 primary ribs are present but scarcely discernible.

'The suture is one of the simplest known for any of the adult scaphites of the Colorado group. The ventral lobe is as broad as high, with a wide siphonal saddle. The first lateral saddle is broader than the ventral lobe and asymmetrically bifid. The first lateral lobe is bifid and half as large as the ventral lobe. The second lateral saddle is bifid and as wide as the first lateral lobe. The second lateral lobe is very small and undivided. The third lateral saddle is bifid and a little larger than the second lateral saddle. The internal suture was not seen.

This species differs from Scaphites veterinovus in its oval outline, smooth septate coil, fewer ribs on the living chamber, and larger incipient lateral lappets.

Holotype, U.S.N.M. 106762.

The species occurs with $S$. whitfieldi in the middle part of the Turner sandy member of the Carlile shale of the northern Black Hills. The holotype is from a bed of ferruginous concretions 251 to 264 feet above the base of the Carlile shale, six miles north of Belle Fourche, in the N3/2 sec. 10, T. 9 N., R. 2 E., Butte County, S. Dak.

Scaphites nigricollensis Cobban, n. sp.

Plate 6, figures 1-17; plate 7, figures 1-5

Shell elliptical in outline, moderately stout, with small umbilicus, and living chamber considerably freed from the septate coil and commonly laterally inflated at its base. The internal whorls are circular to slightly wider than high in cross section; the last whorl is usually laterally compressed. The umbilicus is wide only up to a diameter of 4 or $5 \mathrm{~mm}$. The living chamber is almost uniform in size, and has slightly flattened flanks and well-rounded venter. The aperture is faintly constricted, about as high as wide, and has small dorsal lappet. Adults are slightly larger than average for the genus. Seventeen specimens from the type locality in the Black Hills range in length from 37 to $63 \mathrm{~mm}$., averaging $51.6 \mathrm{~mm}$. 'The holotype is $54 \mathrm{~mm}$. long, 43 $\mathrm{mm}$. high, and $21 \mathrm{~mm}$. wide.

The first three and a half whorls are smooth. Weak ventral ribs first appear at. a diameter of $5 \mathrm{~mm}$. Primary ribs appear at a diameter of about $10 \mathrm{~mm}$. On the last septate whorl about two secondary ribs are present to each primary, whereas on the living chamber the ratio of secondaries to primaries is about four to one. The ribs are fairly straight and, on the venter, evenly spaced. On internal molds the ribbing is weak on the older half of the living chamber, and on many individuals the venters are smooth (pl. 6, figs. 9, 11). The secondaries extend well down between the primaries. The holotype has 30 primaries and 111 secondaries on the exposed whorls, with 14 primaries and 52 secondaries on the living chamber. Rib counts on the living chambers of 18 adults from the type locality show 9 to 15 primaries, averaging 11.4 , and 38 to 58 secondaries, averaging 49.

The suture, although fairly simple, is slightly more complex than that of Scaphites whitfieldi.

The diagnostic features of $S$. nigricollensis are the swelling at the base of the living chamber, numerous primary and secondary ribs, and the tendency toward loss of ribbing on the older half of the living chamber of internal casts. This loss of ribbing is similar to that of $S$. ferronensis, but $S$. nigricollensis differs in its larger size, uniform spacing of ventral ribs, and the greater number of primary ribs. Of European species S. nigricollensis is nearest to the form described by Frech (1915, p. 557, figs. 3a, 3b) as $S$. warreni var. silesiaca from the upper Turonian.

Holotype, U.S.N.M. 106730; paratypes, U.S.N.M. 106731a-d, 106732.

The types are from calcareous concretions 59 feet below the top of the Turner sandy member (and 294 feet above the base of the Carlile shale), six miles north of Belle Fourche, in the NE/4 NE $14 \mathrm{NW} / 1 / 4$ sec. 10, $\mathrm{T}$. 9 N., R. 2 E., Butte County, S. Dak. Outside the Black Hills region, the species occurs in the Carlile member of the Cody shale in the Crow Indian Reservation of south-central Montana, and in the Colorado shale about 650 to 750 feet below the top in north-central Montana. 
Scaphites nigricollensis Cobban var. meeki Cobban, n. var. Plate 5, figures 9-26

This variety is smaller and more slender than the typical form and the living chamber is proportionally more extended. The ribs, which are fewer and stronger, are retained on all internal molds of living chambers. The flanks of the living chamber are more rounded, and no specimen has a lateral swelling at the base. The abundant specimens show intergradation with the typical form.

The holotype is $31 \mathrm{~mm}$. long, $24.5 \mathrm{~mm}$. high, and $12.3 \mathrm{~mm}$. wide. It has 25 primary ribs and 71 secondaries on the exposed whorls, with 10 primaries and 36 secondaries on the living chamber. Thirty-two adults from the type locality range in length from 26 to 50 $\mathrm{mm}$., averaging $34.4 \mathrm{~mm}$., and have on their living chambers 7 to 14 primary ribs, averaging 10 , and 29 to 50 secondaries, averaging 40 .

The suture has narrower lobes than those of the typical form.

This variety resembles Scaphites whitfieldi in size, suture, and general sculpture, but differs in its oval shape, coarser ribbing, and living chamber with fewer ventral ribs and more primary ribs.

Holotype, U.S.N.M. 106733; paratypes, U.S.N.M. 106734a-d.

The types are from calcareous concretions 59 feet below the top of the Turner sandy member (and 294 feet above the base of the Carlile shale), six miles north of Belle Fourche, in the $\mathrm{NE}_{1 / 4}^{1 / 2 E} \mathrm{NE}_{4}^{1} \mathrm{NW}^{1 / 4}$ sec. 10, T. 9 N., R. 2 E., Butte County, S. Dak.

\section{Scaphites corvensis Cobban, n. sp. \\ Plate 7, figures 6-10}

This species is characterized by its moderately large size, stout form, slightly inflated living chamber, and suture with narrow lobes. It was derived from Scaphites nigricollensis and differs from that species in its slightly larger size, more rounded flanks, retention of the ventral ribbing on internal molds of living chambers, and in its more deeply incised suture with narrower elements. In contrast to the lateral swelling at the base of the living chamber of $S$. nigricollensis, the entire living chamber of $S$. corvensis is swollen.

The holotype is $63.5 \mathrm{~mm}$. long, $53.5 \mathrm{~mm}$. high, and $27 \mathrm{~mm}$. wide. It has 28 primary ribs and 92 evenly spaced ventral ribs on the exposed whorls, with 15 primaries and 52 secondaries on the living chamber.

Holotype, U.S.N.M. 106755.

The holotype is from a calcareous concretion 180 feet above the base of the Carlile shale member of the Cody shale, 33 miles south of Hardin, on the Crow Indian Reservation, in the $\mathrm{E}_{1} / 2 \mathrm{NW}^{1 / 4} \mathrm{SW}^{1 / 4}$ sec. 36 , T. 6 S., R. 32 E., Big Horn County, Mont. The species is known also from the Sage Breaks member and the uppermost part of the Turner sandy member of the Carlile shale of the Black Hills.

Scaphites corvensis Cobban var. bighornensis Cobban, n. var. Plate 7, figures 11-17

This variety differs from the typical form in its smaller size, more slender whorls, more depressed cross section of living chamber, and stronger sculpture with fewer ribs. The internal whorls have not been seen, but the outer whorls are well rounded in cross section and have a relatively wide and deep umbilicus. The living chamber is long, almost uniform in size, and considerably extended beyond the septate coil. The flanks and venter are rounded and intergrade evenly. The aperture is almost as high as wide, with small dorsal lappet. The holotype is $44.5 \mathrm{~mm}$. long, 36.5 $\mathrm{mm}$. high, and $20 \mathrm{~mm}$. wide.

The sculpture on the coiled part of the holotype consists of 14 primary ribs that pass straight out from the umbilicus and commonly cross the venter without forking. Well out on the flank these ribs are separated by one or two secondaries. The sculpture on the living chamber of the holotype consists of 10 primary ribs and 33 ventral ribs. The primaries are arched forward. A third of the way out from the umbilicus to the middle of the venter they become higher and branch into two or three high sharp ribs that cross the venter with a slight forward swing.

This variety is distinguished from Scaphites nigricollensis var. meeki by its stronger sculpture with fewer ribs and its more incised suture with narrower lobes.

Holotype, U.S.N.M. 106756.

The holotype is from a calcareous concretion 180 feet above the base of the Carlile shale member of the Cody shale, 33 miles south of Hardin, in the $\mathrm{E}_{1 / 2}^{1 / 2} \mathrm{NW}_{1 / 4}^{1 / 4}$ SW $1 / 4$ sec. 36, T. 6 S., R. 32 E., Big Horn County, Mont. The variety is known.also from the Sage Breaks member and the uppermost part of the Turner sandy member of the Carlile shale of the Black Hills.

\section{Scaphites preventricosus Cobban, n. sp. \\ Plate 9, figures 1-16}

Adult shell, large, stout, oval in outline, and ornamented by fairly straight primary and.secondary ribs that are uniformly spaced on the living chamber. Although most of the living chamber is freed from the septate coil, it is curved so that very little space exists between the aperture and the septate coil.

The first whorl succeeding the protoconch is wide and depressed in cross section. The second whorl is much higher proportionally than the first, and the third whorl is almost circular. Later whorls are broader than high with depressed cross sections. 
About six whorls make up the coiled part. The umbilicus is wide in the early whorls and narrow in the later. The living chamber is stout, with rounded venter and broadly rounded to slightly flattened flanks. The venter of the living chamber is curved in side view, whereas the umbilical shoulder of the older part is straight. The living chamber is almost uniform in size from its base to a point a short distance beyond the place where the umbilical shoulder begins to curve, and there it becomes depressed in cross section and gradually tapers to the aperture. The aperture is slightly constricted, a.little wider than high, and with small dorsal lappet. The adult shell is larger than average for the genus. The holotype is $75 \mathrm{~mm}$. long, $68 \mathrm{~mm}$. high, and $39.5 \mathrm{~mm}$. wide. Eleven adults from Montana and Wyoming sufficiently complete for measurement range in length from 68 to $77 \mathrm{~mm}$., averaging $74 \mathrm{~mm}$.

The coiled part is smooth up to a diameter of $3 \mathrm{~mm}$. where faint ventral ribs appear. These quickly increase in strength and become coarse about a quarter of a whorl beyond the point of first appearance. At a diameter of $8 \mathrm{~mm}$. there are about 25 of these ribs per whorl. Primary ribs appear at this diameter. They are inclined backward on crossing the umbilical wall, but on reaching the umbilical shoulder, bend forward and cross the flank with a forward inclination. The ventral ribs have a lesser forward inclination. All ribs are less curved on the younger whorls, and on the last septate whorl, they pass almost straight out from the umbilicus. The early whorls have about two secondary ribs to each primary rib, wheras the last septate whorl has 3 or 4 secondaries to each primary. The sculpture on the living chambers of ten adults consists of 36 to 49 secondaries and 6 to 15 primaries. These ribs are straight or slightly flexuous, and are evenly spaced on the venter. The holotype has 90 secondaries and 23 primaries on the exposed whorls, with 45 secondaries and 10 primaries on the living chamber.

The suture is complex and has bifid lobes. The first lateral lobe is commonly asymmetric with the dorsal branches larger than the ventral.

This species is easily distinguished from older scaphites by its larger size and more complex suture. Of the younger scaphites, Scaphites preventricosus is most closely related to $S$. ventricosus Meek and Hayden (Meek and Hayden 1862, p. 22), from which it can be distinguished by its smaller size, more extended living chamber, denser costation, and uniformly spaced ventral ribs.

Holotype, U.S.N.M.. 106675; ·paratypes, U.S.N.M. 106676 a-d, 106679.

The holotype is from a bed of calcareous concretions in the Colorado shale, 514 to 525 feet below the top, in the north bank of the Marias River, 5.5 miles south of Shelby, in the NE14 sec. 20, T. 31 N., R. 2 W., Toole County, Mont. The species is known also from many other localities in central, north-central, and northwestern Montana, in the Colorado shale 400 to 580 feet below the top. In central and western Wyoming it occurs in the upper part of the Frontier formation.

Scaphites preventricosus Cobban var. sweetgrassensis Cobban n. var.

Plate 10, figures $18-25$

This variety differs from the typical form of the species in its smaller size, more slender form, and more extended living chamber. The holotype is $60 \mathrm{~mm}$. long, $52 \mathrm{~mm}$. high, and $28.5 \mathrm{~mm}$. wide. It has 109 secondaries and 28 primaries on the exposed whorls, with 50 secondaries and 11 primaries on the living chamber. Nine adults from the type locality range in length from 51 to $67 \mathrm{~mm}$., averaging $59.7 \mathrm{~mm}$., and have 34 to 58 secondaries on the living chambers.

This variety can be readily distinguished from all earlier scaphites by its larger size and more complex suture. It can be differentiated from the slender variants of later species by the more extended living chamber.

Holotype, U.S.N.M. 106677; paratype, U.S.N.M. 106678 .

It occurs with the typical form of the species in Montana and Wyoming. The holotype is from a bed of calcareous concretions 514 to 525 feet below the top of the Colorado shale, in the north bank of the Marias River, 5.5 miles south of Shelby, in the NE $1 / 4$ sec. 20, T. 31 N., R. 2 W., Toole County, Mont.

Scaphites preventricosus Cobban var. artilobus Cobban, n: var. Plate 8, figures 1-6

This variety is based on a small collection from one locality in north-central Montana. It differs from the typical form of the species by its compressed form, flexuous ribbing, and suture with narrow lobes. The living chamber is fairly short, almost uniform in size, and considerably extended beyond the septate coil. The venter is rounded and the flanks are flattened on both the living chamber and the septate coil. The type specimen is $60 \mathrm{~mm}$. long, $50 \mathrm{~mm}$. high, and $24 \mathrm{~mm}$. wide.

The sculpture consists of numerous thin, flexuous primary and secondary ribs, with more than 100 visible on the adult. The holotype has 23 primaries and 63 secondaries on the septate coil, and 16 primaries and 49 secondaries on the living chamber.

The suture is characterized by narrow lobes. The siphonal lobe is as high as wide, with long, slender branches and narrow saddles. The first lateral saddle 
is very broad and divided asymmetrically. by a long, narrow bifid lobe, and the saddles are dissected by long, narrow lobes. The first lateral lobe is bifid and a little smaller than the siphonal lobe. The second lateral saddle is bifid and as large as the first lateral lobe. The second lateral lobe is small, bifid, and with very narrow stem and branches. Remaining elements are small and show narrow lobes.

This variety differs from Scaphites preventricosus var. sweetgrassensis by its compressed form and suture with narrower lobes. It is known only from the base of the $S$. preventricosus zone whereas the var, sweetgrassensis ranges throughout the zone. The narrow-lobed suture is much like that of $S$. corvensis and $S$. c: bighornensis, but is more incised.

Holotype, U.S.N.M. 106680.

Known only from the Colorado shale 617 to 634 feet below the top, on the north bank of the Marias River, 5.5 miles south of Shelby, in the $\mathrm{NE}_{1 / 4}^{1 / 4}$ sec. $20, \mathrm{~T} .31 \mathrm{~N}$., R. 2 W., Toole County, Mont.

Scaphites mariasensis Cobban, n. sp.

Plate 8, figures 14-17

Shell large and stout with living chamber but slightly extended beyond the septate coil. Umbilicus of average size. Umbilical wall of older half of living chamber straight for half its length and then directed outward almost at right angles. The holotype is $75.0 \mathrm{~mm}$. long, $67.5 \mathrm{~mm}$. high, and $41.0 \mathrm{~mm}$. wide.

The sculpture consists of numerous high, sharp ribs, the holotype showing 28 primaries and 81 ventral ribs on the exposed whorls, with 15 primaries and 49 ventral ribs on the living chamber. The ventral ribs on the living chamber are equispaced and extend well down on the flanks between the primaries.

The suture is relatively simple in comparison with that of the associated species, Scaphites preventricosus. The short, wide first lateral lobe has a broad supporting stem.

This species closely resembles $S$. preventricocsus in size, shape, and stoutness. The sculpture of S. mariasensis, however, is much stronger and the primaries are more numerous.

Holotype, U.S.N.M. 106681.

The species is known only from the Colorado shale, 620 to 636 feet below the top, on the north bank of the Marias River, 5.5 miles south of Shelby, in the NE1/4 sec. 20, T. 31 N., R. 2 W., Toole County, Mont.

Scaphites mariasensis Cóbban var. gracillistriatus Cobban, n. var.

Plate 8 , figures $7-13$

The holotype is an internal mold that does not preserve the inner whorls. The shell is oval in outline, slender, and of average size. It is $46 \mathrm{~mm}$. long, 36 $\mathrm{mm}$. high, and $19 \mathrm{~mm}$. wide. The septate part is crushed and incomplete. The living chamber is large in proportion to the rest of the shell and considerably extended beyond the septate coil. It is nearly uniform in size and has slightly flattened flanks and rounded venter. The aperture is about as high as wide and has a very small dorsal lappet.

The septate coil is densely costate with thin; flexuous ribs. The sculpture on the living chamber consists of 54 ventral ribs and 17 primary ribs, all narrow and sharp. The primaries are markedly flexuous, and turn sharply forward on crossing the flanks. The. secondaries begin well down on the flanks.

The suture is simple, with lobes and saddles progressively decreasing in size away from the venter.

Holotype, U.S.N.M. 106682; paratype, U.S.N.M. 106683.

The holotype is from the Colorado shale 620 to 636 feet below the top, on the north bank of the Marias River, 5.5 miles south of Shelby, in the NE $1 / 4$ sec. 20, T. 31 N., R. 2 W., Toole County, Mont.

Scaphites impendicostatus Cobban, n. sp.

Plate 11, figures 1-14

This species is characterized by its stout form, conspicuous umbilical swelling, aperture with outwardturned ventral margin, and peculiar ribs that are either flat-crested or curved back.

The first whorl succeeding the protoconch is broad and depressed in cross section. The subsequent whorls are proportionately higher, but all are broader than high. The umbilicus is wide in the early whorls and narrow in the later. The venter of the living chamber is broadly rounded and grades evenly into the flattened flanks. There is a prominent umbilical swelling at the base of the living chamber. The aperture is wider than high, slightly constricted, and has a normal dorsal lappet.' The ventral margin of the aperture, however, shows an unusual feature. It is extended slightly and bent away from the rest of the shell, and, in the holotype, there is some thickening of the shell here (pl. 11, fig. 8). The adult shell is stout, quadrangular in outline, and of about average size for the genus. The holotype is $50 \mathrm{~mm}$. long, $42 \mathrm{~mm}$. high, and $27 \mathrm{~mm}$. wide. Other specimens range in length from 37 to $55 \mathrm{~mm}$.

Ventral ribs first appear at a diameter between 3 and $4 \mathrm{~mm}$., and primary ribs appear at a diameter of about $6 \mathrm{~mm}$. These ribs are curved and spaced like the early ribs of Scaphites preventricosus. The ribs on the last septate whorl and on the straight part of the living chamber assume a form unique among the scaphites of Colorado age. As shown on plate 11, figure 7, each 
rib is strongly flattened on the distal end and produced into a flange on the aboral side. In contrast the ribs on the curved part of the living chamber are sharp and curved backwards. On the holotype the primaries on the coiled part are flat as they leave the umbilicus, and at a point a quarter of the way to the middle of the venter, they are twisted so that one of the edges becomes nearly or quite vertical. The ribs abruptly flatten again and remain so on crossing the venter where they are separated by one or two flat secondaries. On the living chamber the primaries bifurcate about a quarter of the distance from the umbilicus to the middle of the venter. The ribs of the adult shell are slightly flexuous. They are of equal strength and spocing on the venter. There are 93 secondary ribs and 29 primary ribs visible on the holotype. The living chamber has 59 secondaries and 17 primaries.

The suture is simple. The siphonal lobe is longer than wide, with a high saddle. The first lateral saddle is asymmetrically bifid and broader than the siphonal lobe. The first lateral lobe is bifid and as wide as the siphonal lobe, but only half as long. The second lateral saddle is bifid and about half as large as the first lateral saddle. The second lateral lobe is small and trifid. The third lateral saddle is bifid and slightly smaller than the second lateral saddle. The third lateral lobe is very small and bifid or trifid. The internal suture was not seen.

This species is easily distinguished from other scaphites by its form and sculpture. In size, umbilical swelling, and density of ribs it somewhat resembles the European Turonian forms figured by Leonard (1897, p. 61, pl. 6, figs. 7, 8a-b) as Scaphites lamberti Grossouvre and later referred by Frech $(1915$, p. 557) to his $S$. warreni var. silesiaca. Scaphites impendicostatus differs, however, in its flat ribs, stout form, and marked quadrangular shape. Several scaphite species have umbilical swellings and outward bending of the ventral margin of the aperture, and thus resemble $S$. impendicostatus, but all are much smaller and lack the flattened ribs. Typical examples of these scaphites are S. condoni Anderson (1902, p. 111, pl. 2, figs. 58-63) from the Cretaceous of Oregon, and the following species from the Coniacian of Japan: S.? pseudoaequalis Yabe (1910, p. 163 , pl. 15, figs. 1-3), S.? yonekurai Yabe (1910, p. 165 , pl. 15, figs. 4-7), Yezoites planus Yabe (1910, p. 167, pl. 15, figs. 12-15), and Yezoites ainuanus Shimizu (Yabe, pl. 15, fig. 16).

Holotype, U.S.N.M. 106686; paratypes, U.S.N.M. 106687-106689.

The holotype is from a calcareous concretion in the Colorado shale 195 feet above the top of a calcareous shale of Greenhorn age, 1.5 miles north of Fort Shaw, in the SE1/4 sec. 35 , T. 21 N., R. 2 W., Cascade County,
Mont. The species: is moderately abundant in the Colorado shale 500 to 550 feet below the top, on the Sweetgrass arch of north-central Montana, associated with Scaphites preventricosus. It occurs also in the upper part of the Frontier formation of the Wind River and Laramie basins of Wyoming, and in the Mancos shale of central Utah.

Scaphites impendicostatus Cobban var. erucoides Cobban, n. var. Plate 11, figures 15-28

This variety departs considerably from the typical form of the species. It is smaller, more slender, lacks an umbilicál swelling and upturned ventral apertural margin, and has fewer ribs.

The adult shell is small, ranging in length from 20 to $42 \mathrm{~mm}$. The holotype is $35.5 \mathrm{~mm}$. long, $30 \mathrm{~mm}$. high, and $18.5 \mathrm{~mm}$. wide. The shell is stout, quadrangular in side view, and with umbilicus of normal size. The living chamber is long, stout, uniform in size, and with the older half straight.

The sculpture consists of numerous high sharp primary and secondary ribs. On the living chamber the ribs are exceptionally high and the crest of each rib tends to be curved slightly back. Some large individuals have flattened ribs. The holotype has 86 ventral ribs and 33 primary ribs on the exposed whorls, of which 41 secondaries and 16 primaries are on the living chamber:

The suture is that of the species.

Holotype, U.S.N.M. 106690 ; paratypes, U.S.N.M. 106691, 106692 a-b.

The holotype is from the top of the Frontier formation at Sage Creek, in sec. 18, T. 1 S., R. 1 E., Fremont County, Wyo.

Scaphites uintensis Cobban, n. sp.

Plate 10, figures 5, 6

The collection from which the type was selected consists of crushed. specimens in shale. The holotype is an impression in the shale, and an artificial cast of it is figured.

The shell is small subovate in outline, and with narrow umbilicus. The older half of the living chamber is straight. The holotype is $26 \mathrm{~mm}$. long and 20 $\mathrm{mm}$. high.

The sculpture of the coiled part consists of numerous fairly straight primary ribs each forking at the mid-flank into two secondaries. On the living chamber the primaries consists of about 10 or 11 straight ribs which begin as prominent elongate swellings on the umbilical shoulder and terminate in a row of tubercles on the ventrolateral margin. Numerous fine ribs cross the venter of the straight part, whereas coarse distantly spaced ribs occur on the venter and flank of the curved 
part. The ratio of secondaries to primaries on the straight part is about six to one.

The suture is rather simple.

This species closely resembles Scaphites delicatulus var. greenhornensis in size, shape, and ornamentation, but can be distinguished by the incipient umbilical nodes and the higher ratio of secondary to primary ribs on the living chamber.

Holotype, U.S.N.M. 106702; paratype, U.S.N.M. 106703.

The species is known only from the Mancos shale 172 to 192 feet above the top of the Frontier sandstone member, on the south flank of the Uinta Mountains, 9.5 miles northeast of Vernal, in the $W 1 \frac{1}{2}$ sec. 11, T. 3. S., R. 21 E., Uintah County, Utah.

\section{Scaphites frontierensis Cobban, n. sp. \\ Plate 10, figures 1-4}

This species is based on a small, somewhat distorted specimen. The septate coil is normal and very densely ribbed. The living chamber is straight for half its length and largely freed from the septate coil. The sculpture on the venter of the living chamber consists of fine, closely spaced ribs on the straight part and coarse, widely spaced ribs on the curved part. Along the ventrolateral margin are six round nodes, largest on the curved part. Several subequal nodes are evenly spaced along the umbilical margin of the straight part. Obscure ribs connect these with the ventrolateral tubercles, but otherwise the flanks are smooth.

The suture is not discernible.

This species is close to Scaphites uintensis but it has fewer tubercles, and they are strongest on the curved part of the living chamber, whereas, in S. uintensis, the ventrolateral nodes are weakest on the curved part.

Holotype, U.S.N.M. 106704.

The holotype is from a sandstone bed in the Frontier formation 180 feet below the top, on Bacon Creek, in the NE $1 / 4 \mathrm{NE}_{1 / 4}^{1 / 4}$ sec. 22, T. $41 \mathrm{~N}$., R. 111 W., Teton County, Wyo.

Scaphites sagensis Cobban, n. sp.

Plate 10, figures 7-10.

Shell of average size and shape for the genus with the living chamber largely freed from the septate coil. The holotype, a distorted specimen, is $33 \pm \mathrm{mm}$. long, $27 \pm \mathrm{mm}$. high, and $23 \pm \mathrm{mm}$. wide.

The sculpture of the septate coil consists of relatively few, coarse, straight primary and secondary ribs. The living chamber likewise has relatively few ribs; there are only 6 primaries and 30 secondaries on the holotype. The ventral ribs are equispaced on both the straight and curved parts. Six ventrolateral tubercles are present on the living chamber of the holotype. The primary ribs are straight and rise into higb, elongate nodes about in the middle of the flank on the older part of the living chamber.

The suture bas not been seen.

The ventrolateral tubercles and elongate flank nodes ally this species to Scaphites uintensis and S. frontierensis, but it has fewer and more uniformly spaced ventral ribs.

Holotype, U.S.N.M. 106696; paratype, U.S.N.M. 106697.

This species is known only from the upper part of the Frontier formation of the Wind River Basin, Wyo. The holotype is from a sandstone concretion in the upper part of the Frontier formation 1.3 miles north of Sage Creek, in the SE $1 / 4$ sec. 30, T. 2 N., R. 1. W., Fremont County, Wyo.

\section{Scaphites auriculatus Cobban, n. sp. \\ Plate 10, figures 11-17}

This tiny species is represented in the collections by twelve specimens ranging in length from 7.8 to 11.5 $\mathrm{mm}$. The holotype is $9.7 \mathrm{~mm}$. long and $8.8 \mathrm{~mm}$. high.

The first and second whorls are wide and depressed in cross section. The third whorl is proportionally higher than the earlier whorls, and the fourth whorl is slightly more depressed. The umbilicus is wide in the first two whorls and narrow in the next two. The living chamber, which begins after the fourth complete whorl, is long, curved, and almost entirely freed from the septate coil. In cross section the living chamber is wider than high with broadly rounded venter and more sharply rounded flanks. The aperture is much wider than high and has dorsal, ventral, and lateral lappets. The dorsal lappet is broad and extended very little but the ventral lappet is narrower and more extended. The lateral lappets are long, narrow, and pointed, directed laterally and a little forward.

The coiled part is completely smooth or the last whorl may have weak ribs crossing the venter. This weak ribbing extends to the living chamber, but on some individuals the ribbing there is much stronger. The living chamber shows 6 to 9 large blunt primary ribs, which are the only ornamentation on some specimens. The living chamber of the holotype has 8 primaries and 31 secondaries.

The suture is simple. The siphonal lobe is a little longer than wide. The first lateral saddle is broader than the siphonal lobe and asymmetrically bifid. The first lateral lobe is bifid and half as large as the siphonal lobe. The second lateral saddle is bifid and a little wider than the first lateral lobe but only half as long. The second lateral lobe is bifid.and very small. The third lateral saddle is bifid and as large as the second lateral lobe. The third lateral lobe is undivided and. 
half as large as the second lateral lobe. The internal suture was not seen.

This species is easily distinguished by its very small size and narrow, pointed lateral lappets. It resembles Scaphites pisinnus in size and shape but can be distinguished by its weaker sculpture and more extended lateral lappets. S. auritus Schlüter (1872, p. 77, pl. 23, figs. 5-9), S. puerculus var. teshioensis Yabe (1910, p. 171, pl. 15, figs. 23-27), and S. perrini Anderson (1902, p. 114, pl. 2, figs. 71-73) bear lateral lappets but of an entirely different shape from those of S. auriculatus.

Holotype, U.S.N.M. 106684; paratype, U.S.N.M. 106685.

The species occurs in the Colorado shale 500 to 550 feet below the top, on the Sweetgrass arch, northcentral Montana, associated with $S$. preventricosus and $S$. impendicostatus. The holotype was collected from a calcareous concretion 521 to 527 feet below the top of the Colorado shale, on the north bank of the Marias River, 5.5 miles south of Shelby, in sec. 20, T. 31 N., R. 2 W., Toole County, Mont.

\section{Scaphites ventricosus Meek and Hayden}

Plate 12, figures 1-10; plate 13, figures 11-13

1862. Scaphites ventricosus Meek and Hayden, Acad. Nat. Sci. Philadelphia Proc., vol. 14, p. 22.

1876. Scaphites ventricosus Meek and Hayden. Meek, U. S. Geol. Survey Terr. Rept., vol. 9, p. 425, pl. 6, figs. 7, 8.

1893. Scaphites ventricosus Meek and Hayden. Stanton, U. S. Geol. Survey Bull. 106, p. 186, pl. 44, figs. 8, 9; pl. 45, fig. 1 [not pl. 44, fig. 10].

1898. Scaphites ventricosus Meek and Hayden. Logan, Kansas Univ. Geol. Survey, vol. 4, p. 476, pl. 104, figs. 8, 9; pl. 105, fig. 1 [not pl. 104, fig. 10].

1899. Scaphites ventricosus Meek and Hayden. Stanton, U. S. Geol. Survey Mon. 32, p. 636.

1903. Scaphites ventricosus Meek and Hayden. Douglass, Carnegie Mus. Annals, vol 2, no. 1, p. 8.

1927. Scaphites ventricosus Meek and Hayden. Reeside, U. S. Geol. Survey Prof. Paper 150-A, p. 6, pl. 3, figs. 11-18; pl. 4, figs. 1-4.

1927. Scaphites ventricosus Meek and Hayden. Reeside, U. S. Geol. Survey Prof. Paper 150-B, p. 35, pl. 10, figs. 1, 2.

1944. Scahpites ventricosus Meek and Hayden. Shimer and Shrock, Index fossils of North America, p. 591, pl. 244, fig. 10.

The bolotype is large and stout, and the umbilicus is of average size for the genus. The outer septate whorl is much wider than high, and has a broadly rounded venter and sharply rounded flanks. The living chamber is nearly circular in cross section, and in side view, the umbilical wall of the older half is nearly straight whereas the entire venter is evenly curved. The younger part of the living chamber is missing but evidently it was not in contact with the septate coil. The holotype is $79 \mathrm{~mm}$. long and 47.6 wide.
The sculpture of the holotype consists of coarse, fairly straight primary and secondary ribs. The ventral ribs are more widely spaced on the middle of the living chamber than on the extremities. The complete specimen probably had 16 or 17 primaries and about 65 secondaries on the exposed whorls and possibly 9 or 10 primaries and 34 or 35 secondaries on the living chamber. On the middle of the living chamber 4 or 5 secondaries are present to each primary.

The suture of the holotype is not preserved and Meek figured the suture of a specimen that may have been associated with the type. The suture is complex and has symmetrically bifid lobes.

Scaphites ventricosus was derived from $S$. preventricosus and differs from that species in many respects. $S$. ventricosus is larger; some individuals are more than $100 \mathrm{~mm}$. in length. As the living chamber is less freed from the septate coil, the aperture lies at an angle different from that on S. preventricosus. Commonly the lateral margin of the aperture forms a right angle with the straight part of the umbilical wall of the living chamber, whereas in $S$. preventricosus, owing to the greater enrolling of the living chamber, this angle averages about 70 degrees. The ribbing of $S$ : ventricosus is less uniformly spaced and considerably coarser than that of $S$. preventricosus.

Holotype, U.S.N.M. 1903 ; plesiotypes, U.S.N.M. 106698-106700, 106757.

The holotype is from the upper part of the Colorado shale about twenty miles northeast of Fort Benton, Mont. On the Kevin-Sunburst dome in northcentral Montana, the species occurs sparingly in the Colorado shale 300 to 400 feet below the top, associated with the coiled Inoceramus species $I$. exogyroides Meek and Hayden, I. umbonatus Meek and Hayden, and I. undabundus Meek and Hayden. In northwestern Wyoming Scaphites ventricosus is present in the lower part of the Cody shale.

\section{Scaphites tetonensis Cobban, n. sp. \\ Plate 14, figures 1-10}

This species, of about average size for the genus, is characterized by its strong, coarse ribbing, high primary ribs on the living chamber, and widely spaced ventral ribs on the older part of the living chamber. The holotype is $42.5 \mathrm{~mm}$. long, $37 \mathrm{~mm}$. high, and $25 \mathrm{~mm}$. wide.

Whorls less than $6 \mathrm{~mm}$. in diameter have not been seen. The early whorls are broad and depressed in cross section and ornamented by straight, fairly coarse ventral ribs and curved primary ribs that are inclined forward. About 30 ventral ribs are on a complete whorl $12 \mathrm{~mm}$. in diameter. The last septate whorl has a depressed cross section due to the broadly rounded venter and more sharply rounded flanks. The umbil- 
icus is fairly small and has an indistinct rounded shoulder. This whorl is much more densely ribbed than the internal whorls. The living chamber is straight for half its length, wider than high, and bas rounded flanks and venter. It is crossed by 26 to 43 straight secondary ribs and 7 to 9 flexuous primary ribs. The ventral ribs are widely spaced on the straight part and closely spaced on the curved part. The primaries are strong and attain their maximum height at the ventrolateral margin.

The holotype shows 71 ventral ribs and 23 primaries on the exposed whorls, of which 37 ventral ribs and 9 primaries are on the living chamber. The aperture is wider than high and has a very short dorsal lappet. The ventral lappet is bent outward a little.

The suture is fairly simple. The most distinctive feature is the first lateral lobe, which has a saddle for each of the two main branches about as large and as high as or higher than the central saddle.

Scaphites tetonensis was derived from S. impendicostatus by becoming less unrolled and developing fewer ribs, which are widely spaced on the straight part of the living chamber. It is associated with $S$. ventricosus but is much smaller, more slender, has stronger primaries raised into incipient nodes on the living chamber, and a much simpler suture.

Holotype, U.S.N.M. 106707; paratype, U.S.N.M. 106708.

The holotype is from a sandstone bed in the Cody shale 538 feet above the base, in the $\mathrm{NE}_{1 / 4} \mathrm{NW}^{1 / 4}$ sec. 20, T. 42 N., R. 112 W., Teton County, Wyo., where it is associated with S. ventricosus and Inoceramus umbonatus Meek and Hayden. The species is known also from the lower part of the Cody shale of the west side of the Bighorn Basin of northwestern Wyoming, and from the Mancos shale of east-central Utah.

\section{Scaphites binneyi Reeside \\ Plate 14, figures $11-16$}

1927. Scaphites vermiformis Meek and Hayden var. binneyi Reeside, U. S. Geol. Survey Prof. Paper 150-A, p. 8, pl. 6, figs. 1-8.

Shell about average size for the genus, moderately stout, with living chamber partly straightened and considerably freed from the septate coil. The lateral margin of the aperture forms an angle of 90 degrees or less with the straight umbilical wall of the older part of the living chamber. The holotype is $39 \mathrm{~mm}$. long, $35 \mathrm{~mm}$. high, and $24 \mathrm{~mm}$. wide.

The inner whorls are not visible in the specimens at hand. The last septate whorl is densely ribbed. The spacing of the ribs widens greatly on the older or straightened part of the adult living chamber and then gradually becomes closely spaced again on the younger or curved part. On the living chamber the primary ribs end in ventrolateral tubercles that are strongest where the ribs are most widely spaced.

The suture is fairly simple and closely resembles that of Scaphites tetonensis. The first lateral lobe is characterized by high lateral saddles.

Holotype, Yale Peabody Mus. 6417; plesiotypes, U.S.N.M. 106705, 106706.

This is a very rare species known only from the Cody shale of central and northwestern Wyoming. The holotype is from the Cody shale, 800 feet above the base, on the Oregon Basin anticline, in sec. 6, T. 51 N., R. 100 W., Park County, Wyo.

\section{Scaphites interjectus Reeside \\ Plate 14, figures $17-21^{\circ}$}

1927. Scaphites ventricosus Meek and Hayden var. interjectus Reeside, U. S. Geol. Survey Prof. Paper 150-A, p. 7, pl. 5, figs. 1-4 [not fig. 5].

This is a stout species whose living chamber is largely in contact with the outer septate whorl. The lateral edge of the aperture forms an obtuse angle with the umbilical wall of the living chamber. Sculpture of the living chamber consists of straight ventral ribs more widely spaced on the older part, and slightly curved primary ribs ending in ventrolateral tubercles. The suture, which is moderately incised, has large, high lateral saddles on the first lateral lobe, a characteristic feature of the scaphites of the S. tetonensis-S. binneyi group.

Holotype, Yale Peabody Mus. 6416; plesiotype, U.S.N.M. 106701.

The species is rare. The holotype is from the Cody shale 800 feet above the base, in Oregon Basin, sec. 6, T. 51 N., R. 100 W., Park County, Wyo. Reeside records it also from the Garland anticline, 31 miles northeast of Oregon Basin. One specimen (pl. 14, figs. 17-21) was collected from the Cody shale of the Lander area in the Wind River Basin, Wyo.

\section{Scaphites depressus Reeside}

Plate 15, figures 6-8

1927. Scaphites ventricosus Meek and Hayden var. depressus Reeside, U. S. Geol. Survey Prof. Paper 150-A, p. 7, pl. 5, figs. 6-10.

This is one of the biggest scaphites of Colorado age. The adults range in length from 73 to $94 \mathrm{~mm}$. All specimens are exceptionally stout and have the last septate whorl very deressed. In the largest individuals the entire adult living chamber is in contact with the septate coil. The umbilicus is of average size for the genus. The adult living cbamber attains its greatest width in the older half, then tapers evenly to the aperture. The venter of the living chamber is uniformly curved; the umbilical wall is gently curved for 
half its length and is then straightened and directed outward forming an obtuse angle with the older part. The aperture is wider than high and has a very small dorsal lappet. The inner whorls are stout; the last septate whorl is so depressed that the width of the shell is as great as or greater than the height.

The early whorls are somewhat coarsely ribbed, but the last septate whorl is finely ribbed. From the beginning of the living chamber, the ribbing gradually becomes more widely spaced to a point about midway to the aperture, then gradually becomes denser toward the aperture. On the older part of the living chamber there are five or six secondaries for each primary.

The suture is complex. The first lateral lobe is bifid, with the dorsal branch commonly a little longer than the ventral branch.

This species is most closely related to Scaphites ventricosus and Clioscaphites montanensis var. hesperius, n. gen. and sp. It differs from $S$. ventricosus in its greatly depressed outer septate whorl, denser costation, more tightly coiled shell, and lateral margin of the apertiure directed outward at an obtuse angle to the umbilical wall of the older part of the living chamber. It differs from Clioscaphites montanensis var. hesperius mainly in having a larger umbilicus and in lacking uniform spacing of the ventral ribs.

Holotype, Yale Peabody Mus. 6417; plesiotype, U.S.N.M. 106693.

The holotype is from the Cody shale, 800 feet above the base, in sec. 6, T. 51 N., R. 100 W., Park County, Wyo. It is known also from other localities in the Bighorn Basin of northern Wyoming and southern Montana, where it is associated with Inoceramus umbonatus Meek and Hayden, Baculites asper Morton, B. codyensis Reeside, and Texanites shoshonensis (Meek).

\section{Scaphites depressus Reeside var. stantoni Reeside}

Plate 15, figures 1-5

1893. Scaphites ventricosus Meek and Hayden. Stanton, U. S. Geol. Survey Bull. 106, p. 186, pl. 44, fig. 10 [not pl. 44, figs. 8 , 9 ; pl. 45 , fig. 1 ].

1898. Scaphiles ventricosus Meek and Hayden. Logan, Kansas Univ. Geol. Survey, vol. 4, p. 476, pl. 104, fig. 10 [not pl: 104, figs. 8,9 ; pl. 105, fig. 1].

1927. Scaphites ventricosus Meek and Hayden var. stantoni Reeside, U. S. Geol. Survey Prof. Paper 150-A, p. 7, pl. 3, figs. 19, 20; pl. 4, figs. 5-10.

This variety differs from the typical form of the species in its smaller size, more slender shape, and in having the younger part of the living chamber slightly separated from the septate coil. The holotype is 59.2 $\mathrm{mm}$. long, $49 \mathrm{~mm}$. high, and $34.6 \mathrm{~mm}$. wide. Other specimens from Wyoming range from 48 to $70 \mathrm{~mm}$. in length.
The sculpture on the adult consists of numerous fine and fairly straight primary and secondary ribs. the ribbing is dense on the last septate whorl and on the younger part of the living chamber. The holotype has 97 ventral ribs and 31 primary ribs on the exposed whorls of which 55 ventral ribs and 16 primaries are on the living chamber. Other specimens from Wyoming have 75 to 103 ventral ribs on the adult shell with 11 to 16 primaries and 45 to 56 secondaries on the living chamber. On the middle part of the living chamber there are 4 or 5 ventral ribs for each primary.

The suture is moderately complex.

Holotype, U.S.N.M. 18817; plesiotype, U.S.N.M. 106695.

The holotype is from the upper part of the Colorado shale on Cinnabar Mountain near Gardiner, Park County, Mont. The variety is common in the lower half of the Cody shale of the Bighorn Basin, Wyo., and in the Colorado shale of southern and southwestern Montana.

\section{Scaphites depressus Reeside var. oregonensis Reeside}

1927. Scaphites ventricosus Meek and Hayden var. oregonensis Reeside, U. S. Geol. Survey Prof. Paper 150-A, p. 7, pl. 6, figs. 11-15.

This form is much like Scaphites depressus var. stantoni in size and shape but has higher, thin, sharp ribs and higher primaries.

Holotype, Yale Peabody Mus. 6411.

The variety occurs with the typical form of the species and with $S$. depressus var. stantoni in northern Wyoming and southern Montana. The holotype is from the Cody shale, 800 feet above the base, in sec. 6 , T. 51 N., R. 100 W., Park County, Wyo.

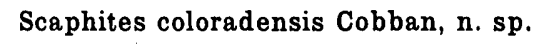

Scaphites coloradensis Cobban, n. sp.

Plate 18, figures 1-6

This species closely resembles Scaphites auriculatus in size, shape, and in the details of the aperture, but the first lateral lobe of the suture is trifid rather than bifid. The living chamber is broader and more depressed than that of $S$. auriculatus, and the sculpture tends to be more pronounced. The holotype is 9.8 $\mathrm{mm}$. long, $8 \mathrm{~mm}$. high, and $5 \mathrm{~mm}$. wide. On the living chamber are 22 coarse ventral ribs and 6 or 7 scarcely discernible primary ribs. Three other specimens from the type locality range in length from 7.7 to $12.3 \mathrm{~mm}$.

Holotype; U.S.N.M. 106715; paratype, U.S.N.M. 106714.

This species is known only from the Colorado shale 234 to 252 feet below the top, on the Kevin-Sunburst dome of north-central Montana. The holotype is from a calcareous concretion on the east bank of the Marias River, in the NE $1 / 4 \mathrm{SE} 1 / 4$ sec. $14, \mathrm{~T} .31$ N., R. 4 W., Toole County, Mont. Associated fossils 
include Clioscaphites vermiformis (Meek and Hayden) and C. montanensis, n. sp.

\section{Scaphites leei Reeside \\ Plate 21, figures 24-26.}

1927. Scaphites leei Reeside, U. S. Geol. Survey Prof. Paper 151, p. 26, pl. 20, figs. 17-22; pl. 21, figs. 1-7.

This small species is characterized by its stout form, simple suture, and living chamber with four umbilical nodes, smooth flanks, and row of ventrolateral nodes. It is distinctly different from its associates, Clioscaphites novimexicanus (Reeside), and Desmoscaphites erdmanni n. sp., of the uppermost part of the Colorado shale, and represents the first appearance of the $S$. hippocrepis-like scaphites that dominate the Telegraph Creek and Eagle faunas.

Holotype, U.S.N.M. 73354; plesiotypes, U.S.N.M. 106720 a-b.

The holotype is from the uppermost part of the Mancos shale, one mile southwest of Waldo, Santa Fe County, New Mexico. The species is known also from the uppermost part of the Mancos shale of eastcentral Utah, from the Steele shale of central Wyoming, and from the Colorado shale 10 feet below the top, on the Sweetgrass arch of north central Montana.

Genus CLIOSCAPHITES Cobban, n. gen.

Type species.-Clioscaphites montanensis Cobban.

Etymology.-From the Greek kleio, close. A closed or tightly enrolled scaphite in contrast to the typical open forms with living chambers freed from the septate coil.

The distinguishing generic characters are the closely coiled shell, the dorsum of the adult living chamber entirely in contact with the septate coil, and the suture with the trifid or asymmetrically bifid first lateral lobe.

\section{Clioscaphites montanensis Cobban, n. sp.}

Plate 16, figures $1-11$; plate 17 , figures $1-3$; plate 20 , figures $1-4$

Flattened flanks, small umbilicus, rather dense evenly spaced ribs, and complex suture characterize this species. The adults are large, attaining lengths as great as $105 \mathrm{~mm}$. Sixteen adults from the type locality average $69.2 \mathrm{~mm}$. in length; the smallest is $46.5 \mathrm{~mm}$. long. The holotype is $61 \mathrm{~mm}$. long, 52.5 $\mathrm{mm}$. high, and $32.5 \mathrm{~mm}$. wide.

The shell is tightly coiled and only a few small, slender specimens have a little of the oral end of the living chamber free from the septate whorl. In side view the venter of the living chamber is nearly evenly curved; the umbilical wall is straight for half its length and then directed outward, forming a large obtuse angle. An unusual feature is the migration of the flank upon the umbilicus. In some specimens, such as the holotype (pl. 16, fig. 1), the umbilicus is largely covered. The aperture is nearly circular and almost lacks a dorsal lappet.

The sculpture consists of numerous straight evenly spaced primary and secondary ribs, the holotype having 32 primaries and 96 secondaries on the exposed whorls with 20 primaries and 66 secondaries on the living chambers. The living chambers of thirteen adults from the type locality show 13 to 20 primaries, averaging 17.3 , and 51 to 69 secondaries, averaging 61.5. On the living chamber 4 or 5 secondaries are present for each primary. The secondaries extend well down between the primaries. There is a tendency, particularly in the larger individuals, for the primaries to attain their greatest height at the margin of the venter.

The suture is deeply incised and reaches the highest degree of complexity of the scaphites of the Colorado group. The first lateral lobe is in a transitional stage from bifid to trifid owing to elongation of the dorsal terminal branch and atrophy of the ventral branches.

Large individuals approach Scaphites interjectus in size, general form, and in having the primaries highest at the margin of the venter. However, Clioscaphites montanensis has more flattened and extended flanks; more tightly enrolled shell, denser costation, and differs in sutural details. A few smaller and more slender specimens with fewer ribs having the living chamber near the aperture slightly freed from the septate coil, and thus resemble the varieties stantoni and oregonensis of Scaphites depressus. These small specimens can be distinguished by the more flattened flanks of the living chamber, smaller umbilicus, and complex suture with a highly asymmetrical first lateral lobe.

Holotype, U.S.N.M. 106716; paratypes, U.S.N.M. 106717 a-d, 106726 a-d.

The types are from calcareous concretions in the Colorado shale, 234 to 252 feet below the top, on the east bank of the Marias River, 11 miles southwest of Shelby, in the W1/2 NE $1 / 4 \mathrm{SE} 1 / 4$ sec. 14 , T. 31 N., R. 4 W., Toole County, Mont., where they are associated with Clioscaphites vermiformis (Meek and Hayden), Scaphites coloradensis, n. sp., and Baculites codyensis Reeside. Other specimens have been collected from the upper part of the Colorado shale at several localities in northwestern, north-central, and central Montana, and a little above the middle of the Cody shale of the Bighorn and Wind River Basins of Wyoming.

Clioscaphites montanensis Cobban var. hesperius Cobban, n. var.

Plate 16, figures 12-14; plate 17, figures 4-7

This variety differs from the typical form of the species in its much more inflated form, less flattened flanks, which are little advanced over the umbilicus, 
and fewer and coarser ribs. The type is $74.5 \mathrm{~mm}$. long, $61 \mathrm{~mm}$. high, and $43.5 \mathrm{~mm}$. wide.

Some specimens of Scaphites depressus rather closely resemble this variety in shape and ribbing but are distinguishable by the larger umbilicus, the lack of equal-spaced ventral ribs, and by sutural details.

Holotype, U.S.N.M. 106718; paratype, U.S.N.M. 10671.9.

This variety is known only from the upper part of the Colorado shale of the Sweetgrass arch of northcentral Montana. The holotype is from a bed of calcareous concretions 234 to 252 feet below the top of the Colorado sbale, in the east bank of the Marias River, 11 miles southwest of Shelby, in the $\mathrm{W}^{1 / 2} \mathrm{NE} / 4 \mathrm{SE} 1 / 4$ sec. 14 , T. 31 N., R. 4 W., Toole County, Mont.

\section{Clioscaphites vermiformis (Meek and Hayden) \\ Plate 18, figures 7-27}

1862. Scaphiles vermiformis Meek and Hayden, Acad. Nat. Sci. Philadelphia, vol. 14, p. 22.

1876. Scaphites vermiformis Meek and Hayden. Meek, U. S. Geol. Survey Terr. Rept., vol. 9, p. 423, pl. 6, fig. 4.

1893. Scaphites vermiformis Meek and Hayden. Stanton, U. S. Geol. Survey Bull. 106, p. 183, pl. 44, fig. 3.

1.898. Scaphites vermiformis Meek and Hayden. Logan, Kansas Univ. Geol. Survey, vol. 4, p. 474, pl. 104, fig. 3.

1910. Scaphites vermiformis Meek and Hayden. Grabau and Shimer, North American index fossils, p. 176, fig. 1427c.

1916. Holcoscaphites vermiformis (Meek and Hayden). Nowak, IK.-k. Geol. Reichsanstalt Verh., Jahrg. 1916, no. 3, p. 66. 1927. Scaphites vermiformis Meek and Hayden. Reeside, U. S. Geol. Survey Prof. Paper 150-A, p. 7, pl. 6, figs. 9, 10.

Meek fully described the adult shell which came from the upper part of the Colorado shale of north-central Montana. He apparently saw only the single specimen for he does not mention the internal whorls, the growth stages, or variations of the adult. The writer has at hand more than a hundred specimens from north-central Montana, which makes possible a more detailed description of the species and variations.

All whorl sections are broader than high and very depressed. The venter is well rounded on the early whorls, broadly rounded to flattened on the last septate whorl, and broadly rounded on the living chamber. The flanks are rounded on the early whorls and flattened on the later whorls and body chamber. The ventrolateral margin is rounded on the first two whorls out to a diameter of about $1.5 \mathrm{~mm}$. It is subangular between whorl diameters of 1.5 to $6 \mathrm{~mm}$. and then becomes rounded on the remainder of the shell. The umbilicus is wide in the early whorls and narrow in the later ones; umbilical shoulder rounded. The living chamber is curved and not freed from the septate coil; its flanks are nearly flat and inclined in ward. The aperture is constricted, subcircular, and has a very small dorsal lappet. The adult shell is subcircular in side view, slender to moderately stout, and a little larger than average size for the genus. The holotype is $53.8 \mathrm{~mm}$. long, $.45 .1 \mathrm{~mm}$. high, and $32 \mathrm{~mm}$. wide (from extremities of nodes). Nineteen.adults from one locality in north-central Montana range in length from 25 to $57 \mathrm{~mm}$., averaging $50.7 \mathrm{~mm}$.

The early whorls are smooth to a diameter of about $4 \mathrm{~mm}$., where coarse, rounded ventral ribs appear. These are sigmoid, curving back from the ventrolateral margin and then forward on crossing the venter. There are 23 to 29 ribs on the first completely ribbed whorl. Primary ribs first appear between diameters of 5 and $6 \mathrm{~mm}$. and curve forward on crossing the narrow flank. The coarse ventral ribbing continues to the beginning of the last septate whorl where the ribs suddenly become sharp, dense, and straight. This dense ribbing continues nearly to the younger end of this whorl where the ribs gradually become more widely separated. The ribbing is sparse on the older half of the living chamber, but on the younger half it again becomes dense. The primary ribs on the living chamber are high, sharp, and nearly straight, and each terminates at the margin of the venter in a high sharp tubercle. These tubercles are largest in the middle third of the living chamber and become progressively smaller toward the extremities, disappearing near the aperture and on the youngest part of the last septate whorl. On the holotype the nodes are arranged on each side of the venter in alternate positions, but in most individuals the nodes are paired. The holotype has 23 primary ribs and 74 secondary ribs on the exposed whorls, with 14 primaries and 45 secondaries on the living chamber. The living chambers of 19 specimens from one locality in northern Montana have 10 to 16 primaries, averaging 13 , and 33 to 53 secondaries, averaging 40.7. On the middle part of the living chamber there are 3 ventral ribs to each primary.

The suture is fairly complex and characterized by trifid lobes. The siphonal lobe is almost as wide as high. The first lateral saddle is asymmetrically bifid and wider than the siphonal lobe. The first lateral lobe is trifid and as broad but not as high as the siphonal lobe. The second lateral saddle is bifid and about as wide as the first lateral saddle but only half as long. The second lateral lobe is trifid and about half as large as the first lateral lobe. The remaining lobes are small and trifid. The rest of the saddles are small and bifid.

The diagnostic features of this species are the closely coiled shell, completely curved living chamber, row of ventrolateral tubercles, and fairly complex suture with trifid lobes. Immature specimens can be distinguished from juveniles of the associated species, Clioscaphites 
montanensis, by the forward bending of the ribs as they cross the venter. In addition, most young specimens of C. vermiformis are stouter.

A few individuals (pl. 18, figs. 20, 21) have sharp, knife-like ribs and nodes and, in this respect, exactly parallel the development of Scaphites depressus var. oregonensis Reeside.

Holotype, U.S.N.M. 1902; plesiotypes U.S.N.M. $106713 \mathrm{a}-\mathrm{f}$.

The species is present in the Mancos shale of northwestern New Mexico, western Colorado, and central and northeastern Utah; in the Hilliard shale of southwestern Wyoming; in the Cody shale of central and western Wyoming; and in the Colorado shale of Montana. The holotype is from the upper part of the Colorado shale at Chippewa Point on the Missouri River about 20 miles below Fort Benton in Chouteau County, Mont.

Clioscaphites vermiformis (Meek and Hayden) var. toolensis Cobban, n. var.

Plate 19, figures 1-10

This variety differs from the typical form of the species in its larger size, stouter form, and greatly depressed whorls. In addition, the sculpture is not as pronounced and the ribbing is slightly more dense. The holotype has 26 primaries and 73 secondaries on the exposed whorls, with 14 primaries and 41 secondaries on the living chamber. Seven other specimens from the type locality have 13 to 19 primaries on the living chambers, averaging 15.6 , and 38 to 57 secondaries, averaging 45. The holotype is $68.5 \mathrm{~mm}$. long, $62 \mathrm{~mm}$. high, and $44.5 \mathrm{~mm}$. wide. Other specimens from the type locality range from 63 to $78 \mathrm{~mm}$. in length. Probably, a few adults (pl. 19, fig. 9) attained the size of Scaphites depressus.

Many individuals (pl. 19, figs. 7, 8) from the Cody shale of southern Montana and northern Wyoming differ from the northern Montana specimens in possessing denser ribbing and more depressed whorls. The last septate whorl of some individuals is so depressed as to form a broad, shallow depression along part of the venter exactly as in the most depressed specimens of Scaphites depressus.

The variety resembles the form described by McLearn (1929, p. 77, pl. 18, figs. 1-3; pl. 19, figs. 1, 2) as Scaphites ventricosus Meek and Hayden var. saxitonianus in size, shape, stoutness, and arrangement of ribbing, but differs in possessing prominent tubercles and a suture with well-developed trifid lobes.

Holotype, U.S.N.M. 106709; paratypes, U.S.N.M. 106710-106712.

The holotype is from a bed of calcareous concretions 234 to 252 feet below the top of the Colorado shale, in the east bank of the Marias River, 11 miles southwest of Shelby, in the W $1 / 2 \mathrm{NE}_{4} \mathrm{SE} / \frac{1}{4}$ sec. $14, \mathrm{~T} .31 \mathrm{~N} ., \mathrm{R}$. 4 W., Toole County, Mont. The variety occurs with the typical form of the species at many other localities in Montana and Wyoming.

Clioscaphites platygastrus Cobban, n. sp.

Plate 20, figures 12-16

The holotype is an adult shell $52.5 \mathrm{~mm}$. long, $50 \mathrm{~mm}$. bigh, and $28.6 \mathrm{~mm}$. wide. It is tightly coiled, with very small umbilicus. The living chamber, of nearly uniform size, is entirely in contact with the septate coil. The venter is flattened and the flanks but slightly convex.

The sculpture consists of numerous strong, sharp ribs and a row of high, pointed ventrolateral tubercles. The holotype has 90 ventral ribs and 28 primaries on the exposed whorls, with 70 ventral ribs and 20 primaries on the living chamber. On the septate coil both primary and secondary ribs are straight. On the living chamber the primaries bend slightly forward on leaving the umbilical wall, then curve back a little and cross the flanks gradually bending forward again. Each primary rib terminates in a ventrolateral tubercle that is curved slightly back. The ventral ribs are numerous, strong, and straight. They are more sparse on the older part of the living chamber where the ventrolateral tubercles are largest.

The suture is moderately complex with the first lateral lobe transitional from bifid to trifid.

The species closely resembles Clioscaphites vermiformis in size, shape, and general sculptural features, but differs in the flattened venter and denser and stronger costation.

Holotype, U. S. N. M. 106729.

The holotype is from the upper part of the Colorado shale, four miles west of Sunburst, in sec. 16, T. $36 \mathrm{~N}$., R. 3 W., Toole County, Mont.

\section{Clioscaphites saxitonianus (McLearn)}

Plate 13, figures 1-10

1929. Scaphites ventricosus Meek and Hayden var. saxilonianus McLearn, Canada Nat. Mus. Bull. 58, p. 77, pl. 18, figs. 1-3; pl. 19, figs. 1, 2.

Shell, large, stout, and tightly coiled. McLearn gives $59 \mathrm{~mm}$. as the length of the holotype.

The sculpture of the septate coil consists of fine, dense, straight ribs. On the living chamber the ventral ribs are coarse and sparse on the older portion and progressively denser toward the aperture. The primary ribs on the living chamber are strong and attain their greatest height in incipient nodes at the ventrolateral margin.

The suture is only moderately complex. The first lateral lobe is typically asymmetrically bifid but on advanced individuals it may be trifid. 
This species resembles Clioscaphites vermiformis va. toolensis in size, form, and general sculptural fentures, but differs in lacking pointed nodes and in rarely having the first lateral lobe of the suture trifid.

Holotype, Nat. Mus. Canada 9041; paratype, N. M. C. $9041 a$; plesiotypes, U.S.N.M. 106739a-b.

The holotype is from the Alberta shale of the Crowsnest River area of southwestern Alberta. In the United States the species is known only from the Apishapa shale of southeastern Colorado, and from the Colorado shale, 165 feet below the top, on the east flank of the Sweetgrass arch of north-central Montana.

Clioscaphites saxitonianus (McLearn) var. keytei Cobban, n. var.

Plate 20, figures 5-7

This variety differs from the typical form by its much smaller size and more slender shape. The holotype is $42.3 \mathrm{~mm}$. long, $38 \mathrm{~mm}$. high, and $27 \mathrm{~mm}$. wide. It has 20 primary ribs and 66 ventral ribs on the exposed whorls with 9 primaries and 29 ventral ribs on the living chamber.

The suture is not discernible on the type specimen. Most specimens in the type lot have the first lateral lobe asymmetrically bifid, owing to elongation of the dorsal branch.

The variety closely resembles small adults of Clioscaphites vermiformis, but lacks pointed nodes and the perfectly trifid first lateral lobe.

The variety is named for I. A. Keyte, late professor of geology at Colorado College, who. collected the type specimen.

Holotype, U.S.N.M. 106727.

The holotype is from a calcareous concretion in the Apishapa shale, 16 miles east of Trinidad, in sec. 1, T. 32 S., R. 62 W., Las Animas County, Colo. The variety occurs also in the Raton Park area of northeastern New Mexico, and in the Colorado shale, 165 feet below the top, on the east flank of the Sweetgrass arch of north-central Montana.

\section{Clioscaphites novimexicanus (Reeside)}

Plate 21, figures 1-9

1927. Desmoscaphiles novimexicanus Reeside, U. S. Geol. Survey Prof. Paper 151, p. 17, pl. 11, figs. 1-4.

This species was based on a single specimen consisting chiefly of the septate coil. The sculpture of this specimen consists of forwardly inclined primary ribs which pass half way outward from the umbilicus to the middle of the venter and then split into two coarse secondary ribs that pass straight across the venter. Constrictions were doubtfully reported on this specimen, but Dr. Reeside and the writer on reexamining the type agree that their presence cannot be demonstrated. The upper 23 feet of the Colorado shale on the west flank of the Sweetgrass arch of north-central Montana has yielded scaphites that exactly fit Reeside's figures of this species. The Montana material includes some adults, permitting more complete description of the species.

Clioscaphites novimexicanus is characterized by its compressed, involute form with very narrow umbilicus, dense costation, and suture with trifid first lateral lobe. The adults at hand range in length from $38 \mathrm{~mm}$. to $76 \mathrm{~mm}$.

The first whorl is broad and depressed in cross section; succeeding whorls are proportionally higher than the first, but all are wider than high. The umbilicus is wide in the first two whorls and narrow in the later ones. The ventrolateral margin is subangular, beginning with the third whorl at a diameter of about $2 \mathrm{~mm}$. and continuing into or through the fourth whorl to a diameter of 6 to $8 \mathrm{~mm}$. It is rounded at larger diameters. The living chamber begins with or after the sixth whorl, is relatively compressed, and is wholly attached to the septate coil. The venter is evenly curved in its entire length, is rounded in cross section, and grades uniformly into the flattened flanks. The umbilicus of the adult is exceedingly narrow and straight because of the considerable overlap by the extended flanks. The aperture is constricted, almost circular, and has scarcely any dorsal lappet.

The earliest three whorls are smooth. At a diameter of $3.5 \mathrm{~mm}$. straight, coarse ventral ribs appear, and there are about 22 ribs on the first complete whorl following their initial appearance. At a diameter of 5 $\mathrm{mm}$. primary ribs appear. These are curved and inclined forward as they cross the flanks, and on reaching the margin of the venter, they become more elevated and commonly fork into two secondaries. On the last half of the outer septate whorl the sculpture changes rather suddenly from coarse rounded ribs to sharp narrow ribs that are more closely spaced. The ribbing is especially dense on the living chamber, which has 60 to 70 equispaced straight ventral ribs and 16 to 20 straight primary ribs. Six or seven secondaries are present to each primary on the middle part of the living chamber. The secondaries are exceptionally long, extending between the primaries almost to the umbilical wall.

The suture is moderately complex and characterized by a trifid first lateral lobe. The suture is considerably simplified from its Clioscaphites montanensis ancestor.

Clioscaphites novimexicanus can be distinguished from advanced specimens of $C$. montanensis by its more compressed form, denser costation, and simplified suture with a trifid first lateral lobe. A few fragments of a large, stout variety (pl. 21, fig. 9) are present in the 
collections, but none are sufficiently complete to warrant description.

Holotype, U.S.N.M. 73312; plesiotypes, U.S.N.M. 106721a-b, 106722a-b, 106723.

The holotype is from the uppermost Mancos shale, one mile east of head of Canyon del Yeso, Santa Fe County, north-central New Mexico. The species is common in the upper 23 feet of the Colorado shale on the Sweetgrass arch, north-central Montana, associated with Inoceramus lundbreckensis McLearn and Baculites thomi Reeside.

Clioscaphites? choteauensis Cobban, n. sp.

Plate 20, figures 8-11

Shell moderately large, stout, and tightly coiled, with small umbilicus. Last septate whorl considerably depressed, with broadly rounded venter and more sharply rounded flanks. In side view both the venter and umbilical shoulder of the living chamber are evenly curved; in cross section the venter is well rounded, the flanks are flattened and round gradually into the convex, steeply sloping umbilical wall. The living chamber tapers slightly toward the nearly circular aperture. The holotype is $64.5 \mathrm{~mm}$. long, $64 \mathrm{~mm}$. high, and 35 mm. wide.

The innermost whorls were not seen. The early part of the last septate whorl is coarsely ribbed but changes abruptly to fine, dense ribbing. The ribbing widens on the proximal half of the living cnamber and then gradually becomes denser toward the aperture. On most of the shell the primaries are fairly straight but near the aperture they become broadly curved and inclined forward. On the older three-fourths of the living chamber each primary ends as a conical ventrolateral tubercle. The ventral ribs on the living chamber are straight on the older part, but arch gently forward on the younger part. They extend well down between the primaries. Four or five are present for each primary on the middle part of the living chamber. On the exposed whorls, the holotype has 27 primaries and 104 secondaries with 17 primaries and 67 secondaries on the living chamber.

The suture is complex and characterized by trifid lobes.

The well-rounded venter, denser costation, and intercalation of secondary ribs between the primaries readily distinguishes this species from Clioscaphites vermiformis and $C$. platygastrus. It is a transition species between $C$. vermïformis and Desmoscaphites erdmanni Cobban, n. sp.

Holotype, U.S.N.M. 106728.

The holotype is from near the top of the Colorado shale half a mile northeast of Choteau, in sec. 19, T. 24 N., R. 4 W., Teton County, Mont. The species occurs also in calcareous concretions 162 feet below the top of the Colorado shale on the west flank of the Kevin-Sunburst dome in north-central Montana. Outside of those areas it is known only from the upper part of the Colorado shale of southwestern Montana, from the Mancos shale of northwestern Colorado, and from the Apishapa shale of eastern Colorado.

\section{Genus DESMOSCAPHITES Reeside}

Reeside (1927c, p. 16) proposed this genus with Desmoscaphites bassleri as the genotype to include moderate-sized shells with abnormal living chambers, early whorls having constrictions and coarse, forward arching ventral ribs, later whorls finer ribbed, and suture with a trifid first lateral lobe. The genus was derived from Clioscaphites vermitormis through Clioscaphites? choteauensis by acquiring constrictions in the internal whorls.

Desmoscaphites erdmanni Cobban, n. sp.

Plate 21, figures 10-23

The external whorls of this species closely resemble those of Clioscaphites? choteauensis in size, shape, and general sculptural features. Desmoscaphites erdmanni has smaller nodes and more uniformly spaced ribs, and specimens comparable to $C . ?$ choteauensis in size are commonly more.densely ribbed.

The first two whorls succeeding the protoconch are wide and depressed in cross section, but later whorls are proportionally higher and more nearly circular. The umbilicus is wide in the early whorls and narrow in the later, with rounded umbilical shoulder. The flanks and venter are well rounded in the early whorls, but on the last septate whorl the flanks become slightly flattened and the venter broadly rounded. The living chamber is shaped like that of $C$.? choteauensis. The holotype is a nearly complete but partly crushed living chamber that was probably $48 \mathrm{~mm}$. in length.

The early whorls are smooth. Between diameters of 4 and $5.5 \mathrm{~mm}$. constrictions appear on the venter and on the outer part of the flanks. These occur in pairs spaced first about four and a half per whorl but gradually increase to six per whorl. In the early whorls the orad constriction is weaker than the apicad constriction, but in the later whorls they are equal. The constrictions of a pair are closely spaced, but separated by a high rib. This rib and the constrictions bend forward on crossing the venter. At a whorl diameter of 8 to $10 \mathrm{~mm}$. ribs first appear between the pairs of constrictions and cross the venter with the same forward swing. These ribs rapidly become coarse, attaining their greatest height on the venter. On the outer part of the flank each pair commonly unites into a primary rib that curves back across the 
flank, describing an arc concave forward. These primaries disappear at the edge of the umbilicus. At the beginning of the last septate whorl the coarse ribbing suddenly becomes fine and dense, as it does in $C$. vermiformis and $C$. ? choteauensis. On the middle third of the living chamber the ribbing is a little sparser. There the primaries are long and straight and terminate in conical tubercles on the margin of the venter. From each tubercle two or three secondaries and one or two intercalated ribs cross the venter with a forward arching. The holotype has four secondaries to each primary. Fragments of larger individuals have four to six secondaries for each primary.

The suture is moderately complex with - trifid lobes.

Demoscaphites erdmanni differs from the later $D$. bassleri Reeside in possessing fewer ribs and in having those on the middle third of the living chamber sparser than on the extremities. D. bassleri has uniformly spaced ribs with 6 to 9 secondaries to each primary.

The species is named for Mr. Charles E. Erdmann.

Holotype, U.S.N.M. 106724; paratypes, U.S.N.M. $106725 \mathrm{a}-\mathrm{d}$.

$D$. erdmanni is known only from the uppermost part of the Colorado shale in north central Montana and from the upper part of the Cody shale of the Wind River Basin of western Wyoming. The holotype is from a calcareous concretion 10 feet below the top of the Colorado shale, 8 miles west of Shelby, in the NE 14 sec. 31 ., T. 32 N., R. 3 W., Toole County, Mont., associated with Clioscaphites novimexicanus, Baculites thomi Reeside, and Inoceramus lundbreckensis McLearn.

\section{REFERENCES}

Adkins, W. S., and Winton, W. M., 1919, Paleontological correlations of the Fredericksburg and Washita formations in north Texas: Texas Univ. Bull. 1945, pp. 1-128, pls. $1-21$, (issued 1920).

Andenson, T. M., 1902, Cretaceous deposits of the Pacific Coast: California Acad. Sci. Proc., 3d ser., Geology, vol. 2, pp. 1-154, pls. 1-12.

Böse, Emil, 1927, Cretaceous ammonites from Texas and northern Mexico: Texas Univ. Bull. 2748, pp. 143-350, pls. 1-19, (issued 1928).

Collignon, Maurice, 1928, 1929, Paléontologie de Madagasgar; 15, Les Céphalopodes du Cénomanien Pyriteux de Diégo-Suarez: Annales de paléontologie, tome 17, pp. 1-25 (137-161), pls. 1-5 (15-19), 1928; tome 18, pp. 25-80 $(1-56)$, pls. $6,7(1,2), 1929$.
Frech, Fritz, Ueber Scaphites, 1915, I: Centralbl. Mineralogie, Jahrg. 1915, no. 21, pp. 553-568, 14 figs.

Geinitz, H. B., 1874, Das Elbthalgebirge in Sachsen; Theil 2, Der mittlere und obere Quader, 5, Gasteropoden und Cephalopoden: Palaeontographica, Band 20, pp. 120-199, pls. $31-36$.

Leonard, R., 1897,.Die Fauna der Kreideformation in Oberschlesien: Palaeontographica, Band 44, pp. 11-70, illus.

McLlearn, F. L., 1929, Cretaceous invertebrates; Mesozoic paleontology of Blairmore region; Alberta: Canada Nat. Mus. Bull. 58, pp. 73-79, pls. 13-19.

Meeк, F. B., 1876, Invertebrate Cretaceous and Tertiary fossils of the upper Missouri country: U. S. Geol. Survey Terr. Rept., vol. 9, pp. 1-629, illus.

MeEK, F. B., and HAYDEn, F. V., 1862, Descriptions of new Cretaceous fossils from Nebraska Territory, collected by the expedition sent out by the Government under the command of Lieut. John Mullan, U. S. Topographical Engineers, for the location and construction of a wagon road from the sources of the Missouri to the Pacific Ocean: Acad. Nat. Sci. Philadelphia Proc. 1862, pp. 21-28.

Moreman, W. L., 1942, Paleontology of the Eagle Ford group of north and central Texas: Jour. Paleontology, vol. 16, no. 2, pp. 192-220, pls. 31-34, 2 text figs.

Morrow, A. L., 1935, Cephalopods from the Upper Cretaceous of Kansas: Jour. Paleontology, vol. 9, no. 6, pp. 463-473, pls. $49-53$, text figs. $1-8$.

Pictet, F. J., and Campiche, G., 1861-1864, Description des fossiles du terrain crétacé des environs de Sainte Croix, pt. 2: Materiaux pour la paléontologie suisse, ser. 3, Mon. 2, pp. 1-752, pls. 44-98.

Reeside, J. B., Jr., 1927a, Cephalopods from the lower part of the Cody shale of Oregon Basin, Wyoming: U. S. Geol. Survey Prof. Paper 150-A, pp. 1-10, pls. 1-8.

— $1927 \mathrm{~b}$; The scaphites, an Upper Cretaceous ammonitc group: U. S. Geol. Survey Prof. Paper 150-B, pp. 21-40, pls. 9-11.

- - 1927c, The cephalopods of the Eagle sandstone and related formations in the western interior of the United States: U. S. Geol. Survey Prof. Paper 151, pp. 1-87, pls. $1-45$.

Schlüter, Clemens, 1872, Cephalopoden der oberen deutschen Kreide: Palaeontographica, Band 21, pp. 1-120, pls. 1-35.

Spath, L. F., 1923-1943, A monograph of the Ammonoidea of the Gault: Pailaeont. Soc. Mon., pp. 1-783, illus.

W ARren, P. S., 1930, New species of fossils from Smoky River and Dunvegan formations, Alberta: Alberta Research Council Geol. Survey Rept. 21, pp. 57-68, pls. 3-7.

,- 1947 , Cretaceous fossil horizons in the Mackenzie River valley: Jour. Paleontology, vol. 21, no. 2, pp. 118-123, pls. $29,30$.

YABE, H., 1910, Die Scaphiten aus der Oberkreide von Hokkaido: Beitr. Paläontologie Oesterr.-Ungarns u. des Orients, vol. 23, pp. 159-174, pl. 15. 



\section{INDEX}

[Italic numbers indicate descriptions.]

Abstract.

Acknowlodgments

aequalis, Scaphiles

ainutumus, Yesoiles

aquilaensis, Scaphices

arcadiensis, Scaphites.

artilobus, scaphites preventricosi auriculatus, Scaphites. $2,3,5,7,9,30,33$, pl. 10 auritus, Scaphites.

Buculites asper codyensis . . thomi. Uussleri, Desmoscaphites............................................ 9, 10,39 Dighornensis, Scaphites corvensis.................................... 26,82, pl. 7

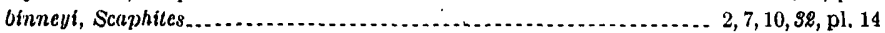
oosgueinsis, Scaphites. $2,7,10,92, \mathrm{pl}, 14$

orittonensis, Scaphiles.

6,18

Carllle shalo

carlilensis, Scaphiles.

4,5 choteauensis, Cliosctiphiles ................................ 2,4,5,6,7,10.38,39, pl. 20 circularis, Scuphites. Clioscaphites. choteauensis . ................................................ $2,4,5,6,7,10,38,39$, pl. 20 montanensig $2,3,5,6,7,8,9,10,11,34,35,36,37$, pls. $16,17,20$

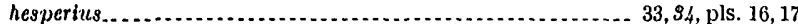
novimerticanus.............................. 2, 3,5,6,7,8,9,10,11,34, 97,39 , pl. 21 platygastrus . ................................................ $2,4,7,10,36,38$, pl. 20 saxitonianus............................................. 2,5, 7,9, $\$ 6$, pl. 13

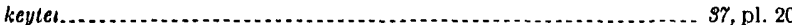
vermiformis....................................... 2, 3,5, 7, 10,34, $35,36,37,38,39$, pl. 18 toolensis........................................... 19

codyensis, Bacullites...................................................... 33, 34

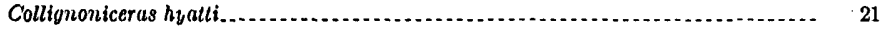

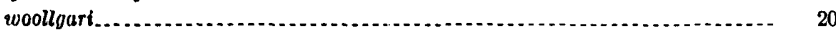
coloradensis, Scaphiles...................................... 2, 3,5,7,9, \$8,34, pl. 18

Colorado shalo. condoni, Scaphites...................................................................... 29 corvensis, Scaphites .............................. 7 bighornensis, Scaphites. $26,28, \mathrm{pl} .7$

D

delicatulus, Scaphites.

$2,3,5,6,7,9,18$

delicatulus greenhornensis, Scaphiles. depressiss, Scaphites

Scaphites ventricosus.

depresous oreyoiensis, Scaphttes. depressus stantoni, Scaphiles........................................ 9, $\$ 8,34$, pl. 15 Desmoscaphites....................................................... 2, 88

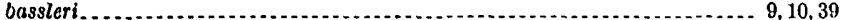
erdmanni................. $2,4,5,7,10,34,98$, pl. 21 novimexicanus...

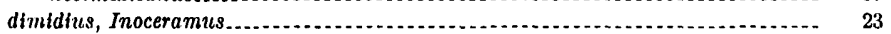

erdmanni, Desmoscaphites.................................... 2,4,5,7,10,34,38, pl. 21 erucoides, Scaphites impendicostatus................................... 11 Evolution of the scaphites............................................. 6-11 exogyroides, Inoceramus.

Serronensis, Scaphites.

$2,5,7,9,29,24,25$, pl. 4 For in and general characters

frayilis, Inoceramus...

,frontierensis, Scaphites

oeinitzi, Scaphites $18,19,20$ gracile, Sciponoceras

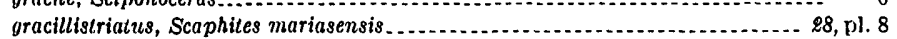
Oreenhorn formation. greenhornensis, Scaphites delicatulus. haydeni, Scaphites warreni.

H

Page hesperius, Clioscaphives montanensis. 33, $\$ 4$, pls. 16,17

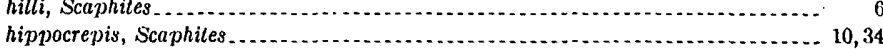

Holcoscaphites.

larvaeformis.

vermiformis.

warreni.

hugardianus, Scaphites.

hyatti, Collignoniceras

impendicostatus, Scaphites.

1

impendicostatus erucoides, Scaphites.

noceramus dimidius

exogyroides

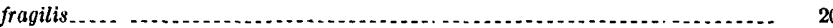

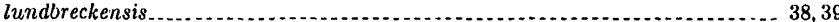

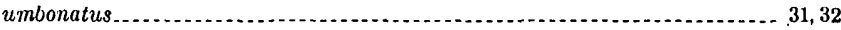

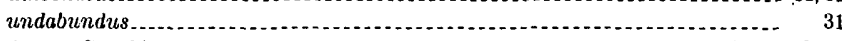

interjectus, Scaphites............................................... 10. 32,34, pl. 14

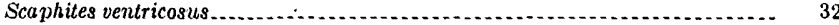

$\mathbf{K}$

keytei, Clioscaphites saxitonianus... 37, pl. 20

$L$

lamberti, Scaphites..........

larvaeformis, Holcoscaphites

Scaphites.

29

leei, Scaphites................... 21

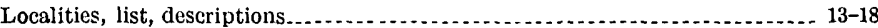

map showing. 12

$l_{\text {undbreckensis, Inoceramus } \ldots \ldots \ldots}$

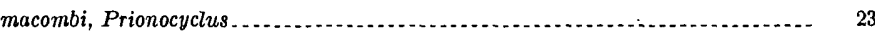

mariasensis, Scaphites.......................... 8

mariasensis gracillistriatus, Scaphites ....................................... 28, pl. 8

meeki, Scaphites nigricollensis........................................ 26, pl. 5

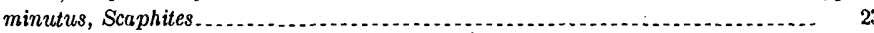

montanensis, Clioscaphites............... 2, $3,5,6,7,8,9,10,11,34,35,36,37$, pls. $16,17,20$

montanensis hesperius, Clioscaphites................................... \$4, pls. 16, 17

morrowi, Scaphites.

$2,21,22$

\section{$\mathrm{N}$}

nigricollensis, Scaphites

nigricollensis meeki, Scaphites . . ....................... 5

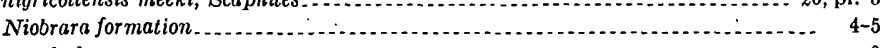

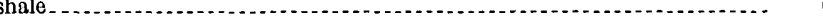

novimexicanus, Clioscaphites _.................... 2, 3, 5, 6, 7,8,9,10,11,34, s7, 39, pl. 21 Desmoscaphites.

0

obesus, Scaphites larvaeformis

oregonensis, Scaphites depressus........

$\mathbf{P}$

patulus, Scaphites

$2,3,5,6,7,20,21, \mathrm{pl} .1$

perrini, Scaphites

pisinnus, Scaphites . . ................................................ $4,7,9,25,31, \mathrm{pl}$

planus, Yezoites

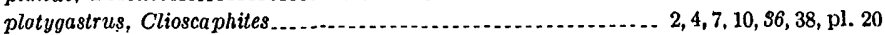

praecoquus, Scaphites .............. 2, 3, 5, 6, 7, 9, 11,20, pl.

preventricosus, Scaphites.......................... 2, 5, 6.7, 8, 9, 11, 26, 28, 29. 31, pl. 9

preventricosus artilobus, Scaphites_........................................... 87

preventricosus sweetgrassensis, Scaphites ............................... 27, 28, pl. 10

Prionocyclus macombi.

wyomingensis

pseudoaequalis, Scaphites

puerculus teshioensis, Scaphites

pygmaeus, Scaphites

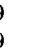

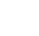


Reeside, John B., Jr., quoted.

Sage breaks member

sagensis, Scaphites.

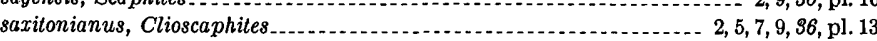
Scaphites ventricosus.

saxitonianus keytei, Clioscaphites

Scaphites aequalis

Scaphites aquilaensis

arcadiensis

auriculatus

auritus

binneyi

bosquensis.

brittonensis

carlilensis

circularis.

coloradensis

condoni.

corvensis bighornensis

delicatulus.

greenhornensis. . . .

depressus

oregonensis.

$2,3,6,7,8,9,11,32,35,36$, pl. 15

9 93, 34, pl. 15

ferronensis............. $2,5,7,9,29,24,25, \mathrm{pl} .4$

frontierensis................................................. 2, 3,9, $\$ 0, \mathrm{pl} 10$

geinitzi

hippocrepis.

hugardianus.

impendicostatus erucoides.

20

$2,3,5,7,9,10,28,32, \mathrm{pl} .11$

interjectus

$29, \mathrm{pl} .11$

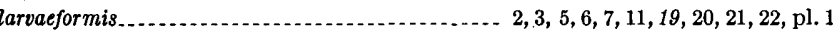

eei....

$2,3,5,6,7,11,19,20,21,22, \mathrm{pl} .1$

(10, $54, \mathrm{pl} .21$

2, 5, 7,9,28, pl. 8

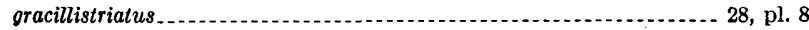

minutus.

morrowi......................................................... 2, 21,22

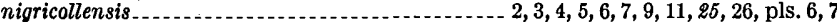

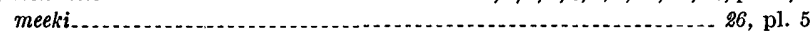

patulus.

perrini

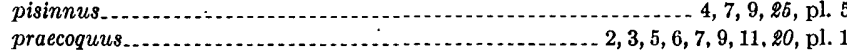

preventricosus............ 2, $5,6,7,8,9,11,26,28,29,31$, pl. 9

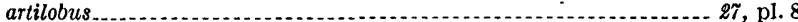

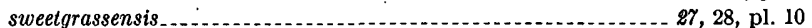

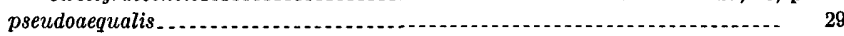

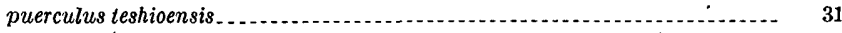

pygmaeus

sagensis_._.

subevolutus

tetonensis.............. 2, 3, 5, 7, 10, $31,32, \mathrm{pl} 14$

uintensis

ventricosus...... $2,3,5,6,7,8,9,11,27,31,32,33$, pls. 12,13

depressus

interjectus

saxitonianus

vermiformis.

veterinoous. 32,35
Scaphites aquilaensis-Continued warreni.

Page haydeni

$2,4,5,7,9,10,11,21,23,24$, pl. 3

silesiace

pl. 3

ubiquitosus

wyomingensis

$23,24,25,29$

28, pls. 3,4

whitfieldi.

wyomingensis

22,24

yonekurai.

20, 29

Sciponoceras gracile.

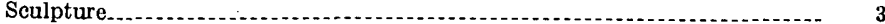

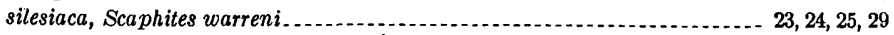

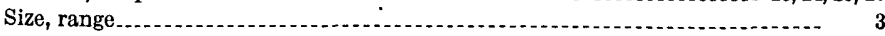

stantoni, Scaphites depressus.............. 15

subevolutus, Scaphites

Suture.

Sweetgrass arch

sweetgrassensis, Scaphites preventricosus

5

$\mathrm{T}$

teshioensis, Scaphites puerculus.

tetonensis, Scaphites.

Teranites

33

Vascoceras.................. 38,39

toolensis, Clioscaphites vermiformis............. 3, \$6, 37, pl. 19

Turner sandy member

$\mathrm{U}$

ubiquitosus, Scaphites warreni

uintensis, Scaphites

28, pls. 3,4

烈 $2,3,9,29,30$, pl. 10

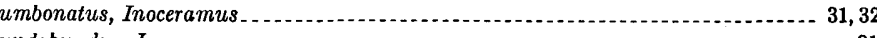

undabundus, Inoceramus

Variation

Vascoceras thomi . ventricosus, Scaphites................... 2, 3, 6, 7,8,9,11, 27.31,32,33, pls. 12, 13 ventricosus depressus, Scaphites........................................... 32 ventricosus interjectus, Scaphites ventricosus saxitonianus, Scaphites . vermiformis, Clioscaphites...................... 2, 3, 5, 7, 10,34, 35, 36, 37, 38, 39, pl. 18

Holcoscaphites.................... 35

Scaphites vermiformis toolensis, Clioscaphites .............................. veterinovus, Scaphites .................... 3

warreni, Holcoscaphites ___ Scaphites... $2,4,5,7,9,10,11,81,23,24$, pl. 3

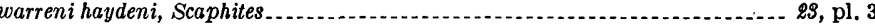
warreni silesiaca, Scaphites warreni ubiquitosus, Scaphites warreni wyomingensis, Scaphites whitfieldi, Scaphites................... 2, 3, 4, 5, 7,9, 10, 24, 25, 26, pls. 4, 5 woollgari, Collignoniceras

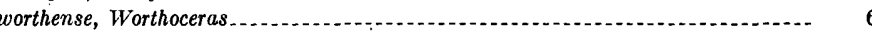
Worthoceras worthense

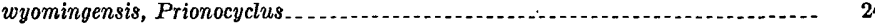

Scaphites .

wyomingensis, Scaphites warreni.

$\mathrm{Y}$

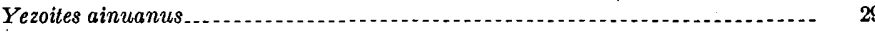

planus 29

yonekurai, Scaphites 
PLATES 1-21 


\section{PLATE 1}

[All figures natural size except as indicated on plate]

Figures 1-3. Scaphites delicatulus Warren var. greenhornensis Cobban, n. var. Bottom, top, and side views of holotype, an internal mold, U.S.N.M. 106766. From a limestone concretion 10 feet below top of Greenhorn formation at map locality 101 (p. 18).

4-15. Scaphites larvaeformis Meek and Hayden. 4-7, Third suture from last, and side, top, and bottom views of holotype, U.S.N.M. 229. From Carlile shale of eastern flank of Black Hills. 8-12, Side, front, rear, top, and bottom views of a specimen retaining most of the shell, U.S.N.M. 106743. From a limestone concretion in the basal part of Carlile shale at map locality 129 . 13, Next to last suture of an incomplete specimen, U.S.N.M. 106744 . From a limestone concretion 12 feet above base of Carlile shale at map locality 102 . 14, 15, Side and front views of a small internal mold at diameter of $4 \mathrm{~mm}$., U.S.N.M. 106745. From a limestone concretion near base of Carlile shale at map locality 137 (p. 19).

16-22. Scaphites larvaeformis var. obesus Cobban, n. var. 16-21, Side, front, rear, top, and bottom views, and last suture of holotype, U.S.N.M. 106767. From a limestone concretion near base of Carlile shale at map locality 124. 22, Cross sestion at diameter of $17 \mathrm{~mm}$. of septate whorls of an incomplete specimen, U.S.N.M. 106768, from same locality as figures 14,15 (p. 20)

23-32. Scaphites patulus Cobban, n. sp. 23-25, Bottom, top, and side views of an incomplete, small, slender, paratype, U.S.N.M. 106771, from same locality as figures 16-21. 26-31, Side, front, rear, top, and bottom views, and cross section through middle of curved part of living chamber of holotype, U.S.N.M. 106796. From a limestone concretion near base of Carlile shale at map locality 121. 32, Side view of the largest known specimen, U.S.N.M. 106770 , from same locality as figures 26-31 (p. 20).

33-36. Scaphites praecoquus Cobban, n. sp. Bottom, top, rear, and side views of holotype, U.S.N.M. 106758, from same locality as figures 14,15 (p. 20). 


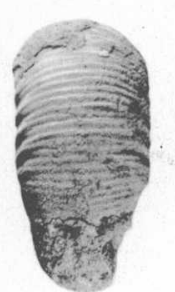

1

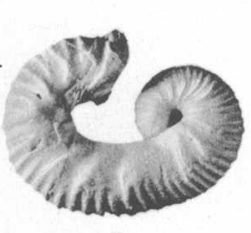

8

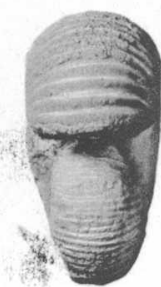

2

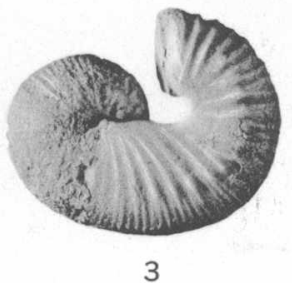

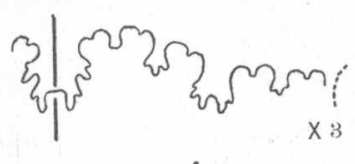
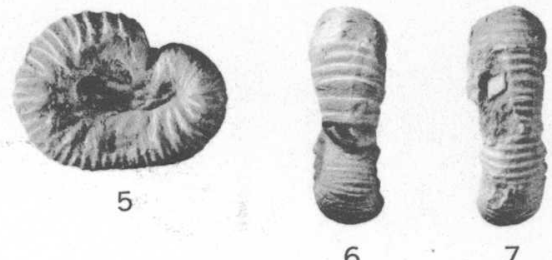

6

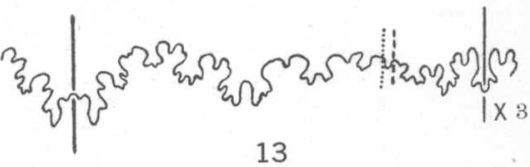

11

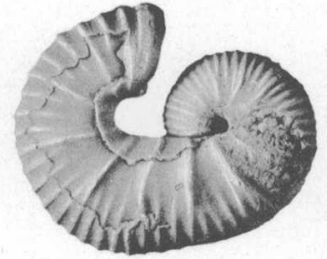

16

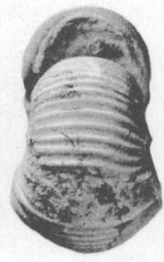

17

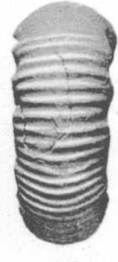

12

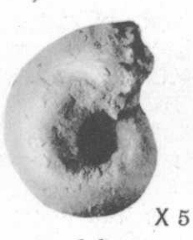

14

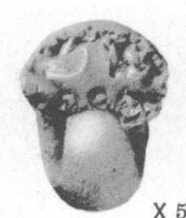

15

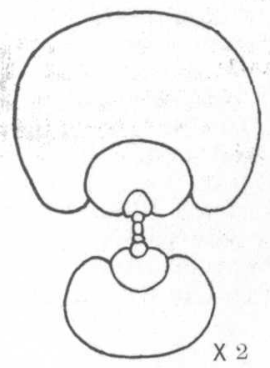

22

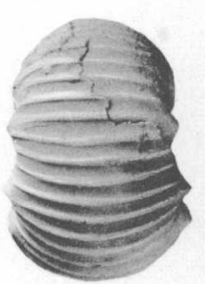

28

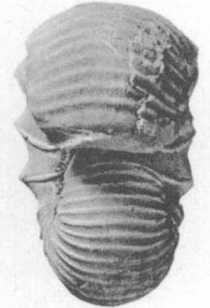

29

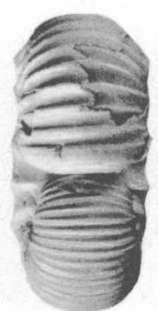

19

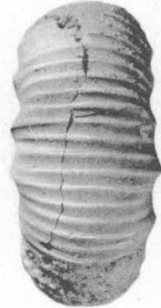

20

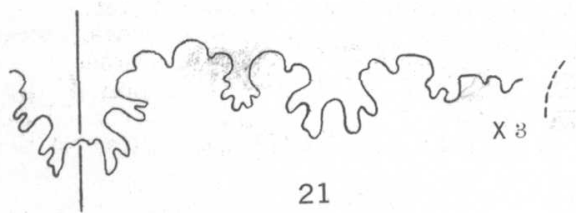

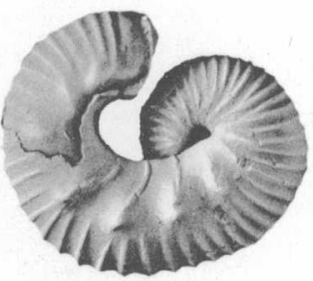

26

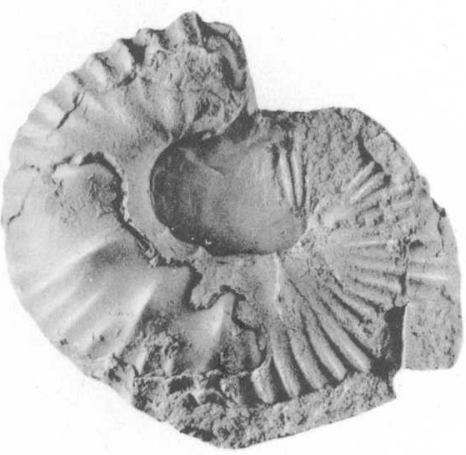

32

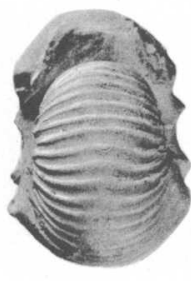

27

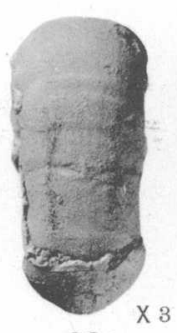

33

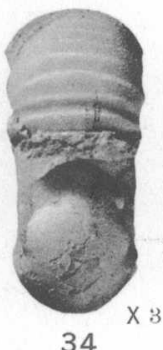

34
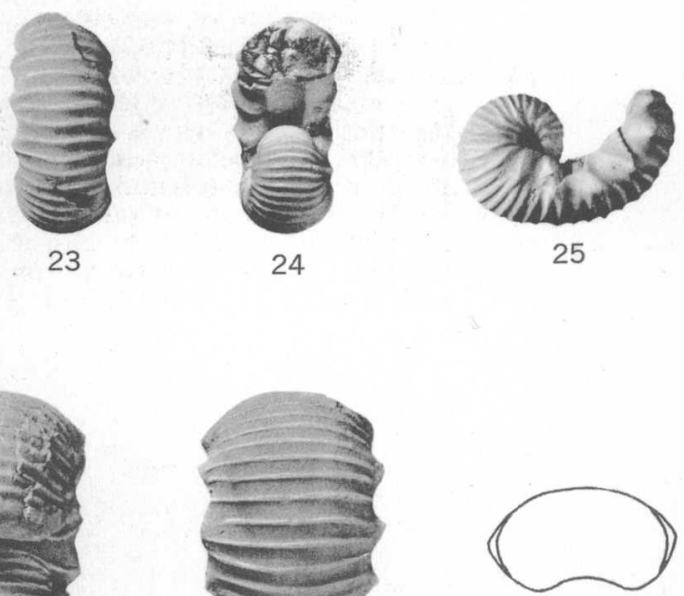

31

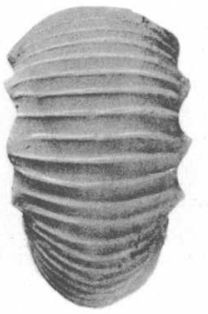

30

SCAPHITES OF THE COLORADO GROUP 


\section{PLATE 2}

[All figures natural size except as indicated on plate]

Figures 1-8. Scaphites arcadiensis Moreman. 1, 2, Side and bottom views of an incomplete living chamber, as internal mold, U.S.N.M. 106765b. From a ferruginous-concretion.bed 57-81 feet above base of Carlile shale at map locality 103 . 3 , 4t Side and rear views of an internal mold of a living chamber, U.S.N.M. 106765c, from the same locality. 5-8, Next to last sutiuc, and side, top, and bottom views of a specimen retaining part of the shell, .U.S.N.M. 106765a, from the same looality (p. 21).

9-23. Scaphites carlilensisimorrow. 9-11, Twelfth from last suture, and bottom and side views of a small, slender specimen retaining part of the shell, U.S.N.M. $106742 \mathrm{a}$, from same locality as figures $1,2.12-17$, Side; if inont, rear, top, and bottom views, and second from last suture of an internal mold retaining part of the shell; U.S.N.M. $106742 \mathrm{~b}$, from same locality as figures 1, 2. 18, Fourth from last suture of an adult specimen', U.S.N.M. 106740: From a coneretion found in the upper part of the Blue Hill shale member of the Carlile shale at map locality 268. 19-21, Fifth from last suture, and side and rear views of an internal mold of the septate whorls and beginning of the living chamber of the largest known specimen from the Black Hills, U.S.N.M. 106742c, from same locality as figures 1,2. 22, 23, Rear and side views of the internal mold of a large living chamber, U.S.N.M. 106741, from the same locality as figures 1,2 (p. 21). 

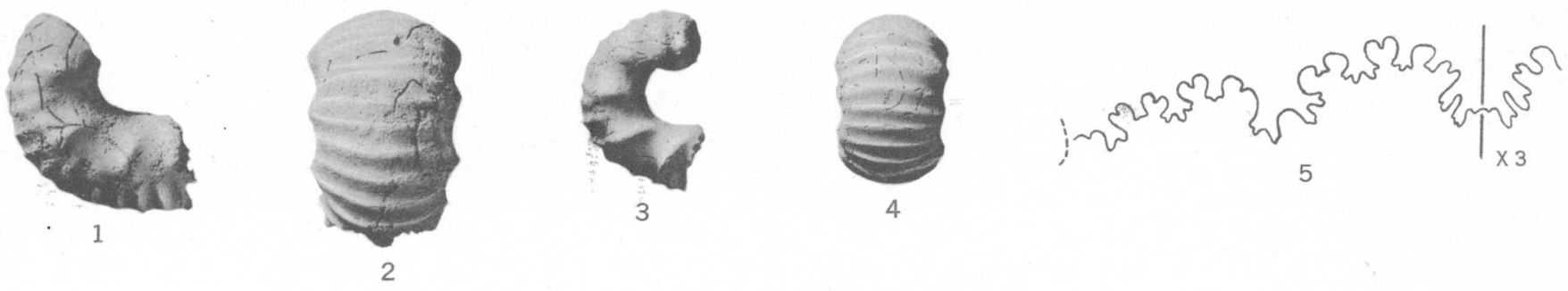

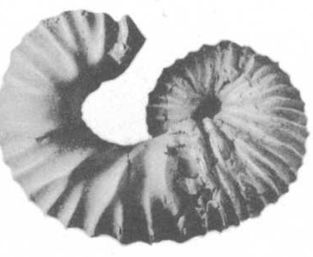

6

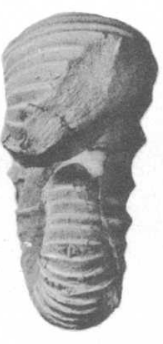

7

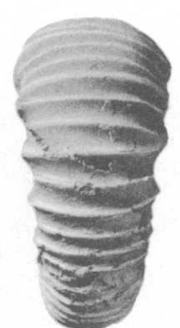

8

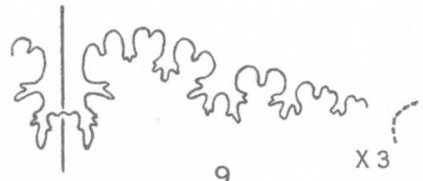

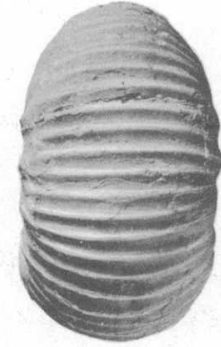

14

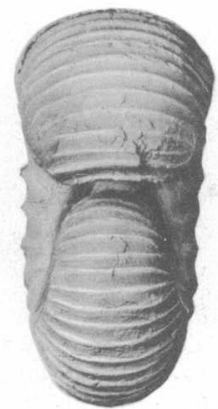

15

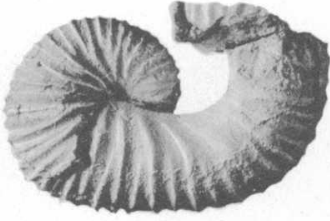

11

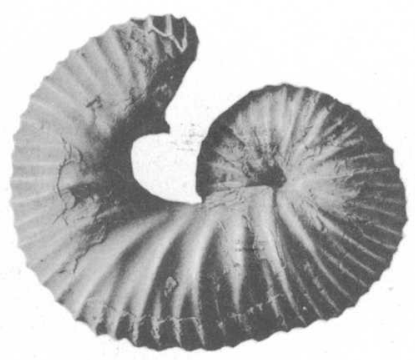

12

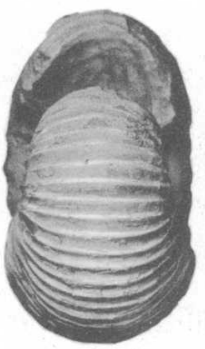

13

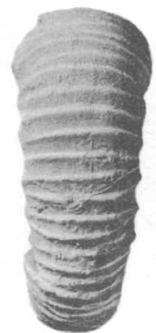

10

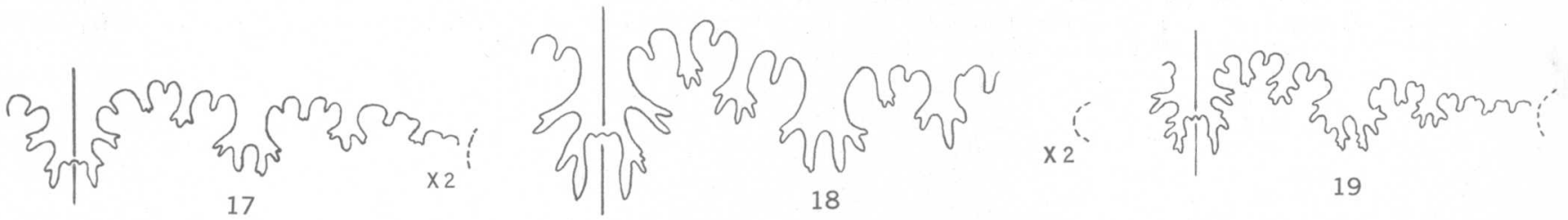

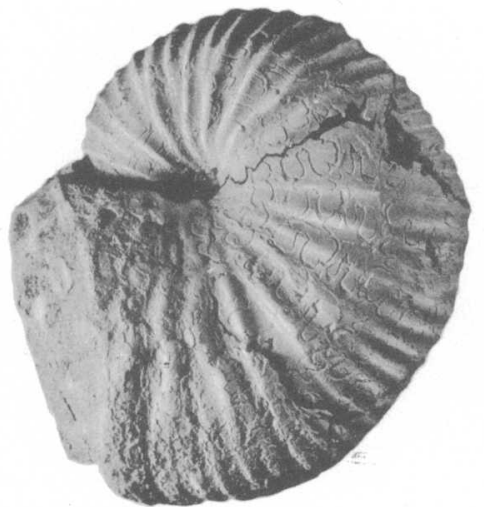

20

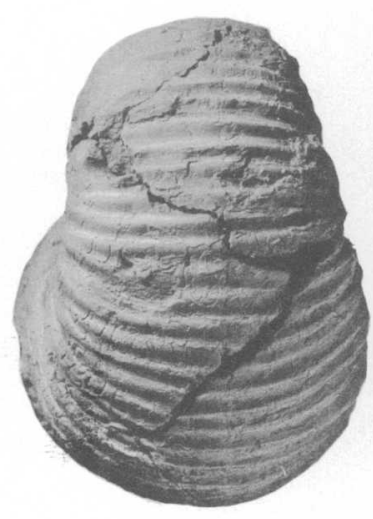

21

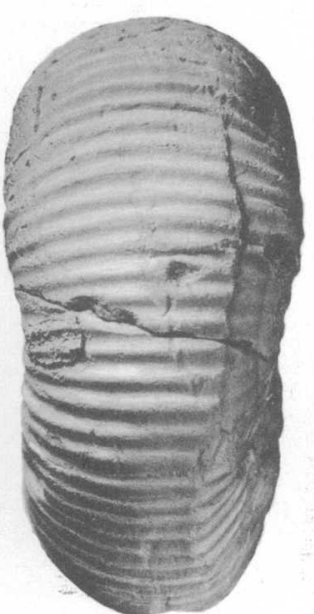

22

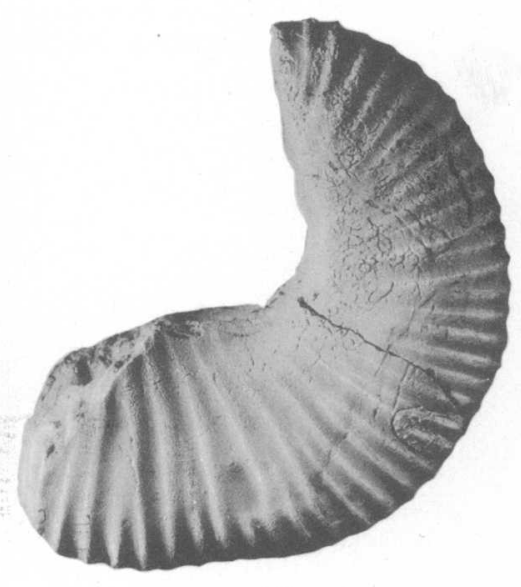

23

SCAPHITES OF THE COLORADO GROUP 


\section{PLATE 3}

[All figures natural size except as indicated on plate]

Ficures 1-7. Scaphites veterinovus Cobban, n. sp. From a calcareous concretion 14.5 feet above base of Turner sandy member of Carlile shale at map locality 108. 1-6, Side, top, and bottom views of holotype, U.S.N.M. 106763. 7, Next to last suture of paratype, U.S.N.M. 106764 (p. 23).

8-21. Scaphites warreni Meek and Hayden. 8, Second from last suture of a specimen, U.S.N.M. 106750, from a calcareous concretion 36 feet above base of Turner sandy member of Carlile shale at map locality 109. 9-12, Side, rear, top, and bottom views of a specimen, U.S.N.M. 106746, from same locality as figures 1-7. 13, Side view of holotype, U.S.N.M. 225. From the Carlile shale at map locality 125. 14, Cross section at diameter of 21.5 mm. through the septate whorls of an adult specimen, U.S.N.M. 106747. From a thin sandstone bed in the Mancos shale 150 feet below the Tocito sandstone lentil at map locality 274. 15-20, Front, rear, side, bottom, and top views, and third from last suture of an internal mold, U.S.N.M. 106748, from same locality as figure 14. 21, Fourth from last suture of a specimen, U.S.N.M. 106749, from the Frontier formation at map locality 231 (p. 21).

22-25. Scaphites warreni var. haydeni Cobban, n. var. Next to last suture, and rear, bottom, and side views of holotype, U.S.N.M. 106761. From same locality as figure 21 (p. 23).

26, 27. Scaphites warreni var. ubiquitosus Cobban, n. var. Side and rear views of an incomplete adult living chamber retaining part of the shell, U.S.N.M. 106754. Associated with the type of the species (p. 23). 

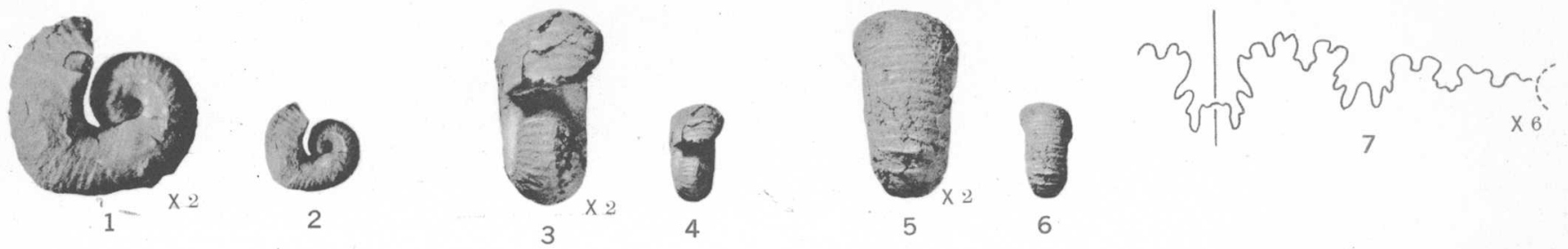
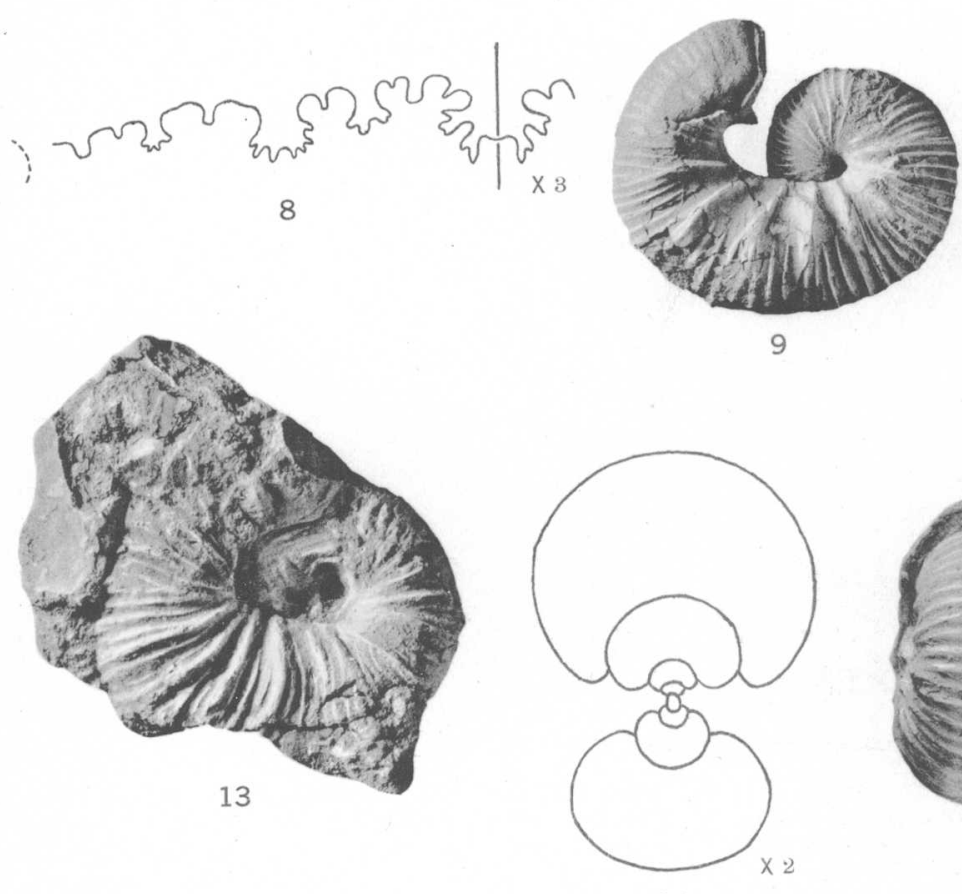

14

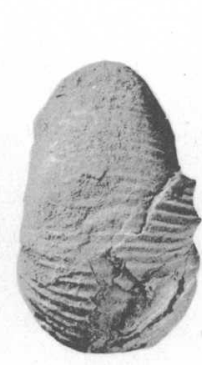

10

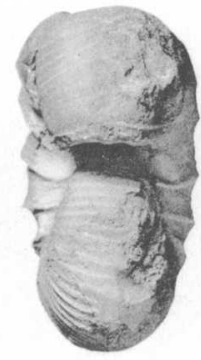

11

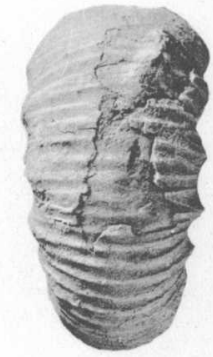

12

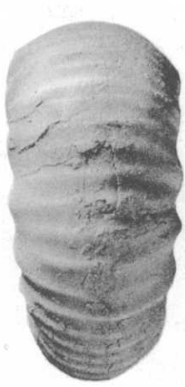

18

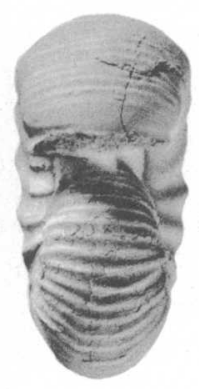

19

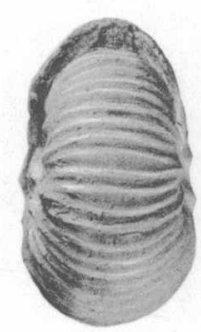

15

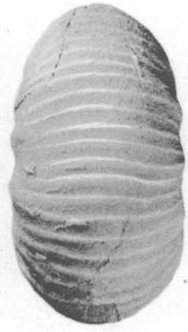

16

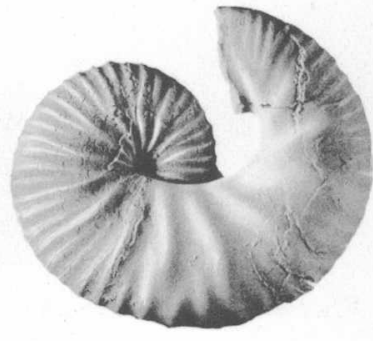

17

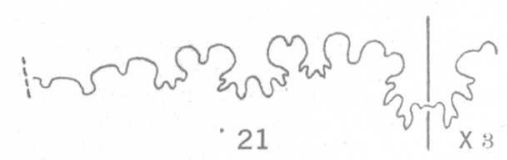

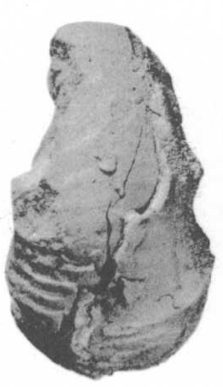

23

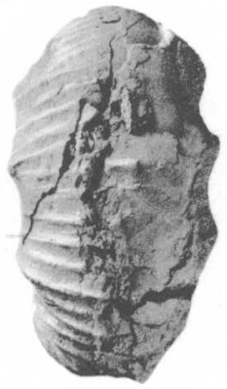

24

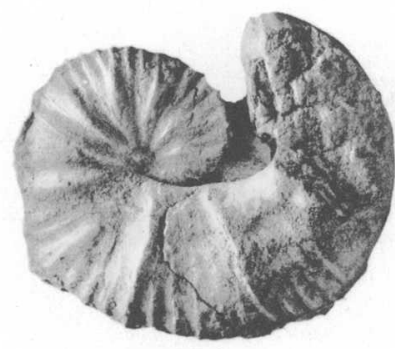

25

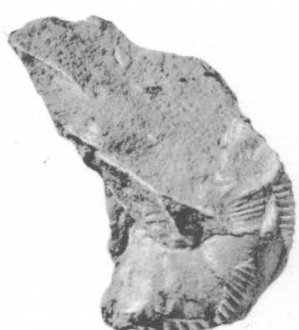

26

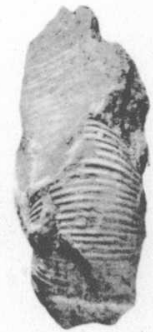

27

SCAPHITES OF THE COLORADO GROUP 


\section{PLATE 4}

[All figures natural size except as indicated on plate]

Fig UREs 1-15. Scaphites warreni var. ubiquitosus Cobban, n. var. 1-5, Side, rear, top, and bottom views, and second from last suture (composite) of holotype, an internal mold, U.S.N.M. 106751. From a thin sandstone bed in the Mancos shale about 150 feet below base of Tocito sandstone lentil at map locality 274. 6-11. Seventh from last suture, and top, bottom, rear, front, and side views of an internal mold, U.S.N.M. 106752, from same locality as figures 1-5. 12-15, Last suture, and bottom, rear, and side views of an internal mold of a small adult specimen, U.S.N.M. 106753. From the Mancos shale at map locality 273 (p. 23).

16-29. Scaphites ferronensis Cobban, n. sp. 16-19, Third from last suture, and bottom, rear, and side views of holotype, an internal mold, U.S.N.M. 106759. From the Ferron sandstone member of Mancos shale at map locality 236. 20-25, Third from last suture, and side, front, rear, top, and bottom views of a slender paratype, an internal mold retaining part of the shell, U.S.N.M. 106760a, from same locality as holotype. 26-29, Side, rear, bottom, and top views of a stout paratype, an internal mold, U.S.N.M. $106760 \mathrm{~b}$, from same locality as holotype (p. 23).

30-40. Scaphites whiffieldi Cobban, n. sp. 30-34, Fifth from last suture, and side, rear, bottom, and top views of holotype, U.S.N.M. 106735. From a ferruginous concretion bed 251-264 feet above base of Carlile shale at map locality 112 . 35-40, Side, front, rear, top, and bottom views and next to last suture of a specimen, an internal moic, U.S.N.M. 12258a, figured by Whitfield as S. wyomingensis Meek. From the Carlile shale on the western flank of the Black Hills (p. 24). 


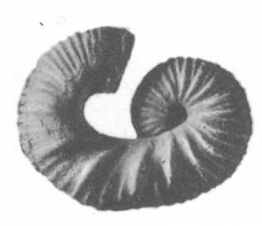

1

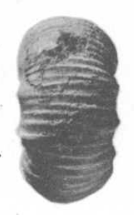

2

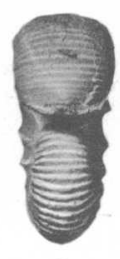

3

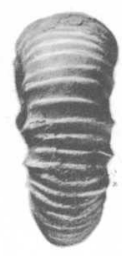

4

$$
\begin{aligned}
& \sum_{0}^{2} \int_{5}^{2} s_{20}
\end{aligned}
$$

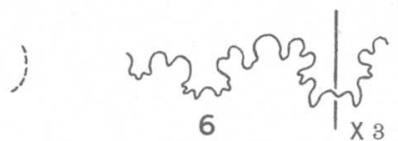

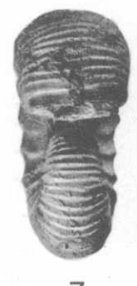

7

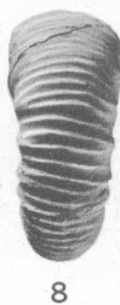

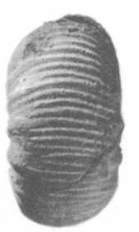

9

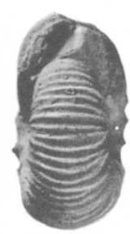

10

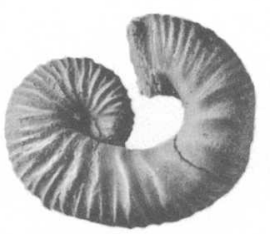

11.

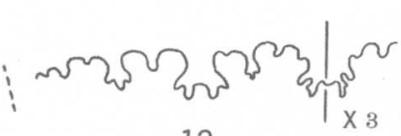
12

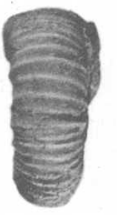

13

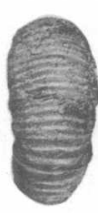

14

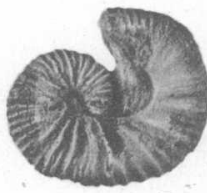

15

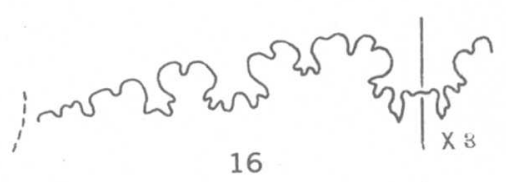

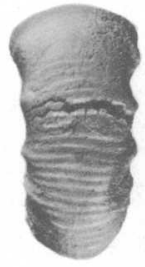

17

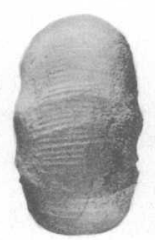

18

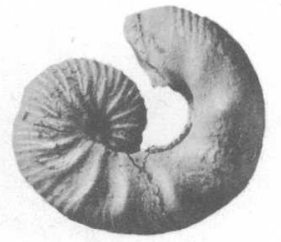

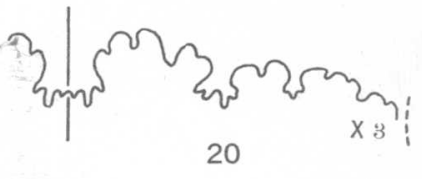

19

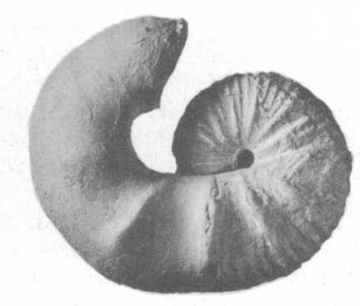

26

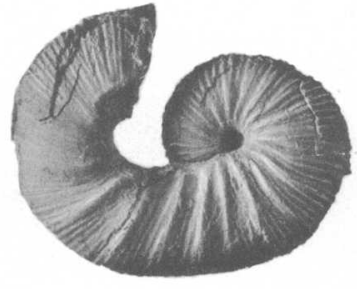

31

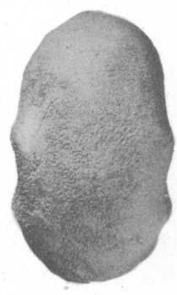

27

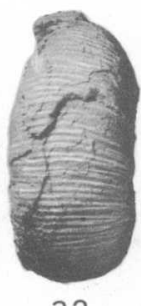

32

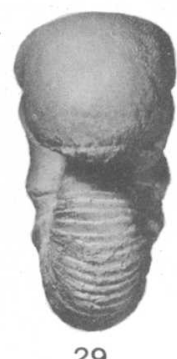

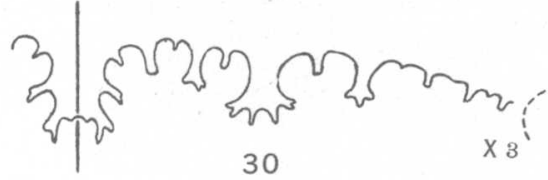

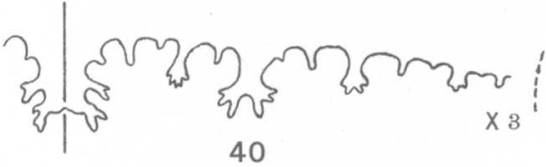

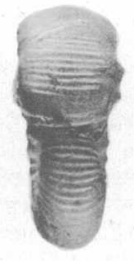

24

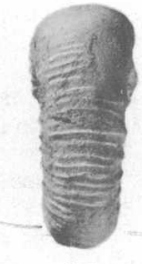

25

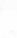

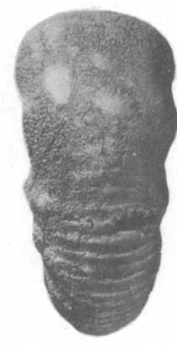

28

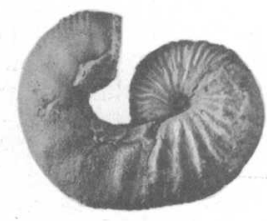

21

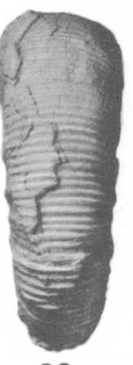

33

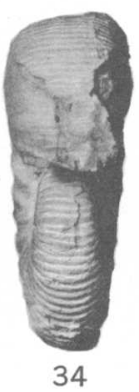

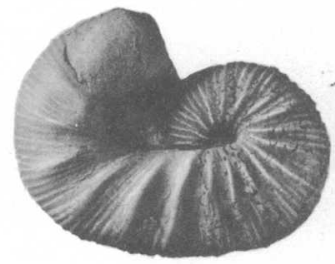

35

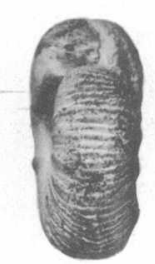

36

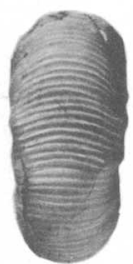

37

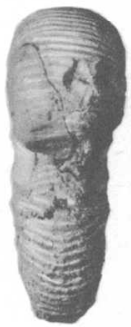

38

SCAPHITES OF THE COLORADO GROUP 


\section{PLATE 5}

[All figures natural size except as indicated on plate]

Figurns 1-4. Scaphites whitfieldi Cobban, n. sp. 1, Fragment, retaining most of the shell, from the straight part of the living chamber of the largest known specimen, U.S.N.M. 106738a. From a ferruginous concretion bed 251-264 feet above base of Carlile shale at map locality 112. 2, Cross section through the septate whorls at diameter of $16.5 \mathrm{~mm}$. of an incomplete specimen, U.S.N.M. 106737. From a calcareous concretion 43 feet above base of Turner sandy member of Carlile shale at map locality 126. 3, Second from last suture of an internal mold, U.S.N.M. 106736. From a ferruginous concretion in Turner sandy member of Carlile shale at map locality 136. 4, Fragment of a sandy, ferruginous concretion containing numerous specimens of S. whitfieldi, U.S.N.M. $106738 \mathrm{~b}$, from same locality as figure 1 (p. 24).

5-8. Scaphites pisinnus Cobban, n. sp. Bottom and side views, and sixth from last suture of holotype, an internal mold retaining part of the shell, U.S.N.M. 106762. From same locality as figure 1 (p. 25).

9-26. Scaphites nigricollensis var. meeki Cobban, n. var. From a calcareous concretion bed 59 feet below top of Turner sandy member of Carlile shale at map locality 114. 9, Cross section through septate whorls at diameter of $16.5 \mathrm{~mm}$. of a complete specimen, U.S.N.M. 106734b. 10-14, Second from last suture, and bottom, top, rear, and side views of holotype, U.S.N.M. 106733. 15-20, Side, front, rear, top, and bottom views, and next to last suture of an internal mold, U.S.N.M. 106734a. 21, Second from last suture of an internal mold of an adult specimen, U.S.N.M. 106734d. 22-26, Bottom, top, rear, and side views, and next to last suture of a large specimen retaining some of the shell, U.S.N.M. 106734c (p. 26). 

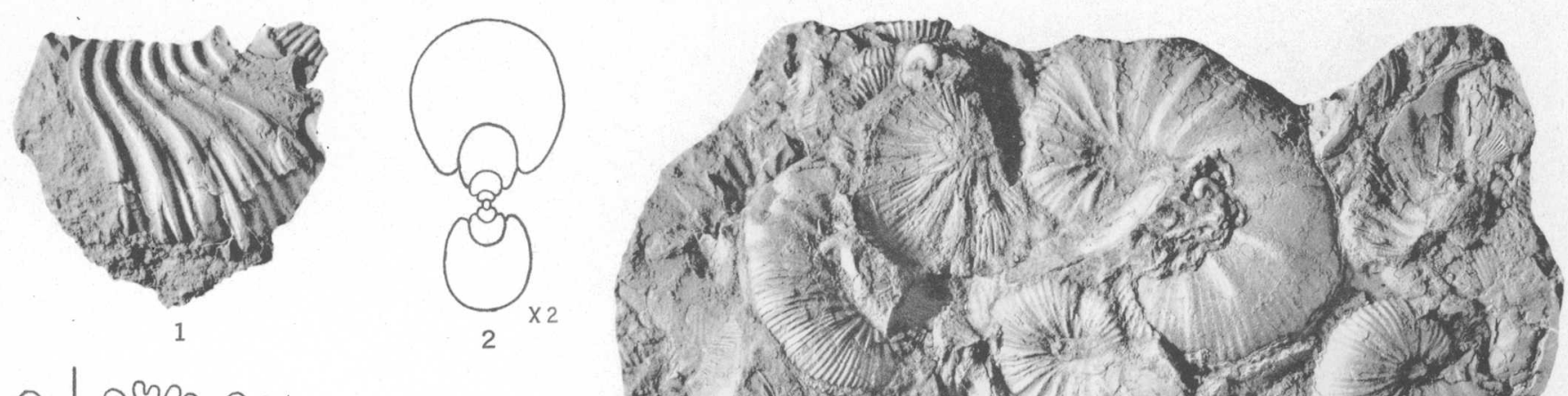

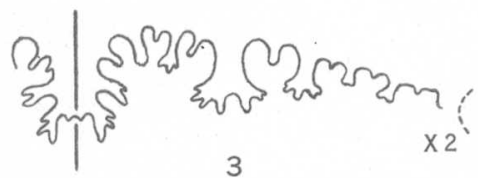

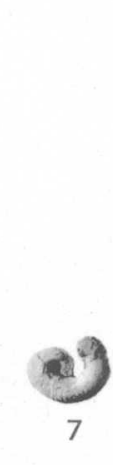

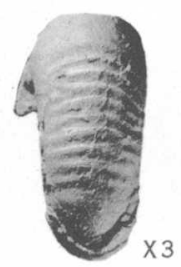

5

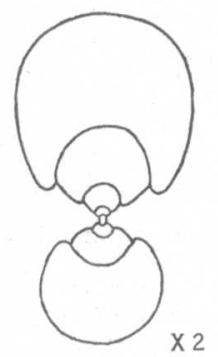

I)
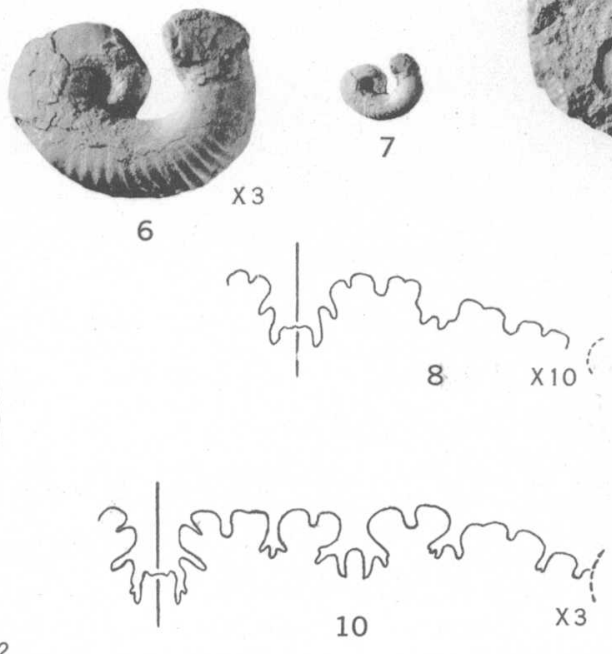

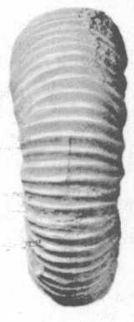

11

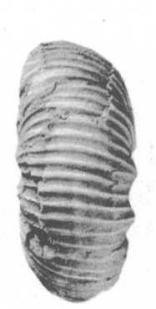

17

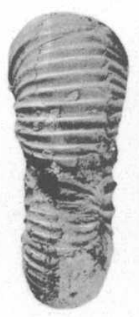

18

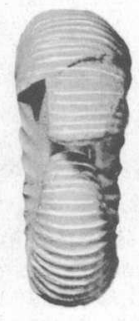

12

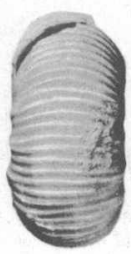

13

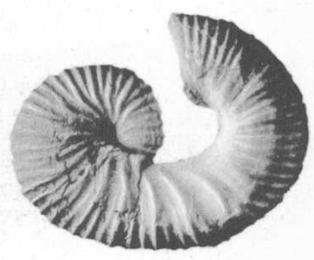

14

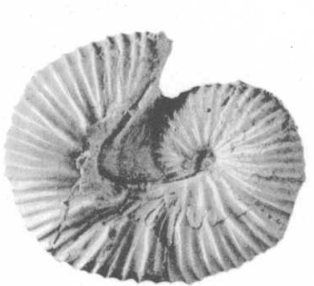

15

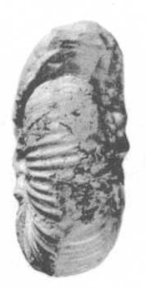

16

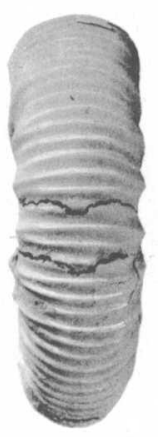

22

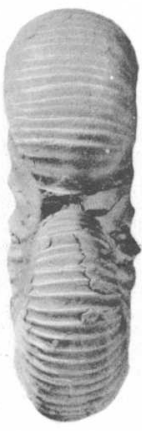

23

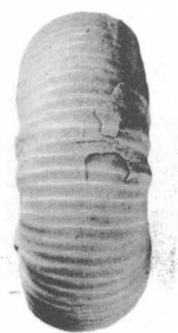

24

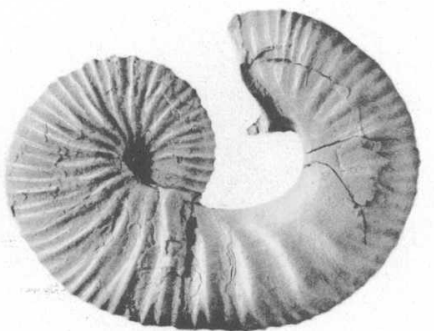

25

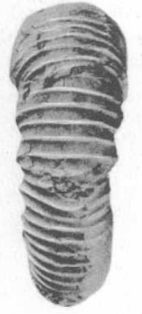

$3 \int_{20}^{202}$

19

तो

SCAPHITES OF THE COLORADO GROUP 


\section{PLATE 6}

[All figures natural size except as indicated on plate]

Figures 1-17. Scaphites nigricollensis Cobban, n. sp. From a bed of calcareous concretions 59 feet below top of Turner sandy member of Carlile shale at map locality 114 . 1-6, Bottom, top, rear, front, and side views, and second from las suture of holotype, an internal mold, U.S.N.M. 106730. 7-12, Next to last suture, and front, rear, top, bottom, and side views of a paraty pe, an internal mold, U.S.N.M. 106731b. 13-17, Fif th from last suture, and side, rear, top and bottom views of a paratype, an internal mold, U.S.N.M. 106731a (p. 25). 

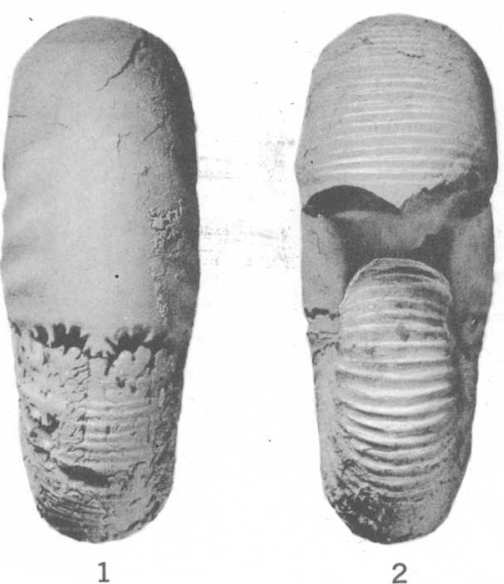

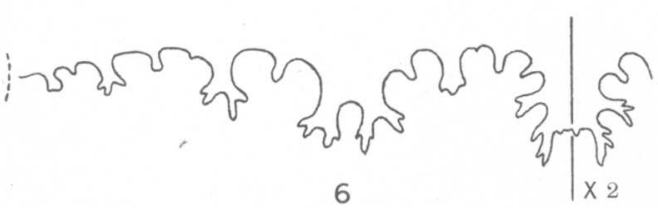

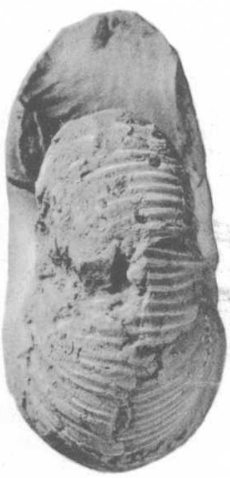

8

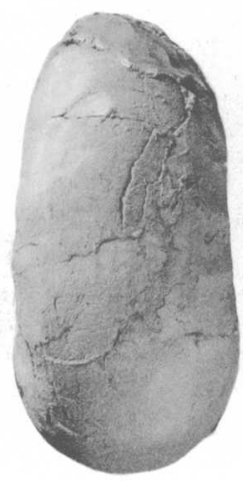

9

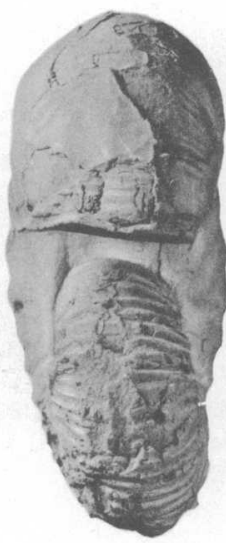

10
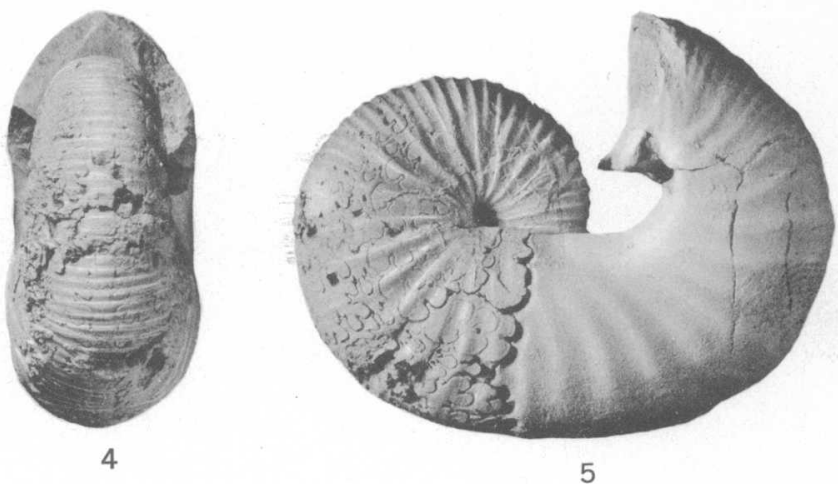

5

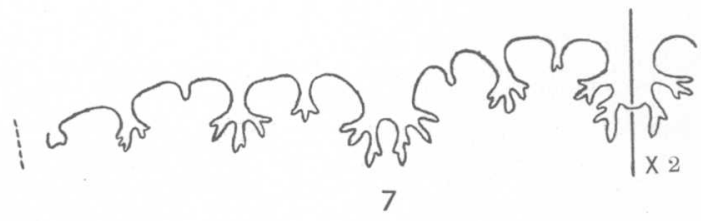

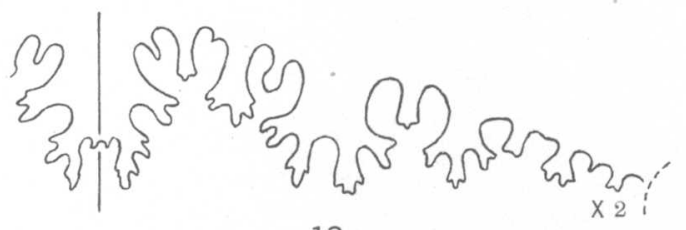

13

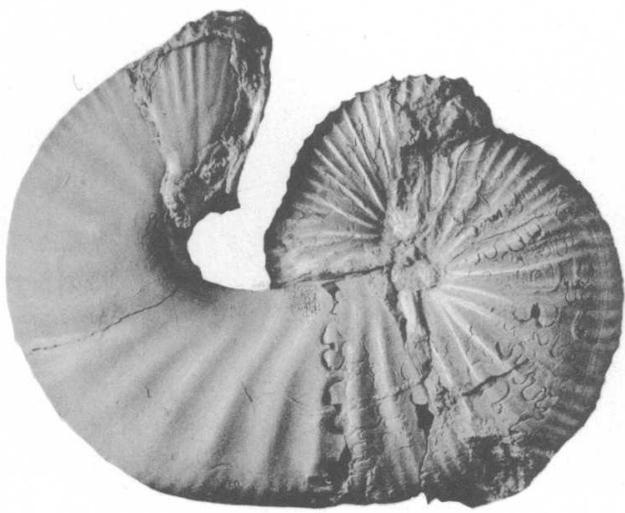

14

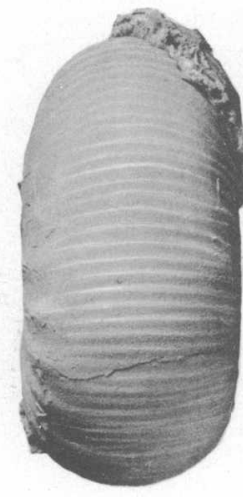

15

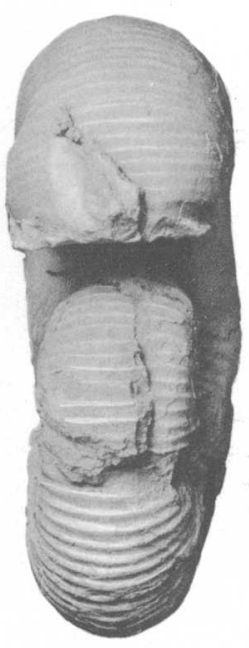

16

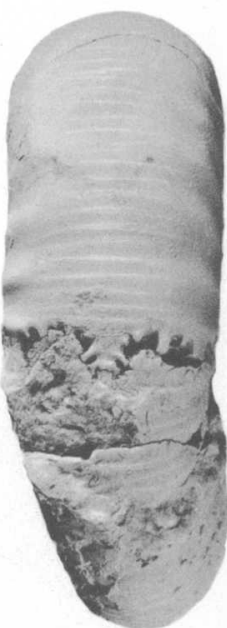

17

SCAPHITES OF THE COLORADO GROUP 


\section{PLATE 7}

[All figures natural size except as indicated on plate]

Fig URes 1-5. Scaphites nigricollensis Cobban, n. sp. 1-3, Bottom and side views, and second from last suture of an internal mold U.S.N.M. 106732: From Carlile shale at map locality 133. 4, About tenth from last suture of a paraty pe; U.S.N.M. 106731d: From a calcareous concretion $-59^{\wedge}$ feet below top of Turner sandy member-of -Carlile'shale at map tocality 114. 5, Cross section of septate whóples of an incomplete specimen, U.S.N.M. 106731c, from same locality as figure 4 (p. 25).

6-10. Scaphites corvensis Cobban, n. sp. Side, rear, and bottom views, cross sectionsthrough straight part of living chamber, and next to last suture of holotype, an internal mold retaining part of the shell, U.S.N.M. 106755. From a calcareous concretion 180 feet above base of Carlile member of Cody shale at map locality 95 (p. 26).

11-17. Scaphites corvensis var. bighornensis Cobban, n. var. Next to läst suture, side, front, rear, top, and bottom views, and cross section through straight part of living chamber of holotype, an internal mold, U.S.N.M 106756. From same locality as figures 6-10 (p. 26). 


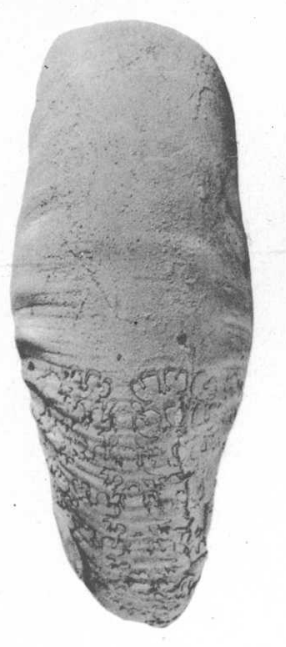

1

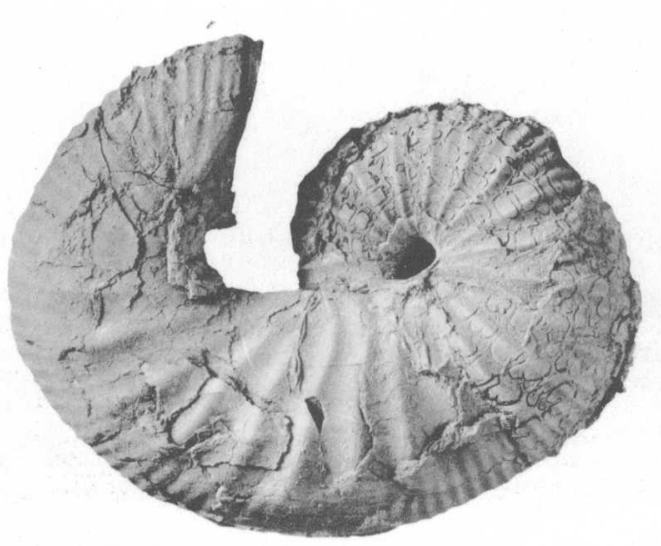

6

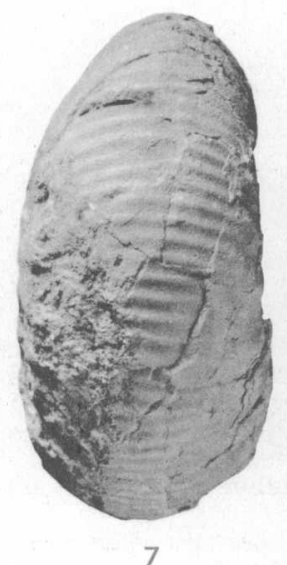

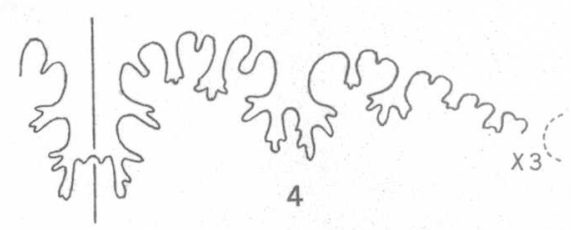

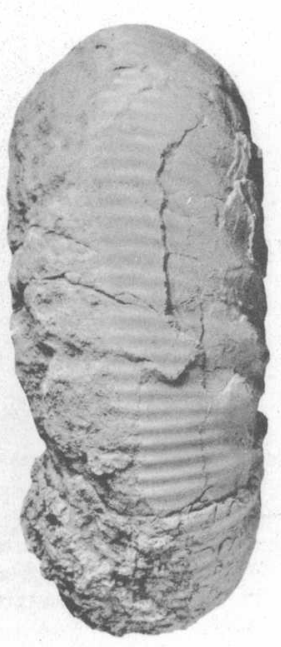

8
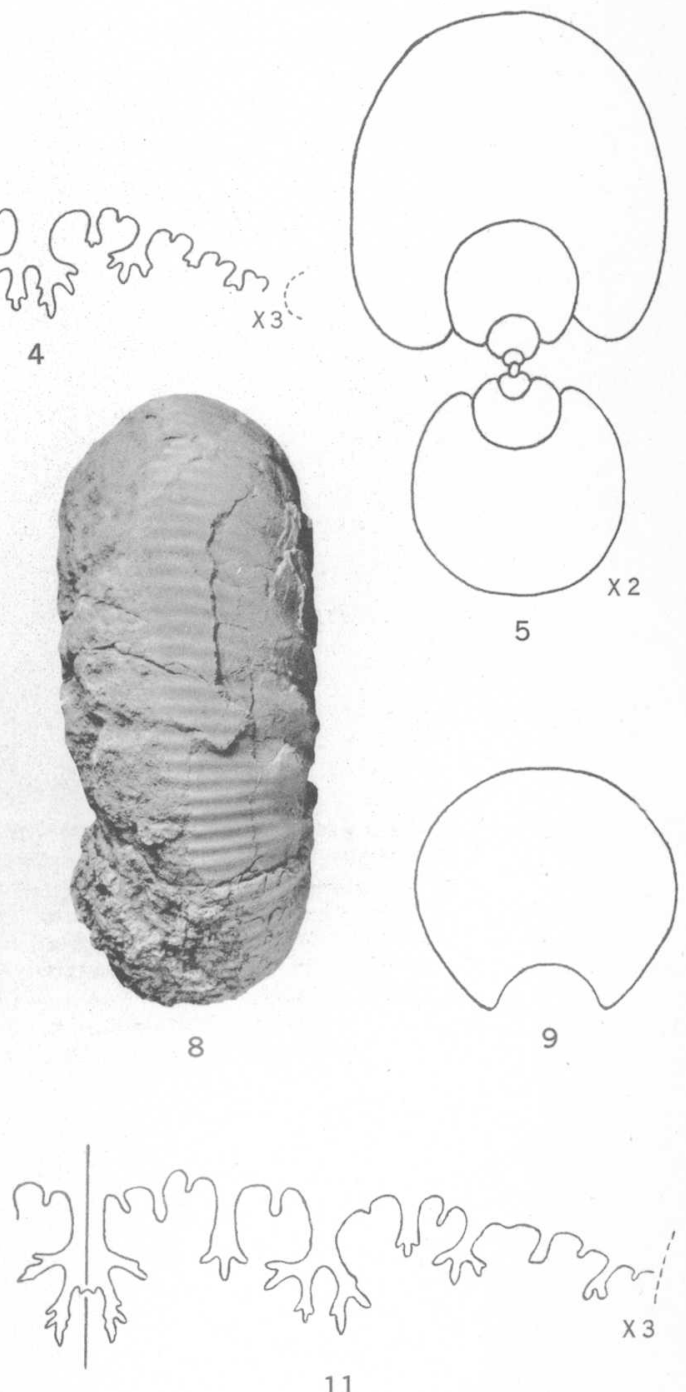

11

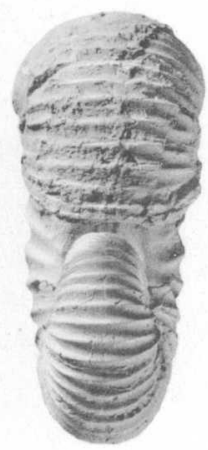

15
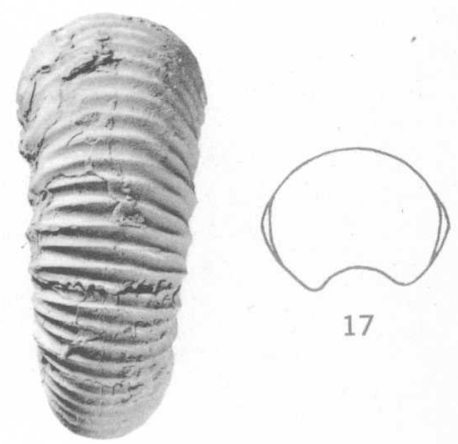

17

SCAPHITES OF THE COLORADO GROUP 


\section{PLATE 8}

[All figures natural size except as indicated on plate]

Figures 1-6. Scaphites preventricosus Cobban var. artitobus Cobban, n. var. Bottom, top, rear, front, and side views, and next to last suture (composite) of holotype, U.S.N.M. 106680. From a ferruginous concretion bed 617-634 feet'below top of Colorado shale at map locality 34 (p. 27).

7-13. Scaphites mariasensis Cobban var. gracillistriatus Cobban, n, var. 7-11, Side, rear, bottom, and top views, and second from last suture of holotype, an internal mold, U.S.N.M. 106682. From same locality as figures 1-6. 12, 13, Side and front views of an immature specimen with nearly complete living chamber, U.S.N.M. 106683, from the same locality (p. 28).

14-17. Scaphites mariasensis Cobban, n. sp. Last suture, and bottom, rear, and side views of holotype, an internal mold, U.S.N.M. 106681. From same locality as figures 1-6 (p. 28). 


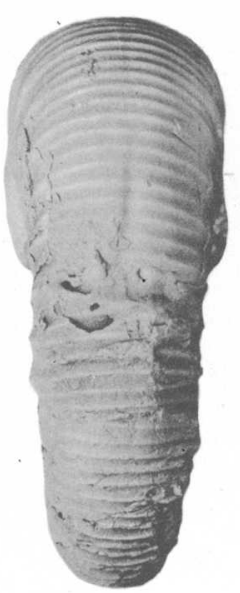

1

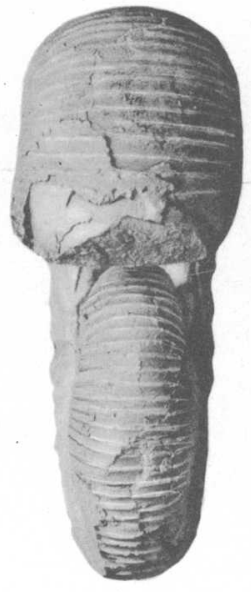

2

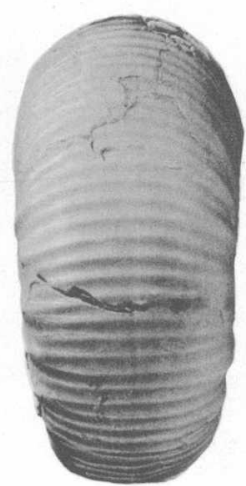

3

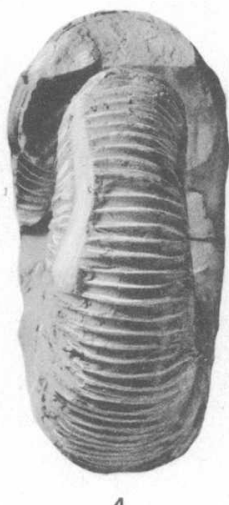

4

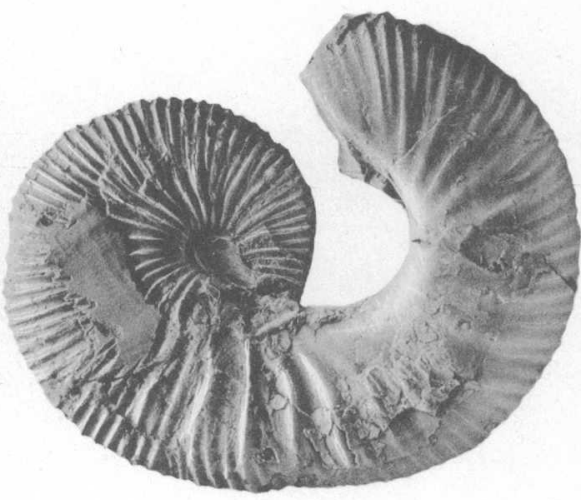

5

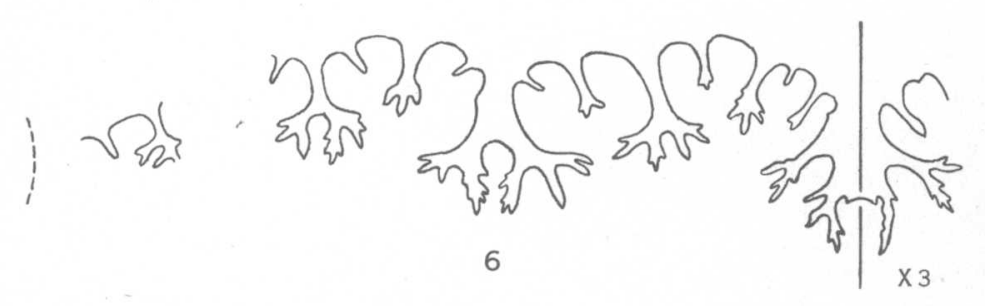

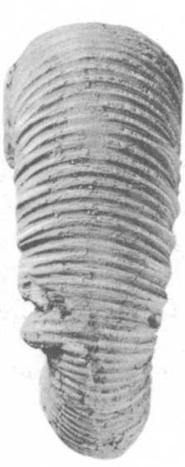

9

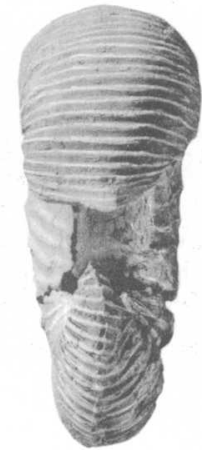

10

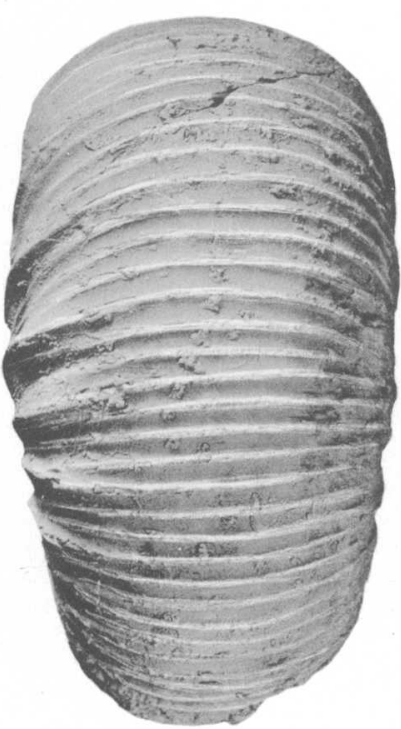

15

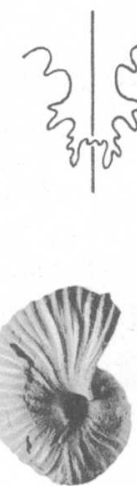

12

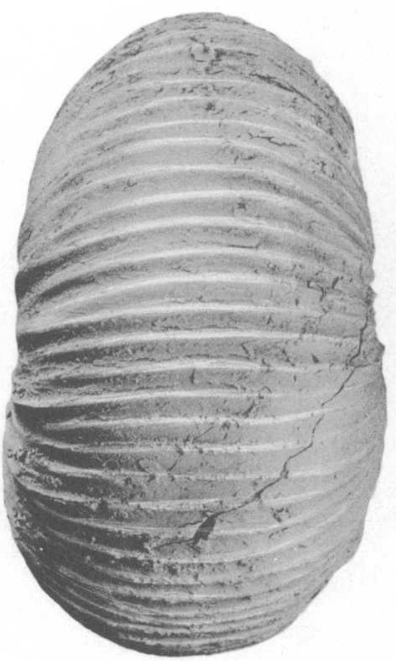

16

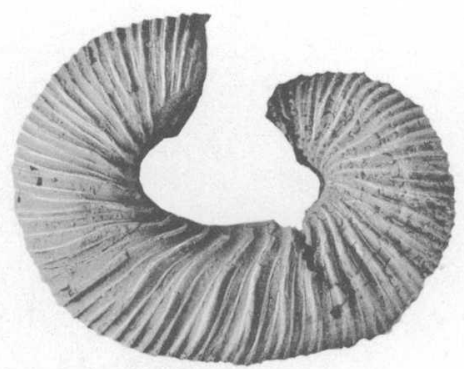

7

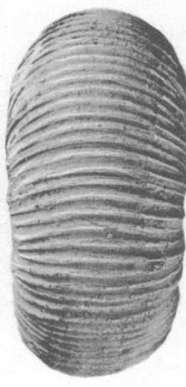

8

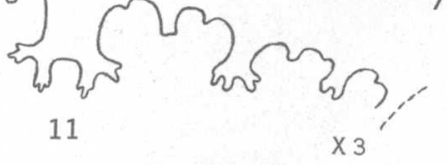

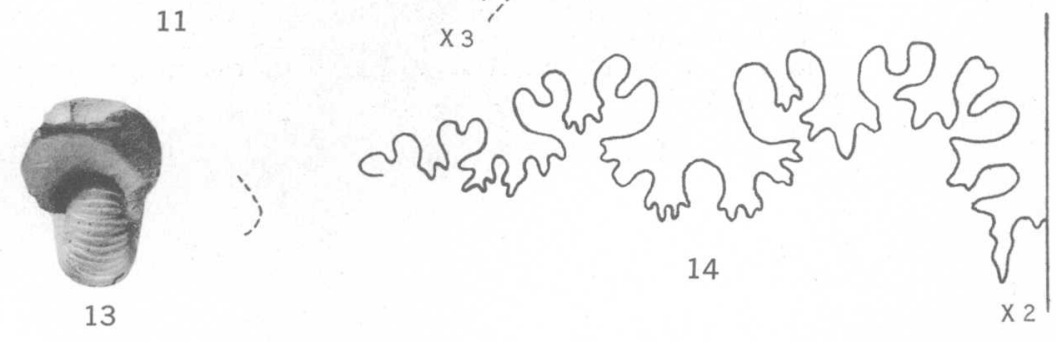

SCAPHITES OF THE COLORADO GROUP 


\section{PLATE 9}

[All figures natural size except as indicated on plate]

Frgures 1-16. Scaphites preventricosus Cobban, n. sp. 1-4, Side and front views of internal whorls at diameter of $11.8 \mathrm{~mm}$. of a specimen retaining most of the shell, U.S.N.M. 106676d. From a bed of calcareous concretions 514-525 feet below top of Colorado shale at map locality 35. 5, Side view of the earliest whorls of a specimen at diameter of $2.8 \mathrm{~mm}$., U.S.N.M. $106676 \mathrm{a}$, from the same"locality. 6-8, Front and side views, and last suture of an immature specimen with most of the living chamber, U.S.N.M. $106676 \mathrm{c}$, from the same locality. 9, Cross section through septate whorls at diameter of $32.5 \mathrm{~mm}$. of an incomplete specimen, U.S.N.M. 106679. From a bed of calcareous concretions in the Colorado shale 202-207 feet above top of a calcareous member of Greenhorn age at map locality 48. 10, Cross section at diameter of $4 \mathrm{~mm}$. of an immature specimen, U.S.N.M. $106676 \mathrm{~b}$, from same locality as figures 1-4. 11-16, Sixth from last suture, and front, rear, side, top, and bottom views of holotype, an internal mold, U.S.N.M. 106675, from same locality as figures 1-4 (p. 26). 


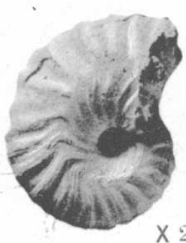

1 .

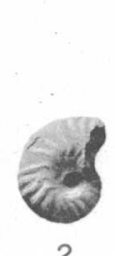

2

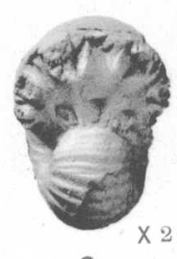

3

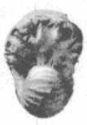

4
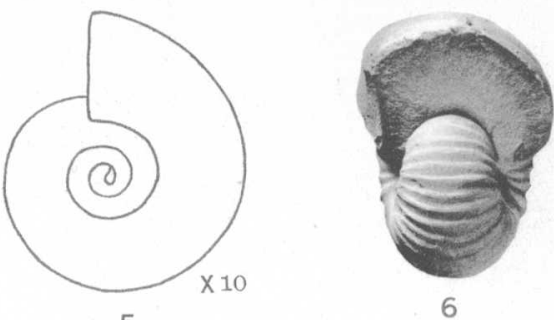

6

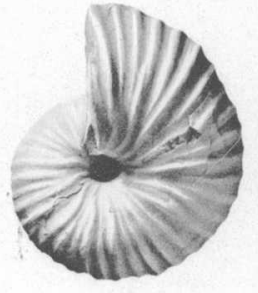

7

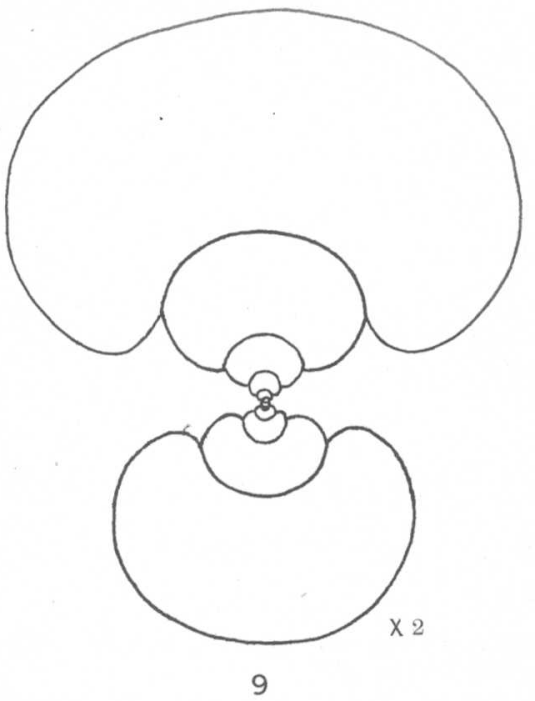

(2) nas?

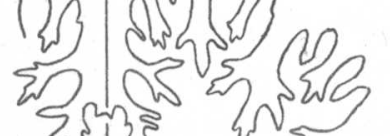

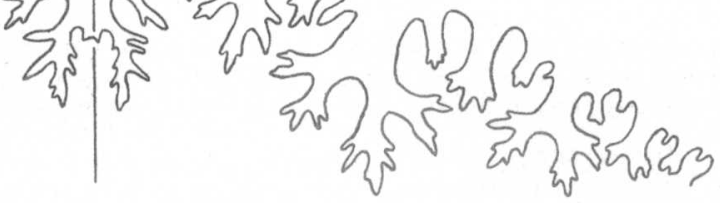

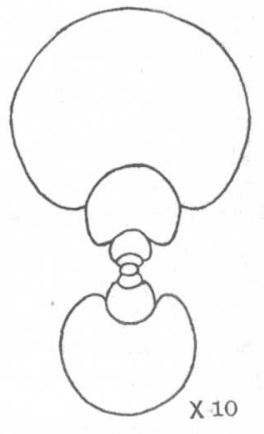

. रूजी

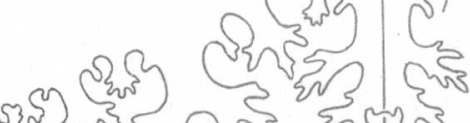

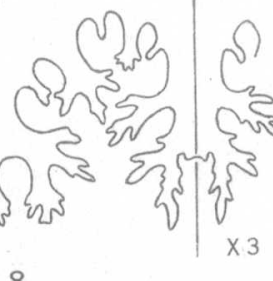

11 $x+i^{\cdots}$

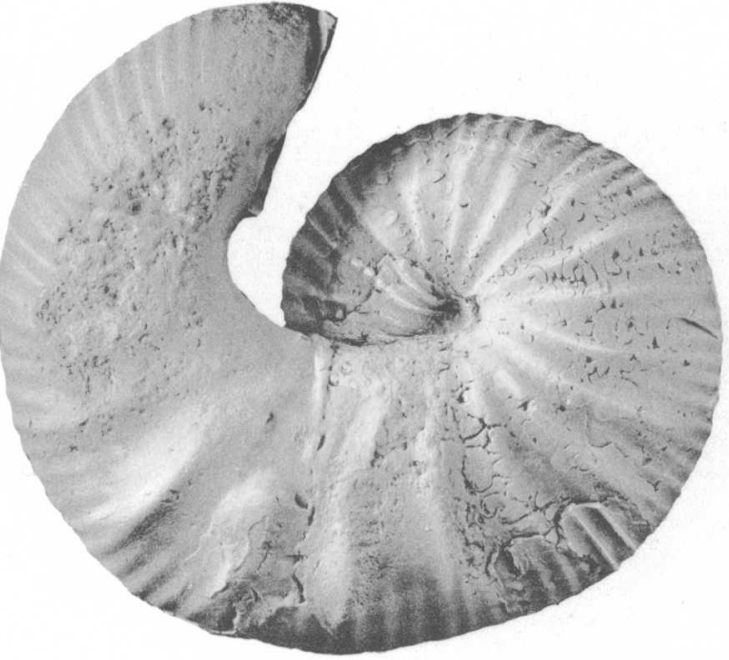

14

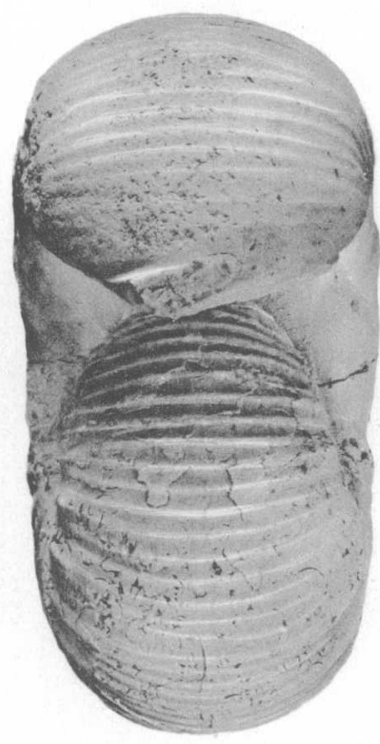

15

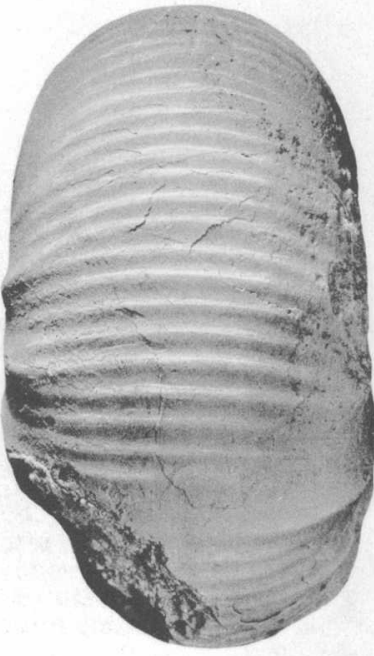

13

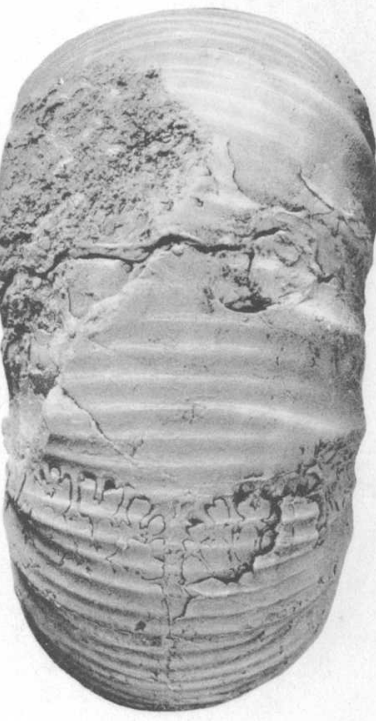

16

SCAPHITES OF THE COLORADO GROUP 


\section{PLATE 10}

[All figures natural size except as indicated on plate]

Figures 1-4. Scaphites frontierensis Cobban, n. sp. Side, rear, and bottom views of holotype, an internal mold, U.S.N.M. 106704. From a sandstone bed 180 feet below top of Frontier formation at map locality 180 (p. 30)

5, 6. Scaphites uintensis Cobban, n. sp. 5, First lateral lobe and part of adjoining saddles of last suture of paratype U.S.N.M. 106703. From the Mancos shale 180 feet above top of Frontier sandstone member at map locality 248 . 6, Side view of an artificial cast made from the holotype, an impression in shale, U.S.N.M. 106702, from same locality as figure 5 (p. 29).

7-10. Scaphites sagensis Cobban, n. sp. 7, 8, Bottom and side views of holotype, a distorted specimen retaining part of the shell, U.S.N.M. 106696. From upper part of Frontier formation at map locality 195. 9, 10, Rear and side views of the internal mold of an incomplete living chamber, U.S.N.M. 106697. From upper part of Frontier formation at map locality 196 (p. 30).

11-17. Scaphites auricutatus Cobban, n. sp. 11-16, Side, rear, bottom, and top views, and last suture of holotype, an internal mold retaining part of the shell, U.S.N.M. 106684. From a bed of calcareous concretions 514-525 feet below top of Colorado shale at map locality 35. 17, Cross section through the septate whorls and living chamber of an adult specimen, U.S.N.M. 106685, from the same locality (p. 30)

18-25. Scaphites preventricosus Cobban var. sweetgrassensis Cobban. 18-20. Side and bottom views, and fourth from last suture of a small internal mold retaining some of the shell, U.S.N.M. 106678, from same locality as figures 11-16. 21-25, Second from last suture. and side, front, top, and bottom views of holotype, an internal mold, U.S.N.M. 106677 , from the same locality (p. 27). 

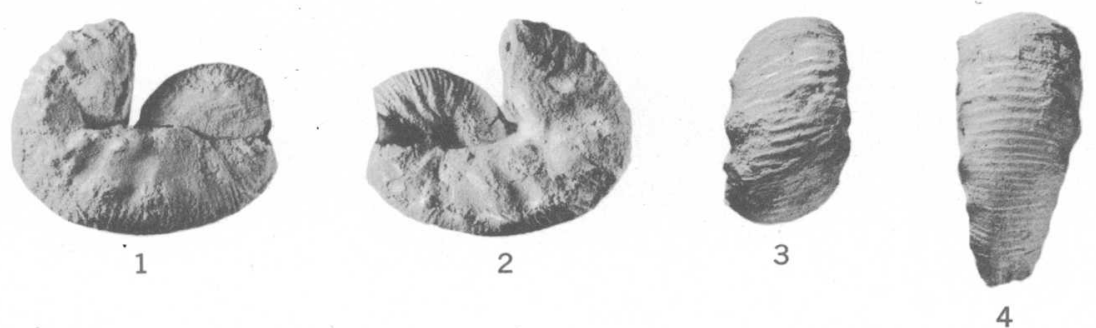

; $255^{2} \times 4$
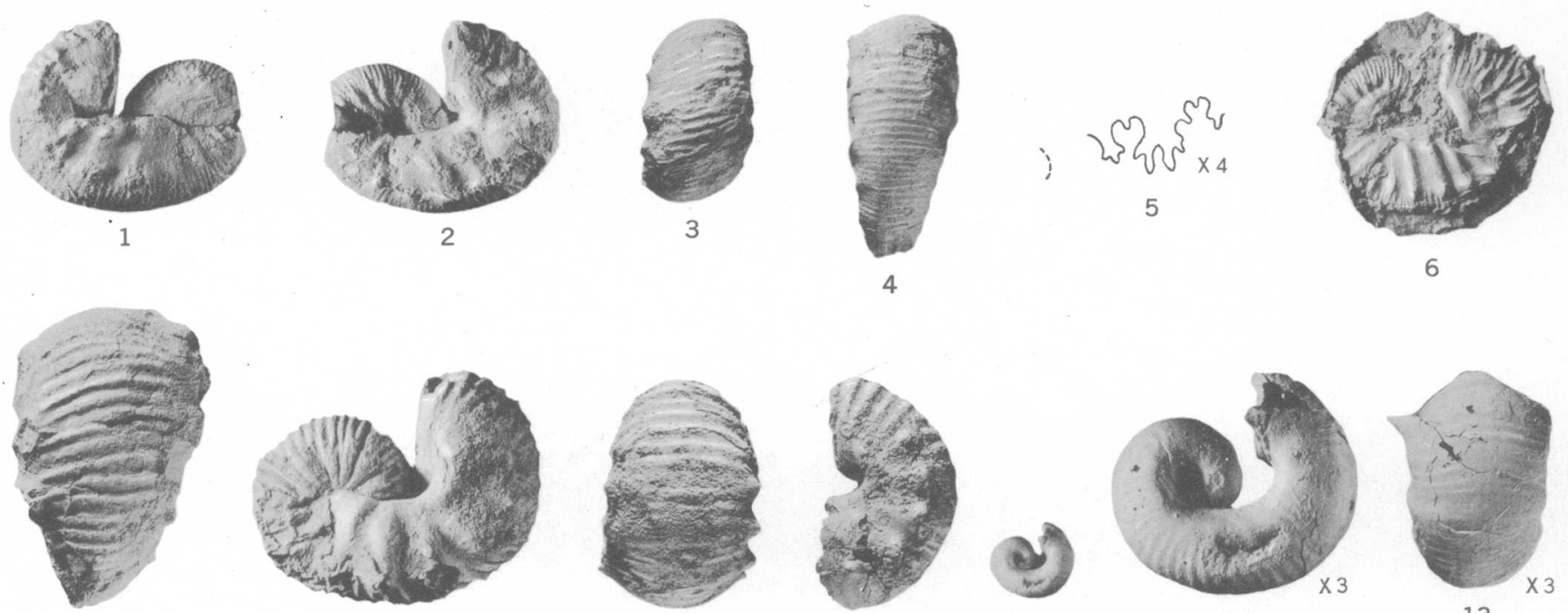

8

10

11

12
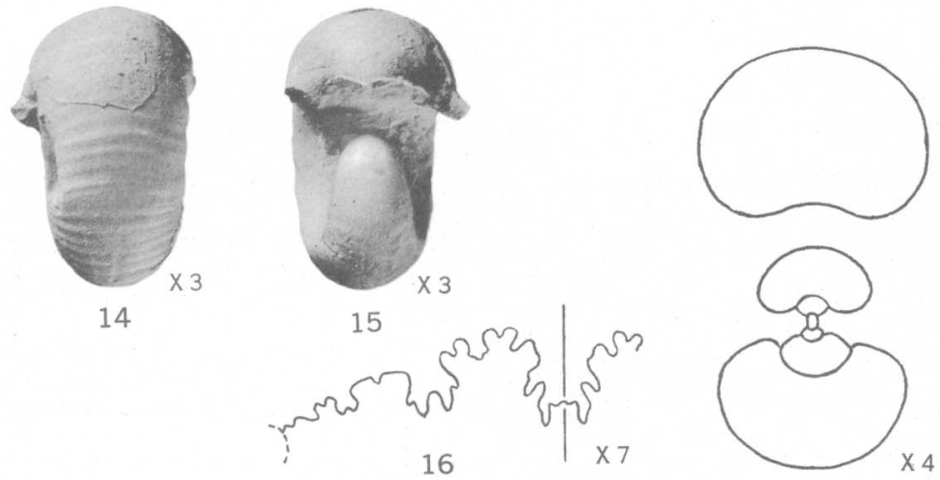

(3)
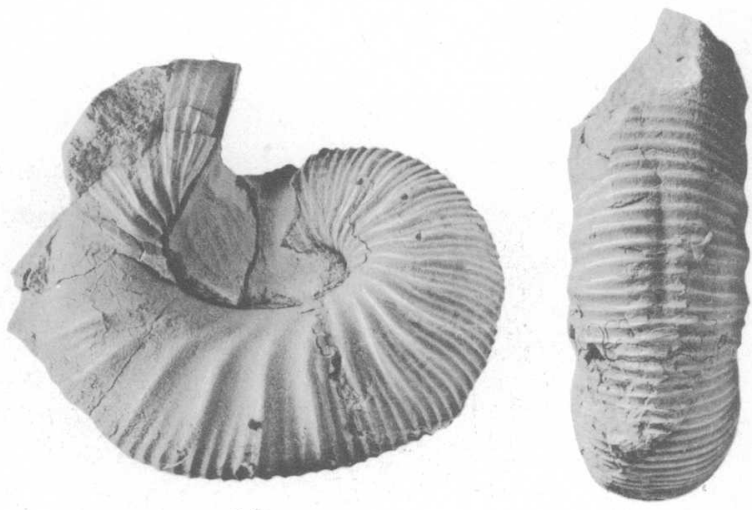

18

19

की

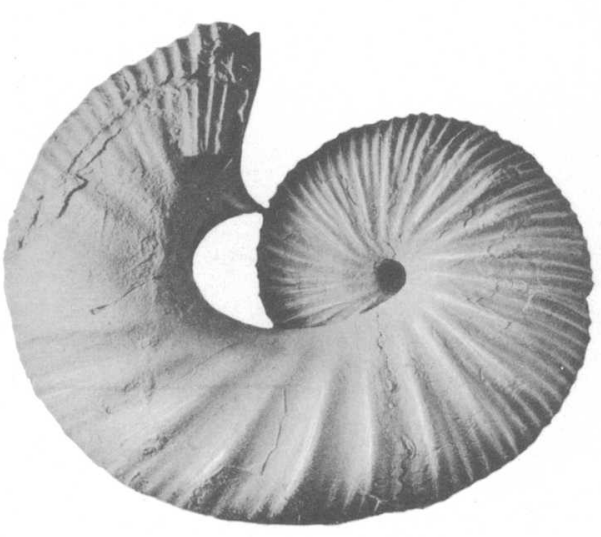

22

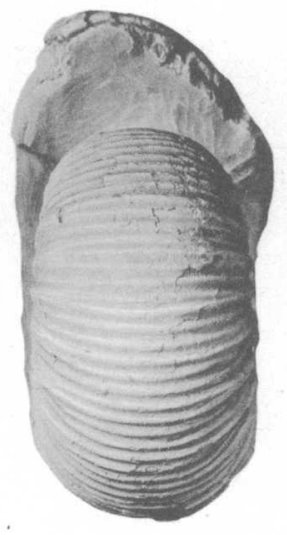

23

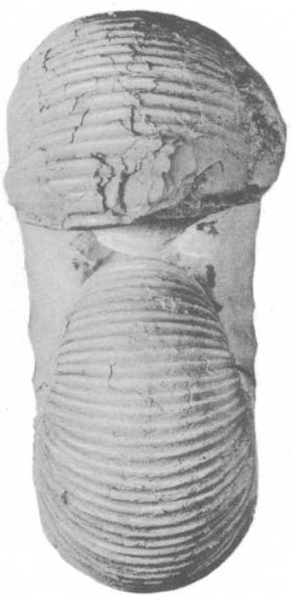

24

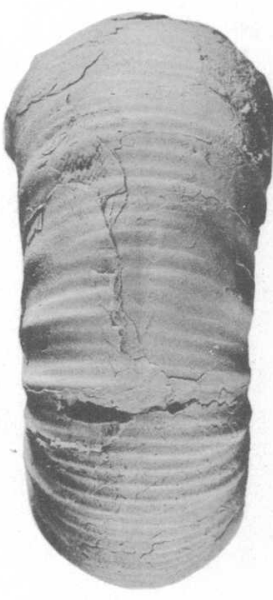

25

SCAPHITES OF THE COLORADO GROUP 


\section{PLATE 11}

[All figures natural size except as indicated on plate]

Figures 1-14. Scaphites impendicostatus Cobban, n. sp. 1-8, Side, front, rear, top, and bottom views, last suture, cross section of shell on straight part of living chamber, and cross section of shell at oral end of living chamber of holotype, an internal mold retaining part of the shell, U.S.N.M. 106686. From a bed of calcareous concretions in the Colorado shale 183-197 feet above top of calcareous member of Greenhorn age at map locality 47. 9, 10, Front and side views of an immature specimen with most of the shell, U.S.N.M. 106687, from the same locality.. 11-13, Bottom, rear, and side views of a coarsely ribbed specimen, an internal mold with part of the shell, U.S.N.M. 106689. From the Colorado shale 500-580 feet below the top at map locality 20. 14, Cross section of septate whorls at diameter of $26 \mathrm{~mm}$. of an incomplete specimen, U.S.N.M. 106688. From a limestone concretion at base of Niobrara shale member of Cody shale at map locality 91 (p. 28). aphites impendicostatus var. erucoides Cobban, n. var. 15, 16, Side and rear views of an immature specimen, an internal mold with nearly complete living chamber, U.S.N.M. 106691, from same locality as figure 14. 17-22, Third from last suture, and bottom, top, front, rear, and side views of holotype, an internal mold, U.S.N.M. 106690. From a sandstone bed at top of Frontier formation at map locality 199. 23, 24, Side and bottom views of an internal mold with some of the shell, U.S.N.M. 106692a. From a bed of calcareous concretions 514-525 feet below top of Colorado shale at map locality 35. 25-28, Side, front, and bottom views, and next to last suture (composite) of a small adult specimen, U.S.N.M. 106692b, possessing fewer ribs than the typical form. From same locality as figures 23,24 (p. 29). 

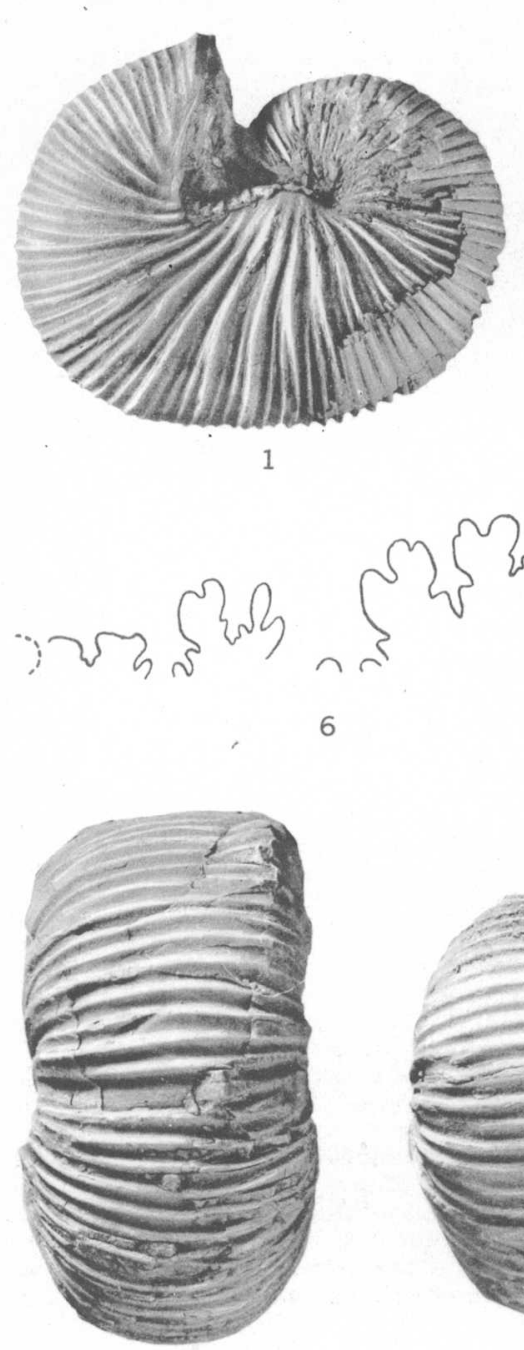

11
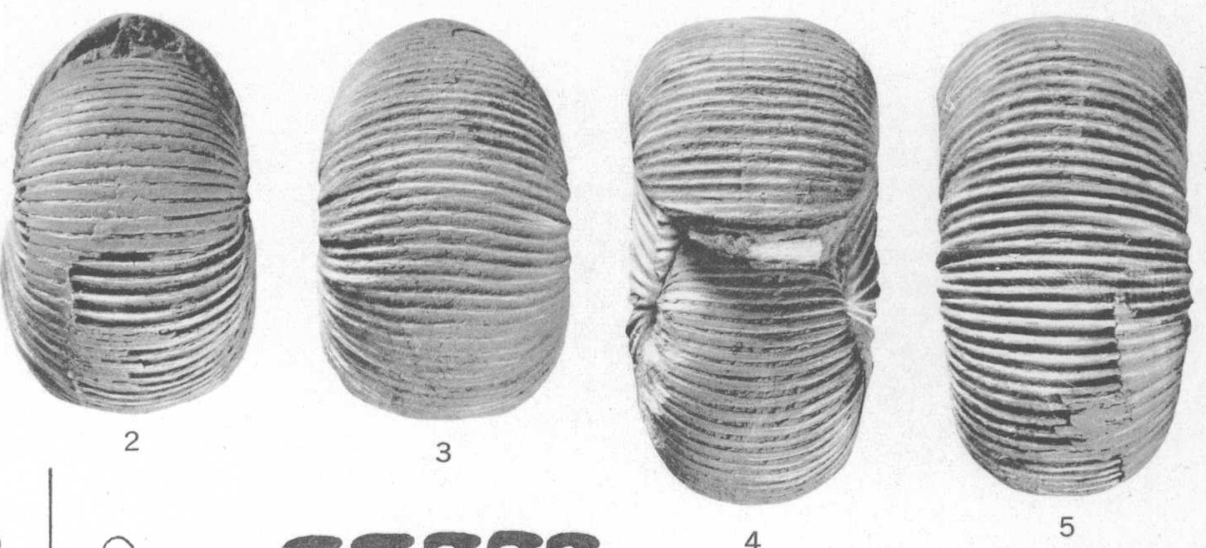

rorror

4
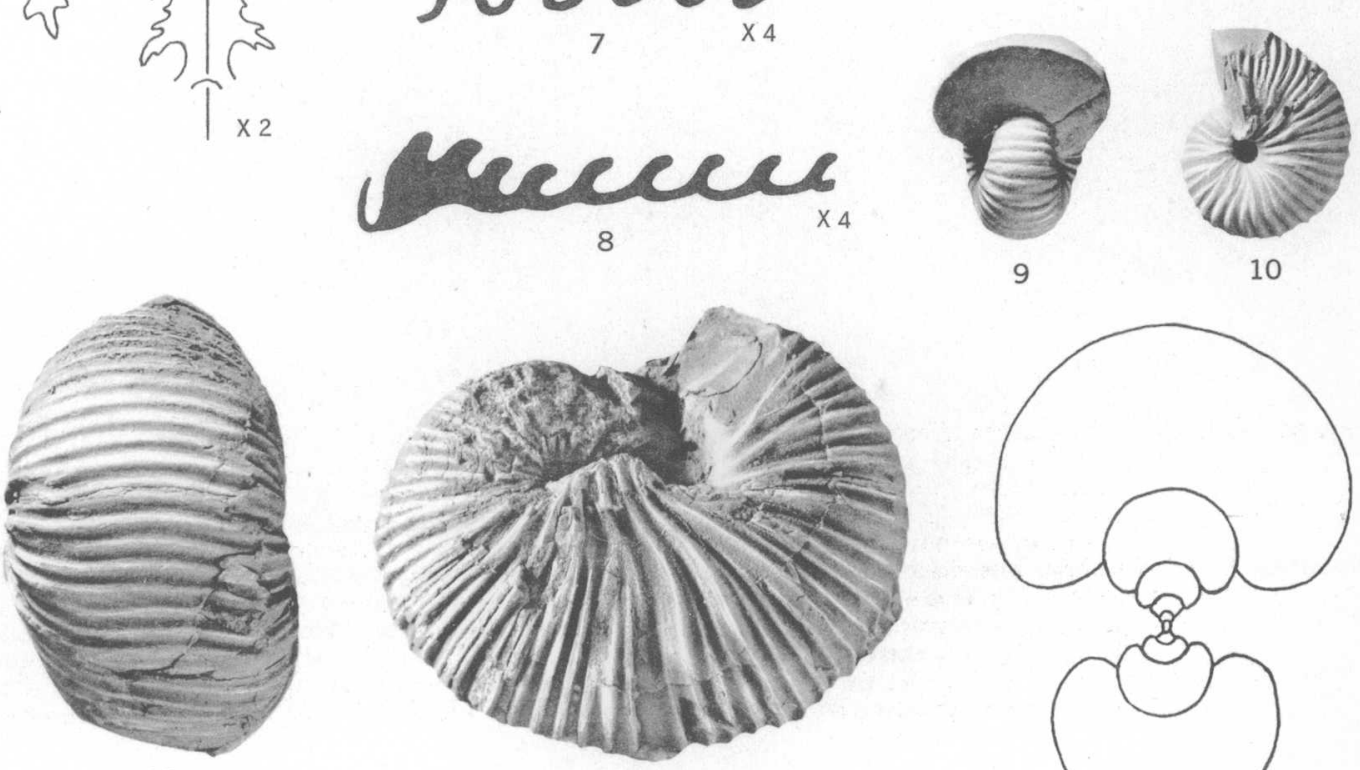

12
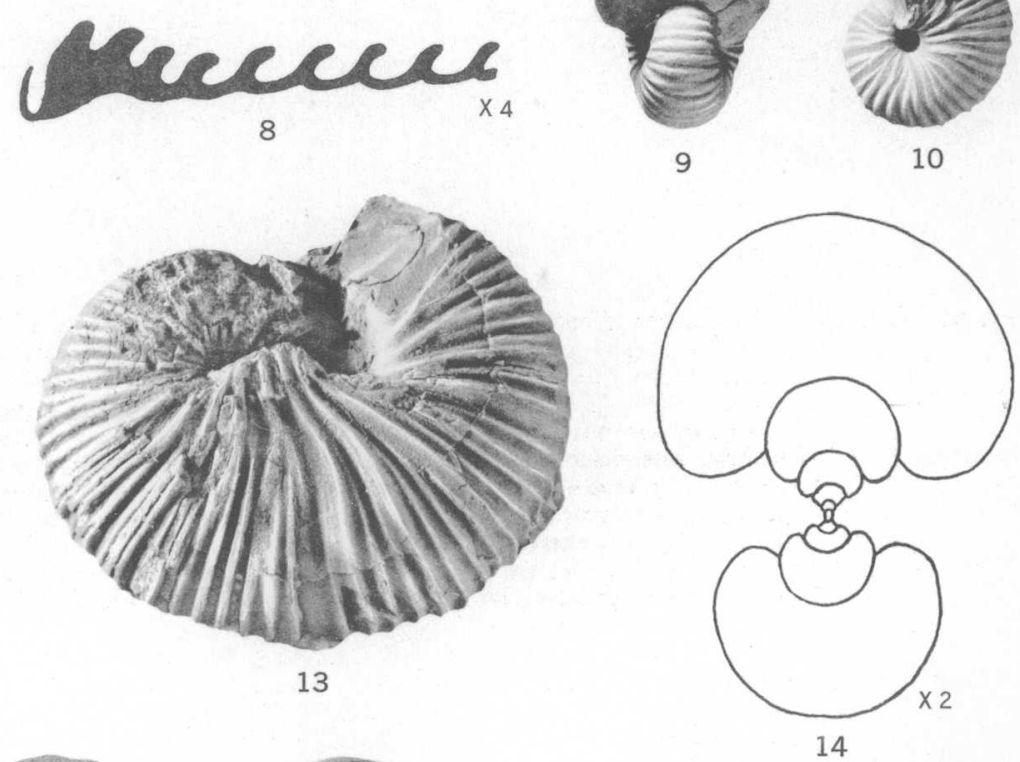

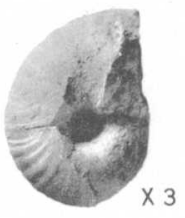

15

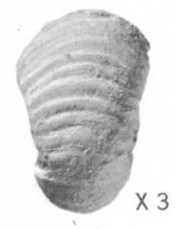

16

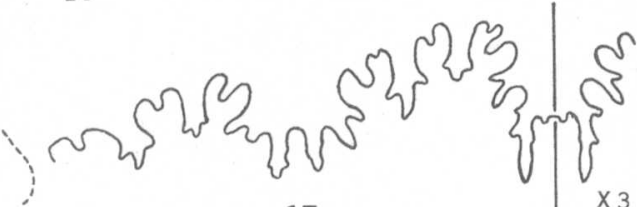

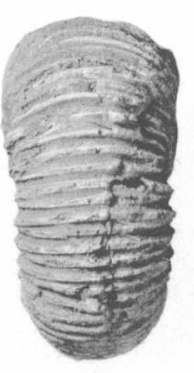

18

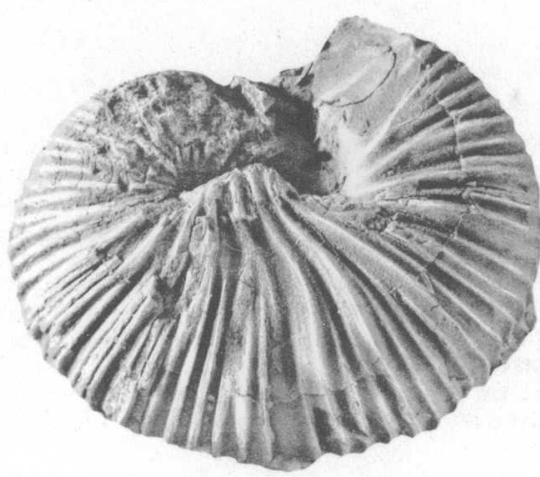

13

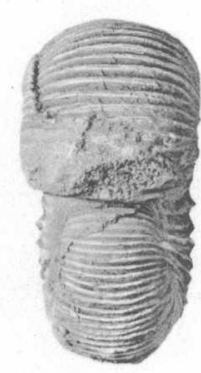

19

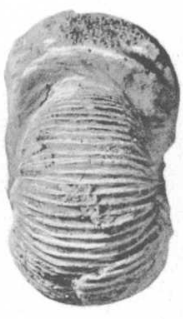

20

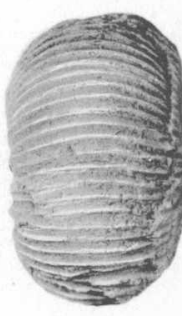

21

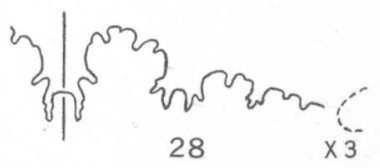

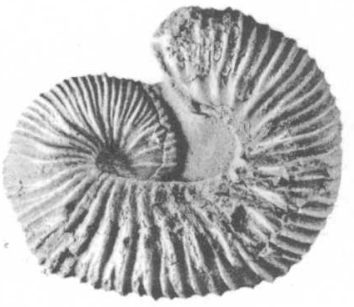

22

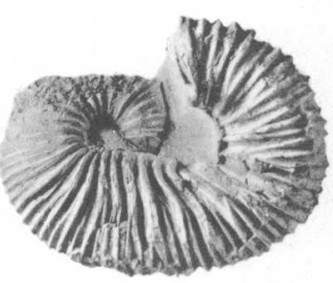

23

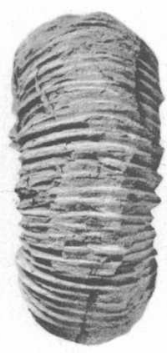

24

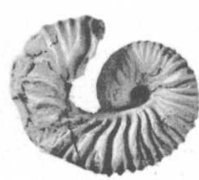

25

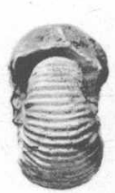

26

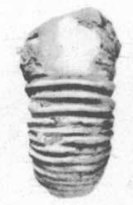

27

SCAPHITES OF THE COLORADO GROUP 


\section{PLATE 12}

[All figures natural size except as indicated on plate]

FIGUREs 1-10. Scaphites ventricosus Meek and Hayden. 1-4, Bottom, top, rear, and side views of a specimen retaining part of the shell, U.S.N.M. 106700. From a sandstone bed 538 feet above.base of Cody shale at map locality 177. 5, 6, Rear and side views of an internal mold of an incomplete adult living chamber, U:S.N.M. 106699, showing the right-angle bend between the aperture and the umbilical wall. From a ferruginous concretion bed 306-392 feet below top of Colorado shale at map locality 18. 7, Suture of a specimen; U.S.N.M. 106698, from same locality as figures 5, 6. 8-10, Side, bottom, and top views of holotype, U.S.N.M. 1903. From Colorado shale at map locality 55 (p. 31). 


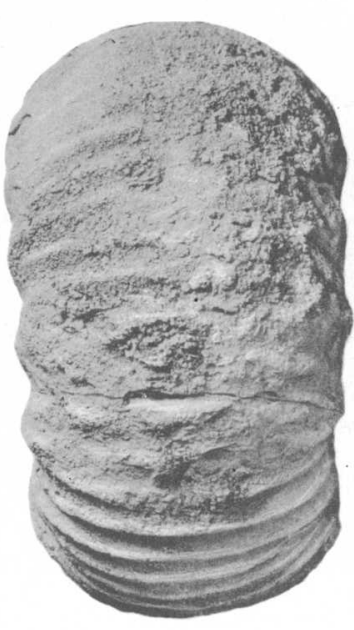

1

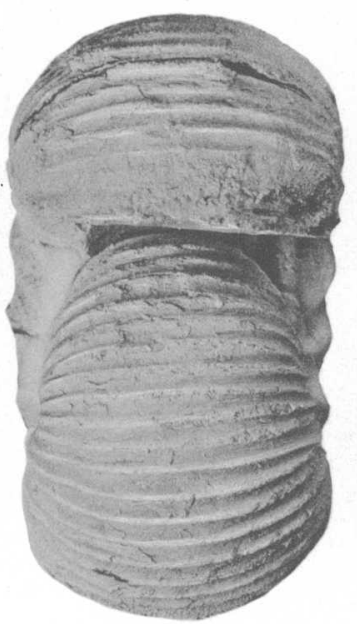

2

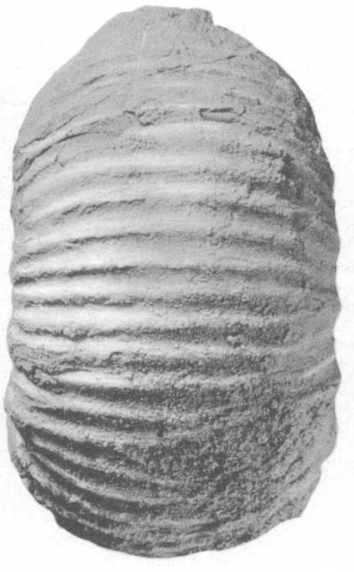

3

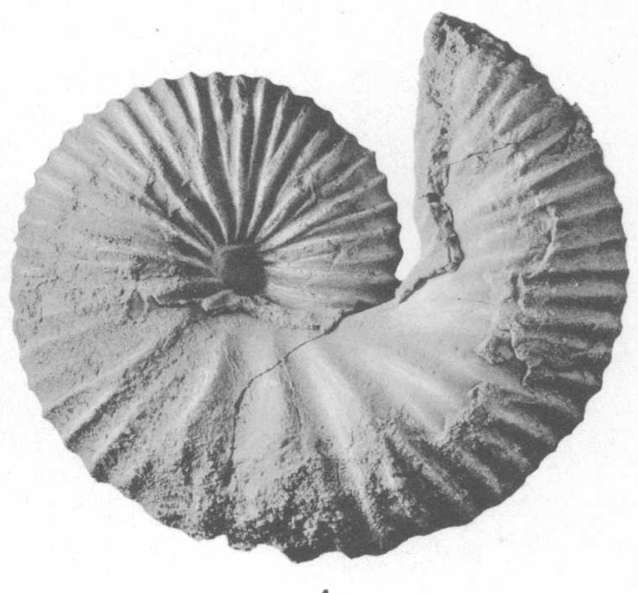

4

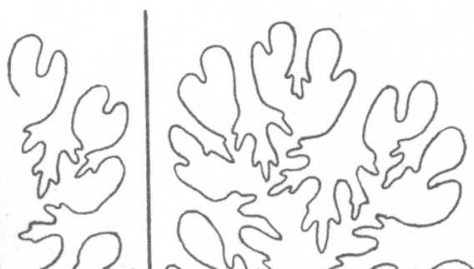

भुज़ी
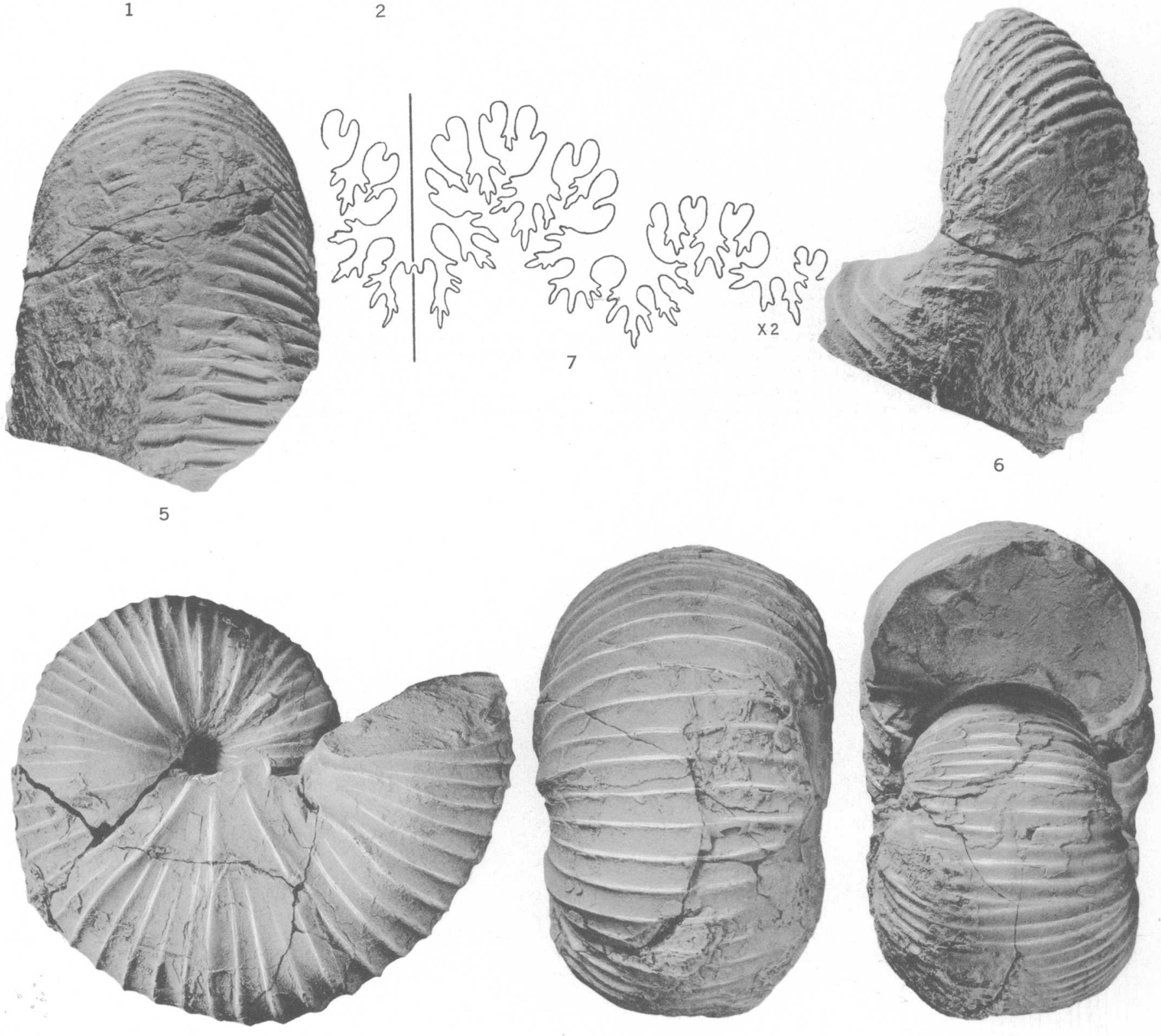

8

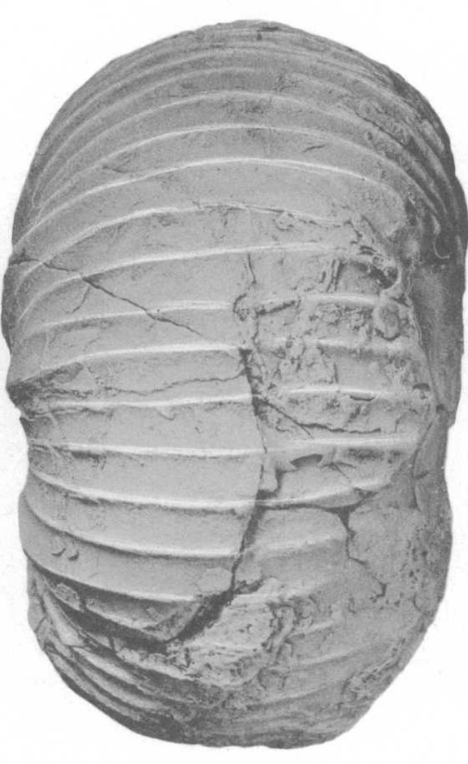

9

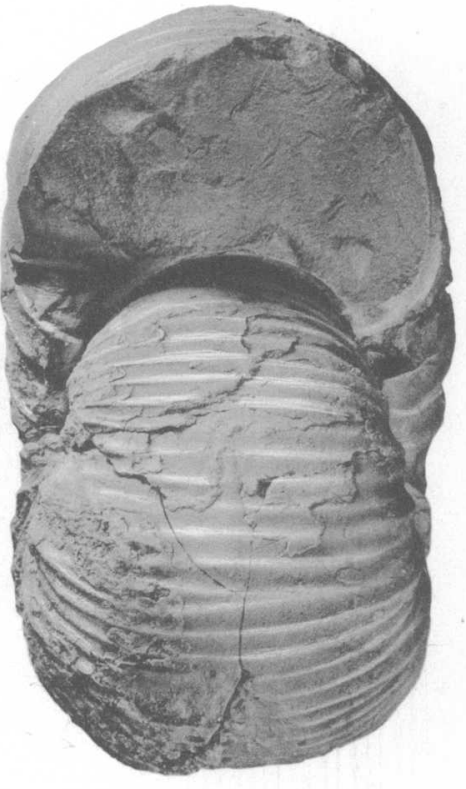

10

SCAPHITES OF THE COLORADO GROUP 
PLATE 13

[A.ll figures natural size except as indicated on plate]

Figures 1-10. Clioscaphites saxitonianus (McLearn). 1-5, Side and rear views and suture of the internal whorls of a specimen, U.S.N.M. 106739b. From a calcareous concretion in the Colorado shale 165 feet below the top at map locality 38 . 6-10, Side, top, rear, and bottom views, and last suture of an internal mold retaining a little of the shell, U.S.N.M. $106739 \mathrm{a}$, from the same löcality (p. 36).

-11-13. Scaphites ventricosus Meek and Hayden. Side, rear, and bottom views of a slightly crushed internal mold, U.S.N.M. 106757. From a ferruginous concretion 330 feet below top of Colorado shale at map locality 39 (p. 31). 


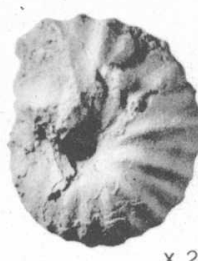

i
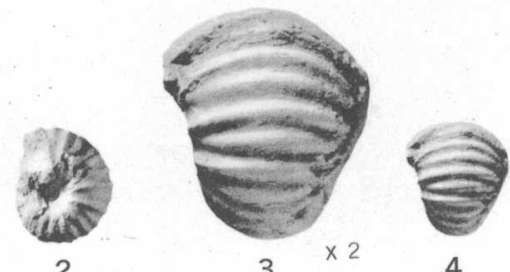

3

4

.

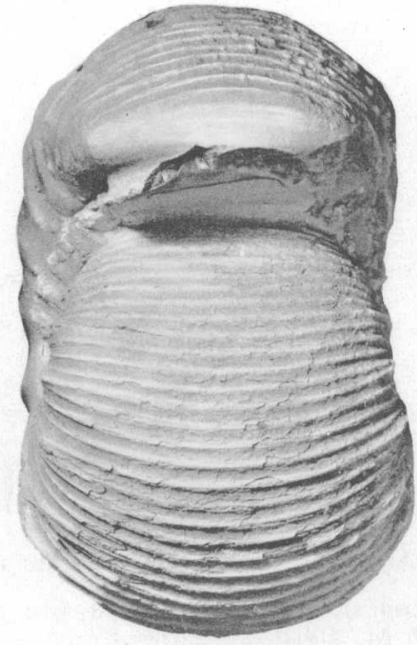

7

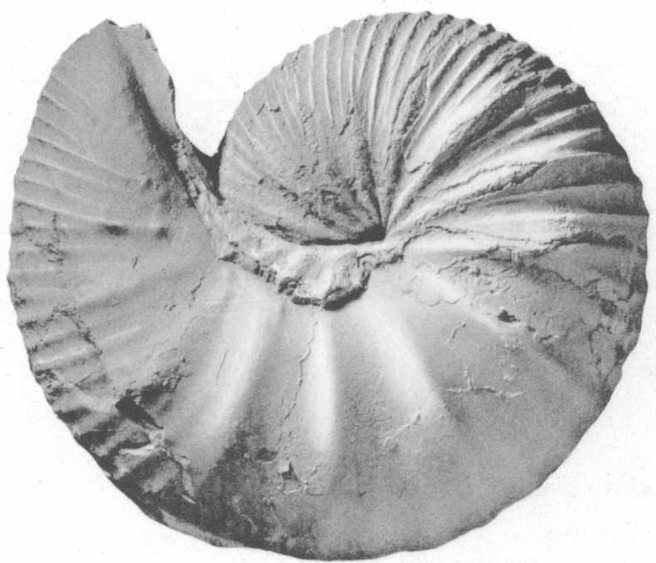

6

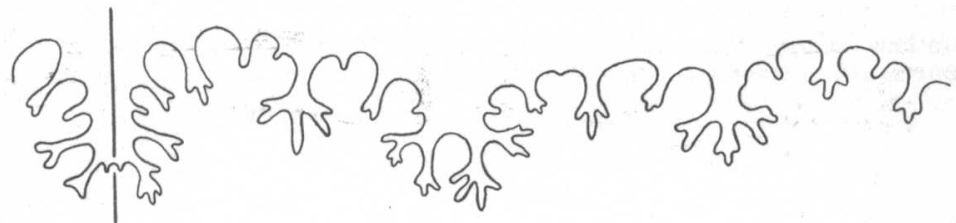

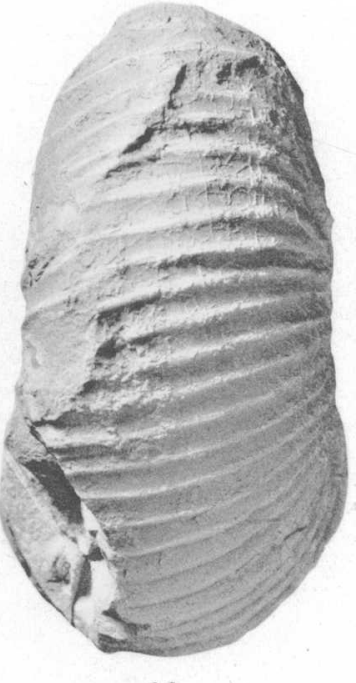

12

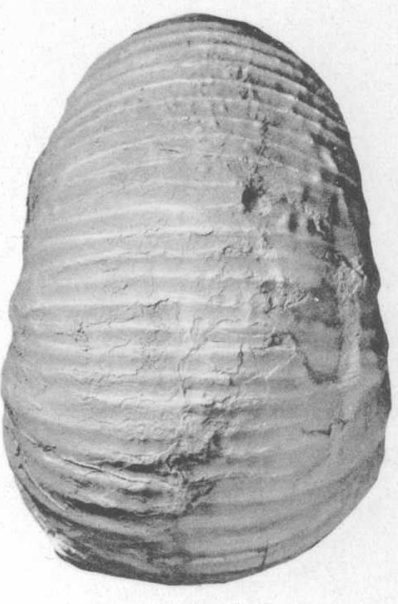

8

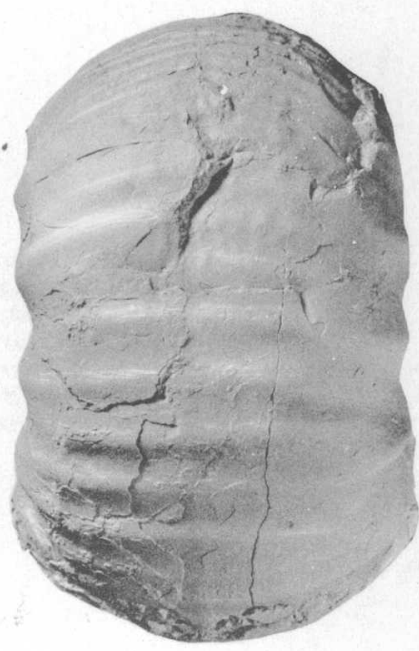

9

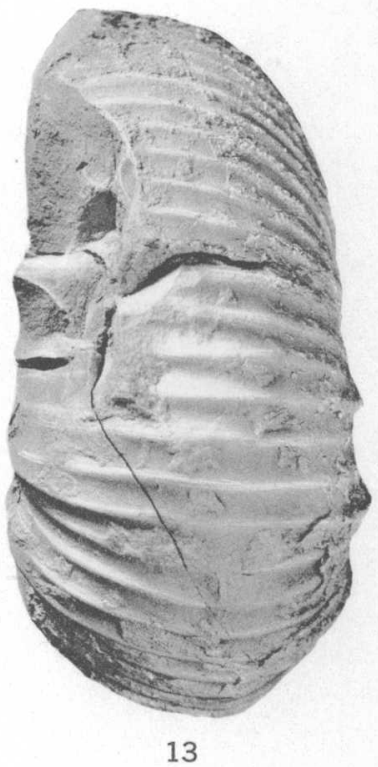

SCAPHITES OF THE COLORADO GROUP 


\section{PLATE 14}

[All figures natural size except as indicated on plate]

Figures 1-10. Scaphites tetonensis Cobban, n. sp. 1-5, Side, rear, top, and bottom views, and next to last suture of a partly crushed small paratype, U.S.N.M. 106708. From Cody shale 1,283 feet above base at map locality 179. 6-10, Side, rear, bottom, and top views, and last suture of holotype, an internal mold, U.S.N.M. 106707. From Cody shale 500 feet above base at map locality 177 (p. 31).

11-16. Scaphites binneyi Reeside. 11-15, Last suture, and top, bottom, rear, and side views of a stout variant, an internal mold, U.S.N.M. 106705. From the Cody shale 574-774 feet above base at map locality 201. 16, Suture at diameter of $22 \mathrm{~mm}$. of a specimen, U.S.N.M. 106706. From the Cody shale 450-574 feet above the base at map locality 200 (p. 32).

17-21. Scaphites interjectus Reeside. Seventh from last suture, and side, rear, top, and bottom views of a small specimen, U.S.N.M. 106701. From a calcareous concretion in the Cody shale about 1,500 feet above the base at map locality 189 (p. 32). 


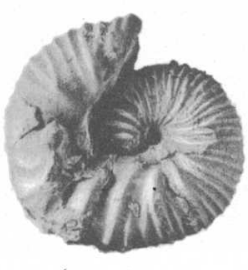

1
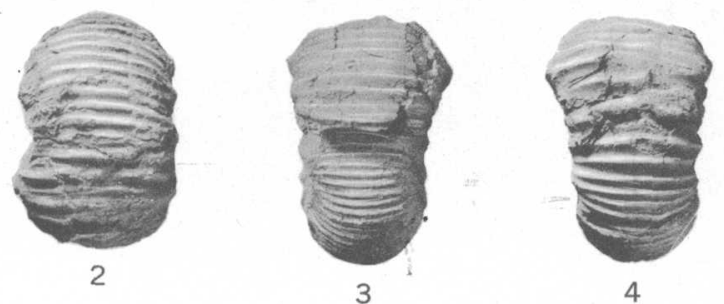

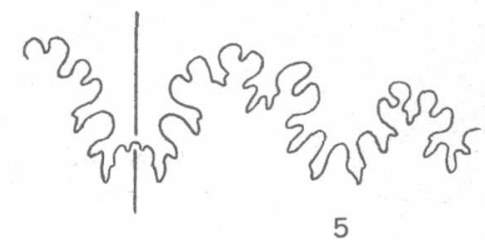

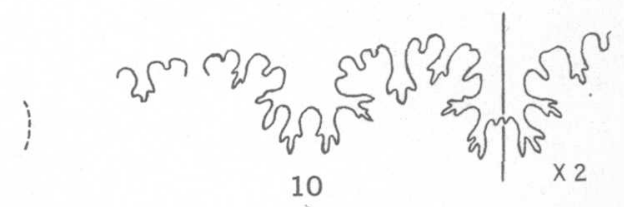

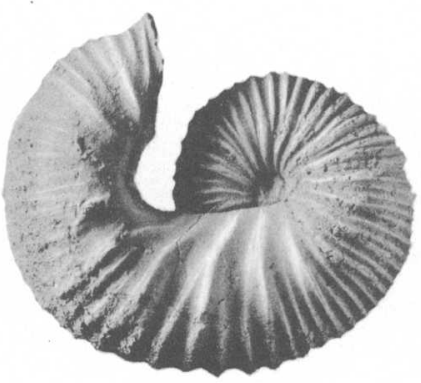

6
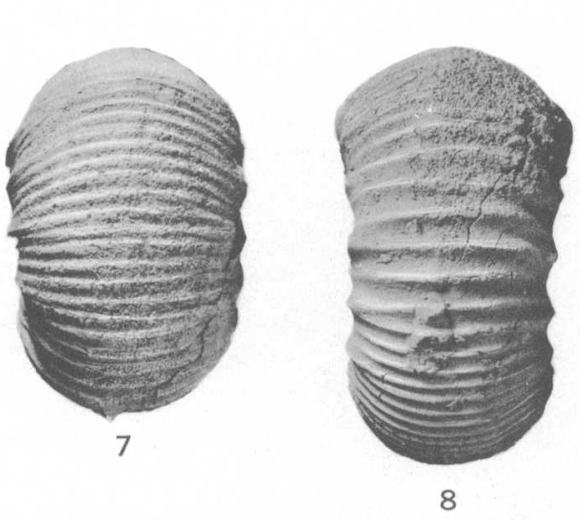

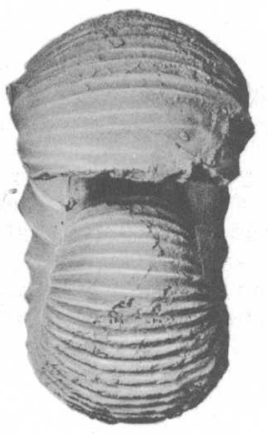

9

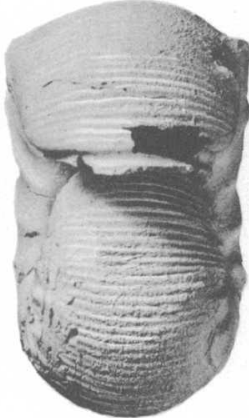

12

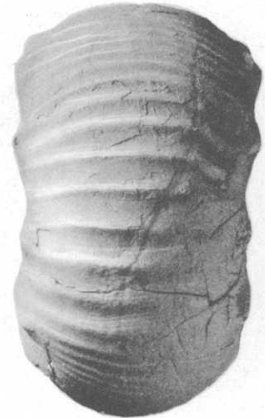

13

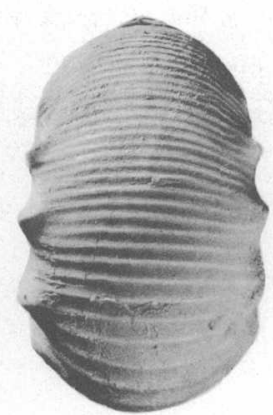

14

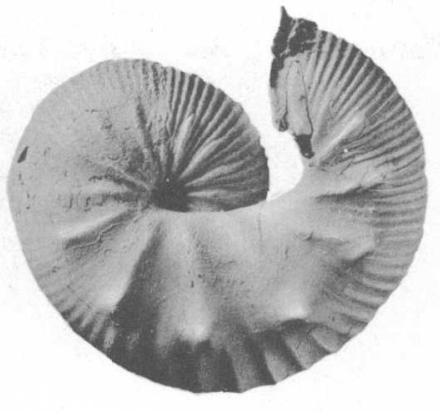

15

तथ ।

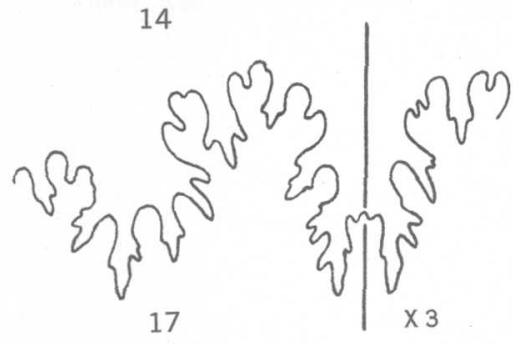

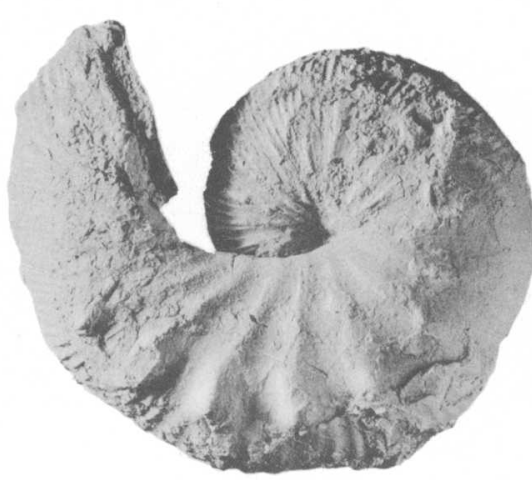

18

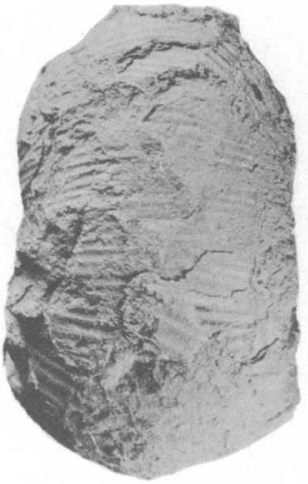

19

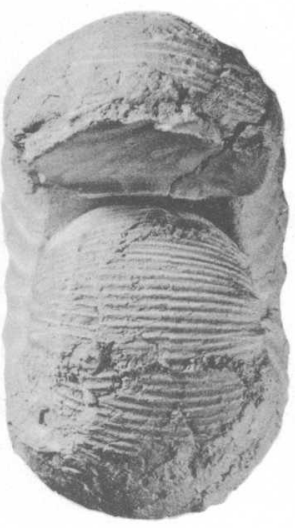

20

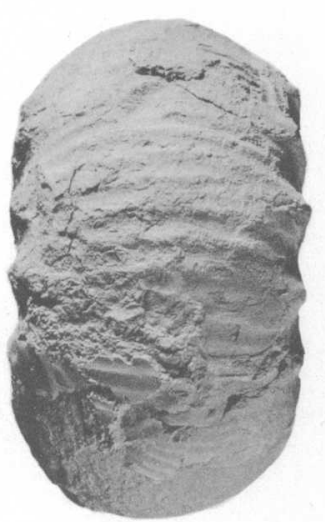

21

SCAPHITES OF THE COLORADO GROUP 


\section{PLATE 15}

[All figures natural size except as indicated on plate]

Figures 1-5. Scaphites depressus Reeside var. stantoni Reeside. Side, rear, top, and bottom views, and last suture of a specimen, U.S.N.M. 106695, transitional from S. ventricosus. From a ferruginous concretion 370 feet above base of Cody shale at map locality 148 (p. 33).

6-8. Scaphites depressus Reeside. Second from last suture, and side and rear views of a specimen retaining much of the shell, U.S.N.M. 106693. From a calcareous concretion about 700 feet above base of Cody shale at map locality 145 (p. 32). 

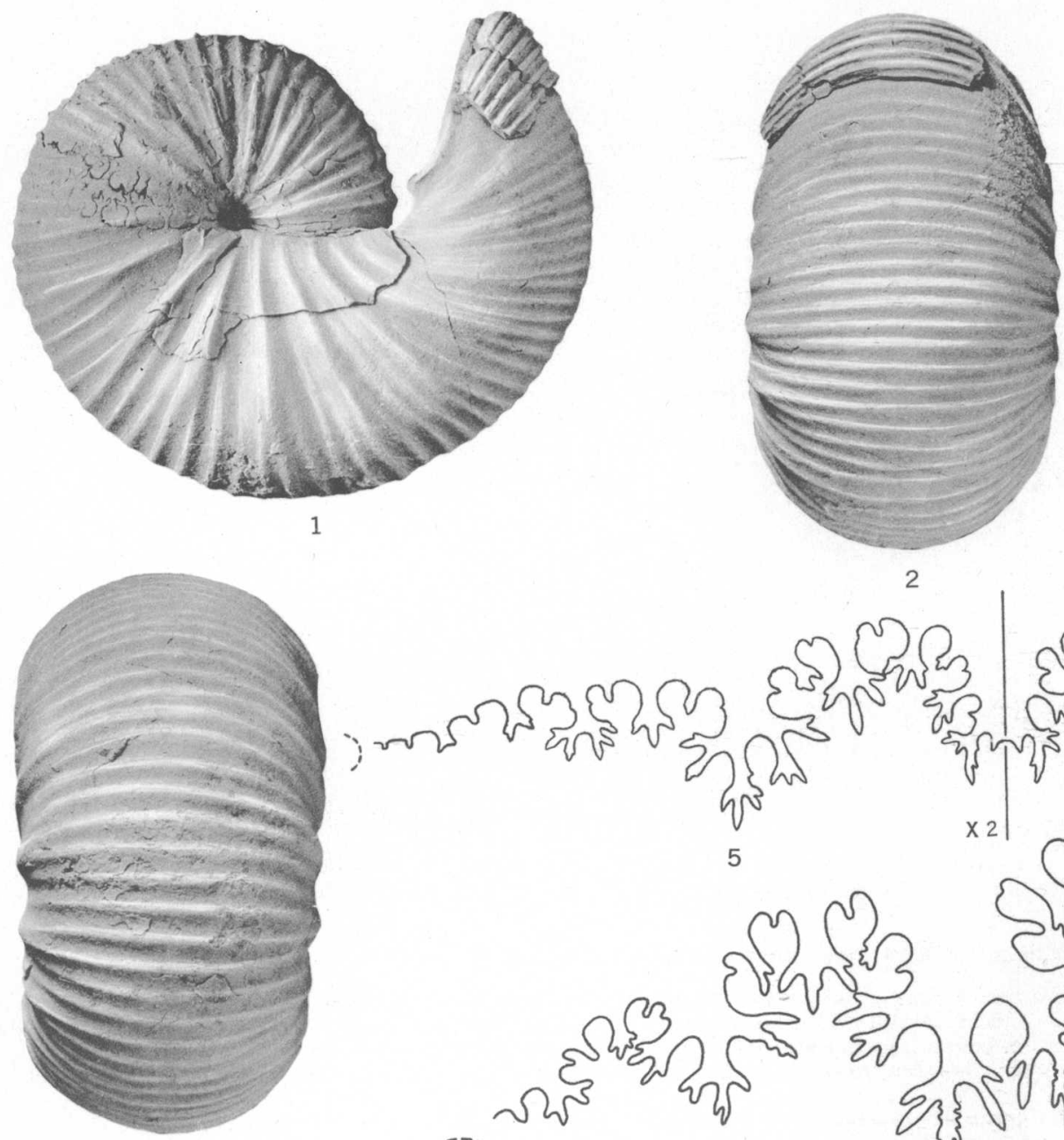

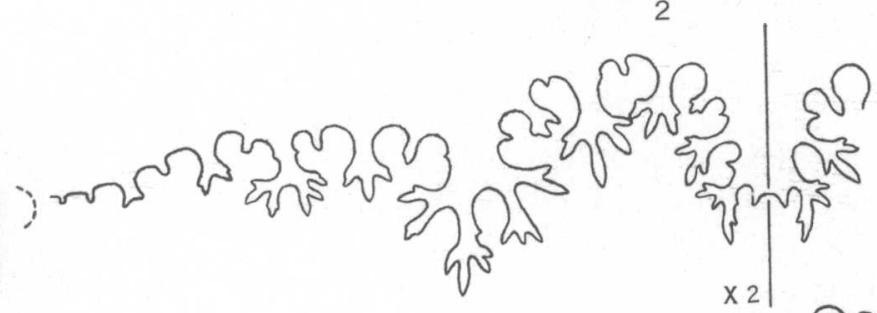

4

5

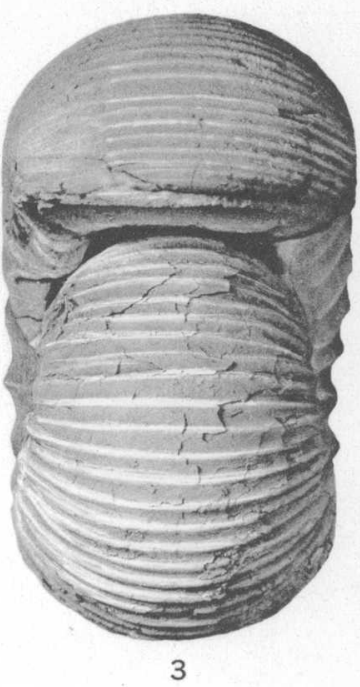

,

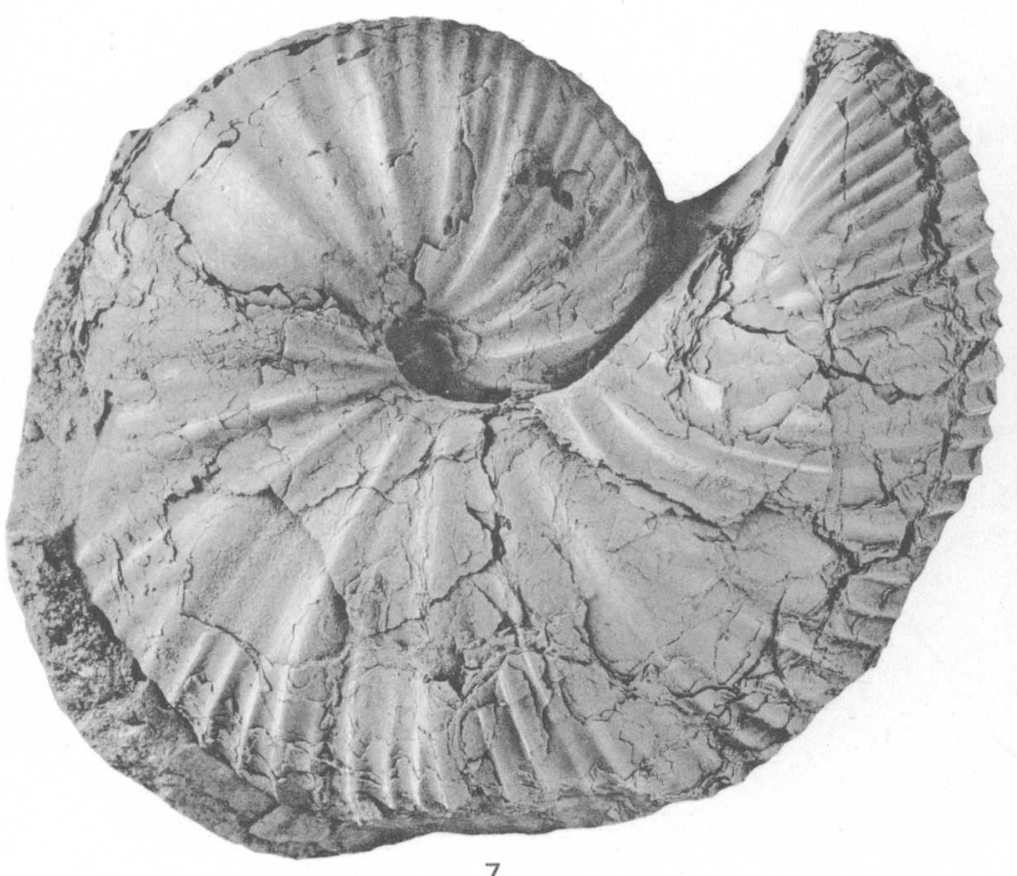
क्ञ

\{ूर 2 ?

6

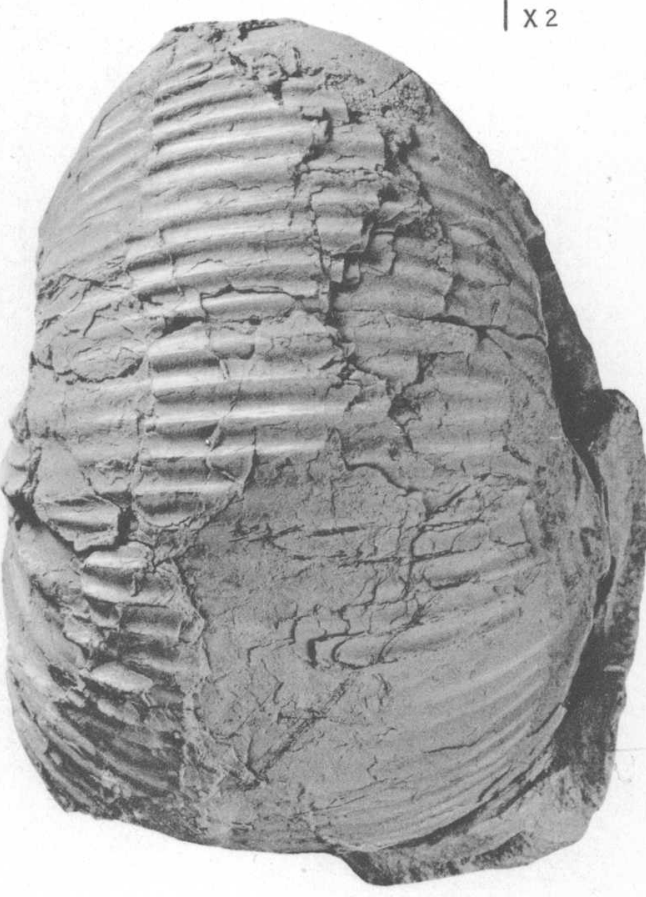

8

SCAPHITES OF THE COLORADO GROUP 


\section{PLATE 16}

[All figures natural size except as indicated on plate]

Figures 1-11. Clioscaphites montanensis Cobban, n. sp. . From a bed of calcareous concretions 234-252 feet below top of Colorado shale at map locality 33. 1-5, Side, rear, top, and bottom views, and last suture of holotype, U.S. N.M. 106716. 6-8. Side, front, and rear views of an internal mold of septate whorls at diameter of $13 \mathrm{~mm}$. of a specimen, U.S.N.M. 106717c. 9-11, Side, top, and bottom views of an internal mold retaining some of the shell, U.S.N.M. 106717b (p. 34).

12-14. Clioscaphites montanensis Cobban var. hesperius Cobban, n. var. Side, rear, and bottom views of holotype, U.S.N.M. 106718 , from the same locality (p. 34). 

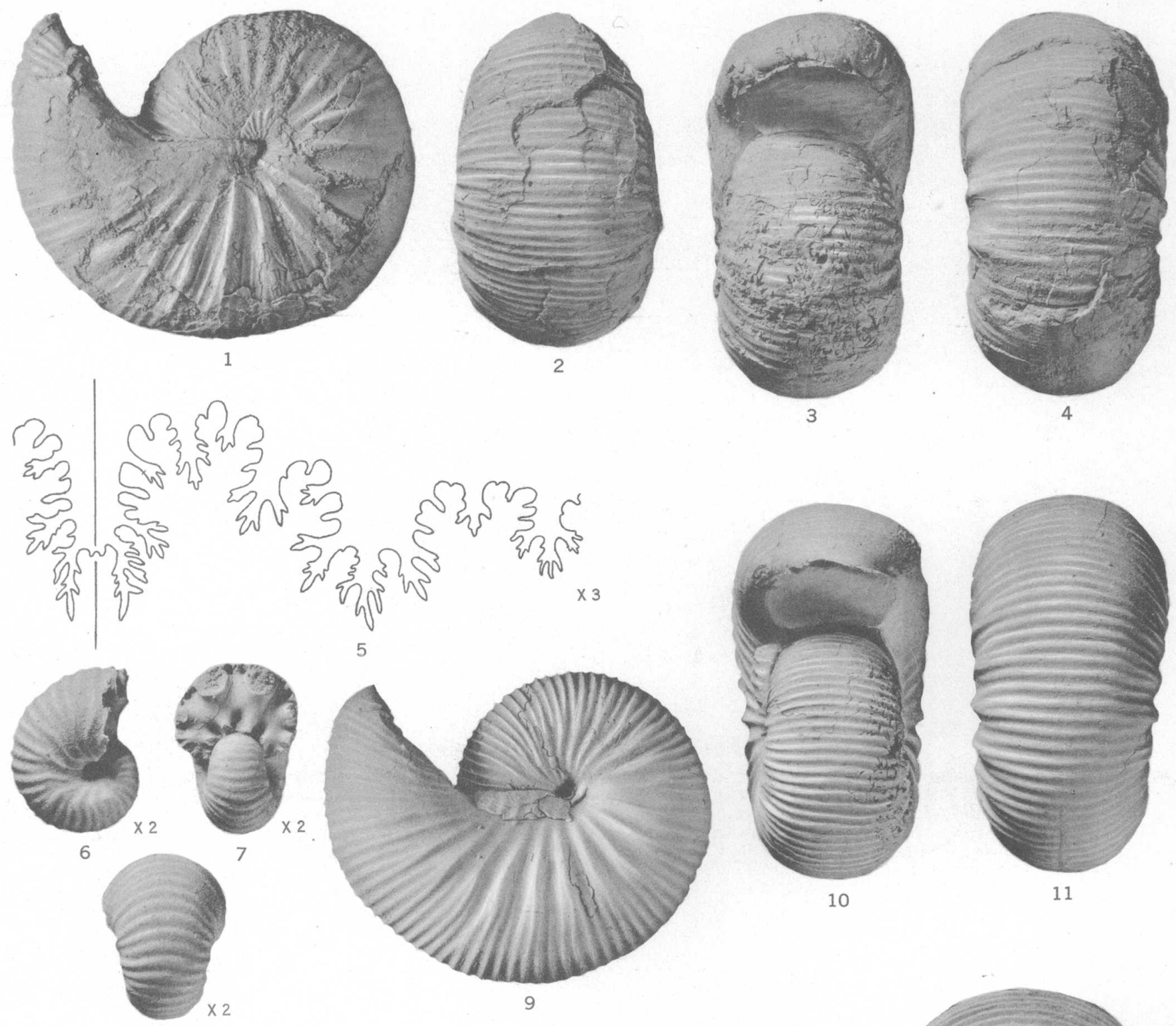

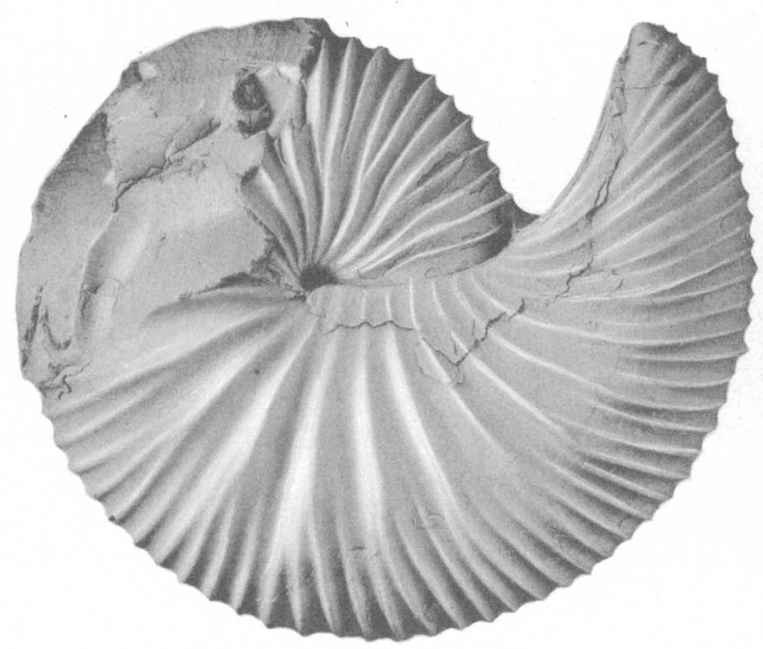

12

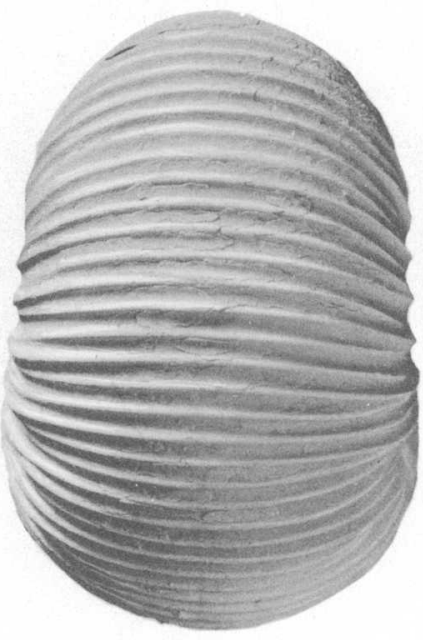

13

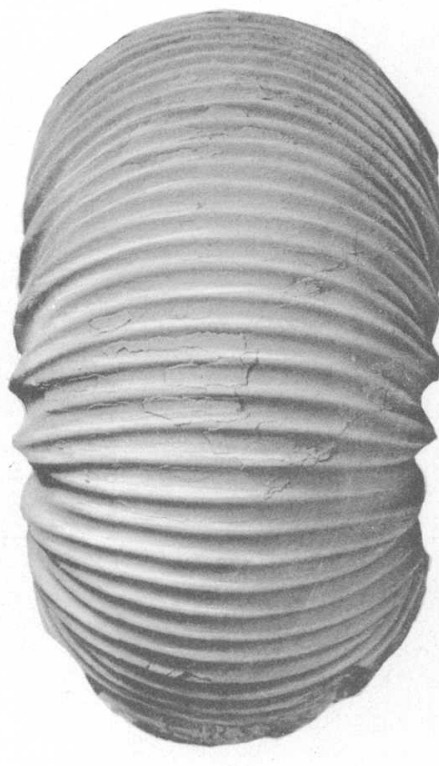

14

SCAPHITES OF THE COLORADO GROUP 
PLATE 17

[All figures natural size except as indicated on plate]

Figures 1-3. Clioscaphites montanensis Cobban, n. sp. Side and bottom views, and suture at diameter of $56 \mathrm{~mm}$. of a large internal mold, U.S.N.M. 106717d. From a bed of calcareous concretions 234-252 feet below top of Colorado shale at map locality 33 (p. 34).

4-7. Clioscaphites montanensis Cobban var. hesperius Cobban, $n$. var. Bottom, front, and side views, and second from last suture of a large internal mold, U.S.N.M. 106719, from the same locality (p. 34). 

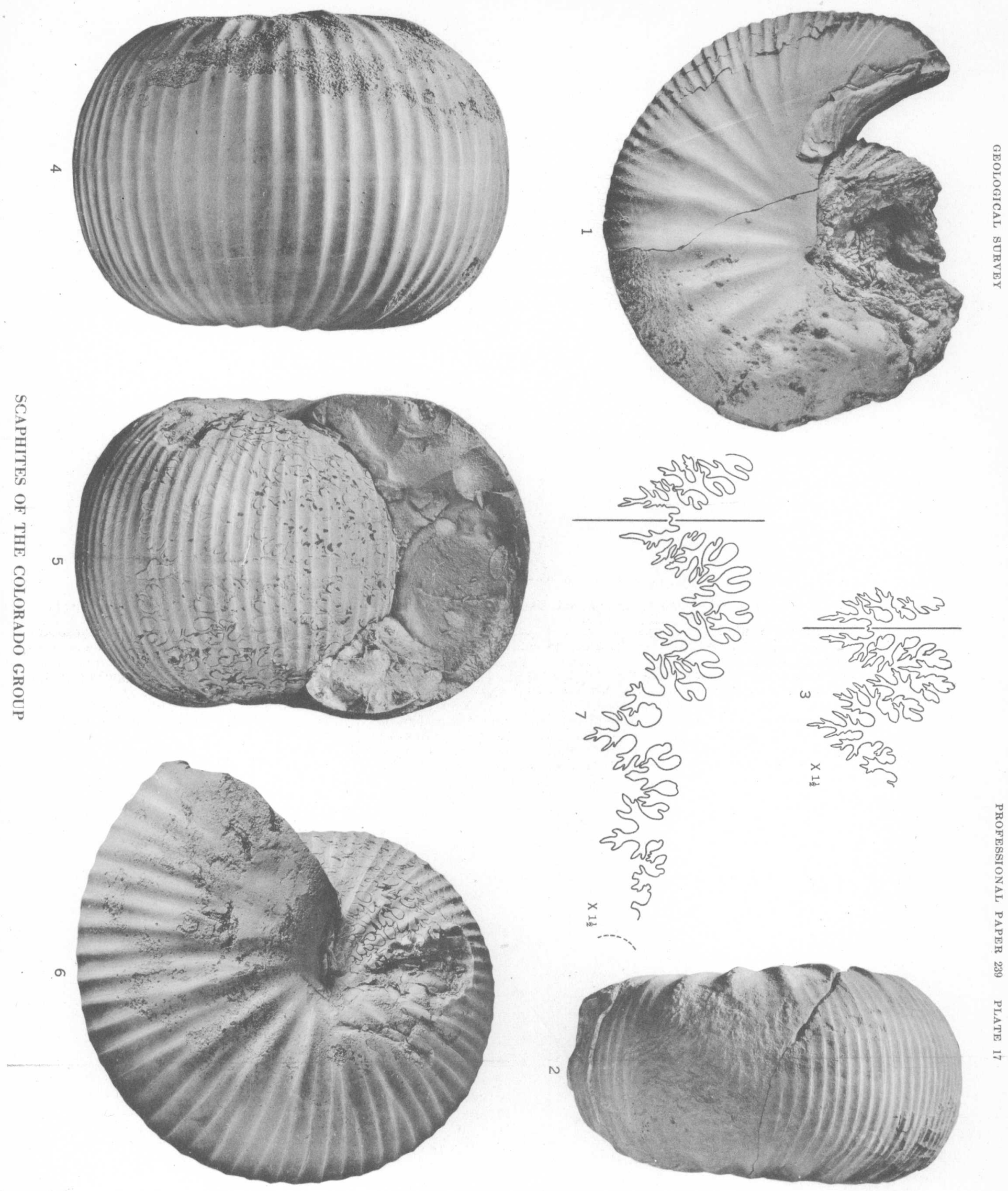
PLATE 18

[All figures natural size except as indicated on plate]

Figures 1-6. Scaphites coloradensis Cobban, n. sp. From calcareous concretions 234-252 feet below top of Colorado shale at map locality 33. 1-4, Bottom, top, and side views, and next to last suture of holotype, an internal mold, U.S.N.M. 106715. 5, 6, Fourth from last suture, and cross section through septate whorls and oral end of living chamber of an adult specimen, U.S.N.M. 106714 (p. 33).

7-27. Clioscaphites vermiformis (Meek and Hayden). From same locality as figures 1-6. 7-11, Side, rear, top, and bottom views, and last suture of a small specimen retaining the shell on the septate whorls and partly on the living chamber U.S.N.M. 106713d. 12-15, Side, bottom, front, and rear views of the internal whorls at diameter of $18.5 \mathrm{~mm}$. of a specimen retaining much of the shell, U.S.N.M. 106713e. 16-19, Side, front, and rear views, and last suture of an immature specimen, an internal mold with complete living chamber, U.S.N.M. 106713f. 20, 21, Side and bottom views of the internal mold of the older part of the living chamber of a sharp-ribbed variant, U.S. N.M. $106713 \mathrm{~b}$. 22,23 , Bottom and side views of the smallest known adult specimen, U.S.N.M. 106713c. 24-27, Side, rear, bottom, and top views of a slightly larger than average adult specimen, U.S.N.M. 106713a (p. 35). 

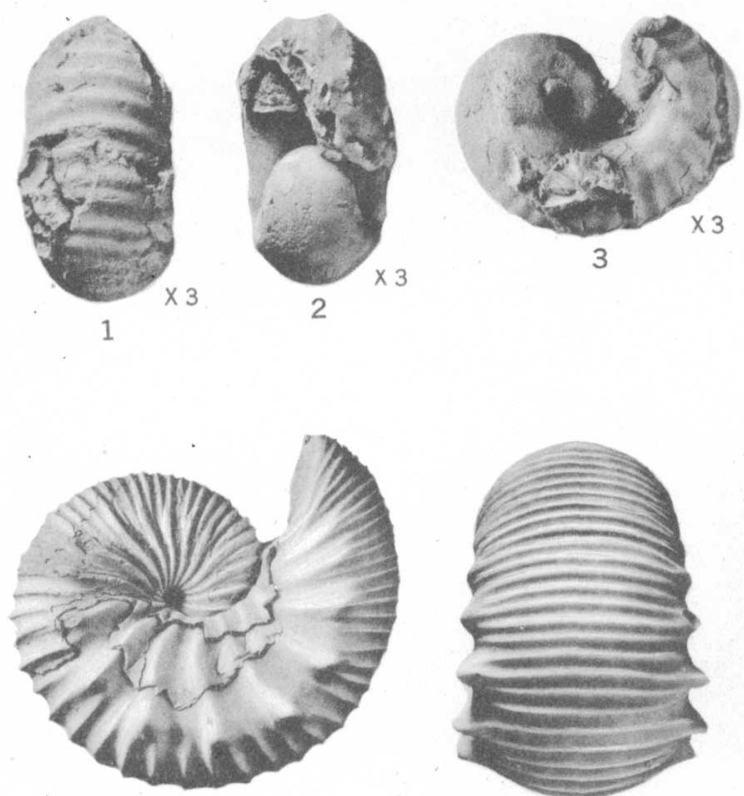

7

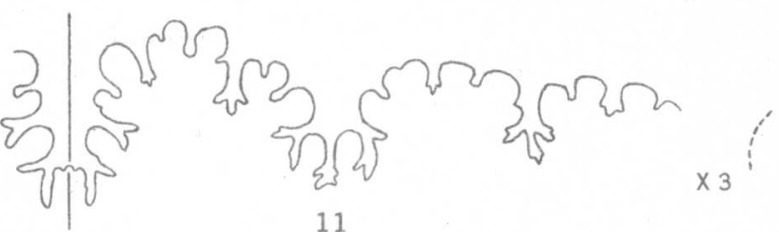
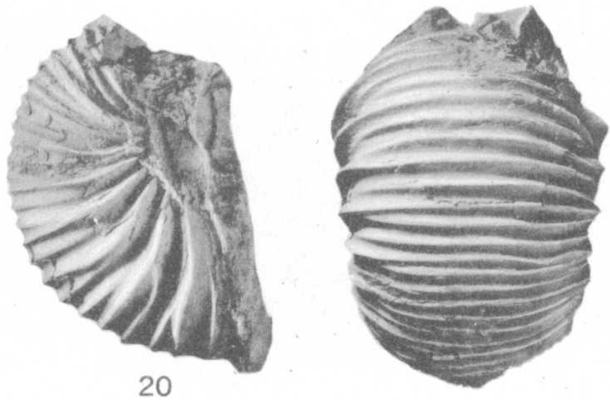

21

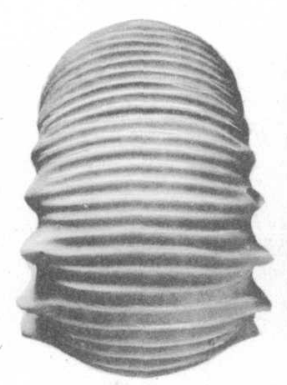

8

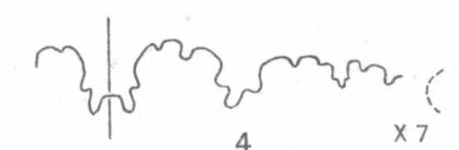

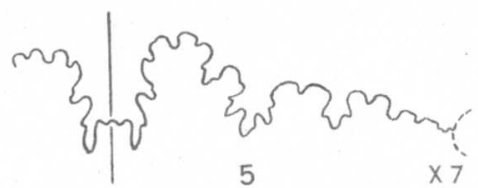

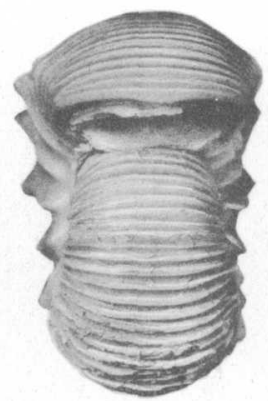

9

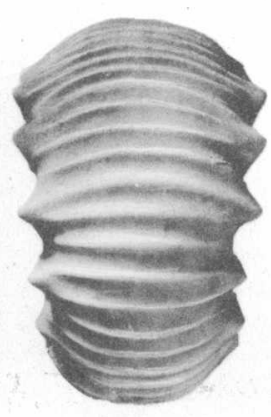

10
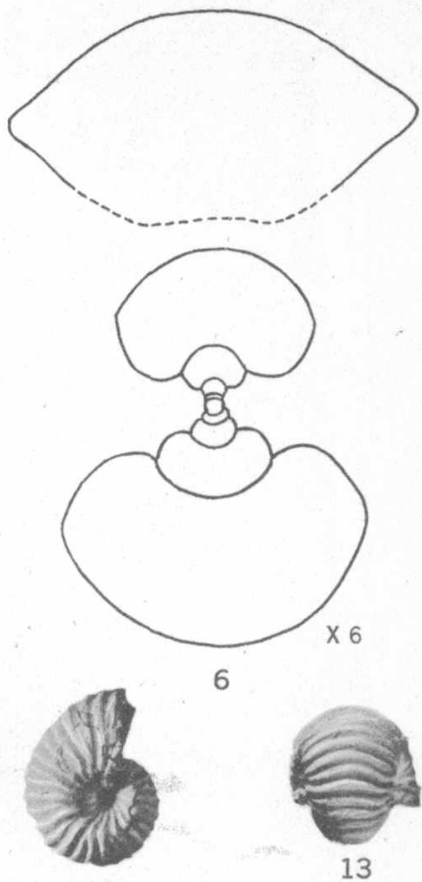

12

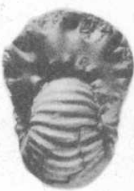

14
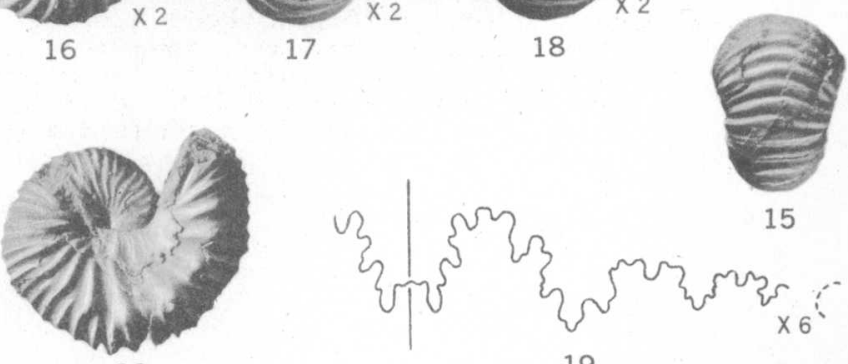

$\xi \int^{2} \xi_{3}$

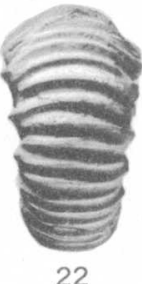

17

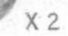

18

15
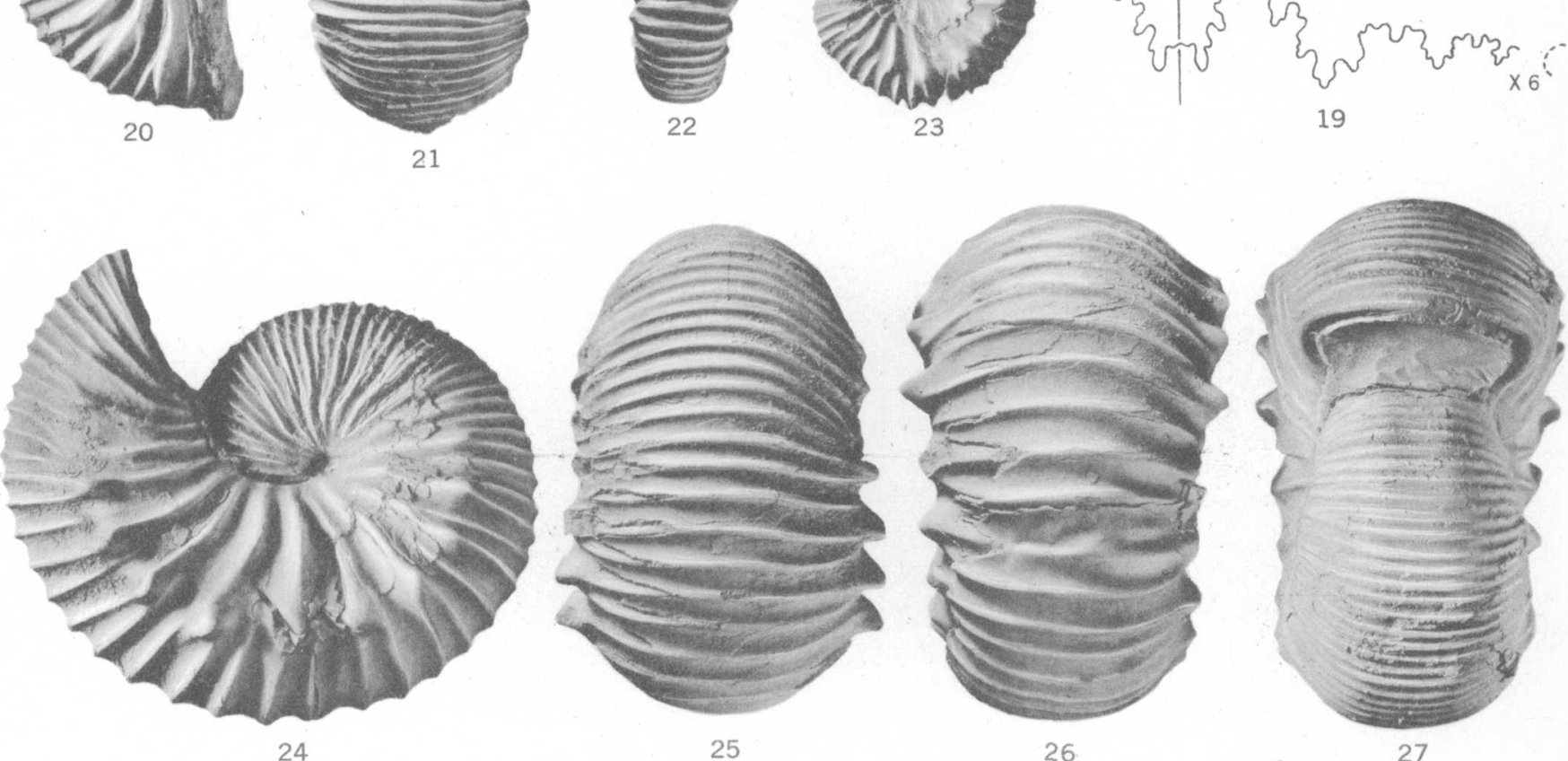

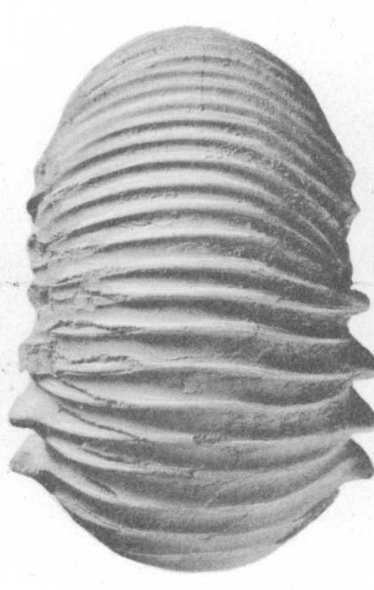

25

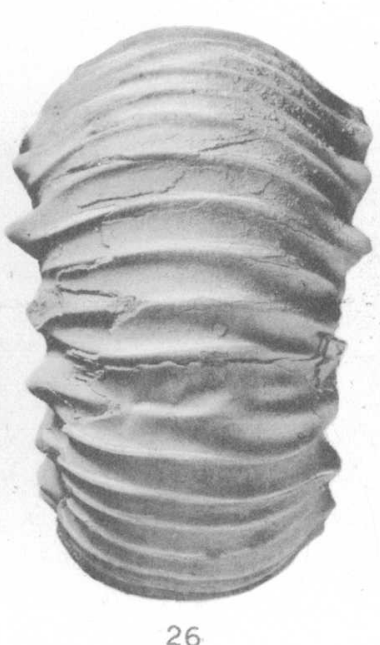

26

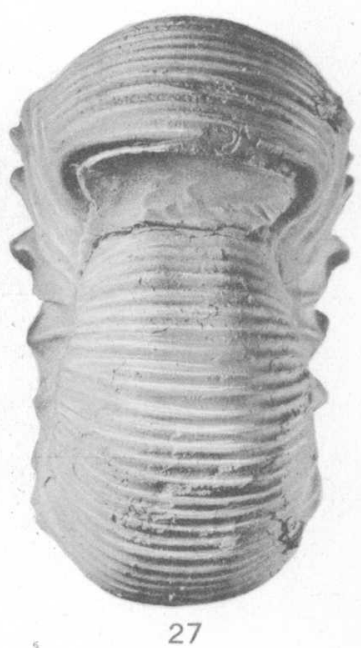

SCAPHITES OF THE COLORADO GROUP 


\section{PLATE 19}

[All figures natural size except as indicated on plate]

Figures 1-10. Clioscaphites vermiformis (Meek and Hayden) var. toolensis Cobban, n. var. 1-5, Side, top, bottom, and rear views, and second suture (composite) from last of holotype, U.S.N.M. 106709 . From a bed of calcareous concretions 234-252 feet below top of Colorado shale at map locality 33. 6-8, Last suture, and side and top views of the septate coil and part of the living chamber of a densely ribbed specimen, U.S.N.M. 106712. From a calcareous concretion near middle of Cody shale at map locality 98. 9, Fragment of the living chamber of the largest known specimen, U.S.N.M. 106710, from same locality as figures 1-5. 10, Cross section of septate whorls of an adult, U.S.N.M. 106711. From a calcareous concretion in the Niobrara member of the Cody shale at map locality 96 (p. 36). 

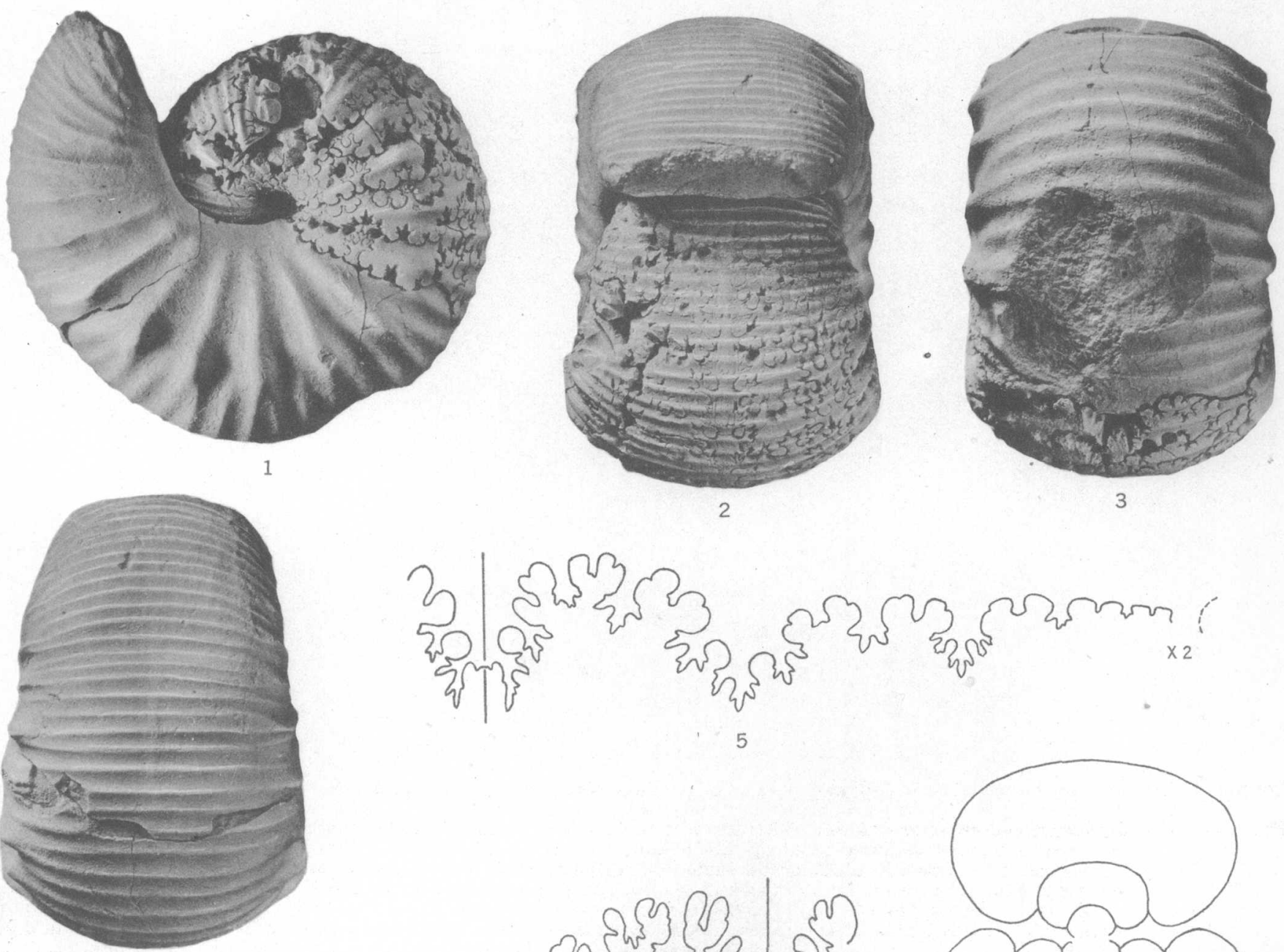

$$
\text { 3.1. }
$$

4

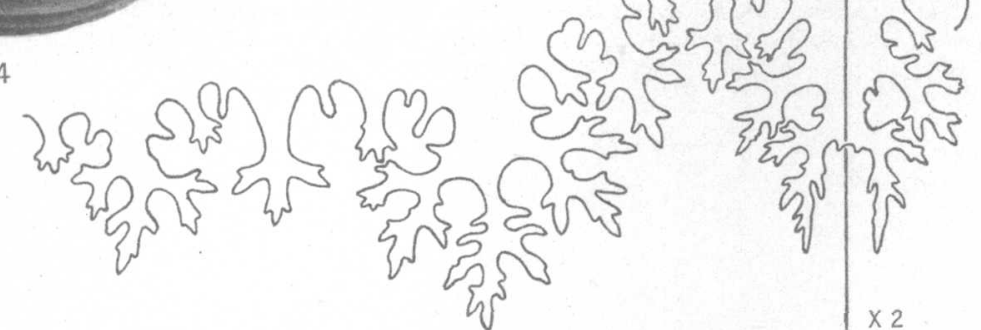

6

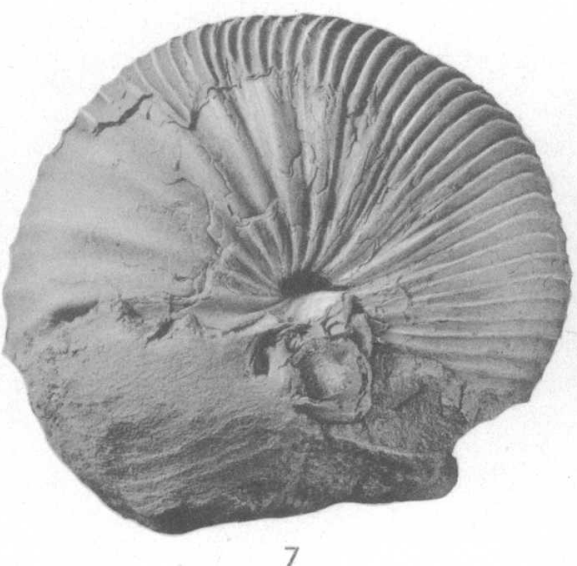

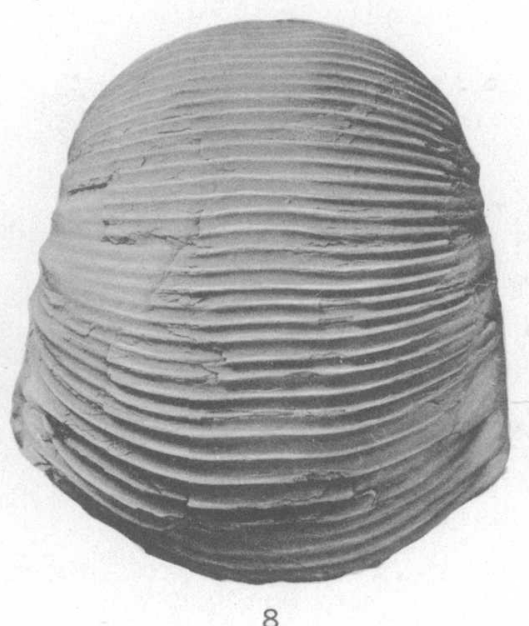

8

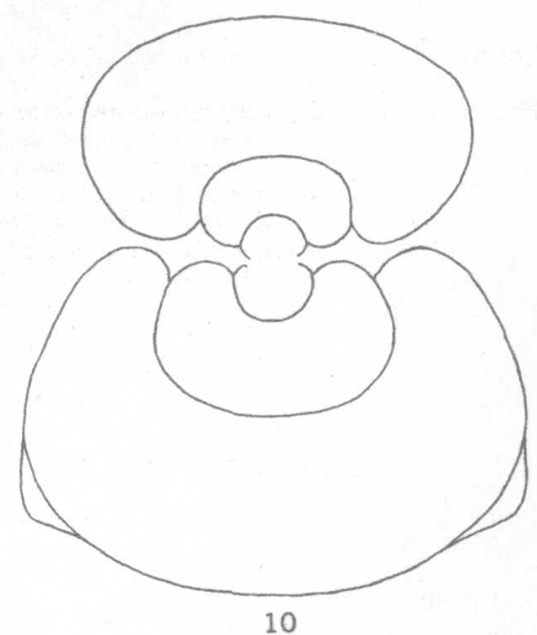

SCAPHITES OF THE COLORADO GROUP

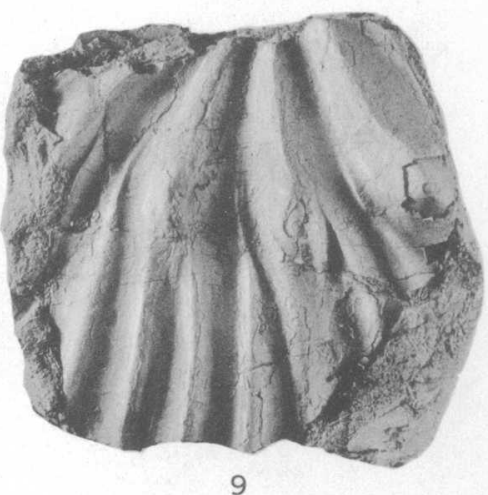


PLATE 20

[All figures natural size except as indicated on plate]

Frgures 1-4. Clioscaphites montanensis Cobban, n. sp. From calcareous concretions 234-252 feet below top of Colorado shale at map locality 33. 1, Cross section of septate whorls of an internal mold at diameter of $38 \mathrm{~mm}$., U.S.N.M. $106726 \mathrm{~b}$. 2-4, Side and front views, and last suture of the internal mold of an immature specimen with complete living chamber, U.S.N.M. $106726 a$ (p. 34).

5-7. Clioscaphites saxitonianus (McLearn) var. keytei Cobban, n. var. Side, top, and bottom views of holotype, an internal mold retaining part of the shell, U.S.N.M. 106727. From a calcareous concretion near top of Apishapa shale at map locality 266 (p. 37).

8-11. Clioscaphites? choteauensis Cobban, n. sp. Side, bottom, and rear views, and last suture of holotype, U.S.N.M. 106728. From a calcareous concretion near top of Colorado shale at map locality 44 (p. 38)

12-16. Clioscaphites platygastrus Cobban, n. sp. Last suture, and side, rear, top, and bottom views of holotype, an internal mold, U.S.N.M. 106729. From a calcareous concretion in upper part of Colorado shale at map locality 10 (p. 36).

, 

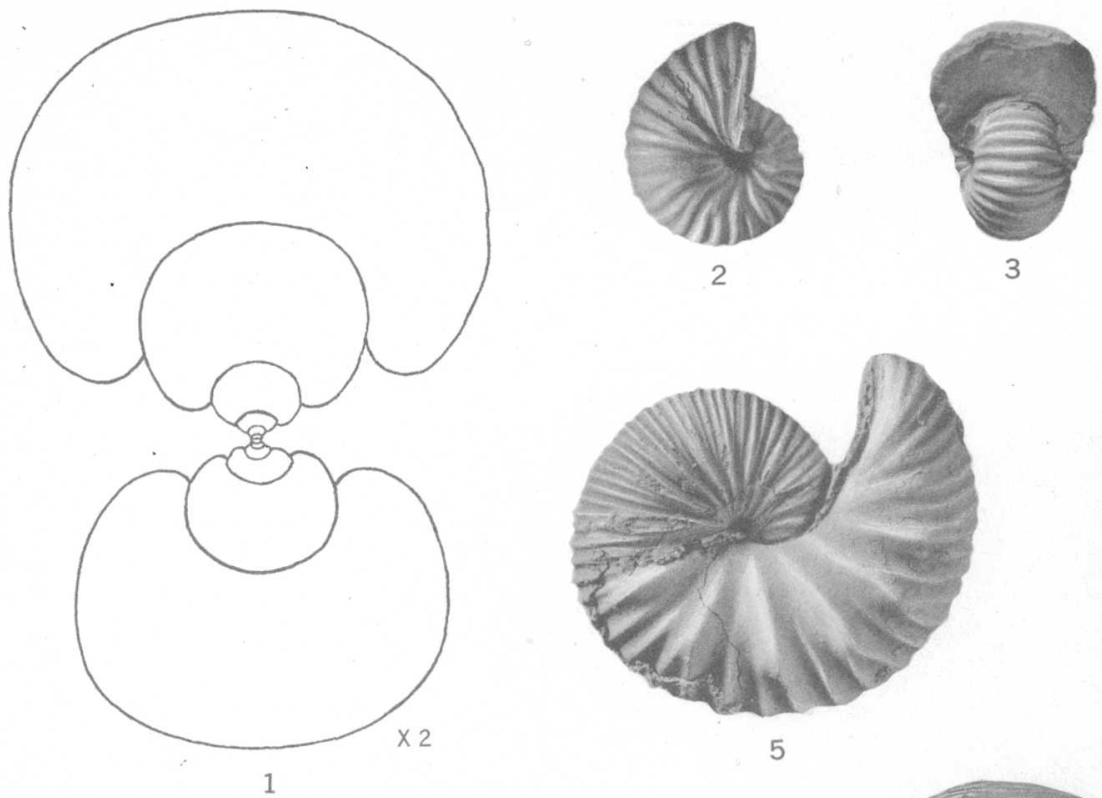

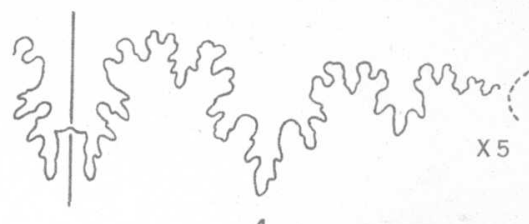
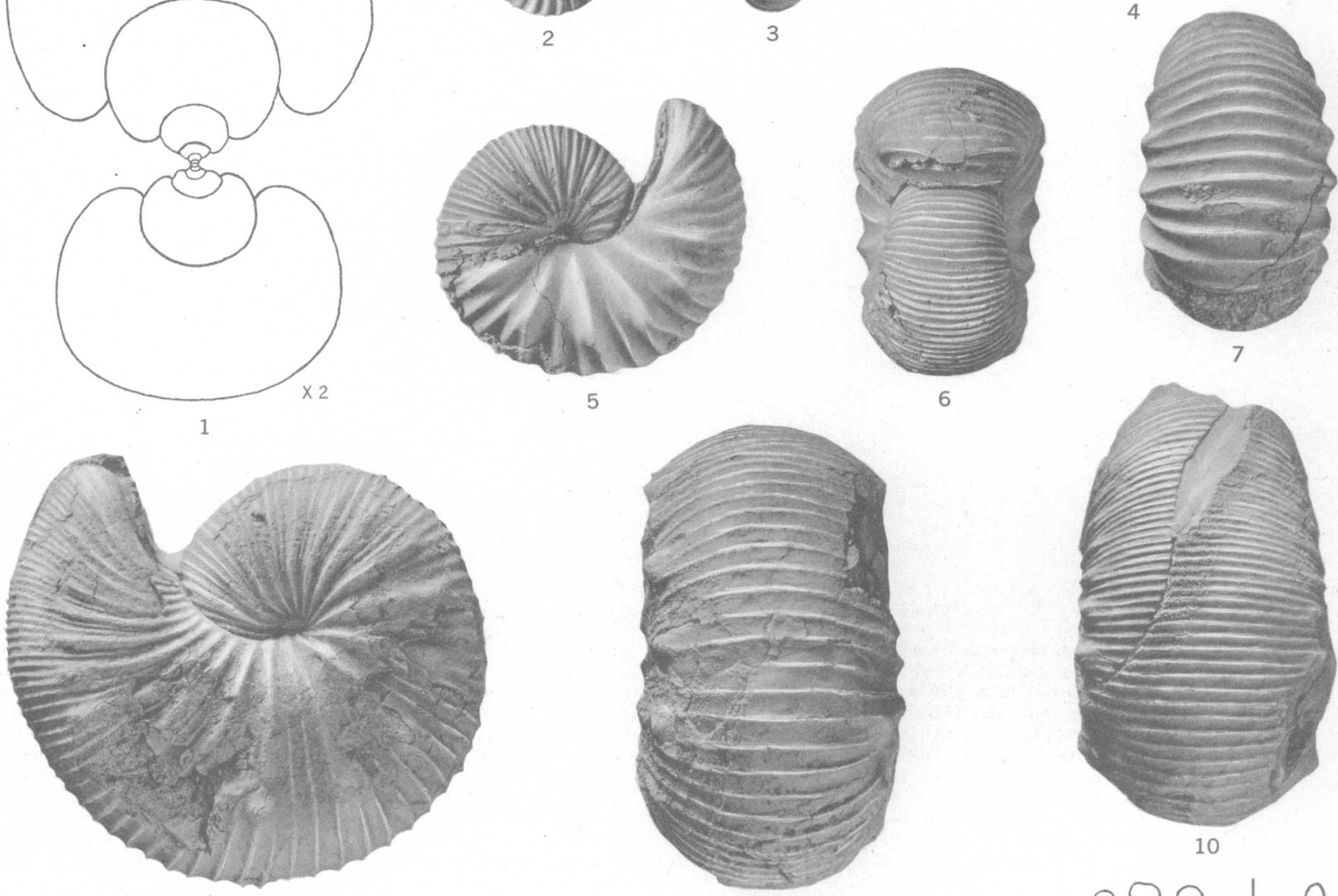

यू|

5
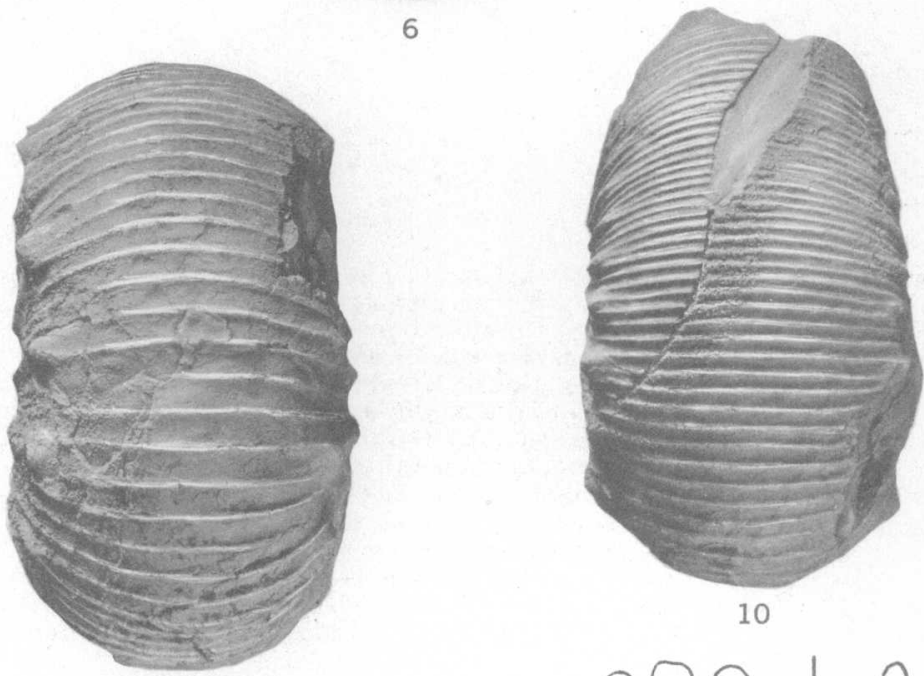

10

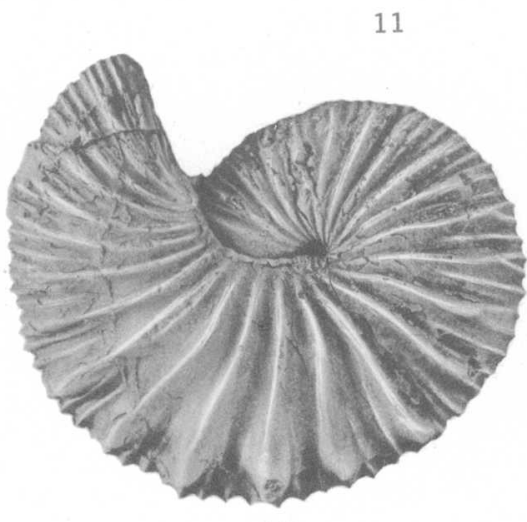

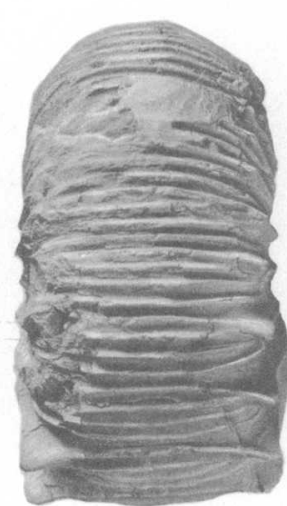

14

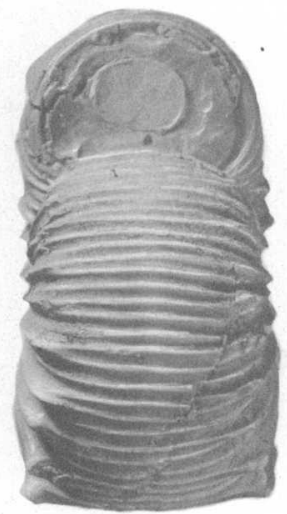

15

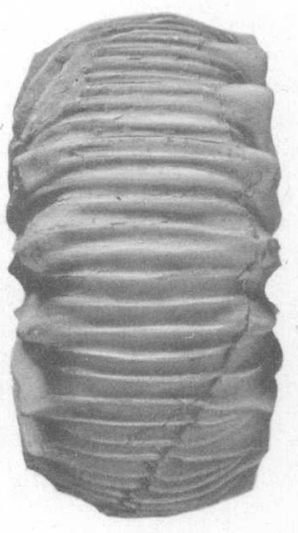

16

SCAPHITES OF THE COLORADO GROUP 


\section{PLATE 21}

[All figures natural size except as indicated on plate]

Figures 1-9. Clioscaphites novimexicanus (Reeside). 1-3, Side and top views, and third from last suture of a nearly complete internal mold with some of the shell, U.S.N.M. 106722a. From near top of Colorado shale at map locality 14. 4-6, Side, front, and rear views of the internal whorls of a specimen at diameter of $16.5 \mathrm{~mm}$., U.S.N.M. $106721 \mathrm{~b}$. From a calcareous concretion 10 feet below top of Colorado shale at map locality 30.7 , Suture of an incomplete specimen, U.S.N.M. 106721a, from same locality as figures 4-6. 8, Side view of an artificial cast made from the impression of a specimen, U.S.N.M. $106722 \mathrm{~b}$, from same locality as figures 1-3. 9, Rear view of a fragment of a stout variant, an internal mold, U.S.N.M. 106723. From a concretionary bed 23 feet below top of Colorado shale at map locality 13 (p. 37)

10-23. Desmoscaphites erdmanni Cobban, n. sp. From same locality as figures 4-6. 10,11, Side and rear views of holotype, an internal mold of most of a living chamber, U.S.N.M. 106724. 12, 13, Side and front views of an incomplete specimen showing the coarse-ribbed outer septate whorl and beginning of the fine-ribbed living chamber, U.S.N.M. 106725c. 14-18, Side and rear views, and last suture of an internal mold of a young specimen with part of the living chamber, U.S.N.M. 106725a. 19, Cross section of septate whorls at diameter of $9 \mathrm{~mm}$. of an internal mold, U.S.N.M. 106725b. 20-23, Side and front views of a septate internal mold showing constrictions and beginning of ribbing, U.S.N.M.'106725d (p. 38).

24-26. Scaphites leei Reeside. From same locality as figures 4-6. 24, 25, Rear and side views of internal mold of a living chamber and part of septate whorl, U.S.N.M. 106720b. 26, Next to last suture of a plesiotype, U.S.N.M. 106720a (p. 34). 

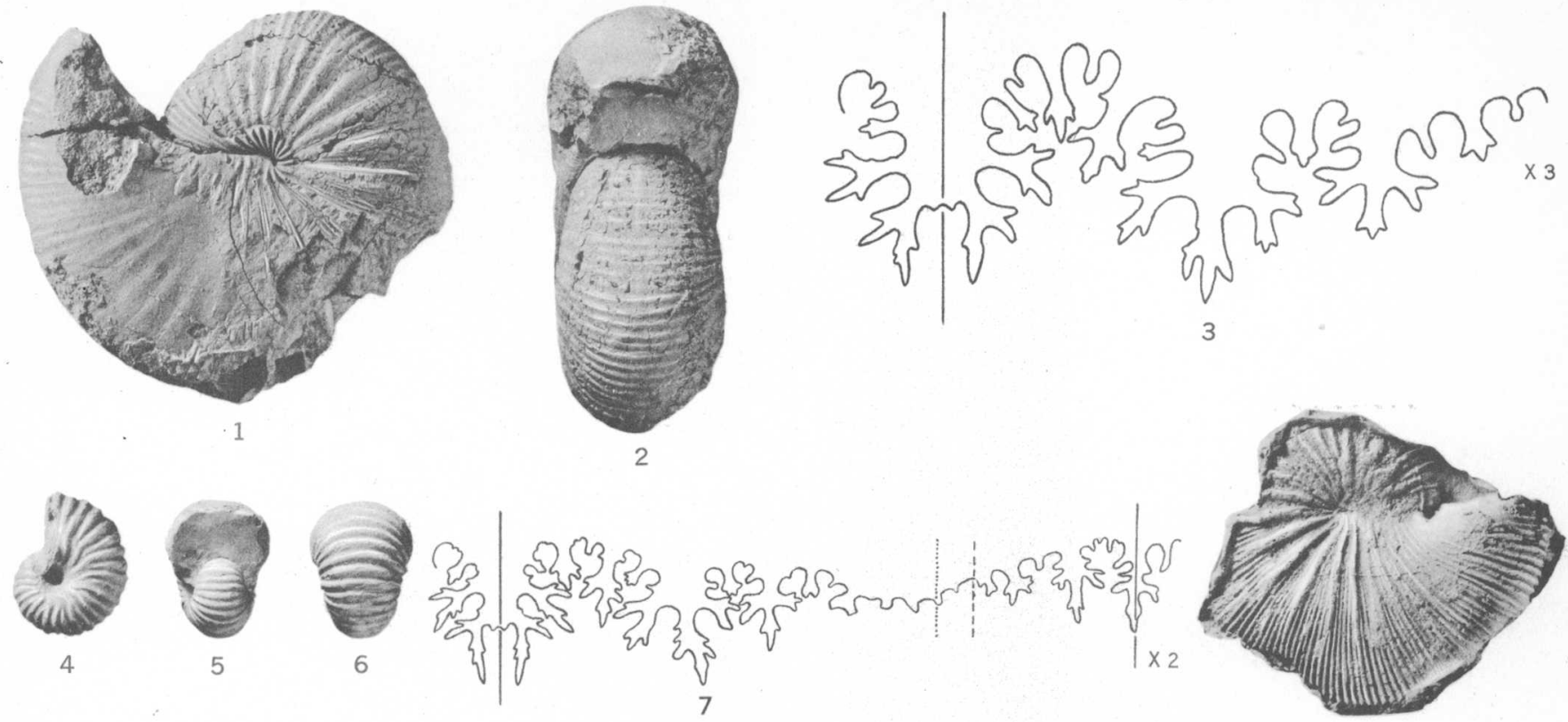

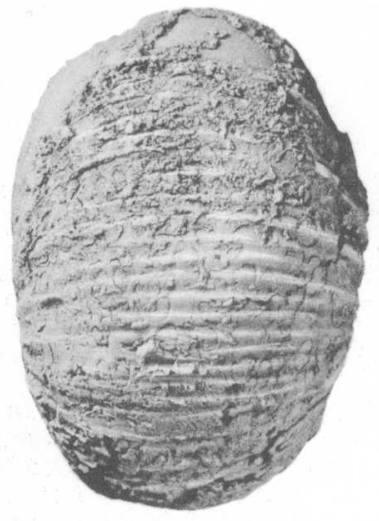

9

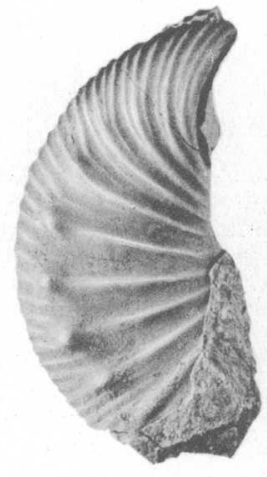

10

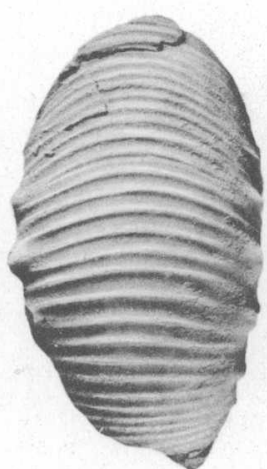

11

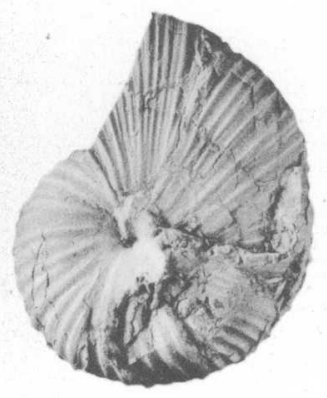

12

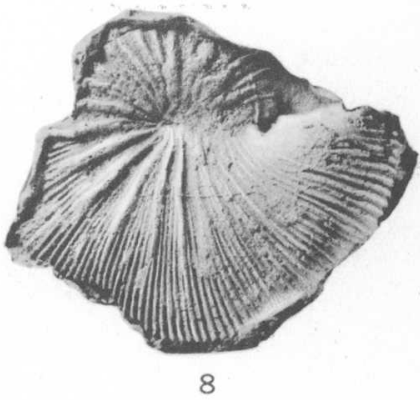

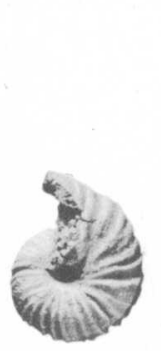

14

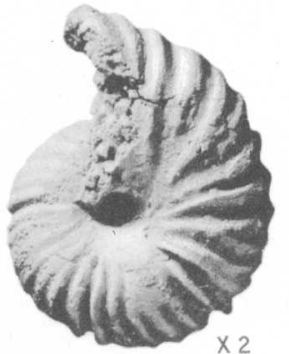

15
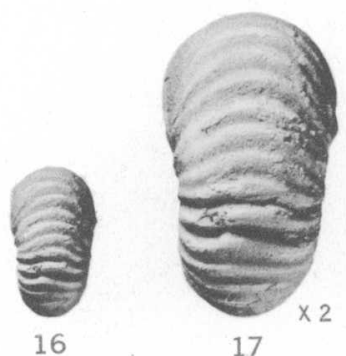

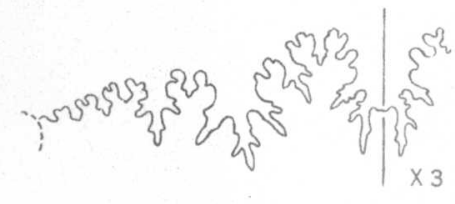

18

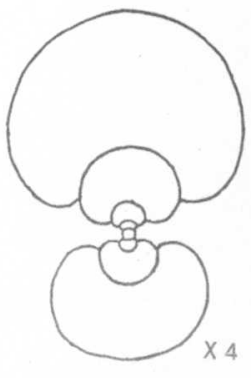

19

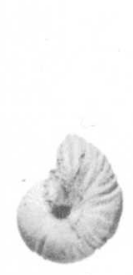

20

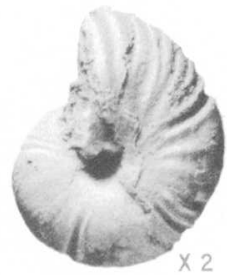

21

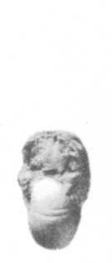

22

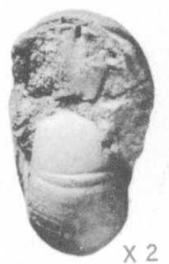

23

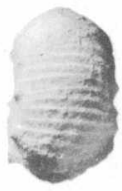

24

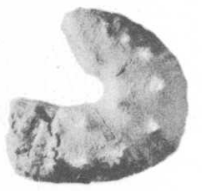

25

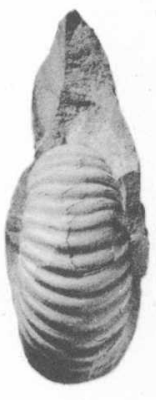

13

SCAPHITES OF THE COLORADO GROUP 